NUREG/CR-2850 PNL-4221 Vol. 4

\title{
Population Dose Commitments Due to Radioactive Releases from Nuclear Power Plant Sites in 1982
}

Prepared by D. A. Baker, R. A. Peloquin

Pacific Northwest Laboratory

Operated by

Battelle Memorial Institute

Prepared for

U.S. Nuclear Regulatory

Commission 
This report was prepared as an account of work sponsored by an agency of the United States Government. Neither the United States Government nor any agency thereof, or any of their employees, makes any warranty, expressed or implied, or assumes any legal liability of re. sponsibility for any third party's use, or the results of such use, of any information, apparatus, product or process disclosed in this report, or represents that its use by such third party would not infringe privately owned rights.

\section{NOTICE}

Availability of Reference Materials Cited in NRC Publications

Most documents cited in NRC publications will be available from one of the following sources:

1. The NRC Public Document Room, 1717 H Street, N.W. Washington, DC 20555

2. The Superintendent of Documents, U.S. Government Printing Office, Post Office Box 37082. Washington, DC 20013-7082

3. The National Technical Information Service, Springfield, VA 22161

Although the listing that follows represents the majority of documents cited in NAC publications, it is not intended to be exhaustive.

Referenced documents available for inspection and copying for a fee from the NRC Public Docu ment Room include NAC correspondence and internal NRC memoranda; NRC Office of Inspection and Enforcement bulletins, circulars, information notices, inspection and investigation notices; Licensee Event Reports; vendor reports and correspondence; Commission papers; and applicant and licensee documents and correspondence.

The following documents in the NUREG series are available for purchase from the GPO Sales Program: formal NAC staff and contractor reports, NRC-sponsored conference proceedings, and NAC booklets and brochures. Also available are Regulatory Guides, NRC regulations in the Code of Federal Regulations, and Nuclear Regulatory Cammission Issuances.

Documents available from the National Technical Information Service include NUREG series reports and technical reports prepared by other federal agencies and reports prepared by the Atomic Energy Commission, forerunner agency to the Nuclear Regulatory Commission.

Documents available from public and special technical libraries include all open literature items, such as books, journal and periodical articles, and transactions. Federal Register notices, federal and state legislation, and congressional reports can usually be obtained from these libraries.

Documents such as theses, dissertations, foreign reports and translations, and non-NRC conference proceedings are available for purchase from the organization sponsoring the publication cited.

Singie copies of NRC draft reports are available free, to the extent of supply, upon written request to the Division of Technical Information and Document Control, U.S. Nuclear Regulatory Com. mission, Washington, DC 20555.

Copies of industry codes and standards used in a substantive manner in the NAC regulatory process are maintained at the NAC Library, 7920 Norfolk Avenue, Bethesda, Maryland, and are available there for reference use by the public. Codes and standards are usually copyrighted and may be purchased from the originating organization or, if they are American National Standards, from the American National Standards Institute, 1430 Broadway, New York, NY 1001 B. 
NUREG/CR-2850

PNL-4221

Vol. 4

\title{
Population Dose Commitments Due to Radioactive Releases from Nuclear Power Plant Sites in 1982
}

Manuscript Completed: April 1986

Date Published: June 1986

Prepared by

D. A. Baker, A. A. Peloquin

Pacific: Northwest Laboratory

Fichland, WA 99352

\author{
Prepared for \\ Division of Budget and Analysis \\ Office of Resource Management \\ U.S. Nuclear Regulatory Commission \\ Washington, D.C. 20555 \\ NRC FIN B2243
}




\section{PREVIOUS REPORTS IN THIS SERIES}

1. Population Dose Commitments Due to Radioactive Releases from Nuclear Power Plant Sites in 1975, PNL-2439, October 1977.

2. Population Dose Comitments Due to Radioactive Releases from Nuclear Power Plant Sites in 1976, NUREG/CR-1125, PNL-2940, December 1979.

3. Population Dose Commitments Due to Radioactive Releases from Nuclear Power Plant Sites in 1977, NUREG/CR-1498, PNL-3324, October 1980.

4. Population Dose Commitments Due to Radioactive Releases from Nuclear Power Plant Sites in 1978, NUREG/CR-2201, PNL-4039, June 1982.

5. Population Dose Commitments Due to Radioactive Releases from Nuclear Power Plant Sites in 1979, NUREG/CR-285D, PNL-4221, Vol. 1, December 1982.

6. Population Dose Commitments Due to Radioactive Releases from Nuclear Power Plant Sites in 1980, NUREG/CR-2850, PNL-4221, Vol. 2, August 1983.

7. Population Dose Commitments Due to Radioactive Releases from Nuclear Power Plant Sites in 1981, NUREG/CR-2850, PNL-4221, Vol. 3, January 1985. 


\section{ABSTRACT}

Population radiation dose commitments have been estimated from reported radionuclide releases from commercial power reactors operating during 1982. Fifty-year dose commitments from a one-year exposure were calculated from both liquid and atmospheric releases for four population groups (infant, child, teen-ager and adult) residing between 2 and $80 \mathrm{~km}$ from each of 51 sites. This report tabulates the results of these calculations, showing the dose commitments for both liquid and airborne pathways for each age group and organ. Also included for each site is a histogram showing the fraction of the total population within 2 to $80 \mathrm{kr}$ around each site receiving various average dose commitments from the airborne pathways.

The total dose commitments from both liquid and airborne pathways ranged from a high of 30 person-rem to a low of 0.007 person-rem for the sites with plants operating throughout the year with an arithmetic mean of 3 person-rem. The total population dose for all sites was estimated at 130 person-rem for the 100 million people considered at risk.

The average individual dose commitment from all pathways on a site basis ranged from a low of $6 \times 10^{-7}$ mrem to a high of $0.06 \mathrm{mrem}$. No attempt was made in this study to determine the maximum dose commitment received by any one individual from the radionuclides released at any of the sites. 



\section{CONTENTS}

ABSTRACT .......................... ACKNOWLEDGMENTS ...................... ix

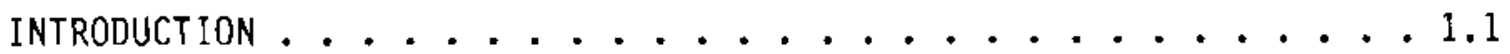

Site-Dependent Parameters .............. 1.4

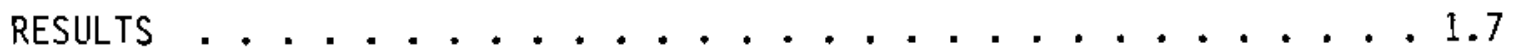

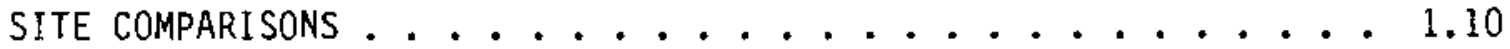

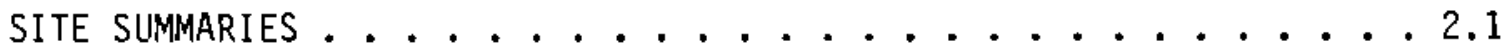

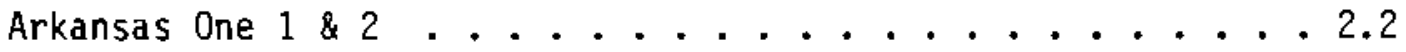

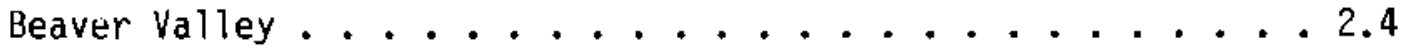

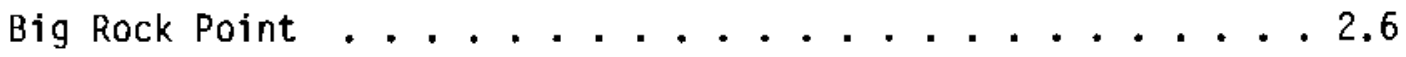
Browns Ferry 1,2, \& $3 \ldots \ldots . \ldots \ldots$ Brunswick $1 \& 2 \ldots \ldots \ldots . \ldots \ldots$ Calvert Cliffs $1 \& 2 \ldots \ldots . \ldots . \ldots . \ldots . \ldots 2$ Cook $1 \& 2 \ldots \ldots \ldots \ldots . \ldots \ldots . \ldots \ldots$

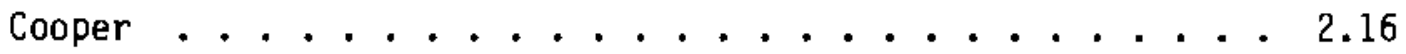

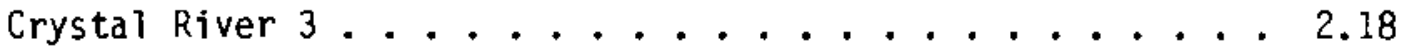
Davis-Besse .................... 2.20 Dresden $1,2, \& 3 \ldots \ldots \ldots . \ldots \ldots . \ldots \ldots$ Duane Arnold ......................... 2.24

J. M. Farley 1 \& $2 \ldots \ldots \ldots . \ldots . \ldots . \ldots 26$

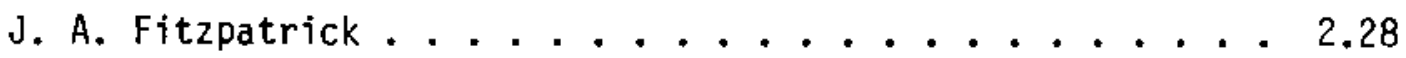




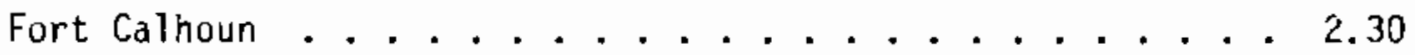

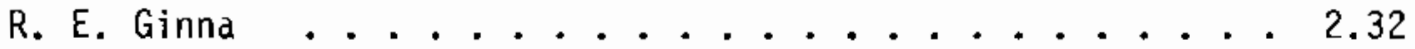

Haddam Neck . . . . . . . . . . . . . 2.34

Edwin I. Hatch $1 \& 2 \ldots \ldots . \ldots \ldots$

Indian Point $1,2, \& 3 \ldots \ldots . \ldots . \ldots . . \ldots$

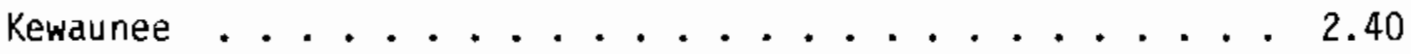

LaCrosse ........................ 2.42

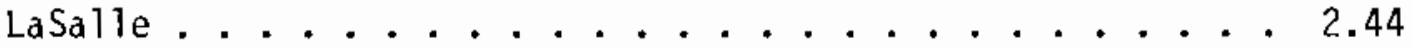

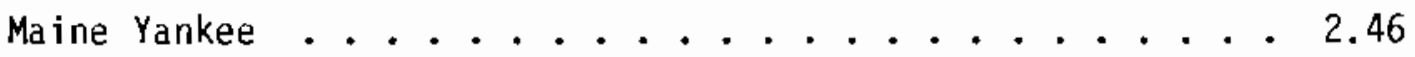

McGuire .................... 2.48

Millstone $1 \& 2 \ldots \ldots \ldots . \ldots \ldots$

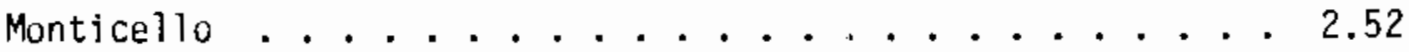

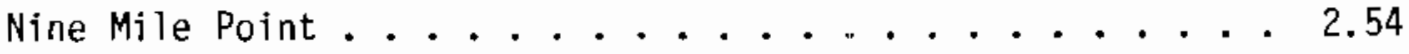

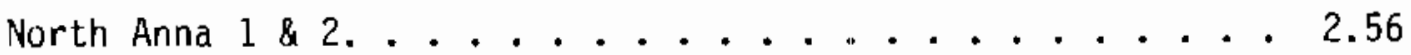

oconee $1,2 \& 3 \ldots \ldots \ldots \ldots \ldots$

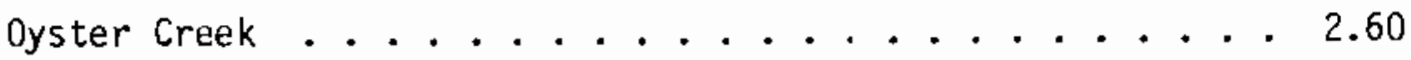

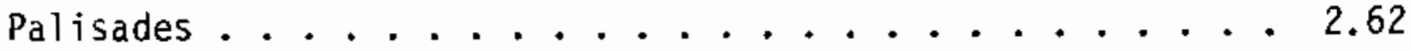

Peach Bottom $2 \& 3 \ldots \ldots . \ldots . \ldots . . \ldots 2 . \ldots$

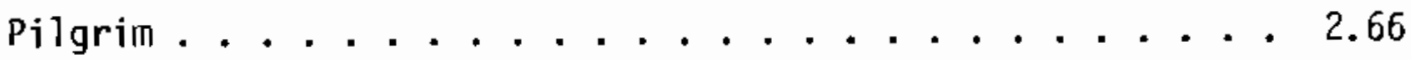

Point Beach $1 \& 2 \ldots \ldots . \ldots \ldots$

Prairie Istand $1 \& 2 \ldots \ldots . \ldots . \ldots 2 . \ldots$

Quad Cities $1 \& 2 \ldots \ldots . \ldots . \ldots . \ldots . \ldots 2$ 
Rancho Seco.................... 2.74

H. B. Robinson ................ 2. 2.76

St. Lucie . . . . . . . . . . . . . . . . 2.78

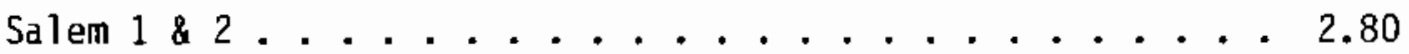

San Onofre 1,2, \&3 ............... 2. 2.82

Sequoyah ...................... 2.84

Summer ................. 2... 2. . . . . . . .

Surry $1 \& 2$ \& . . . . . . . . . . . . . 2.88

Susquehanna .................... 2. . . . . . . . . . 90

Three Mile Island 1 \& 2 ............... 2.92

Trojan ...................... 2.94

Turkey Point $3 \& 4$ \&................. 2. . . . . . . . . .

Vermont Yankee ................. 2. . . . . . . . . .

Yankee Rowe .................... 2. . . . . . 100

Zion 1 \& 2 . . . . . . . . . . . . . . 2. 202

REFERENCES . . . . . . . . . . . . . . . . . 3.1

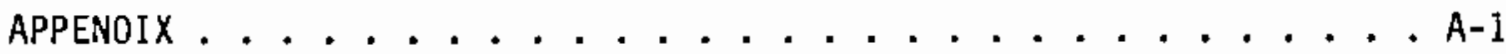





\section{ACKNOWLEDGMENTS}

The authors greatly appreciate the development of the population distributions around each site from census data by Anton A. Sinisgalli, A. R. Brauner, and Donald P. Cleary of the U. S. Nuclear Regulatory Commission. The authors also gratefully acknowledge the contributions and suggestions by Maurice R. Beebe and Charles A. Willis, also of the U.S. NRC. 

INTRODUCTION

Most commercial nuclear power reactors release small amounts of radioactive materials to the environment during normal operation. Because of these releases, concern was expressed about the magnitude of the collective dose received by the general population residing around these nuclear power plants. (a) In response to this concern, the Pacific Northwest Laboratory (PNL) ${ }^{(a)}$ contracted with the Nuclear Regulatory Commission (NRC) to undertake a series of studies to estimate radiation effective dose comnitments produced by radionuclide releases from commercial light-water-cooled power reactors during the years 1975 thru 1981 (see previous reports in this series, p. ii). This document is a continuation of these studies and considers the doses from releases during 1982. In this study, as in thase previous, we estimated the collective (population) dose commitment ${ }^{b}$ from both the liquid and gaseous releases to four age groups making up the population residing in the region of the site: infant ( 0 to $1 \mathrm{yr}$ ), child (1 to $11 \mathrm{yr}$ ), teen-ager (11 to $17 \mathrm{yr}$ ) and adult (17 $\mathrm{yr}$ and older).

The particular organs of reference in this study are listed in Table 1. The major pathways by which radionuclides travel from the reactor to the individual receptors are shown in Table 2. Dther possible liquid pathways such as direct exposure from waterborne activities (swimming, boating, shoreline recreation) and internal exposure through ingestion of food produced using contaminated irrigation water were not included; we have found from past experience that the doses from these pathways are generally much smaller than the doses from the pathways considered in this study.

The "source terms" used to estimate dose commitments produced from each site were the annual measured releases of radioactive materials as reported to the NRC by the plant operators and subsequently published in an NRC public document (Tichler and Norden 1985).

The regional population for which we estimated dose cormitments included those persons estimated to be living in a region between 2 and

(a) Operated by Battelle Memorial Institute for the Department of Energy.

(b) As used in this report, dose commitment describes the total-body dose equivalent in rem ( 1 rem $=0.01$ sievert) received over 50 years from intake during the year in which radioactive materials were released into the environment from the plants. 
TABLE 1. Organs Considered in This Study

Organs Affected by
Airborne Releases

Total body

Thyroid

Bone

GI tract

Liver

Lung
Organs Affected by

Waterborne Releases

Total body

Thyroid

Bone

GI tract

Liver

TABLE 2. Pathways Considered in This Study by Which Radionuclides Travel from Reactors to Persons

Pathways for

Airborne Releases

Air submersion

Contaminated ground

Inhalation

Ingestion of food crops

and animal products
Pathways for Waterborne Releases

Ingestion of drinking water

Ingestion of fish and

invertebrates

$80 \mathrm{~km}$ around the reactor sites during 1982. Population distributions were supplied by the NRC's Office of Nuclear Reactor Regulation.

Atmospheric transport factors (annual average dilution and annual average deposition) were calculated for the region around each site using appropriate meteorological data supplied by the NRC's Office of Nuclear Reactor Regulation. To calculate the doses, we used models approved by the NRC. We incorporated these models into two small computer codes to expedite the dose calculations involved for each site.

Site-specific parameters other than releases, meteorology and population were obtained from environmental statements for the various reactors when available (Table 3). Such parameter values include the total population drinking contaminated water, river flow, dilution flow from the reactors (for sites not on rivers), fish and invertebrate harvest for region, and dilution factors for drinking water and aquatic foods. In those cases in which site-specific data are not readily available and the particular pathway is not expected to result in a large dose, pessimistic assumptions have been used to estimate doses. The use of more realistic data should result in lower dose estimates in some cases. 


\section{TABLE 3. Environmental Statements for Power Plants Included in This Study}

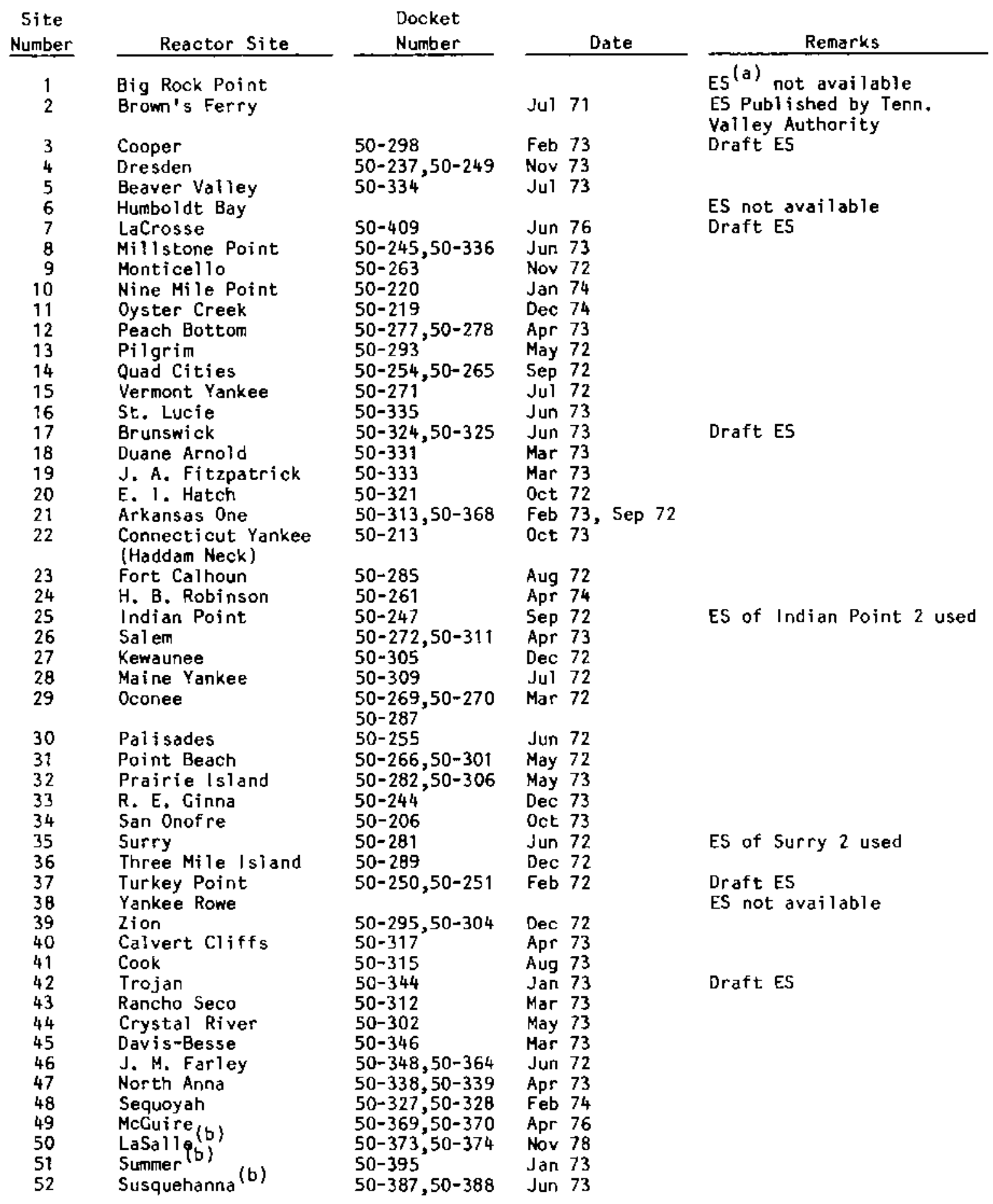

(a) Environmental Statement.

(b) Sites added for 1982 . 
The reactors included in this study, their type, licensed thermal power rating and net electrical output for 1982 are 1 isted in Table 4. Those boiling-water reactors which had an operating augmented gaseous radioactive waste system in 1982 are identified in the table. Populations at risk and the dose comitments derived in the study are also tabulated.

\section{Site-Dependent Parameters}

In the Site Summaries section, the location (including latitude and longitude) for each reactor site and the estimated 1982 population within 2 to $80 \mathrm{~km}$ around each site is given. In addition, the locations of major metropolitan centers within $80 \mathrm{~km}$ are listed along with their 1981 extrapolated populations. The populations of the Standard Metropolitan Statistical Areas (SMSA) are given where applicable. Next, the sitespecific data pertinent to the airborne pathways are specified. The average production rates of vegetable crops and animal products are given for the area within an $80-\mathrm{km}$ radius based upon the statewide average. This production has been reduced for sites on lakes and seacoasts to account for the presence of the body of water. An animal grazing factor is estimated for each site location. This factor accounts for the fraction of the year during which grazing animals such as milk cows and beef cattle graze on fresh pasture in the region around the site. After average production rates are given, the period of record and the percent data recovery of the meteorological data used in calculating diffusion factors are indicated.

Various site-dependent factors associated with the waterborne pathways are presented next. For lake and ocean sites, we used the average dilution of plant effluents for the year 1982 specified by Tichler and Norden (1985). For river sites, the average annual river flow is tabulated. This flow was used in place of a dilution flow from the plant to account for dilutions of liquid releases at the locations of probable intake of drinking water and aquatic food catch. Any exceptions to this scheme have been footnoted. Next is shown the estimated 1982 population utilizing drinking water drawn from supplies containing diluted effluents from the site. These are shown with an estimated dilution factor where applicable. Fish and invertebrate catch data taken from the respective plant environmental statement, when available (see Table 3), are listed next, along with estimated dilution factors for the lake and ocean sites. When site-specific fish and invertebrate catch data were not available, the generic consumption rates were used for the particular site. Sites on salt water were assumed to contribute no dose from drinking water. 


\section{TABLE 4. Reactor Characteristics and Population Total-Body Dose Commitments, 1982}

\begin{tabular}{|c|c|c|c|c|c|c|c|c|c|}
\hline \multirow[b]{2}{*}{ Site } & \multirow[b]{2}{*}{ Unit } & \multirow[b]{2}{*}{ Type. } & \multirow{2}{*}{$\begin{array}{l}\text { Ltcensed } \\
\text { Therma } 1 \\
\text { Power } \\
\text { (MW) } \\
\end{array}$} & \multirow{2}{*}{$\begin{array}{c}\text { Electric } \\
\text { Energy } \\
\text { Ceneration } \\
1982 \\
\left(19 . h^{\prime}\right)(a) \\
\end{array}$} & \multicolumn{3}{|c|}{$\begin{array}{c}\text { Population Dose Comnit tnent } \\
\text { (person-rem) }\end{array}$} & \multirow{2}{*}{$\begin{array}{l}\text { Population } \\
\text { ot Risk }\end{array}$} & \multirow{2}{*}{$\begin{array}{l}\text { Average } \\
\text { Individual } \\
\text { Total-Body } \\
\text { Dose } \\
\text { Comitiment } \\
\text { (mren) } \\
\end{array}$} \\
\hline & & & & & Liquid & $\mathrm{Air}$ & Total & & \\
\hline $\begin{array}{l}\text { Arkansas One } \\
\text { Arkansas One }\end{array}$ & $\begin{array}{l}1 \\
2 \text { TOTAL }\end{array}$ & $\underset{P M R}{P M R}$ & $\begin{array}{l}2568 \\
2815 \\
5383\end{array}$ & $\begin{array}{l}3.72 \\
3.81 \\
7.53\end{array}$ & 4.4 & 0.099 & 4.5 & 1. BES & $2.4 E-2$ \\
\hline Beaver Valley & 1 & PWR & 2652 & 2.69 & 0.022 & 0.014 & 0.036 & $3.6 \mathrm{E} 6$ & $1.0 \mathrm{E}-5$ \\
\hline Big Rock Point ${ }^{(b)}$ & 1 & BWR & 240 & 0.36 & 1.0 & 0.16 & 1.2 & $1.7 \mathrm{ES}$ & $6.9 \mathrm{E}-3$ \\
\hline $\begin{array}{l}\text { Bromns Ferry } \\
\text { Browns ferty }\end{array}$ & $\begin{array}{l}1 \\
2 \\
3 \\
\text { TOTAL }\end{array}$ & $\begin{array}{l}\text { BWR } \\
\text { BWR } \\
\text { BuR }\end{array}$ & $\begin{array}{l}3293 \\
3293 \\
3293 \\
9879\end{array}$ & $\begin{array}{r}7.88 \\
4.55 \\
4.89 \\
17.2\end{array}$ & 3.1 & 31 & 34 & $7.2 E 5$ & $4.7 \mathrm{E}-2$ \\
\hline $\begin{array}{l}\text { Brunswick } \\
\text { Brunswick }\end{array}$ & $\begin{array}{l}1 \\
2 \\
\text { TOTAL }\end{array}$ & $\begin{array}{l}\mathrm{BWR} \\
\mathrm{BW}\end{array}$ & $\begin{array}{l}2436 \\
2436 \\
4872\end{array}$ & $\begin{array}{l}2.92 \\
1.91 \\
4.83\end{array}$ & 0.011 & 14 & 14 & $2.2 \mathrm{ES}$ & $6.4 E-2$ \\
\hline $\begin{array}{l}\text { Calvert Cliffs } \\
\text { Calvert Cliffs }\end{array}$ & $\begin{array}{l}1 \\
2 \text { TOTAL }\end{array}$ & $\underset{P M R}{P M R}$ & $\begin{array}{l}2700 \\
2700 \\
5400\end{array}$ & $\begin{array}{r}5.36 \\
5.00 \\
10.36\end{array}$ & 1.9 & 0.25 & 2.2 & $2.6 \mathrm{E} 6$ & $8.3 E-4$ \\
\hline $\begin{array}{l}\text { Cook } \\
\text { Cook }\end{array}$ & $\begin{array}{l}1 \\
2 \\
\text { TOTAL }\end{array}$ & $\underset{P W R}{P M R}$ & $\begin{array}{l}3250 \\
3391 \\
6641\end{array}$ & $\begin{array}{r}5.35 \\
7.00 \\
12.35\end{array}$ & 0.65 & 0.071 & 0.72 & $1.2 E 6$ & $6.3 \mathrm{E}-\mathrm{h}$ \\
\hline Cooper & 1 & BuR & 2381 & 5.28 & 0.027 & 0.14 & 0.17 & $1.7 \varepsilon 5$ & 1. $8 \mathrm{E}-4$ \\
\hline Crystal River & 3 & $P$ P & 2452 & 4.92 & 0.0058 & 0.025 & 0.031 & $3.7 \mathrm{E5}$ & 8. $2 \mathrm{E}-5$ \\
\hline Davis-Besse & $\uparrow$ & PWR & 2772 & 3.22 & 0.068 & 0.018 & 0.086 & 1.BE6 & 4. $8 \mathrm{E}-5$ \\
\hline Dresden & $\begin{array}{l}1 \\
2 \\
3\end{array}$ & $\begin{array}{l}8 \times R \\
B m \\
B \times R\end{array}$ & $\begin{array}{r}700 \\
2527 \\
2527\end{array}$ & $\begin{array}{r}0 \\
5.12 \\
3.89\end{array}$ & & & & & \\
\hline Dresden & TOTAL & & 5754 & 9.01 & 0 & 6.2 & 6.2 & $6.4 E 6$ & $9.7 \mathrm{E}-4$ \\
\hline Duane Arnold & 1 & $B M$ & 1638 & 2.28 & $9 E-B$ & 0.0076 & 0.0076 & 6.0E5 & $1.3 \mathrm{E}-5$ \\
\hline $\begin{array}{l}\text { J. M. Farley } \\
\text { J. M. Farley }\end{array}$ & $\begin{array}{l}1 \\
2 \text { TOTAL }\end{array}$ & $\begin{array}{l}\text { PWR } \\
\text { PWR }\end{array}$ & $\begin{array}{l}2652 \\
2652 \\
5304\end{array}$ & $\begin{array}{r}5.22 \\
5.3 \mathrm{C} \\
10.52\end{array}$ & 0.043 & 1.5 & 1.5 & $3.6 \mathrm{ES}$ & $4.3 \mathrm{E}+3$ \\
\hline J. A. Fitzpatrick & 1 & BMR & 2436 & 4.96 & 0.13 & 6.8 & 6.9 & 8.4ES & 6. $2 \mathrm{E}-3$ \\
\hline Fort Calhoun & 1 & PWR & 1420 & 3.48 & 0.35 & 0.005 & 0.36 & $7.6 E 5$ & 4. $7 E-4$ \\
\hline R. E. Cinna & $\uparrow$ & PAR & 1520 & 2.41 & 0.18 & 0.051 & 0.23 & $1.2 \mathrm{EG}$ & $1,9 E-4$ \\
\hline Haddam Meck & 1 & PWR & 1825 & 4.54 & 0.033 & 0.17 & 0.20 & $3.4 E 6$ & $5.9 \mathrm{E}-5$ \\
\hline $\begin{array}{l}\text { E. I. Hatch } \\
\text { E. I. Hatch }\end{array}$ & $\begin{array}{ll}1 & \\
2 & \\
& \text { TOTAL }\end{array}$ & $\begin{array}{l}\mathrm{BWh} \\
\mathrm{BWR}\end{array}$ & $\begin{array}{l}2436 \\
2436 \\
4877\end{array}$ & $\begin{array}{l}2.88 \\
3.73 \\
6.61\end{array}$ & 2.8 & 0.042 & 2.8 & $3.0 \mathrm{ES}$ & $9.3 E-3$ \\
\hline $\begin{array}{l}\text { Indian Point } \\
\text { Indian Point }\end{array}$ & $\begin{array}{lr}9 & \\
2 & \\
3 & \\
& \text { TOTAL }\end{array}$ & $\begin{array}{l}P W R \\
P W R \\
P W R\end{array}$ & $\begin{array}{r}615 \\
2758 \\
2760 \\
6133\end{array}$ & $\begin{array}{l}0 \\
4.45 \\
1.49 \\
5.89\end{array}$ & 0.70 & 4.8 & 5.5 & $1.5 E 7$ & $3.6 \mathrm{E}-4$ \\
\hline Kenaunee & 1 & PMP & 1650 & 3.82 & 1.7 & 0.003 & 1.7 & $6.2 \mathrm{E5}$ & $2.8 E-3$ \\
\hline LaCrosse ${ }^{(b)}$ & 1 & BWR & 165 & 0.14 & 6.7 & 0.75 & 7.0 & 3.5E5 & $2,0 \mathrm{E}-2$ \\
\hline LaSalle & 1 & $B M R$ & 3323 & 0.46 & 0 & 0.0004 & 0.0001 & $1.0 E 6$ & $9.6 \mathrm{E}-6$ \\
\hline Maine Yonkee & 1 & PMR & 2440 & 4.52 & 0.003 & 0.0042 & 0.072 & $5.9 E 5$ & $1.2 E-5$ \\
\hline McGuire & 1 & PWR & 3411 & 4.3 & 0.24 & 0.15 & 0.39 & $1.6 \mathrm{E} 6$ & $2,4 E-4$ \\
\hline $\begin{array}{l}\text { Millstone } \\
\text { Millstone }\end{array}$ & ${ }^{1}{ }_{\text {TOTAL }}$ & $\begin{array}{l}\text { BWR } \\
\text { PWR }\end{array}$ & $\begin{array}{l}2011 \\
2560 \\
4571\end{array}$ & $\begin{array}{l}+.08 \\
5.01 \\
9.09\end{array}$ & 0.13 & 1.7 & 1.8 & $2.5 E 6$ & $7.2 \mathrm{E}-4$ \\
\hline Monticello & 1 & $\mathrm{BMR}$ & 1670 & 2.42 & $1 E-6$ & 0.19 & 0.19 & $2.2 \mathrm{E} 6$ & 8. $8 E-5$ \\
\hline Nine Mile Point & 1 & $B M R$ & 1850 & 8.13 & 0.014 & 0.014 & 0.028 & $8.4 E 5$ & $3.3 \mathrm{E}-5$ \\
\hline
\end{tabular}

(a) $1 \mathrm{TW}, \mathrm{hr}=3 . \mathrm{fE} 15$ joules.

(b) Do not have charcoal delay beds in the gaseaus effluent line from air ejector. 
TABLE 4. (contd)

\begin{tabular}{|c|c|c|c|c|c|c|c|c|c|}
\hline \multirow[b]{2}{*}{ S1te } & \multirow[b]{2}{*}{ Unit } & \multirow[b]{2}{*}{ Type } & \multirow{2}{*}{$\begin{array}{l}\text { Licensed } \\
\text { Thermal } \\
\text { Power } \\
\text { (MW) } \\
\end{array}$} & \multirow{2}{*}{ 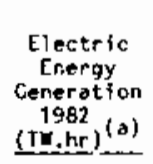 } & \multicolumn{3}{|c|}{$\begin{array}{c}\text { Population Eose Commftment } \\
\text { (persontrem) }\end{array}$} & \multirow{2}{*}{$\begin{array}{l}\text { Population } \\
\text { at Risk }\end{array}$} & \multirow{2}{*}{$\begin{array}{c}\text { Average } \\
\text { Invividual } \\
\text { Total-Body } \\
\text { Dose } \\
\text { Commitment } \\
\text { (mrem) }\end{array}$} \\
\hline & & & & & Lfquid & Air. & Iotal & & \\
\hline $\begin{array}{l}\text { North Anna } \\
\text { North Anna }\end{array}$ & $\begin{array}{l}1 \\
2 \\
\\
\text { TOTAL }\end{array}$ & $\begin{array}{l}\text { PWR } \\
P_{W R R}\end{array}$ & $\begin{array}{l}2775 \\
2775 \\
5550\end{array}$ & $\begin{array}{l}2.40 \\
4.05 \\
6.45\end{array}$ & 5.2 & 0.040 & 5.2 & $1.0 \mathrm{E} 6$ & $5.9 E-3$ \\
\hline $\begin{array}{l}\text { Oconee } \\
\text { Oconee }\end{array}$ & $\begin{array}{ll}1 & \\
2 & \\
3 & \\
& \text { TOTAL }\end{array}$ & $\begin{array}{l}\text { PHR } \\
\text { PWR } \\
\text { PWR }\end{array}$ & $\begin{array}{l}2568 \\
2568 \\
2568 \\
7704\end{array}$ & $\begin{array}{r}5.15 \\
3.44 \\
2.12 \\
10.71\end{array}$ & 9.4 & 0.50 & 9.9 & $9.0 E 5$ & $1.1 \mathrm{E}-2$ \\
\hline Oyster Creek & 1 & $B W R$ & 1930 & 2.01 & 0.011 & 7.6 & 7.6 & $3.5 \mathrm{E} 6$ & $2.2 \mathrm{E}-3$ \\
\hline Palisades & 1 & PWR & 2530 & 3.35 & 0.55 & 0.071 & 0.62 & $1.0 \mathrm{E} 6$ & $5.9 E-4$ \\
\hline $\begin{array}{l}\text { Peach Bottom } \\
\text { Prach Bottom }\end{array}$ & $\begin{array}{l}2 \\
3 \\
\text { TOTAL }\end{array}$ & $\begin{array}{l}\text { Bur } \\
\text { Gire }\end{array}$ & $\begin{array}{l}3293 \\
3293 \\
6586\end{array}$ & $\begin{array}{r}4.79 \\
8.53 \\
13.32\end{array}$ & 3.1 & 2.2 & 5.3 & $4.3 \mathrm{EG}$ & $1.2 E-3$ \\
\hline pilgrim & 1 & BWR & 1998 & 3.29 & 0.032 & 2.3 & 2.3 & $4.3 E 6$ & $5.4 E-4$ \\
\hline $\begin{array}{l}\text { Point Beach } \\
\text { Point. Beach }\end{array}$ & ${ }^{1}$ TOTAL & $\begin{array}{l}\text { PWR } \\
\text { PWR }\end{array}$ & $\begin{array}{l}1518 \\
1518 \\
3036\end{array}$ & $\begin{array}{l}2.70 \\
3.61 \\
6.31\end{array}$ & 0.15 & 0.26 & 0.41 & $6.2 \mathrm{ES}$ & $6.6 \mathrm{E}-4$ \\
\hline $\begin{array}{l}\text { Prairie Island } \\
\text { Pratrie lsland }\end{array}$ & $\begin{array}{l}1 \\
2 \\
\\
\text { TOTAL }\end{array}$ & $\begin{array}{l}\mathrm{PWR} \\
\mathrm{PWRR}\end{array}$ & $\begin{array}{l}1650 \\
1650 \\
3300\end{array}$ & $\begin{array}{l}3.92 \\
3.86 \\
7.78\end{array}$ & 0.003 & 0.064 & 0.067 & $2.2 \mathrm{E} 6$ & $3.0 \mathrm{E}-5$ \\
\hline $\begin{array}{l}\text { Quad Cities } \\
\text { Quad Cities }\end{array}$ & $i^{i}$ TOTAL & $\begin{array}{l}\text { BuR } \\
\text { BWR }\end{array}$ & $\begin{array}{l}2511 \\
7511 \\
5022\end{array}$ & $\begin{array}{l}3.24 \\
5.06 \\
8.30\end{array}$ & 0.20 & 0.84 & 1.0 & $7.2 \mathrm{ES}$ & $1.4 \mathrm{E}-3$ \\
\hline Rancho Seco & $\uparrow$ & $P M R$ & 2772 & 3.37 & 1.6 & 0.084 & 1.7 & $1.8 \mathrm{E} 6$ & $9.3 \mathrm{E}-4$ \\
\hline H. B. Robinson & 2 & PWR & 2200 & 2.25 & 0.043 & 0.0037 & 0.047 & $6.7 \mathrm{E} 5$ & $7.0 \mathrm{E}-5$ \\
\hline St. Lucie & 1 & $P M R$ & 2560 & 6.78 & 0.12 & 0.75 & 0.87 & $5.8 E 5$ & $1.5 \mathrm{E}-3$ \\
\hline $\begin{array}{l}\text { Salem } \\
\text { Salem }\end{array}$ & $\begin{array}{l}1 \\
2 \\
\\
\text { TOTAL }\end{array}$ & $\begin{array}{l}\text { PWR } \\
\text { PWR }\end{array}$ & $\begin{array}{l}3338 \\
3338 \\
6676\end{array}$ & $\begin{array}{r}4.09 \\
7.94 \\
12.03\end{array}$ & 0.28 & 0.30 & 0.58 & $4.7 \mathrm{E} 6$ & $1.2 E-4$ \\
\hline San Onofie & $\begin{array}{l}1 \\
2 \\
3\end{array}$ & $\begin{array}{l}P W R \\
P W W_{R} \\
P W_{R}\end{array}$ & $\begin{array}{l}1347 \\
3410 \\
3390\end{array}$ & $\begin{array}{l}0.51 \\
0.13\end{array}$ & & & & & \\
\hline San Onofre & TOTAL & & & 0.64 & 0.48 & 0.033 & 0.51 & $4.8 E 6$ & $1 . t E-4$ \\
\hline Sequoyah & 1 & PWR & 2815 & 4.91 & 0.80 & 0.45 & 1.2 & 8.4ES & $1.5 E-3$ \\
\hline Summer & 1 & PNR & 2775 & 0.19 & 0.0003 & 0.0002 & 0.0005 & $8.2 \mathrm{ES}$ & $6.1 E-7$ \\
\hline $\begin{array}{l}\text { Surry } \\
\text { surry }\end{array}$ & $\begin{array}{l}1 \\
2 \\
\text { TOTAL }\end{array}$ & $\begin{array}{l}P_{W R} \\
P_{W R}\end{array}$ & $\begin{array}{l}2441 \\
2441 \\
4882\end{array}$ & $\begin{array}{r}5.48 \\
5.49 \\
10.97\end{array}$ & 1.4 & 0.86 & 2.3 & $1.7 \mathrm{E} 6$ & $1.3 E-3$ \\
\hline Susquehanna & 1 & BWR & 3293 & 0.31 & 0.15 & 0.056 & 0.21 & $1.5 \mathrm{E} 6$ & $1.4 E-4$ \\
\hline $\begin{array}{l}\text { Three Mile Island } \\
\text { Three Mile island }\end{array}$ & $i^{1}$ TOTAL & $\begin{array}{l}\text { PWR } \\
P M R\end{array}$ & $\begin{array}{l}2535 \\
2772 \\
5307\end{array}$ & $\begin{array}{l}0 \\
0 \\
0\end{array}$ & 0.087 & 0.19 & 0.28 & $2.1 \mathrm{E} 6$ & $1.3 \mathrm{E}-4$ \\
\hline Trojan & 1 & PWR & 3411 & 4,80 & 0.07 & 0.10 & 0.17 & $1.4 \mathrm{E} 6$ & $1.2 \mathrm{E}-4$ \\
\hline $\begin{array}{l}\text { Turkey Point } \\
\text { Turkey Point }\end{array}$ & $3^{3}$ TOTAL & $\begin{array}{l}\text { PWR } \\
\text { PWR }\end{array}$ & $\begin{array}{l}2200 \\
2200 \\
4400\end{array}$ & $\begin{array}{l}3.77 \\
3.84 \\
7.61\end{array}$ & 0.0051 & 0.30 & 0.31 & $2.4 \mathrm{E} 6$ & $1.3 \mathrm{E}-4$ \\
\hline Vermont Yankee & 1 & $\mathrm{BmR}$ & 1593 & 4.17 & 0 & 0.056 & 0.056 & $1.3 \mathrm{E} 6$ & $4,2 \mathrm{E}=5$ \\
\hline Yankee Rowe & 1 & PWR & 600 & 0.88 & 0.13 & 0.058 & 0.19 & 1.656 & $1.2 E-4$ \\
\hline $\begin{array}{l}\text { Zion } \\
\text { Zion }\end{array}$ & ${ }^{1}$ TOTAL. & $\begin{array}{l}P W_{R} \\
P R_{R}\end{array}$ & $\begin{array}{l}3250 \\
3250 \\
6500\end{array}$ & $\begin{array}{r}4.70 \\
5.16 \\
9.16 \\
\end{array}$ & 0.42 & 2.2 & 2.6 & $7.2 \mathrm{E} 6$ & $\underline{3.7 E^{-4}}$ \\
\hline TOTAL FOR ALL SITES & & & & 274 & 47 & 87 & 130 & $1.0 \mathrm{E} B$ & -- \\
\hline Arithmetic Meon & & & & 5.4 & 0.92 & 1.7 & 2.6 & $2.0 \mathrm{E} 6$ & $4.3 \mathrm{E}-3$ \\
\hline Gecmetric Mean & & & & 1.4 & 0.099 & 0.14 & 0.47 & $1.2 \mathrm{E} 6$ & $3.8 E-4$ \\
\hline
\end{tabular}

(a) 1TM.hr = 3.6E15 joules. 
RESULTS

This report consists of a sumary of values used for site-specific parameters at each site, as explained above, and the results of population dose comitment calculations. The population dose comituents are presented in two tables facing the page summarizing site-specific parameters for that site. These tables include both liquid and aitrome pathway dose comitwents for the several organs of reference for each age group investigated. They also include the dose to the whole population, which includes all age gropps. The airborne population dose comitments for each of 160 segments partitioning the region around the site were divided by the population residing within that segment to derive an average individual dose for that segment. These doses are sumarized as a histogram showing percent of the population receiving a given dose level for each site. The fractional population dose from the liquid pathway was not determined in this manner, because the NRC does not at present take into account the location of individuals exposed via this pathway, except those exposed through ingestion of drinking water.

Population dose commitments estimated for both the liquid pathways and airborne pathways varied widely over the 51 sites (75 reactors) studied. The total dose commitments (from both pathways) varied from a high of 30 to a low of 0.007 person-rem for plants in operation during the whole year. The arithmetic mean for the dose from liquid pathways was 1 person-rem and the mean for the dose from airborne pathways was 2 person-rem (see Table 4).

As in past years, most of the plants accounted for less than a person-rem to their surrounding population from plant releases of radionuclides into liquid effluent streams. The highest population doses from liquid releases were all under 10 person rem (Oconee, 9 person-rem). This dose was due almost totally to radioactive cesium.

The doses from radionuclide releases from plant vents and/or stacks into the atmosphere accounted for less than 1 person-rem for most sites. Only two sites had airborne releases resulting in more than 10 person-rem. Those sites were Browns Ferry (34 person-rem) and Brunswick (14 person-rem). Most of these doses were the result of krypton-88 and its daughters.

(a) See Appendix for definition of segments. 
The total population dose comitments from all sites for 1982 were estimated to be 50 person-rem via liquid pathways and 90 person-rem via airborne pathways (Table 4 ).

Figure 1 is a histogram for all 51 sites taken together. We can see from this plot that about $30 \%$ of the total population at risk (100 million) would each receive a dose commitment of between $1 \times 10^{-4}$ and $3 \times 10^{-4}$ mrem. We can see further that about $2 \%$ receive a dose which is less than $1 \times 10^{-6}$ mrem. Although not discernible from the plot, $0.15 \%$ received a dose of between 0.1 and $10 \mathrm{mrem}$. However, no attempt was made in this study to estimate the maximum dose comitment received by any one individual from the radionuclides released at any of the sites.

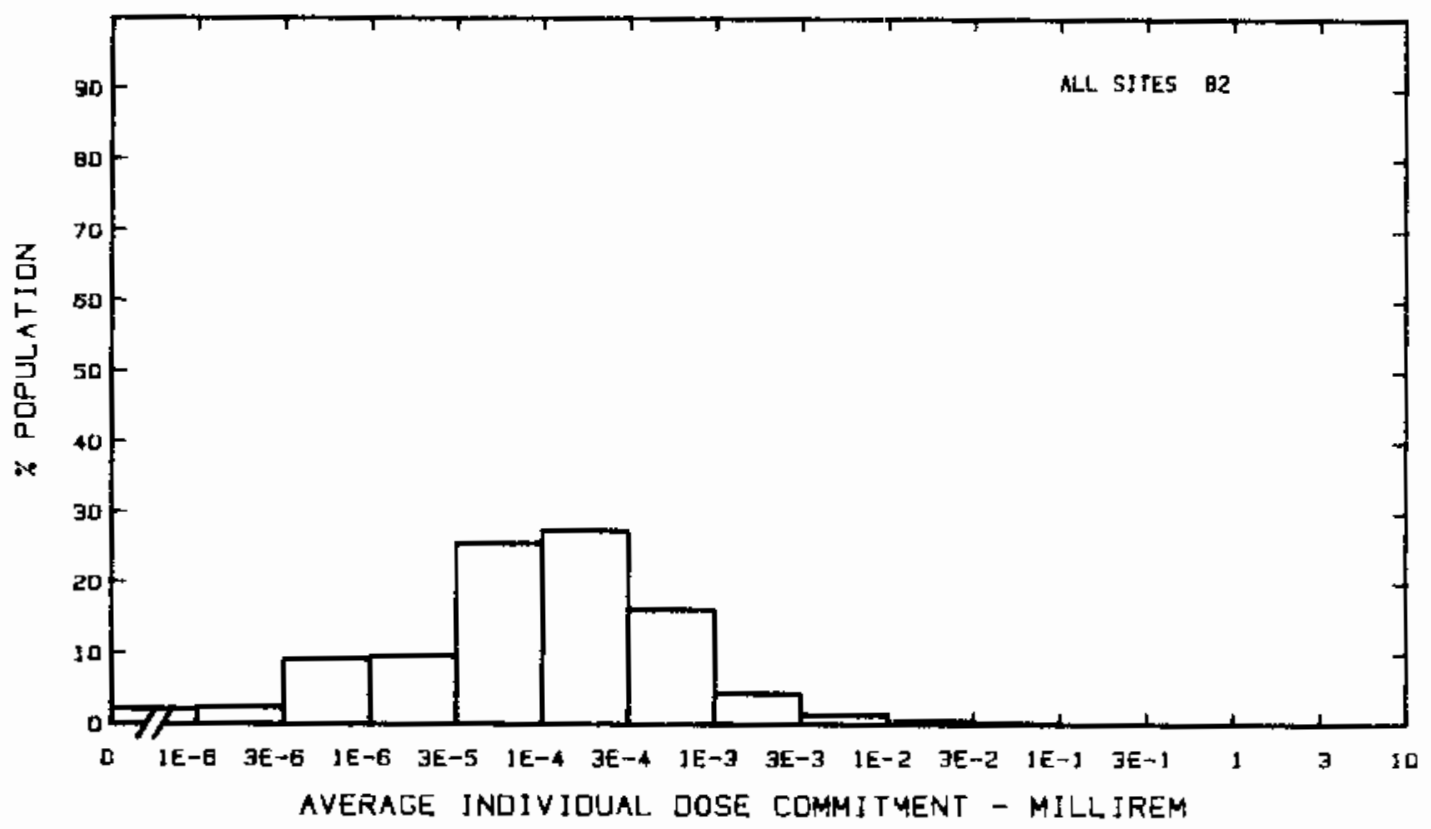

FIGURE 1. Fraction of Total Population Receiving Various Average Individual Total-Body Dose Commitments for All Sites

Figure 2 shows graphically in "Box and Whisker Plots" the wide range of the airborne population dose conmitments for the reactor sites. The median, upper and lower quartiles and upper and lower octiles for the distribution of doses calculated for each of 160 segments are indicated for each site. The upper and lower octiles are shown as the horizontal bars on the "whiskers;" the upper and lower quantiles are indicated as the top and bottom of the "box." The median is the horizontal line inside the box. 


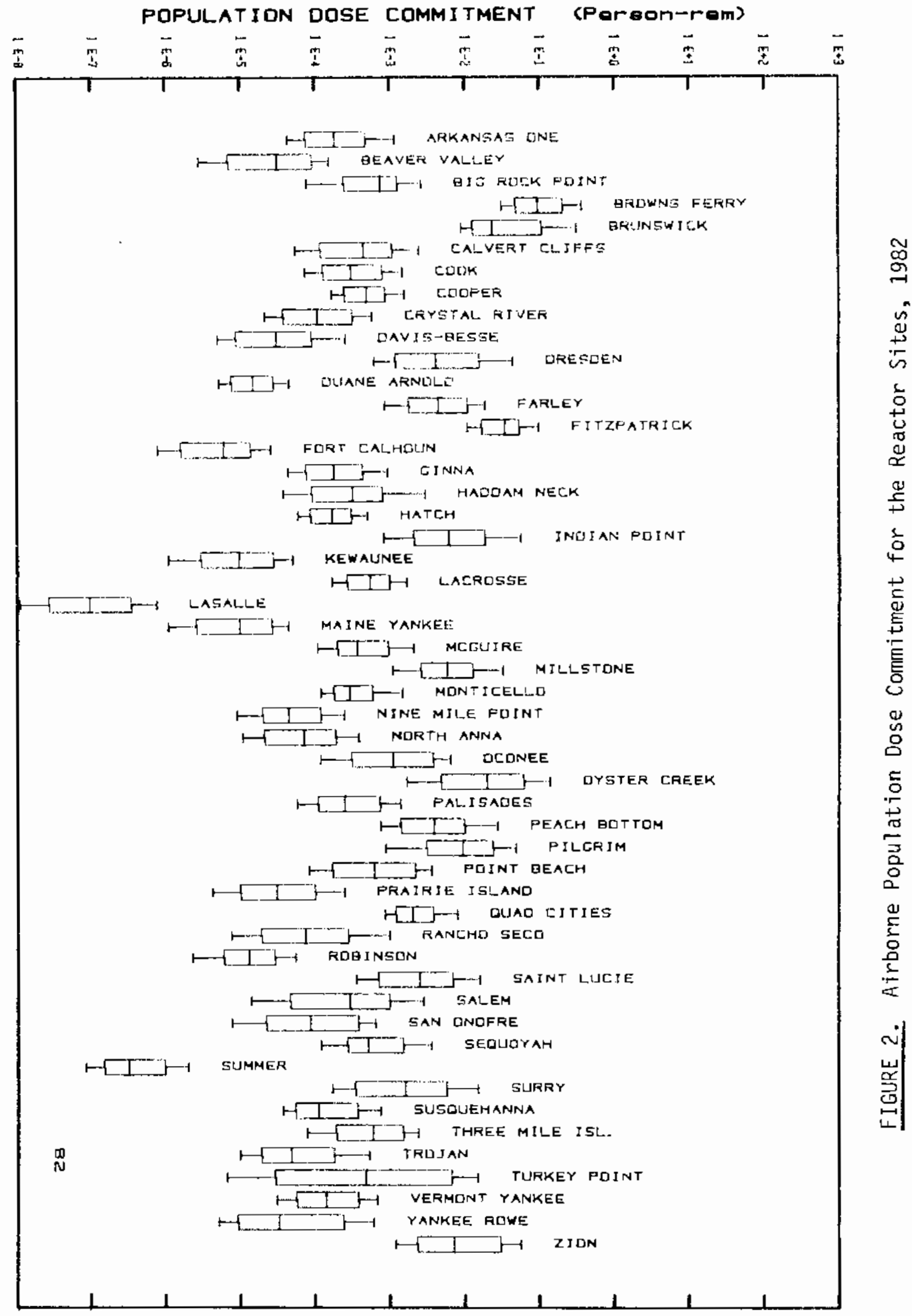


We should point out here, however, that the doses estimated in this study are extremely low compared to an average anriual background dose of approximately $100 \mathrm{mrem}$. We have compared dose comitments calculated in this study with annual background. However, this comparison is not quite exact, since these dose commitments are those total-body doses received from the year's (1982) effluent release -- over 50 years of a person's lifetime. However, most of the dose commitment calculated here is delivered in the first year, so the comparison is reasonably valid.

For comparison purposes, the doses in the site summary tables are given to two significant figures; however, the data and models used to calculate the doses limit their accuracy to at most one significant figure. 


\section{SITE COMPARISONS}

Compared to 1981, the "liquid dose" is lower (47 vs. 93), whereas, the "air dose" is somewhat higher ( 87 vs. 63). Table 5 compares the total population dose commitments estimated for the past 8 years.

TABLE 5. Comparison of Annual Population Dose Commitments for the Last Eight Years (person-rem)

$$
\underline{1975} \quad \underline{1976} \quad \underline{1977} \quad \underline{1978} \quad \underline{1979} \quad \underline{1980} \quad \underline{1981} \quad \underline{1982}
$$

$\begin{array}{lrrrrrrrr}\text { Liquid } & 76 & 82 & 160 & 110 & 220 & 120 & 93 & 47 \\ \text { Air } & \frac{1300}{1300} & \frac{390}{470} & \frac{540}{700} & \frac{530}{640} & \frac{1600}{1800} & \frac{57}{180} & \frac{63}{160} & \frac{87}{130}\end{array}$

The reactor sites were compared as to the total population dose over the years of this study, 1975 - 1982. The sites were placed within six groupings depending on resulting population dose sumuned over each of the years thru 1982:

$\begin{array}{lcc}\text { I } & \text { Greater than } 100 \text { person-rem } \\ \text { II } & 26-100 & " \\ \text { II } & 11-25 & " \\ \text { IV } & 2.6-10 & " \\ \text { V } & 1-2.5 & " \\ \text { VI } \text { Less than 1 } & "\end{array}$

Table 6 shows the sites within the groups along with the reactor manufacturer, year of cormercial operation commencement, and the indicated population doses in person-rem. The manufacturer codes are as follows:

$\begin{array}{ll}\text { AC } & \text { Allis Chalmers } \\ B & \text { Babcox and Wilcox } \\ \text { CE } & \text { Combustion Engineering } \\ \text { GE } & \text { General Electric } \\ \text { W } & \text { Westinghouse }\end{array}$




\section{TABLE 6. Total-Body Population Doses from Nuclear Power Plant Effluents During Norma 1 Operations, ${ }^{\text {a }}$ 1975-1982}

\begin{tabular}{|c|c|c|c|c|c|c|c|c|c|c|}
\hline \multirow{2}{*}{ Site } & \multirow{2}{*}{$\begin{array}{c}\text { Year } \\
\text { Commercial } \\
\text { Operation }\end{array}$} & \multicolumn{8}{|c|}{ Total Person-rem for year } & \multirow[b]{2}{*}{ TOTAL } \\
\hline & & 1975 & 1976 & 1977 & $19: 8$ & 1979 & 1980 & 1981 & 1982 & \\
\hline \multicolumn{11}{|l|}{ 1. (>100 Person-rem) } \\
\hline $\begin{array}{l}\text { Millstone, GE, CE } \\
\text { Dresden, GE } \\
\text { Oyster Creek, GE } \\
\text { Nine Mile Polnt, CE } \\
\text { Oconee, B } \\
\text { Quad Cities, GE }\end{array}$ & $\begin{array}{l}70,75 \\
60,70,71 \\
69 \\
69 \\
73,74,74 \\
72,72\end{array}$ & $\begin{array}{l}750 . \\
360 . \\
47 . \\
69 . \\
9.2 \\
25 .\end{array}$ & $\begin{array}{c}160 . \\
120 . \\
37 . \\
8.7 \\
20 . \\
11 .\end{array}$ & $\begin{array}{c}220 . \\
180 . \\
41 . \\
3.1 \\
38 . \\
4.0\end{array}$ & $\begin{array}{l}200 . \\
170 . \\
110 . \\
0.07 \\
12 . \\
7.30\end{array}$ & $\begin{array}{r}1.8 \\
15 . \\
220 . \\
140 . \\
5.8 \\
6.5\end{array}$ & $\begin{array}{l}2.9 \\
13 . \\
9.5 \\
0.04 \\
10 . \\
42 .\end{array}$ & $\begin{array}{l}4.8 \\
10 . \\
15 . \\
5.2 \\
13 . \\
16 .\end{array}$ & $\begin{array}{l}1.8 \\
6.2 \\
7.6 \\
0.03 \\
9.9 \\
1.0\end{array}$ & $\begin{array}{l}1300 . \\
870 . \\
490 . \\
226 . \\
118 . \\
113 .\end{array}$ \\
\hline \multicolumn{11}{|c|}{ 11. (26- 100 Person-rem $)$} \\
\hline $\begin{array}{l}\text { Zion, } \\
\text { Pilgrim, GE } \\
\text { Peach Bottom, GE } \\
\text { Cook, W } \\
\text { Browns Ferry, GE } \\
\text { Indian Pt., B, W, W } \\
\text { La Crosse, AC } \\
\text { Big Rock Point, CE } \\
\text { Hatch, CE } \\
\text { Brunswick, GE } \\
\text { Davis-Besse, B }\end{array}$ & $\begin{array}{l}73,73 \\
72 \\
74,74 \\
75,78 \\
74,75,77 \\
62,74,76 \\
69 \\
62 \\
75,80 \\
75,77 \\
77\end{array}$ & $\begin{array}{l}6.1 \\
6.2 \\
2.4 \\
0.21 \\
2.9 \\
3.8 \\
7.0 \\
4.6 \\
-.-02 \\
0.02\end{array}$ & $\begin{array}{l}17 . \\
14 . \\
17 . \\
5.0 \\
1.1 \\
9.1 \\
12 . \\
7.7 \\
0.03 \\
0.53 \\
.-\end{array}$ & $\begin{array}{c}22 . \\
52 . \\
11 . \\
23 . \\
3.2 \\
13 . \\
9.4 \\
2.6 \\
35 . \\
6.4 \\
14 .\end{array}$ & $\begin{array}{c}23 . \\
7.3 \\
15 . \\
40 . \\
2.2 \\
8.4 \\
5.9 \\
2.6 \\
2.5 \\
2.5 \\
0.6\end{array}$ & $\begin{array}{l}14 . \\
3.1 \\
30 . \\
17 . \\
9.6 \\
5.9 \\
4.1 \\
9.0 \\
--. \\
2.2 \\
0.52\end{array}$ & $\begin{array}{c}11 . \\
4.3 \\
4.7 \\
0.34 \\
2.8 \\
4.9 \\
2.1 \\
10 . \\
0.34 \\
2.0 \\
11 .\end{array}$ & $\begin{array}{l}1.7 \\
0.74 \\
2.7 \\
0.47 \\
7.8 \\
8.6 \\
4.9 \\
3.6 \\
2.2 \\
1.5 \\
0.65\end{array}$ & $\begin{array}{c}2.6 \\
2.3 \\
5.3 \\
0.72 \\
34 . \\
5.5 \\
6.9 \\
1.2 \\
2.8 \\
14 . \\
0.09\end{array}$ & $\begin{array}{l}97 . \\
90 . \\
88 . \\
87 . \\
64 . \\
59 . \\
52 . \\
41 . \\
40 . \\
29 . \\
27 .\end{array}$ \\
\hline \multicolumn{11}{|c|}{ 111. (11 - 25 Person-rem) } \\
\hline $\begin{array}{l}\text { Kewaunee, W } \\
\text { Haddam Neck, W } \\
\text { Humboldt Bay, GE } \\
\text { Arkansas One, B } \\
\text { Crystal River, B } \\
\text { Surry, } \\
\text { Fitzpatrick, GE } \\
\text { Rancho Seco, B } \\
\text { Horth Anra, W } \\
\text { Calvert Cl iff , CE } \\
\text { Robinson, w } \\
\text { Turkey Point, W }\end{array}$ & $\begin{array}{l}74 \\
68 \\
63 \\
74,79 \\
77 \\
72,73 \\
75 \\
75 \\
77,80 \\
75,77 \\
71 \\
72,73\end{array}$ & $\begin{array}{c}8.5 \\
0.54 \\
18 . \\
0.38 \\
.- \\
5.4 \\
0.09 \\
0.05 \\
-- \\
0.50 \\
9.30 \\
0.22\end{array}$ & $\begin{array}{l}5.1 \\
3.7 \\
5.8 \\
4.5 \\
3.4 \\
1.4 \\
0.01 \\
- \\
0.74 \\
0.28 \\
0.28\end{array}$ & $\begin{array}{l}1.9 \\
2.4 \\
-.- \\
1.6 \\
0.02 \\
3.7 \\
0.56 \\
0.06 \\
-. \\
1.9 \\
0.47 \\
0.40\end{array}$ & $\begin{array}{l}0.60 \\
5.6 \\
-. .7 \\
2.2 \\
0.29 \\
1.4 \\
0.37 \\
0.45 \\
0.48 \\
2.6 \\
0.45 \\
9.4\end{array}$ & $\begin{array}{l}1.8 \\
3.6 \\
-. \\
0.47 \\
0.65 \\
1.0 \\
0.24 \\
0.32 \\
4.9 \\
3.0 \\
0.19 \\
0.17\end{array}$ & $\begin{array}{c}3.0 \\
7.5 \\
-2 . \\
3.4 \\
0.32 \\
1.2 \\
2.8 \\
15 . \\
3.1 \\
1.7 \\
0.2 \\
0.08\end{array}$ & $\begin{array}{c}1.6 \\
0.55 \\
2.0 \\
3.6 \\
19 . \\
1.7 \\
6.7 \\
1.8 \\
4.2 \\
0.72 \\
1.3 \\
0.07\end{array}$ & $\begin{array}{l}1.7 \\
0.20 \\
-. \\
4.5 \\
0.03 \\
2.3 \\
6.9 \\
1.7 \\
5.2 \\
2.2 \\
0.05 \\
0.31\end{array}$ & $\begin{array}{l}24 . \\
24 . \\
24 . \\
21 . \\
20 . \\
20 . \\
19 . \\
19 . \\
18 . \\
13 . \\
12 . \\
11 .\end{array}$ \\
\hline \multicolumn{11}{|c|}{ IV. $(2.6-10$ Person-rem $)$} \\
\hline $\begin{array}{l}\text { San Onofre, W } \\
\text { Three Mile Isl, B } \\
\text { Ft. Calhoun, CE } \\
\text { Yankee Rowe, W } \\
\text { Monticello, CE } \\
\text { Farley, } \\
\text { St. Lucie, CE } \\
\text { Salem, } \\
\text { Point Beach, W } \\
\text { Prairie Island, W }\end{array}$ & $\begin{array}{l}68 \\
74,78 \\
73 \\
61 \\
71 \\
77,81 \\
76,8 \\
77,87 \\
71,72 \\
73,74\end{array}$ & $\begin{array}{l}0.28 \\
0.57 \\
0.13 \\
0.11 \\
5.20 \\
-.- \\
-.- \\
1.2 \\
0.12\end{array}$ & $\begin{array}{l}1.4 \\
1.4 \\
0.26 \\
0.07 \\
0.25 \\
-.- \\
0.03 \\
-.-33 \\
0.33 \\
1.40\end{array}$ & $\begin{array}{l}0.78 \\
2.00 \\
0.33 \\
0.20 \\
0.20 \\
0.65 \\
0.12 \\
0.18 \\
0.59\end{array}$ & $\begin{array}{l}1.80 \\
2.20 \\
0.49 \\
4.60 \\
0.20 \\
0.99 \\
1.00 \\
0.37 \\
0.12 \\
0.46\end{array}$ & $\begin{array}{l}0.52 \\
0.29 \\
0.39 \\
0.40 \\
0.19 \\
0.19 \\
0.76 \\
2.30 \\
0.25 \\
0.10\end{array}$ & $\begin{array}{l}3.10 \\
2.20 \\
0.48 \\
0.55 \\
0.16 \\
0.58 \\
0.51 \\
0.28 \\
0.20 \\
0.06\end{array}$ & $\begin{array}{l}1.80 \\
0.24 \\
6.10 \\
0.72 \\
0.14 \\
3.20 \\
0.62 \\
0.85 \\
0.19 \\
0.04\end{array}$ & $\begin{array}{l}0.51 \\
0.28 \\
0.36 \\
0.19 \\
0.19 \\
.154 \\
0.87 \\
0.58 \\
0.41 \\
0.07\end{array}$ & $\begin{array}{l}10 . \\
9.2 \\
8.5 \\
6.8 \\
6.5 \\
5.7 \\
4.5 \\
4.5 \\
2.9 \\
2.8\end{array}$ \\
\hline \multicolumn{11}{|l|}{ V. $(1-2.5$ Person-rem $)$} \\
\hline $\begin{array}{l}\text { Duane Arnold, GE } \\
\text { Palisades, CE } \\
\text { Sequoyah, } \\
\text { Ginna, W } \\
\text { Vermont Yankee, GE } \\
\text { Beaver Valley, " } \\
\text { Cooper, GE }\end{array}$ & $\begin{array}{l}75 \\
73 \\
20 \\
70 \\
72 \\
76 \\
74\end{array}$ & $\begin{array}{l}0.18 \\
0.62 \\
-- \\
0.28 \\
0.08 \\
-- \\
0.18\end{array}$ & $\begin{array}{l}0.32 \\
0.64 \\
-- \\
0.51 \\
0.11 \\
0.04 \\
0.39\end{array}$ & $\begin{array}{l}0.31 \\
0.63 \\
-- \\
0.14 \\
0.37 \\
0.40 \\
0.02\end{array}$ & $\begin{array}{l}0.87 \\
0.11 \\
0 . .3 \\
0.3 \\
0.24 \\
0.83 \\
0.05\end{array}$ & $\begin{array}{l}0.54 \\
0.12 \\
-- \\
0.11 \\
0.46 \\
0.17 \\
0.05\end{array}$ & $\begin{array}{l}0.11 \\
0.03 \\
0.22 \\
0.58 \\
0.06 \\
0.02 \\
0.07\end{array}$ & $\begin{array}{l}0.03 \\
0.16 \\
0.67 \\
0.15 \\
0.60 \\
0.11 \\
0.07\end{array}$ & $\begin{array}{l}0.01 \\
0.62 \\
1.2 \\
0.23 \\
0.06 \\
0.04 \\
0.17\end{array}$ & $\begin{array}{l}2.4 \\
2.9 \\
2.1 \\
2.1 \\
2.0 \\
1.6 \\
1.0\end{array}$ \\
\hline \multicolumn{11}{|l|}{ VI. (<1 Person-rem) } \\
\hline $\begin{array}{l}\text { Trojan, W } \\
\text { McCuire, } \\
\text { Maine Yankee, } \\
\text { Susquehanna, GE } \\
\text { LaSalle, GE } \\
\text { Summer, W }\end{array}$ & $\begin{array}{l}76 \\
81 \\
72 \\
82 \\
82 \\
82\end{array}$ & $\begin{array}{l}-- \\
-- \\
0.10 \\
-- \\
-- \\
=-\end{array}$ & $\begin{array}{l}0.02 \\
=- \\
0.06 \\
-- \\
=- \\
=-\end{array}$ & $\begin{array}{c}0.11 \\
--01 \\
-- \\
-- \\
-.\end{array}$ & $\begin{array}{c}0.04 \\
\cdots .03 \\
0.03 \\
\cdots \\
\cdots \\
\cdots\end{array}$ & $\begin{array}{c}0.05 \\
-- \\
0.04 \\
-- \\
--\end{array}$ & \begin{tabular}{l}
0.06 \\
-- \\
0.03 \\
\hdashline- \\
$\because-$
\end{tabular} & $\begin{array}{l}0.20 \\
0.26 \\
0.01 \\
-. \\
-.\end{array}$ & $\begin{array}{l}0.17 \\
0.39 \\
0.01 \\
0.21 \\
<0.01 \\
<0.01\end{array}$ & $\begin{array}{l}0.65 \\
0.59 \\
0.29 \\
0.21 \\
0.01 \\
0.01\end{array}$ \\
\hline TO & & 1300 & 70 & 00 & 640 & 10 & 180 & 60 & 130 & 100 \\
\hline
\end{tabular}

Note: Only the doses from the TMI accident, 1979, are excluded. 
SITE SUMMARIES

1982

2.1 
Site: ARKANSAS ONE

POPE COUNTY, ARKANSAS

Location: $\quad N 35.3100^{\circ}$ W $93.2308^{\circ}$

POPULATION DATA

Total Population Within 2-to-80-km Region: 1.8 E5

Major Metropolitan Centers Within Region:

Center

Russellville

Conway
Population

14,000

20,000
Location

$10 \mathrm{~km} \quad \mathrm{E}$

$76 \mathrm{~km} \quad \mathrm{ESE}$

\section{SITE SPECIFIC DATA - AIRBORNE PATHWAYS}

Average Annual State Production

of Crops and Animal Products

In $80-k m$ Radius Circle

Regional Productivity Factor:

Animal Grazing Factor:

Meteorology Period of Record: 1 JAN 75 - 31 DEC 75 Recovery: 97\%
Veg: $5.8 E 6$ kilogram

Milk: 4.8E7 liter

Meat: $7.2 E 7$ kilogram

1

0.7

SITE SPECIFIC OATA - WATERBORNE PATHWAYS via ARKANSAS RIVER

Average River Flow at Site: $36,000 \mathrm{ft}^{3} / \mathrm{s}$

Drinking Water:

Exposed Population: None

Fish:

Edible Harvest: $1.4^{(\mathrm{a})} \mathrm{kg} / \mathrm{yr}$ 0ilution Factor: 1

(a) Average individual consumption rates as given in the FES (1973) were used in lieu of catch data. 
POPULATION DOSE-COMMITMENT ESTIMATES AND

AVERAGE INDIVIDUAL DOSE-COMMITMENT HISTOGRAM FOR

\section{ARKANSAS ONE 1 AND 2}

Dose Commitments (person-rem) from Liquid Pathways

\begin{tabular}{|c|c|c|c|c|c|}
\hline & Total Body & GI-LLI & Thyroid & Bone & Liver \\
\hline $\begin{array}{l}\text { Infant } \\
\text { Child } \\
\text { Teen } \\
\text { Adult }\end{array}$ & $\begin{array}{l}0.0 \mathrm{E}+00 \\
1.9 \mathrm{E}-01 \\
3.6 \mathrm{E}-01 \\
3.9 \mathrm{E}+00\end{array}$ & $\begin{array}{l}0.0 \mathrm{E}+00 \\
6.1 \mathrm{E}-02 \\
1.3 \mathrm{E}-01 \\
1.1 \mathrm{E}+00\end{array}$ & $\begin{array}{l}0.0 \mathrm{E}+00 \\
3.9 \mathrm{E}-02 \\
2.8 \mathrm{E}-02 \\
1.9 \mathrm{E}-01\end{array}$ & $\begin{array}{l}0.0 \mathrm{E}+00 \\
9.4 \mathrm{E}-01 \\
5.6 \mathrm{E}-01 \\
3.3 \mathrm{E}+00\end{array}$ & $\begin{array}{l}0.0 \mathrm{E}+00 \\
1.1 \mathrm{E}+00 \\
9.1 \mathrm{E}-01 \\
5.4 \mathrm{E}+00\end{array}$ \\
\hline TOTAL & 4. $4 E+00$ & 1. $3 \mathrm{E}+00$ & $2.5 \mathrm{E}-01$ & $4.8 E+00$ & $7.4 \mathrm{E}+00$ \\
\hline
\end{tabular}

Dose Commitments (person-rem) from Airborne Pathways

Total Body GI-LLI Thyroid Bone Liver Lung

$\begin{array}{lllllll}\text { Infant } & 1.4 \mathrm{E}-03 & 1.4 \mathrm{E}-03 & 2.3 \mathrm{E}-03 & 1.4 \mathrm{E}-03 & 1.4 \mathrm{E}-03 & 1.5 \mathrm{E}-03 \\ \text { Child } & 1.6 \mathrm{E}-02 & 1.6 \mathrm{E}-02 & 2.1 \mathrm{E}-02 & 1.6 \mathrm{E}-02 & 1.6 \mathrm{E}-02 & 1.7 \mathrm{E}-02 \\ \text { Teen } & 1.2 \mathrm{E}-02 & 1.2 \mathrm{E}-02 & 1.4 \mathrm{E}-02 & 1.1 \mathrm{E}-02 & 1.2 \mathrm{E}-02 & 1.4 \mathrm{E}-02 \\ \text { Adult } & 7.0 \mathrm{E}-02 & 7.0 \mathrm{E}-02 & 7.7 \mathrm{E}-02 & 6.9 \mathrm{E}-02 & 7.0 \mathrm{E}-02 & 7.6 \mathrm{E}-02 \\ \text { TOTAL } & 9.9 \mathrm{E}-02 & 9.9 \mathrm{E}-02 & 1.1 \mathrm{E}-01 & 9.7 \mathrm{E}-02 & 9.9 \mathrm{E}-02 & 1.1 \mathrm{E}-01\end{array}$

Production/Consumption factors: ${ }^{(a)}$

Produce: <1 Milk: 2.0 Meat: 4.9

FRACTION DF POPULATION RECEIVING AN AVERAGE INDIVIDUAL

TOTAL-BODY ODSE COMMITMENT FROM AIRBDRNE PATHWAYS

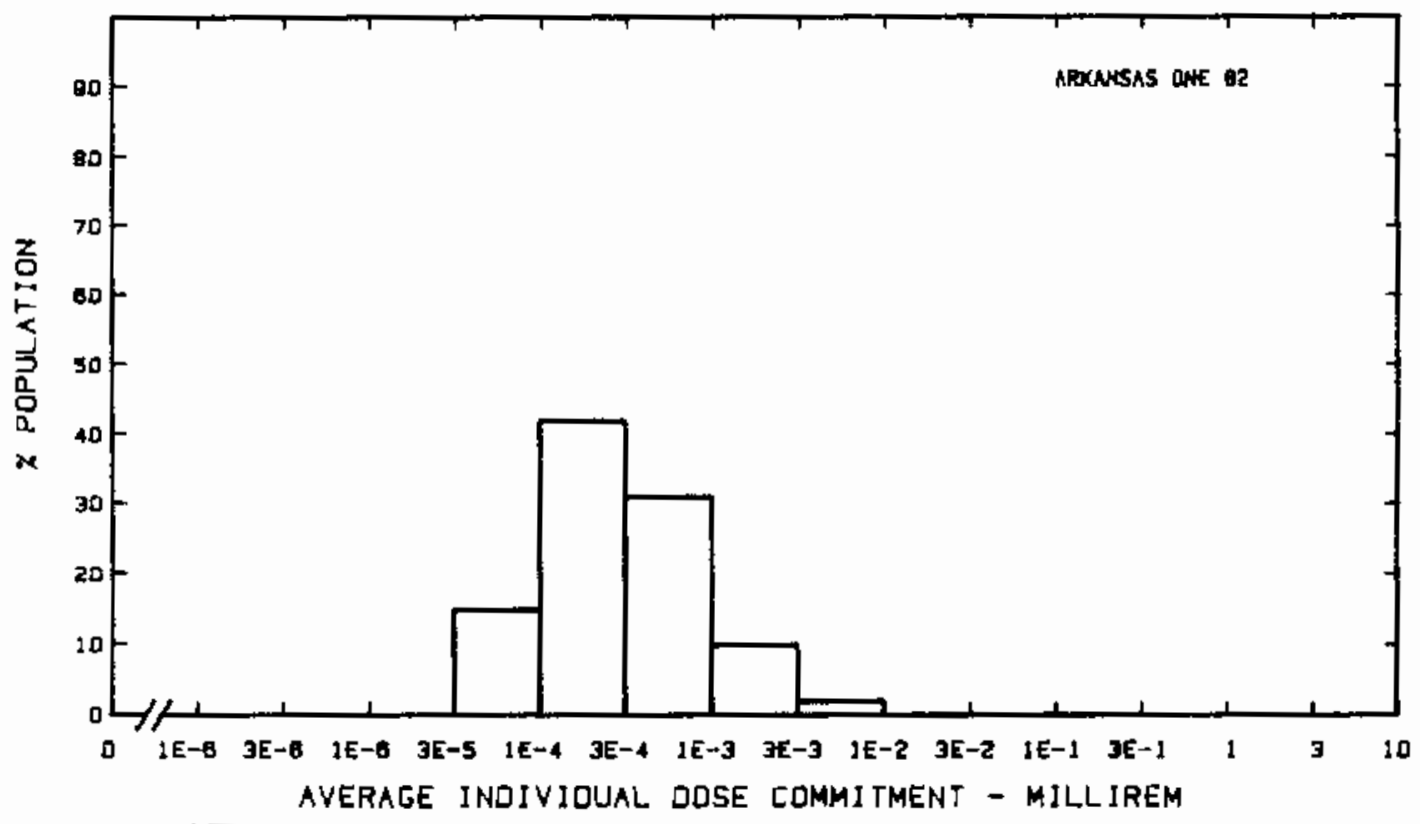

(a) See Appendix A, Page A-5, for explanation of this ratio. 
Site: BEAVER VALLEY

Location: N $40.6219^{\circ} \quad$ W $80.4339^{\circ}$
SHIPPINGPORT, PENNSYLVANIA

POPULATION DATA

Total Population Within 2-to-80-km Region: 3.6E6

Major Metropolitan Centers Within Region:

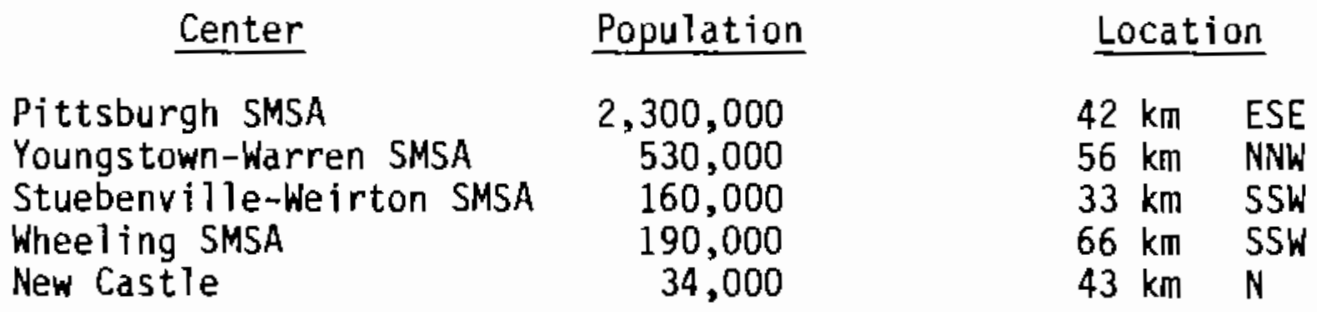

SITE SPECIFIC OATA - AIRBORNE PATHWAYS

Average Annual State Production

of Crops and Animal Products

In 80-km Radius Circle

Regional Productivity Factor:

Animal Grazing Factor:
Veg: $5.3 \mathrm{E} 7 \mathrm{kj}$ logram

Mijk: 5.3E8 liter

Meat: $5.4 E 7$ kilogram

1

0.5

Meteorology Period of Record: 1 JAN 77 - 31 DEC 77 Recovery: 92\%

SITE SPECIFIC DATA - WATERBORNE PATHWAYS via OHIO RIVER

Average River Flow at Site: $30,000 \mathrm{ft}^{3} / 5$

Drinking Water:

Exposed Population: $(6,200$
Dilution Factor: 12

Fish:

Edible Harvest: $410 \mathrm{~kg} / \mathrm{yr}$ Dilution Factior: 1

(a) This factor accounts for the incomplete dilution of plant effluent by river at point of drinking water intake at Midland. 


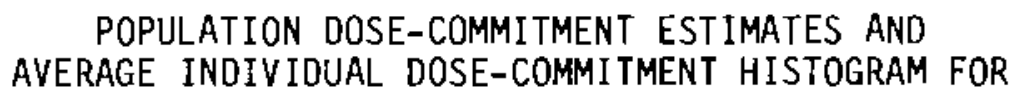

\section{BEAVER VALLEY}

Dose Commitments (person-rem) from Liquid Pathways

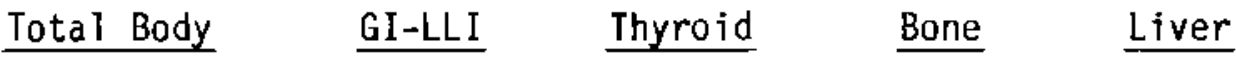

$\begin{array}{llllll}\text { Infant } & 4.1 \mathrm{E}-04 & 4.0 \mathrm{E}-04 & 1.3 \mathrm{E}-03 & 4.4 \mathrm{E}-05 & 4.4 \mathrm{E}-04 \\ \text { Child } & 4.6 \mathrm{E}-03 & 4.6 \mathrm{E}-03 & 1.1 \mathrm{E}-02 & 4.9 \mathrm{E}-04 & 4.8 \mathrm{E}-03 \\ \text { Teen } & 1.8 \mathrm{E}-03 & 1.9 \mathrm{E}-03 & 3.6 \mathrm{E}-03 & 1.3 \mathrm{E}-04 & 1.8 \mathrm{E}-03 \\ \text { Adult } & 1.5 \mathrm{E}-02 & 1.7 \mathrm{E}-02 & 2.8 \mathrm{E}-02 & 8.8 \mathrm{E}-04 & 1.5 \mathrm{E}-02 \\ \text { TOTAL } & 2.2 \mathrm{E}-02 & 2.4 \mathrm{E}-02 & 4.4 \mathrm{E}-02 & 1.5 \mathrm{E}-03 & 2.2 \mathrm{E}-02\end{array}$

Dose Commitments (person-rem) from Airborne Pathways

Total Body GI-LLI Thyroid Bone Liver $\underline{\text { Lung }}$

$\begin{array}{lllllll}\text { Infant } & 2.0 \mathrm{E}-04 & 1.9 \mathrm{E}-04 & 8.4 \mathrm{E}-03 & 2.3 \mathrm{E}-04 & 2.2 \mathrm{E}-04 & 2.3 \mathrm{E}-04 \\ \text { Child } & 2.3 \mathrm{E}-03 & 2.2 \mathrm{E}-03 & 5.0 \mathrm{E}-02 & 2.7 \mathrm{E}-03 & 2.3 \mathrm{E}-03 & 2.7 \mathrm{E}-03 \\ \text { Teen } & 1.6 \mathrm{E}-03 & 1.6 \mathrm{E}-03 & 2.2 \mathrm{E}-02 & 1.9 \mathrm{E}-03 & 1.6 \mathrm{E}-03 & 2.2 \mathrm{E}-03 \\ \text { Adult } & 9.7 \mathrm{E}-03 & 9.6 \mathrm{E}-03 & 8.3 \mathrm{E}-02 & 1.1 \mathrm{E}-02 & 9.6 \mathrm{E}-03 & 1.2 \mathrm{E}-02 \\ \text { TOTAL } & 1.4 \mathrm{E}-02 & 1.4 \mathrm{E}-02 & 1.6 \mathrm{E}-01 & 1.6 \mathrm{E}-02 & 1.4 \mathrm{E}-02 & 1.7 \mathrm{E}-02\end{array}$

Production/Consumption factors:

Produce: 1 Milk: 1.1 Meat: 1

FRACTION DF POPULATION RECEIVING AN AVERAGE INDIVIDUAL TOTAL-BODY OOSE COMMITMENT FROM AIREORNE PATHWAYS

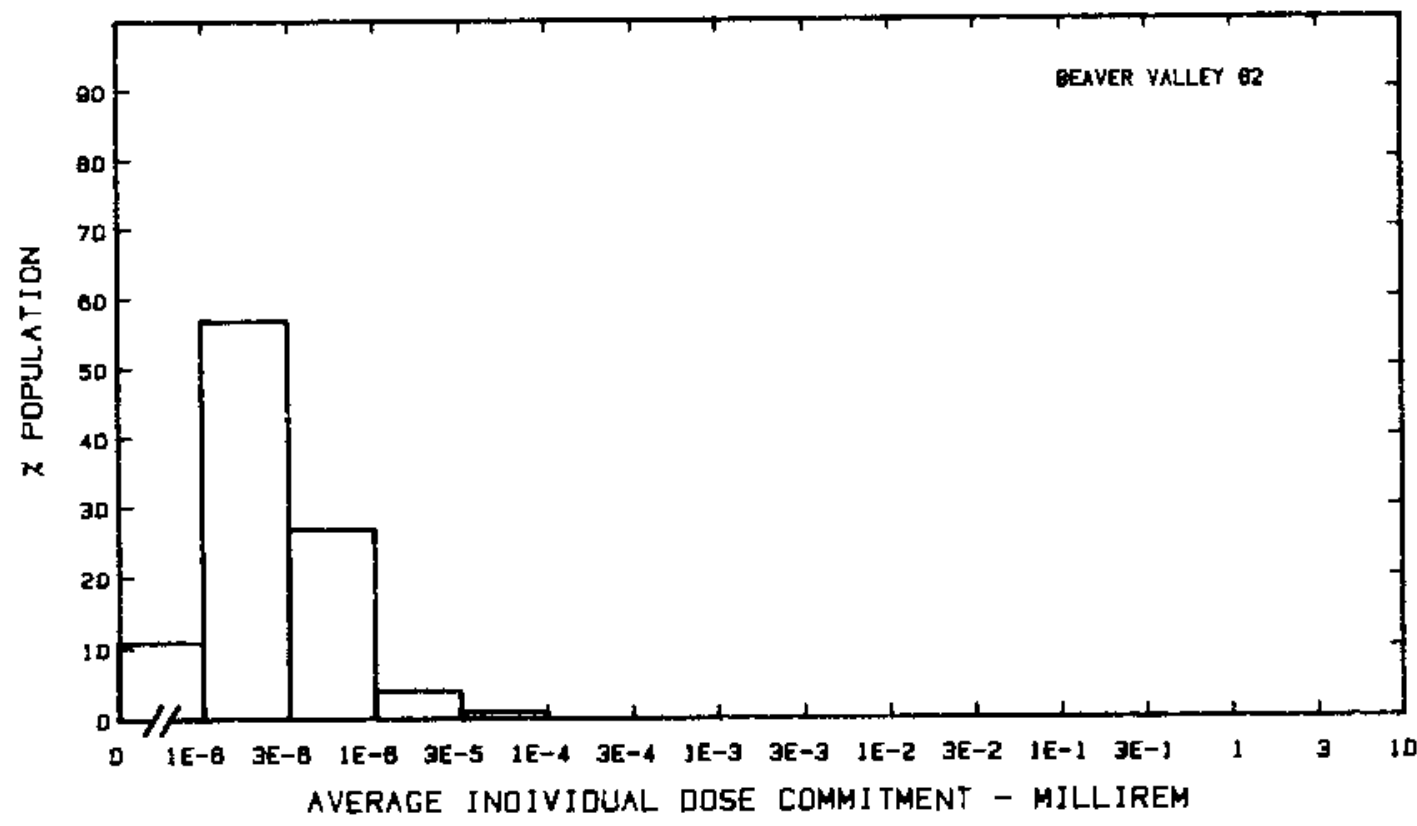


Site: BIG ROCK POINT

CHARLEVOIX COUNTY, MICHIGAN
Location:
N $45.3592^{\circ}$
W $85.1947^{\circ}$

POPULATION DATA

Total Population Within 2-to-80-km Region: 1.7 E5

Major Metropolitan Centers Within Region:

\begin{tabular}{lccc} 
Center & Population & \multicolumn{2}{c}{ Location } \\
\cline { 2 - 4 } & & & \\
Traverse City & 16,000 & $75 \mathrm{~km}$ & SSW \\
Petoskey & 6,100 & $18 \mathrm{~km}$ & E \\
Cheboygan & 5,100 & $65 \mathrm{~km}$ & ENE
\end{tabular}

SITE SPECIFIC DATA - AIRBORNE PATHWAYS

Average Annual State Production

of Crops and Animal Products

In 80-km Radius Circle

Regional Productivity Factor:

Animal Grazing Factor:

Meteorology Period of Record: 9 FEB 61 - 8 FEB 63 Recovery: 85\%
Veg: $6.9 \mathrm{E} 7 \mathrm{kilogram}$

Milk: $2.9 \mathrm{EE}$ liter

Meat: $4.5 \mathrm{E} 7 \mathrm{kilogram}$

0.5

0.5

SITE SPECIFIC DATA - WATERBORNE PATHWAYS via LAKE MICHIGAN

Average Dilution Flow

from Plant: $94 \mathrm{ft}^{3} / \mathrm{s}$

Drinking Water:

Exposed Population: $31, \varphi p 9^{(a)}$

Fish:

Edible Harvest: (c) $\mathrm{kg} / \mathrm{yr}$

Dilution Factor: 0.01

(a) Population exposed to contaminated drinking water derived from information obtained from J. Hennigan, Oivision of Radiation Health, Bureau of Environment and Health, Michigan Department of Public Heal th.

(b) Drinking water dilution factor estimated by averaging dilution factor derived from Figure 6B-5, Vol. 1 of WASH-1258 (1973) suitably weighted for population.

(c) Generic consumption rate used (Table $A-1$ ), 
POPULATION DOSE-COMMITMENT ESTIMATES AND

AVERAGE INDIVIDUAL DOSE-COMMITMENT HISTOGRAM FOR

\section{BIG ROCK POINT}

Dose Commitments (person-rem) from Liquid Pathways

Total Body GI-LLI $\underline{\text { Thyroid }}$ Bone Liver

$\begin{array}{llllll}\text { Infant } & 1.1 \mathrm{E}-06 & 2.0 \mathrm{E}-07 & 4.0 \mathrm{E}-08 & 4.6 \mathrm{E}-06 & 1.8 \mathrm{E}-06 \\ \text { Child } & 4.4 \mathrm{E}-02 & 4.5 \mathrm{E}-03 & 4.2 \mathrm{E}-06 & 2.9 \mathrm{E}-01 & 2.7 \mathrm{E}-01 \\ \text { Teen } & 8.0 \mathrm{E}-02 & 9.3 \mathrm{E}-03 & 3.6 \mathrm{E}-06 & 1.7 \mathrm{E}-01 & 2.2 \mathrm{E}-01 \\ \text { Adult } & 8.7 \mathrm{E}-01 & 8.0 \mathrm{E}-02 & 2.9 \mathrm{E}-05 & 1.0 \mathrm{E}+00 & 1.3 \mathrm{E}+00 \\ \text { TOTAL } & 1.0 \mathrm{E}+00 & 9.4 \mathrm{E}-02 & 3.7 \mathrm{E}-05 & 1.5 \mathrm{E}+00 & 1.8 \mathrm{E}+00\end{array}$

Dose Commitments (person-rem) from Airborne Pathways

Total Body GI-LLI Thyroid Bone Liver Lung

$\begin{array}{lllllll}\text { Infant } & 2.3 \mathrm{E}-03 & 2.3 \mathrm{E}-03 & 2.5 \mathrm{E}-03 & 2.3 \mathrm{E}-03 & 2.3 \mathrm{E}-03 & 2.4 \mathrm{E}-03 \\ \text { Child } & 2.6 \mathrm{E}-02 & 2.6 \mathrm{E}-02 & 2.8 \mathrm{E}-02 & 2.6 \mathrm{E}-02 & 2.6 \mathrm{E}-02 & 2.7 \mathrm{E}-02 \\ \text { Teen } & 1.9 \mathrm{E}-02 & 1.9 \mathrm{E}-02 & 2.0 \mathrm{E}-02 & 1.9 \mathrm{E}-02 & 1.9 \mathrm{E}-02 & 2.0 \mathrm{E}-02 \\ \text { Adult } & 1.1 \mathrm{E}-01 & 1.1 \mathrm{E}-01 & 1.2 \mathrm{E}-01 & 1.1 \mathrm{E}-01 & 1.1 \mathrm{E}-01 & 1.2 \mathrm{E}-01 \\ \text { TOTAL } & 1.6 \mathrm{E}-01 & 1.6 \mathrm{E}-01 & 1.7 \mathrm{E}-01 & 1.6 \mathrm{E}-01 & 1.6 \mathrm{E}-01 & 1.7 \mathrm{E}-01\end{array}$

Production/Consumption factors:

Produce: $1.0 \quad$ Milk: $6.5 \quad$ Meat: 1.7

FRACTION OF POPULATION RECEIVING AN AVERAGE INOIVIDUAL TOTAL-BODY DOSE COMMITMENT FROM AIREORNE PATHWAYS

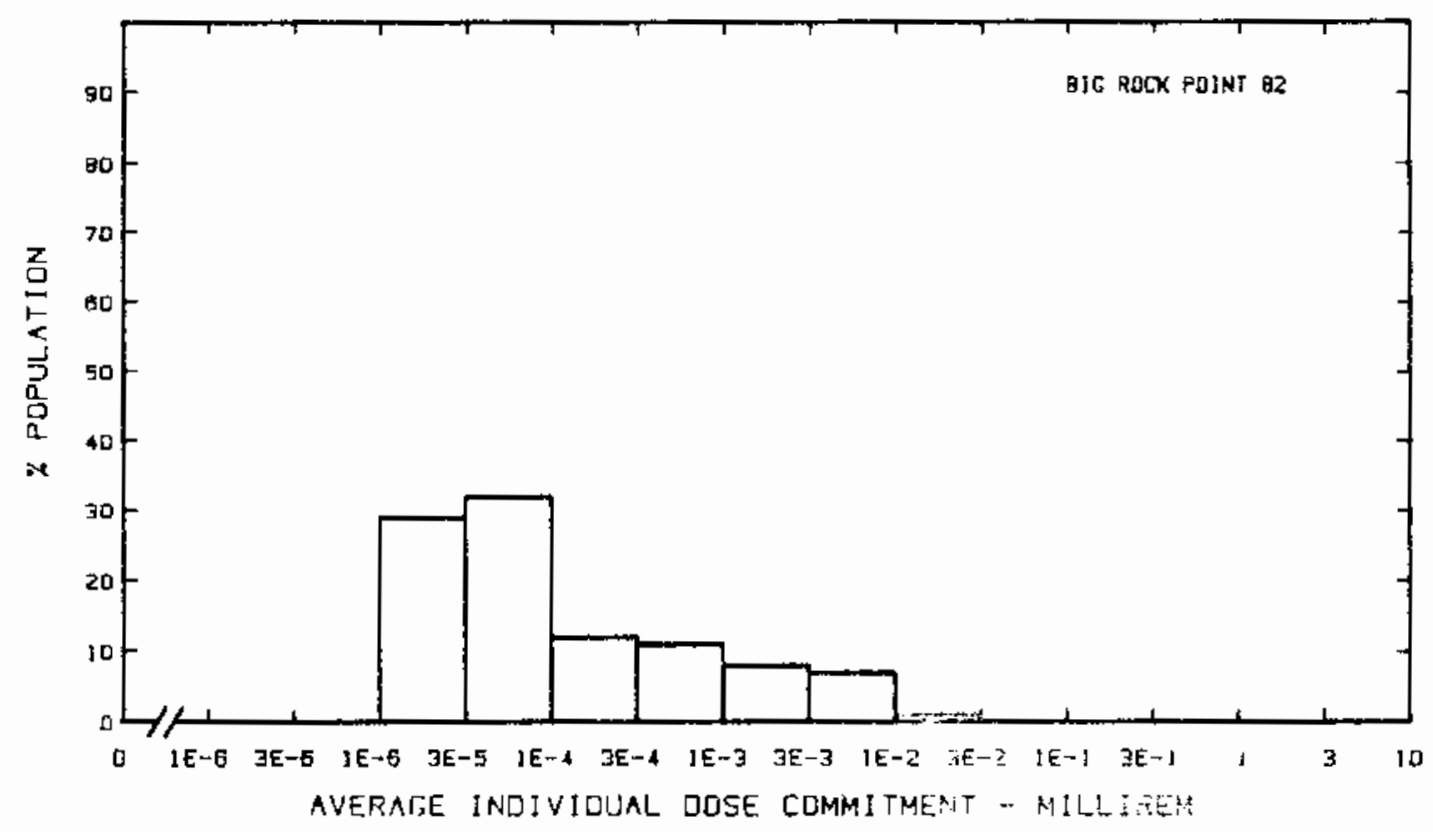


Site: BROWNS FERRY

DECATIJR, ALABAMA

Location: $\quad \mathrm{N} 34.7042^{\circ}$ W $87.1136^{\circ}$

POPULATION DATA

Total Population Within 2-to-80-km Region: 7.2E5

Major Metropolitan Centers Within Region:

Center

HuntsviTle SMSA

Florence SMSA

Decatur

Athens

Cul 1 man
Population

310,000

140,000

42,000

15,000

13,000
Location

$49 \mathrm{~km} \quad \mathrm{E}$

$52 \mathrm{~km} \quad \mathrm{WNW}$

$16 \mathrm{~km} \quad \mathrm{SE}$

$17 \mathrm{~km} \quad \mathrm{NE}$

$64 \mathrm{~km} \quad \mathrm{SSE}$

SITE SPECIFIC DATA - AIRBORNE PATHWAYS

Average Annual State Production

of Crops and Animal Products

In 80-km Radius Circle

Regional Productivity Factor:

Animal Grazing Factor:

Meteorology Period of Record: 1 JAN 74 - 31 DEC 75 Recovery: 94\%
Veg: $1.7 \mathrm{E} 7 \mathrm{kilog}$ ram

Milk: $5.7 E 7$ liter

Meat: $8.6 \mathrm{E} 7$ kilogram

1

0.7

SITE SPECIFIC DATA - WATERBORNE PATHWAYS via TENNESSEE RIVER at WHEELER LAKE

Average River Flow

at Site: $45,000 \mathrm{ft}^{3} / \mathrm{s}$

Drinking Water:

Exposed Population: 25,000

Dilution Factor: 1

Fish:

Edible Harvest: $1.6 \mathrm{E} 6 \mathrm{~kg} / \mathrm{yr}$

Dilution Factor: 1 


\section{POPULATION OOSE-COMMITMENT ESTIMATES AND \\ AVERAGE INDIVIDUAL OOSE-COMMITMENT HISTOGRAM FOR \\ BROWNS FERRY 1,2 AND 3}

Dose Commitments (person-rem) from Liquid Pathways

Total Body GI-LLI Thyroid Bone Liver

$\begin{array}{llllll}\text { Infant } & 2.0 \mathrm{E}-04 & 1.7 \mathrm{E}-04 & 4.6 \mathrm{E}-03 & 5.2 \mathrm{E}-04 & 5.1 \mathrm{E}-04 \\ \text { Child } & 2.0 \mathrm{E}-01 & 6.2 \mathrm{E}-02 & 5.7 \mathrm{E}-02 & 5.8 \mathrm{E}-01 & 7.4 \mathrm{E}-01 \\ \text { Teen } & 2.7 \mathrm{E}-01 & 1.3 \mathrm{E}-01 & 2.8 \mathrm{E}-02 & 3.5 \mathrm{E}-01 & 6.4 \mathrm{E}-01 \\ \text { Adult } & 2.7 \mathrm{E}+00 & 1.2 \mathrm{E}+00 & 1.9 \mathrm{E}-01 & 2.1 \mathrm{E}+00 & 3.9 \mathrm{E}+00 \\ \text { TOTAL } & 3.1 \mathrm{E}+00 & 1.4 \mathrm{E}+00 & 2.8 \mathrm{E}-01 & 3.0 \mathrm{E}+00 & 5.3 \mathrm{E}+00\end{array}$

Oose Comnitments (person-rem) from Airborne Pathways Total Body GI-LLI Thyroid Bone Liver $\underline{\text { Lung }}$

$\begin{array}{lllllll}\text { Infant } & 4.4 \mathrm{E}-01 & 4.4 \mathrm{E}-01 & 4.7 \mathrm{E}-01 & 4.4 \mathrm{E}-01 & 4.4 \mathrm{E}-01 & 4.5 \mathrm{E}-01 \\ \text { Child } & 4.9 \mathrm{E}+00 & 4.9 \mathrm{E}+00 & 5.1 \mathrm{E}+00 & 4.9 \mathrm{E}+00 & 4.9 \mathrm{E}+00 & 5.1 \mathrm{E}+00 \\ \text { Teen } & 3.6 \mathrm{E}+00 & 3.6 \mathrm{E}+00 & 3.7 \mathrm{E}+00 & 3.6 \mathrm{E}+00 & 3.6 \mathrm{E}+00 & 3.8 \mathrm{E}+00 \\ \text { Adult } & 2.2 \mathrm{E}+01 & 2.2 \mathrm{E}+01 & 2.2 \mathrm{E}+01 & 2.2 \mathrm{E}+01 & 2.2 \mathrm{E}+01 & 2.2 \mathrm{E}+01 \\ \text { TOTAL } & 3.1 \mathrm{E}+01 & 3.1 \mathrm{E}+01 & 3.1 \mathrm{E}+01 & 3.1 \mathrm{E}+01 & 3.1 \mathrm{E}+01 & 3.2 \mathrm{E}+01\end{array}$

Production/Consumption factors:

Produce: 1 Milk: 1 Meat: 1.5

FRACTION OF POPULATION RECEIVING AN AVERAGE INDIVIDUAL TOTAL-BODY DOSE COMMITMENT FROM AIRBORNE PATHWAYS

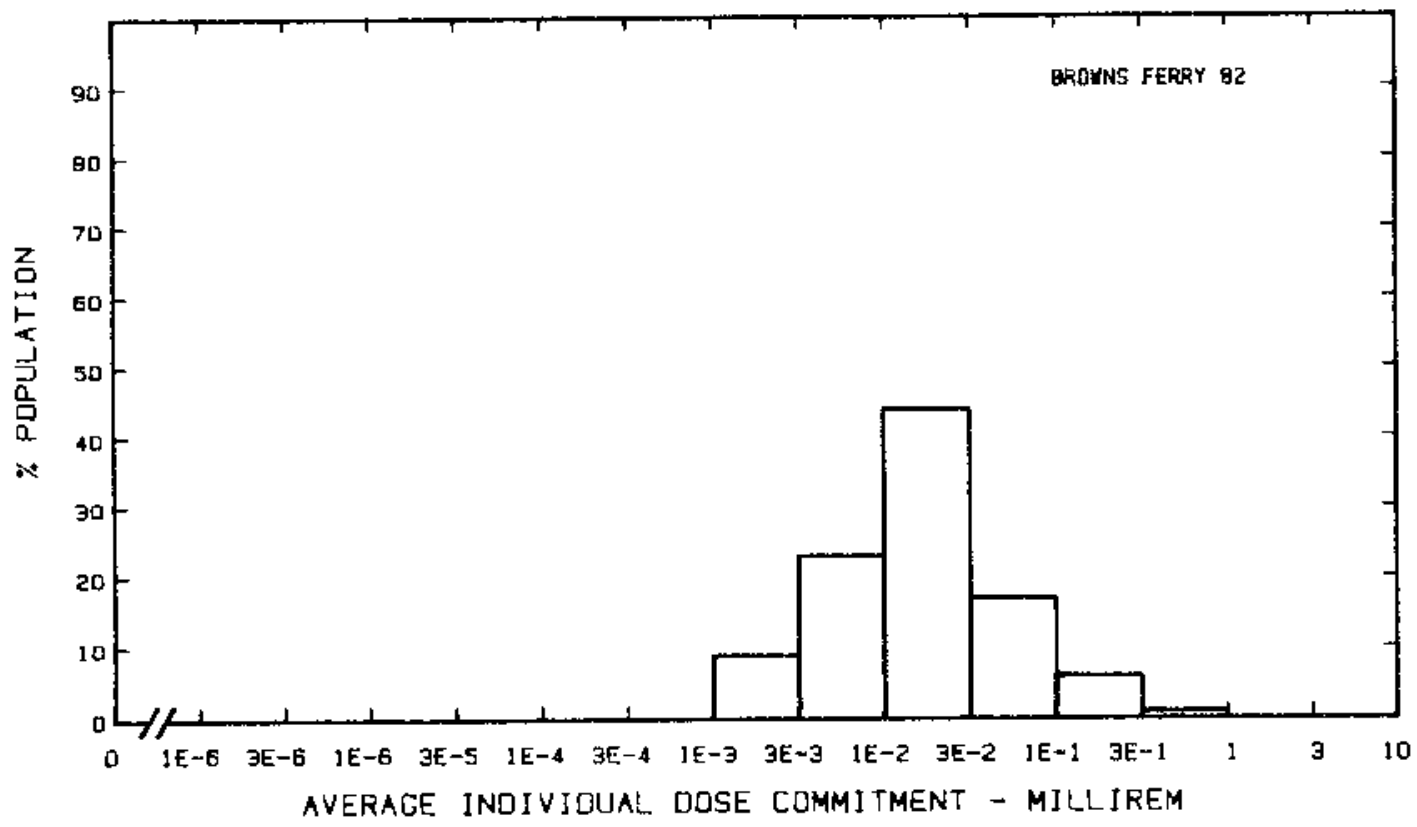


Location: N $33.9583^{\circ}$ W $78.0106^{\circ}$

POPULATION DATA

Total Population Within 2-to-80-km Region: 2.2E5

Major Metropolitan Centers Within Region:

Center Population Location

Wilmington SMSA $\quad 140,000 \quad 32 \mathrm{~km}$ NNE

Whiteville $\quad 5,600 \quad 75 \mathrm{~km}$ WNW

N. Myrtle Beach $\quad 4,000 \quad 65 \mathrm{~km}$ WSW

SITE SPECIFIC DATA - AIRBORNE PATHWAYS

Average Annual State Production

of Crops and Animal Products

In $80-\mathrm{km}$ Radius Circle

Veg: $2.6 E 7$ kilogram

Milk: $1.0 \mathrm{E} 8$ liter

Regional Productivity Factor:

Animal Grazing Factor:

Meat: $5.8 \mathrm{E} 7$ kilogram

Meteorology Period of Record: 1 JAN 75 - 31 DEC 75 Recovery: 93\%

SITE SPECIFIC DATA - WATERBORNE PATHWAYS via ATLANTIC OCEAN

Average Dilution Flow

from Plant: $1,300 \mathrm{ft}^{3} / \mathrm{s}$

Fish:

Edible Harvest: $2.1 \mathrm{E5}^{(\mathrm{a})} \mathrm{kg} / \mathrm{yr}$

Dilution Factor: 0.001

Invertebrates:

Edible Harvest: $1.1 \mathrm{E5}^{(\mathrm{a})} \mathrm{kg} / \mathrm{yr}$

Dilution Factor: 0.002

(a) Fish and invertebrate harvests together total harvest given in FES $\langle 1973\rangle$. 


\section{POPULATION DOSE-COMMITMENT ESTIMATES AND \\ AVERAGE INDIVIDUAL DOSE-COMMITMENT HISTOGRAM FOR \\ BRUNSWICK 1 AND 2}

Dose Commitments (person-rem) from Liquid Pathways

\begin{tabular}{|c|c|c|c|c|c|}
\hline & Total Body & GI-LLI & Thyroid & Bone & Liver \\
\hline $\begin{array}{l}\text { Infant } \\
\text { Child } \\
\text { Teen } \\
\text { Adult }\end{array}$ & $\begin{array}{l}0.0 \mathrm{E}+00 \\
3.0 \mathrm{E}-04 \\
2.2 \mathrm{E}-04 \\
1.4 \mathrm{E}-03\end{array}$ & $\begin{array}{l}0.0 \mathrm{E}+00 \\
4.8 \mathrm{E}-04 \\
9.8 \mathrm{E}-04 \\
8.6 \mathrm{E}-03\end{array}$ & $\begin{array}{l}0.0 \mathrm{E}+00 \\
2.4 \mathrm{E}-04 \\
1.7 \mathrm{E}-04 \\
1.1 \mathrm{E}-03\end{array}$ & $\begin{array}{l}0.0 \mathrm{E}+00 \\
2.5 \mathrm{E}-04 \\
1.6 \mathrm{E}-04 \\
1.0 \mathrm{E}-03\end{array}$ & $\begin{array}{l}0.0 E+00 \\
3.9 E-04 \\
3.4 E-04 \\
2.1 E-03\end{array}$ \\
\hline TOTAL & $2.0 \mathrm{E}-03$ & $1.0 \mathrm{E}-02$ & $1.5 E-03$ & $1.4 \mathrm{E}-03$ & $2.8 \mathrm{E}-03$ \\
\hline
\end{tabular}

Dose Commitments (person-rem) from Airborne Pathways

\begin{tabular}{lccccccc} 
& Total Body & GI-LLI & Thyroid & Bone & Liver & Lung \\
\cline { 2 - 3 } Infant & $2.0 \mathrm{E}-01$ & $2.0 \mathrm{E}-01$ & $3.2 \mathrm{E}-01$ & $2.0 \mathrm{E}-01$ & $2.0 \mathrm{E}-01$ & $2.0 \mathrm{E}-01$ \\
Child & $2.2 \mathrm{E}+00$ & $2.2 \mathrm{E}+00$ & $3.0 \mathrm{E}+00$ & $2.2 \mathrm{E}+00$ & $2.2 \mathrm{E}+00$ & $2.2 \mathrm{E}+00$ \\
Teen & $1.6 \mathrm{E}+00$ & $1.6 \mathrm{E}+00$ & $1.9 \mathrm{E}+00$ & $1.6 \mathrm{E}+00$ & $1.6 \mathrm{E}+00$ & $1.7 \mathrm{E}+00$ \\
Adult & $9.6 \mathrm{E}+00$ & $9.6 \mathrm{E}+01$ & $1.1 \mathrm{E}+01$ & $9.6 \mathrm{E}+01$ & $9.6 \mathrm{E}+00$ & $9.9 \mathrm{E}+00$ \\
TOTAL & $1.4 \mathrm{E}+01$ & $1.4 \mathrm{E}+01$ & $1.6 \mathrm{E}+01$ & $1.4 \mathrm{E}+01$ & $1.4 \mathrm{E}+01$ & $1.4 \mathrm{E}+01$
\end{tabular}

Production/Consumption factors:

Produce: 1 Milk: 1.1 Meat: 1.0

FRACTION OF POPULATION RECEIYING AN AYERAGE INDIVIJUAL TOTAL-BODY DDSE COMHITMENT FROH AIRBDRNE PATHWAYS

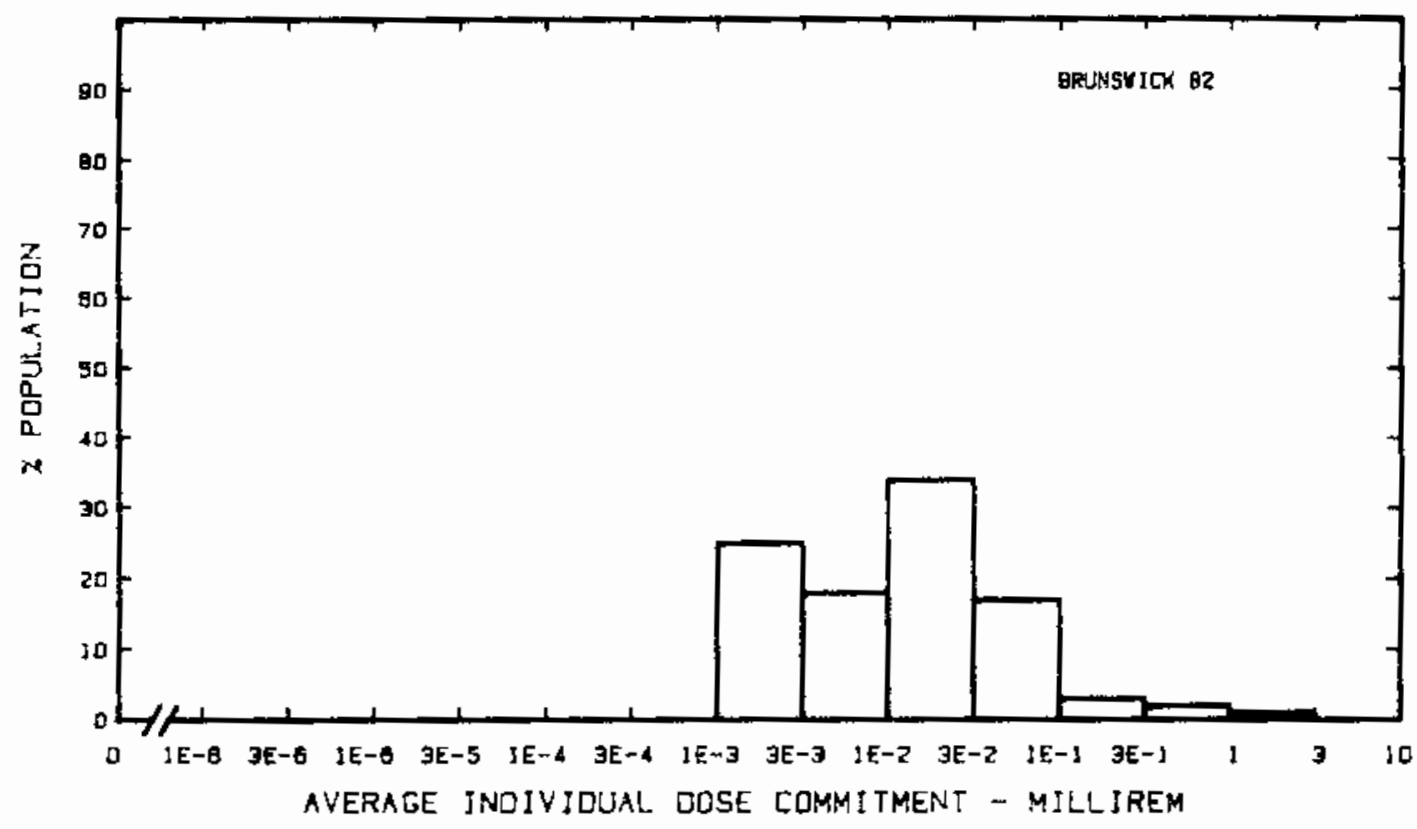


Site: CALVERT CLIFFS

LUSBY, MARYLAND

Location: N $38.4347^{\circ} \quad$ W $76.4419^{\circ}$

POPULATION DATA

Total Population Within 2-to-80-km Region: 2.6E6

Major Metropolitan Centers Within Region:

\begin{tabular}{lrrrr}
\multicolumn{1}{c}{ Center } & Population & \multicolumn{2}{c}{ Location } \\
\cline { 2 - 4 } & & $73 \mathrm{~km}$ & $\mathrm{NW}$ \\
Washington, DC SMSA (3/4) & $3,100,000$ & 34,000 & $71 \mathrm{~km}$ & NNW \\
Bowie & 32,000 & $61 \mathrm{~km}$ & $\mathrm{~N}$ \\
Annapolis & 9,800 & $46 \mathrm{~km}$ & WNW \\
Waldorf & 16,000 & $75 \mathrm{~km}$ & E \\
Salisbury & 12,000 & $32 \mathrm{~km}$ & ENE \\
Cambridge & & &
\end{tabular}

SITE SPECIFIC DATA - AIRBORNE PATHWAYS

Average Annual State Production

of Crops and Animal Products

In $80-\mathrm{km}$ Radius Circle

Regional Productivity Factor:

Animal Grazing Factor:

Meteorology Period of Record: 1 JAN 75 - 31 DEC 75
Veg: $4.5 \mathrm{E} 7 \mathrm{ki}$ logram

Milk: 5. OE8 liter

Meat: $6.2 \mathrm{E} 7 \mathrm{kjlogram}$

0.6

0.6

Recovery: $96 \%$

SITE SPECIFIC DATA - WATERBORNE PATHWAYS via CHESAPEAKE BAY

Average Dilution Flow

from Plant: 2,600 $\mathrm{ft}^{3} / \mathrm{s}$

Fish:

Edible Harvest: $1.0 E 7 \mathrm{~kg} / \mathrm{yr}$
Dilution Factor: $0.062 \mathrm{~g}$

Invertebrates:

Edible Harvest: $7.4 \mathrm{E} 6 \mathrm{~kg} / \mathrm{yr}$

Dilution Factor: $0.062^{(a)}$

(a) Dilutions given in FES (1973). 
POPULATION DOSE-COMMITMENT ESTIMATES ANO

AVERAGE INDIVIDUAL OOSE-COMMITMENT HISTOGRAM FOR

CALVERT CLIFFS 1 ANO 2

Oose Commitments (person-rem) from Liquid Pathways

Total Body GI-LLI Thyroid Bone Liver

$\begin{array}{llllll}\text { Infant } & 0.0 \mathrm{E}+00 & 0.0 \mathrm{E}+00 & 0.0 \mathrm{E}+00 & 0.0 \mathrm{E}+00 & 0.0 \mathrm{E}+00 \\ \text { Child } & 1.5 \mathrm{E}-01 & 1.6 \mathrm{E}+00 & 7.1 \mathrm{E}-01 & 3.9 \mathrm{E}-01 & 3.5 \mathrm{E}-01 \\ \text { Teen } & 1.7 \mathrm{E}-01 & 3.4 \mathrm{E}+00 & 5.0 \mathrm{E}-01 & 2.6 \mathrm{E}-01 & 2.9 \mathrm{E}-01 \\ \text { Adult } & 1.6 \mathrm{E}+00 & 3.0 \mathrm{E}+01 & 3.3 \mathrm{E}+00 & 1.7 \mathrm{E}+00 & 1.8 \mathrm{E}+00 \\ \text { TOTAL } & 1.9 \mathrm{E}+00 & 3.5 \mathrm{E}+01 & 4.5 \mathrm{E}+00 & 2.3 \mathrm{E}+00 & 2.4 \mathrm{E}+00\end{array}$

Oose Commitments (person-rem) from Airborne Pathways Total Body GI-LLI Thyroid Bone Liver Lung

$\begin{array}{lllllll}\text { Infant } & 3.7 \mathrm{E}-03 & 3.6 \mathrm{E}-03 & 5.4 \mathrm{E}-02 & 3.7 \mathrm{E}-03 & 3.7 \mathrm{E}-03 & 4.0 \mathrm{E}-03 \\ \text { Child } & 4.0 \mathrm{E}-02 & 4.0 \mathrm{E}-02 & 3.0 \mathrm{E}-01 & 4.1 \mathrm{E}-02 & 4.1 \mathrm{E}-02 & 4.5 \mathrm{E}-02 \\ \text { Teen } & 2.9 \mathrm{E}-02 & 2.9 \mathrm{E}-02 & 1.3 \mathrm{E}-01 & 2.9 \mathrm{E}-02 & 2.9 \mathrm{E}-02 & 3.6 \mathrm{E}-02 \\ \text { Adult } & 1.8 \mathrm{E}-01 & 1.8 \mathrm{E}-01 & 5.1 \mathrm{E}-01 & 1.8 \mathrm{E}-01 & 1.8 \mathrm{E}-01 & 2.0 \mathrm{E}-01 \\ \text { TOTAL } & 2.5 \mathrm{E}-01 & 2.5 \mathrm{E}-01 & 1.0 \mathrm{E}+00 & 2.5 \mathrm{E}-01 & 2.5 \mathrm{E}-01 & 2.8 \mathrm{E}-01\end{array}$

Production/Consumption factors:

Produce: 1 Milk: 1 Meat: 1

FRACTION DF POPULATION RECEIVING AN AVERAGE INDIVIDUAL

TOTAL-BOCY DGSE COMMITMENT FROM AIRBORNE PATHWAYS

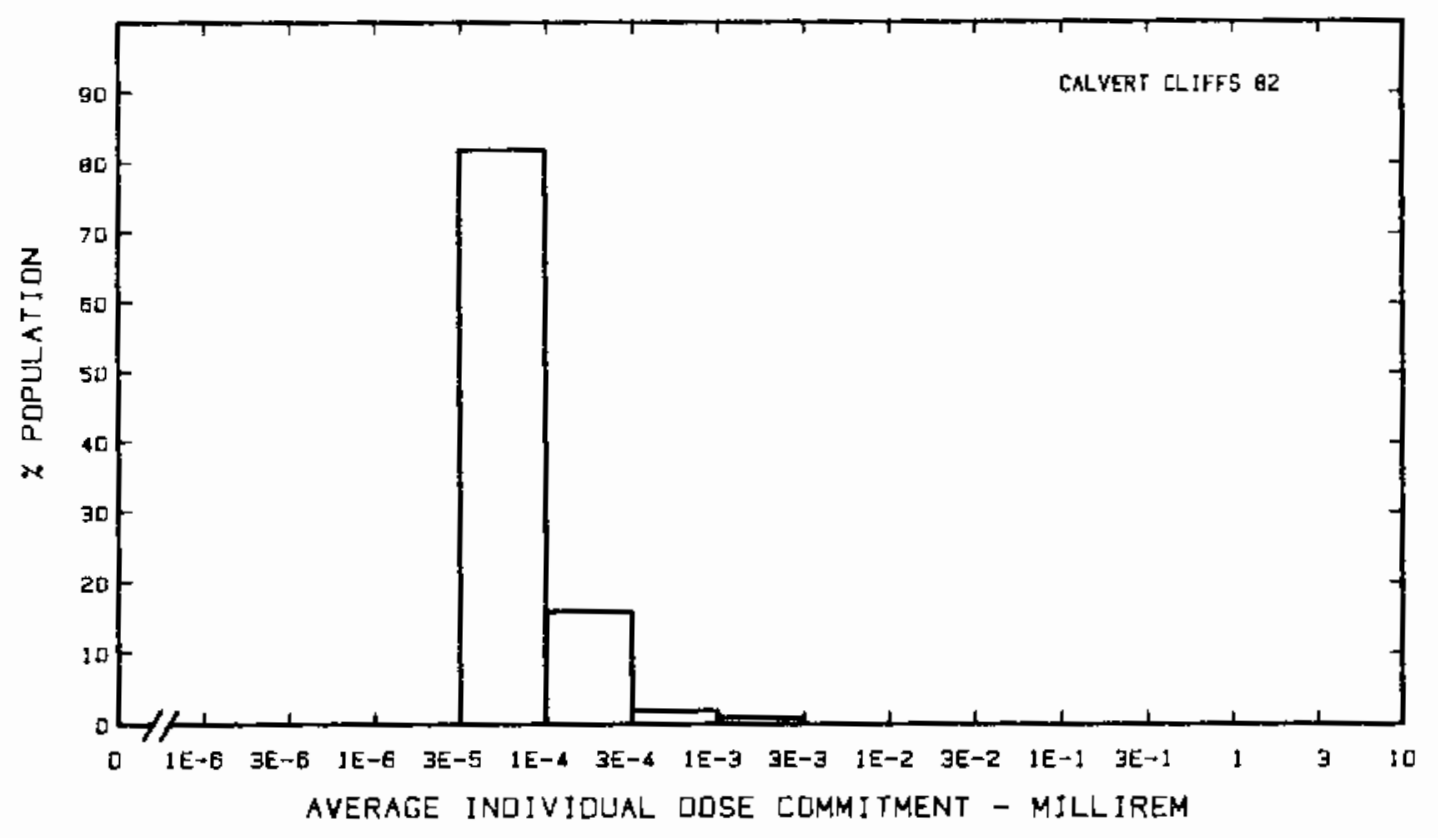


Site: $\operatorname{COOK}$

BENTON HARBOR, MICHIGAN

Location: $\quad$ N $41.9761^{\circ} \quad$ W $86.5664^{\circ}$

POPULATION DATA

Total Population Within 2-to-80-km Region: 1.1 E6

Major Metropolitan Centers Within Region:

\begin{tabular}{lcccc}
\multicolumn{1}{c}{ Center } & Population & \multicolumn{2}{c}{ Location } \\
\cline { 2 - 4 } & & & \\
Gary & 150,000 & $77 \mathrm{~km}$ & $\mathrm{SW}$ \\
South Bend SMSA & 280,000 & $42 \mathrm{~km}$ & SE \\
Elkhart SMSA & 140,000 & $58 \mathrm{~km}$ & SE \\
Mishiwaka & 40,000 & $47 \mathrm{~km}$ & SE \\
Michigan City & 37,000 & $40 \mathrm{~km}$ & SW
\end{tabular}

SITE SPECIFIC DATA - AIRBORNE PATHWAYS

Average Annual State Production

of Crops and Animal Products

In $80-\mathrm{km}$ Radius Circle

Regional Productivity Factor:

Animal Grazing Factor:
Veg: 1.1E8 kilogram

Milk: 2.3E8 liter

Meat: 1.9E8 kilogram

0.6

0.5

Meteorology Period of Record: 1 MAY 75 - 31 APR 76 Recovery: 95\%

SITE SPECIFIC DATA - WATERBORNE PATHWAYS via LAKE MICHIGAN

Average Dilution Flow

from Plant: $3,100 \mathrm{ft}^{3} / \mathrm{s}$

Orinking Water:

Exposed Population: $26 \rho_{a}$ Q00

Dilution Factur: 0.025

Fish:

Edible Harvest: $1.5 \mathrm{E} 6 \mathrm{~kg} / \mathrm{yr}$ Dilution Factor: 0.01

(a) Drinking water dilution factor estimated by averaging dilution factors derived from FES (1973) suitably weighted for population. 
POPULATION DOSE-COMMITMENT ESTIMATES AND

AVERAGE INDIVIDUAL DOSE-COMMITMENT HISTOGRAM FOR

COOK 1 AND 2

Dose Commitments (person-rem) from Liquid Pathways

\begin{tabular}{|c|c|c|c|c|c|}
\hline & Total Body & GI-LLI & Thyroid & Bone & Liver \\
\hline $\begin{array}{l}\text { Infant } \\
\text { Child } \\
\text { Teen } \\
\text { Adult }\end{array}$ & $\begin{array}{l}2.5 \mathrm{E}-03 \\
5 . \mathrm{DE}-02 \\
5.2 \mathrm{E}-02 \\
5.5 \mathrm{E}-01\end{array}$ & $\begin{array}{l}2.2 \mathrm{E}-03 \\
2.8 \mathrm{E}-02 \\
1.5 \mathrm{E}-02 \\
1.3 \mathrm{E}-01\end{array}$ & $\begin{array}{l}\text { 2. 3E-D2 } \\
1.7 \mathrm{E}-01 \\
5.5 \mathrm{E}-02 \\
4.0 \mathrm{E}-01\end{array}$ & $\begin{array}{l}1.8 \mathrm{E}-03 \\
1.2 \mathrm{E}-01 \\
6.8 \mathrm{E}-02 \\
4.0 \mathrm{E}-01\end{array}$ & $\begin{array}{l}\text { 4.2E-03 } \\
1.6 \mathrm{E}-01 \\
1.2 \mathrm{E}-01 \\
7.2 \mathrm{E}-01\end{array}$ \\
\hline TOTAL & $6.5 \mathrm{E}-01$ & $1.7 \mathrm{E}-01$ & $6.5 \mathrm{E}-01$ & $5.9 \mathrm{E}-01$ & $1.0 E+00$ \\
\hline
\end{tabular}

Dose Commitments (person-rem) from Airborne Pathways

Total Body GI-LLI Thyroid Bone Liver Lung

$\begin{array}{lllllll}\text { Infant } & 9.1 \mathrm{E}-04 & 7.2 \mathrm{E}-04 & 5.2 \mathrm{E}-02 & 2.0 \mathrm{E}-03 & 2.5 \mathrm{E}-03 & 9.4 \mathrm{E}-04 \\ \text { Child } & 1.1 \mathrm{E}-02 & 8.1 \mathrm{E}-03 & 3.5 \mathrm{E}-01 & 2.1 \mathrm{E}-02 & 2.3 \mathrm{E}-02 & 1.0 \mathrm{E}-02 \\ \text { Teen } & 8.5 \mathrm{E}-03 & 6.0 \mathrm{E}-03 & 1.4 \mathrm{E}-01 & 9.8 \mathrm{E}-03 & 1.2 \mathrm{E}-02 & 7.5 \mathrm{E}-03 \\ \text { Adult } & 5.0 \mathrm{E}-02 & 3.6 \mathrm{E}-02 & 4.8 \mathrm{E}-01 & 4.7 \mathrm{E}-02 & 5.6 \mathrm{E}-02 & 4.0 \mathrm{E}-02 \\ \text { TOTAL } & 7.1 \mathrm{E}-02 & 5.1 \mathrm{E}-02 & 1.0 \mathrm{E}+0 \mathrm{D} & 8 . \mathrm{DE}-02 & 9.4 \mathrm{E}-02 & 5.9 \mathrm{E}-02\end{array}$

Production/Consumption factors:

Produce: 1 Milk: 1 Meat: 1.2

FRACTION DF POPULATION RECEIVING AN AVERAGE INOIVIDUAL TOTAL-BODY DOSE COMMITMENT FROM AIRBORNE PATHWAYS

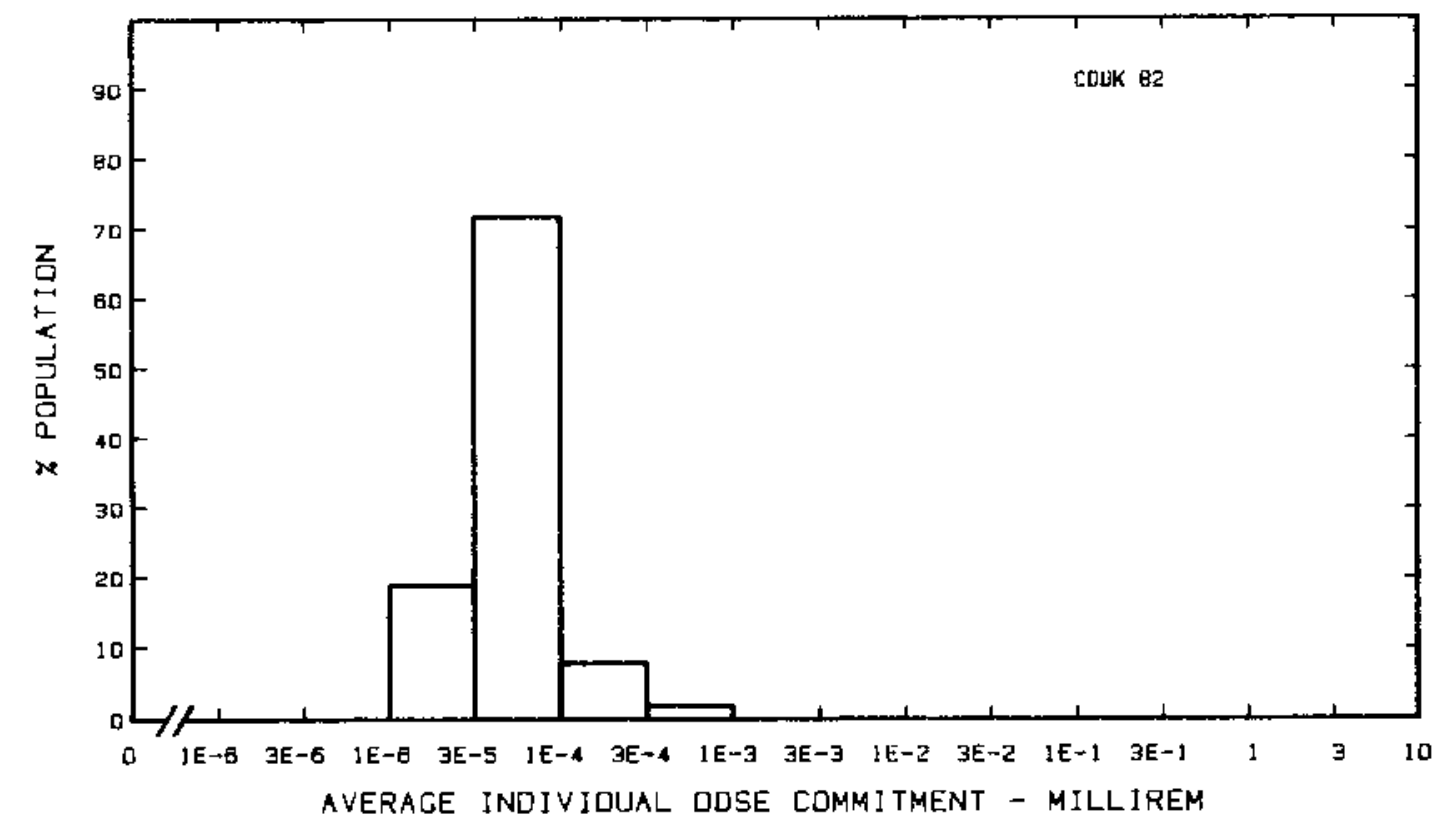


Site: COOPER

NEMAHA COUNTY, NEBRASKA

Location: N $40.3619^{\circ}$ W $95.6411^{\circ}$

POPULATION DATA

Total Population Within 2-to-80-km Region: 1.7E5

Major Metropolitan Centers Wi:hin Region:

Center
Nebraska City
Red Oak
Plattsmouth
Shenandoah
Falls City

Population

Location

7,100

6,800

6,300

6,300

5,400

$40 \mathrm{~km} \quad \mathrm{NNW}$

$80 \mathrm{~km} \quad$ NNE

$76 \mathrm{~km} \quad \mathrm{NNW}$

$51 \mathrm{~km} \quad \mathrm{NNE}$

$33 \mathrm{~km} \mathrm{~S}$

SITE SPECIFIC DATA - AIRBORNE PATHWAYS

Average Annual State Production

Veg: 9.7E7 kilogram

of Crops and Animal Products

In $80-\mathrm{km}$ Radius Circle

Milk: $7.2 E 7$ liter

Regional Productivity Factor:

Animal Grazing Factor:

Meat: $2.0 \mathrm{E} 8 \mathrm{kjlogram}$

Meteorology Period of Record: 1 MAR 70 - 31 DEC 75 Recovery: 89\%

SITE SPECIFIC DATA - WATERBORNE PATHWAYS via MISSOURI RIVER

Average River Flow

at Site: $31,000 \mathrm{ft}^{3} / \mathrm{s}$

Drinking Water:

Exposed Population: None

Fish:

Edible Harvest: $5.0 \mathrm{E}(\mathrm{a}) \mathrm{kg} / \mathrm{yr}$
Dilution Factor: $\mathrm{D.5}(\mathrm{a})$

(a) Assumes $1 / 2$ fish harvest caught below plant. 
POPULATION DOSE-COMMITMENT ESTIMATES AND
AVERAGE INDIVIDUAL DOSE-COMMITMENT HISTOGRAM FOR COOPER

Dose Commitments (person-rem) from Liquid Pathways Total Body GI-LLI Thyroid Bone Liver

$\begin{array}{llllll}\text { Infant } & 0.0 \mathrm{E}+\mathrm{D0} & 0.0 \mathrm{E}+00 & 0.0 \mathrm{E}+00 & 0.0 \mathrm{E}+00 & 0.0 \mathrm{E}+00 \\ \text { Child } & 1.1 \mathrm{E}-03 & 1.0 \mathrm{E}-04 & 3.5 \mathrm{E}-05 & 5.1 \mathrm{E}-03 & 6.2 \mathrm{E}-03 \\ \text { Teen } & 2.2 \mathrm{E}-03 & 2.1 \mathrm{E}-04 & 2.6 \mathrm{E}-05 & 3.1 \mathrm{E}-03 & 5.3 \mathrm{E}-03 \\ \text { Adult } & 2.3 \mathrm{E}-02 & 1.8 \mathrm{E}-03 & 1.7 \mathrm{E}-04 & 1.8 \mathrm{E}-02 & 3.2 \mathrm{E}-02 \\ \text { TOTAL } & 2.7 \mathrm{E}-02 & 2.1 \mathrm{E}-03 & 2.3 \mathrm{E}-04 & 2.6 \mathrm{E}-02 & 4.3 \mathrm{E}-02\end{array}$

Dose Commitments (person-rem) from Airborne Pathways Total Body GI-LLI Thyroid Bone Liver Lung

$\begin{array}{lllllll}\text { Infant } & 2.0 \mathrm{E}-03 & 2.0 \mathrm{E}-03 & 1.6 \mathrm{E}-02 & 2.1 \mathrm{E}-03 & 2.1 \mathrm{E}-03 & 2.1 \mathrm{E}-03 \\ \text { Child } & 2.3 \mathrm{E}-02 & 2.2 \mathrm{E}-02 & 1.6 \mathrm{E}-01 & 2.5 \mathrm{E}-02 & 2.3 \mathrm{E}-02 & 2.3 \mathrm{E}-02 \\ \text { Teen } & 1.7 \mathrm{E}-02 & 1.6 \mathrm{E}-02 & 6.7 \mathrm{E}-02 & 1.7 \mathrm{E}-02 & 1.7 \mathrm{E}-02 & 1.7 \mathrm{E}-02 \\ \text { Adult } & 9.9 \mathrm{E}-02 & 9.9 \mathrm{E}-02 & 2.8 \mathrm{E}-01 & 1.0 \mathrm{E}-01 & 9.9 \mathrm{E}-02 & 1.0 \mathrm{E}-01 \\ \text { TOTAL } & 1.4 \mathrm{E}-01 & 1.4 \mathrm{E}-01 & 5.2 \mathrm{E}-01 & 1.5 \mathrm{E}-01 & 1.4 \mathrm{E}-01 & 1.4 \mathrm{E}-01\end{array}$

Production/Consumption factors:
Produce: 2.9
Milk: 3.2
Meat: 14

FRACTION OF POPULATION RECEIVING AN AVERAGE INDIVIDUAL TOTAL-BODY DOSE COMMITMENT FROM AIRBORNE PATHWAYS

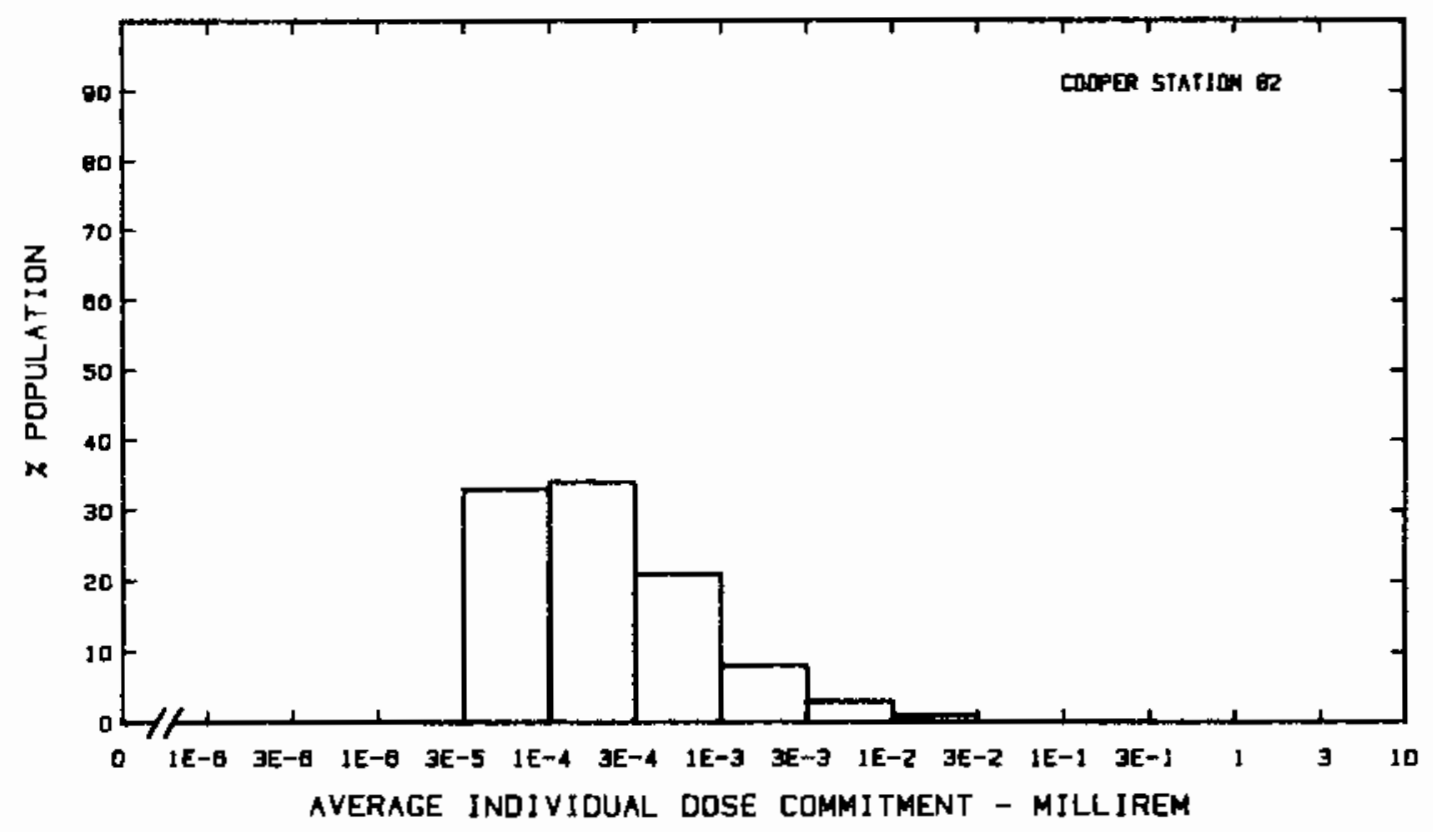


Site: CRYSTAL RIVER

CRYSTA- RIVER, FLORIDA

Location: $\quad N 28.9572^{\circ} \quad$ W $82.6939^{\circ}$

POPULATION DATA

Total Population Within 2-to-80-km Region: 3.8E5

Major Metropolitan Centers Within Region:

Center Population Location

$\begin{array}{llll}\text { Ocala } & 38,00 \mathrm{D} & 60 \mathrm{~km} & \text { ENE }\end{array}$

$\begin{array}{llll}\text { Leesburg } & 13,000 & 80 \mathrm{~km} & \mathrm{E} \\ \text { New Port Richey } & 11,000 & 79 \mathrm{~km} & \mathrm{~S}\end{array}$

SITE SPECIFIC OATA - AIRBORNE PATHWAYS

Average Annual State Production Veg: 2.8E7 kilogram

of Crops and Animal Products Milk: 1.1E8 liter

In 80-km Radius Circle Meat: 7.2E7 kilogram

Regional Productivity Factor: $\quad 0.5$

Animal Grazing Factor:

1

Meteorology Period of Record: 1 JAN 75 - 3l DEC 75 Recovery: 93\%

SITE SPECIFIC DATA - WATERBORNE PATHWAYS via GULF Of MEXICO

Average Dilution Flow

from Plant: $1,400 \mathrm{ft}^{3} / \mathrm{s}$

Fish: Edible Harvest: $3.2 \mathrm{E} 5 \mathrm{~kg} / \mathrm{yr}$

Dilution Factor: 0.1

Invertebrates: $\quad$ Edible Harvest: $1.8 \mathrm{E} 5 \mathrm{~kg} / \mathrm{yr}$

Dilution Factor: 0.1 
POPULATION DOSE-COMMITMENT ESTIMATES AND AVERAGE INDIVIDUAL DOSE-COMMITMENT HISTOGRAM FOR CRYSTAL RIVER 3

Dose Commitments (person-rem) from Liquid Pathways

\begin{tabular}{lcccccc} 
& Total Body & GI-LLI & & Thyroid & Bone & Liver \\
\cline { 2 - 3 } Infant & $0.0 \mathrm{E}+00$ & $0.0 \mathrm{E}+00$ & & $0.0 \mathrm{E}+00$ & $0.0 \mathrm{E}+00$ & $0.0 \mathrm{E}+00$ \\
Child & $6.3 \mathrm{E}-04$ & $1.9 \mathrm{E}-03$ & $3.2 \mathrm{E}-03$ & $6.5 \mathrm{E}-04$ & $9.1 \mathrm{E}-04$ \\
Teen & $5.7 \mathrm{E}-04$ & $3.8 \mathrm{E}-03$ & $2.3 \mathrm{E}-03$ & $4.0 \mathrm{E}-04$ & $7.8 \mathrm{E}-04$ \\
Adult & $4.5 \mathrm{E}-03$ & $3.3 \mathrm{E}-02$ & $1.5 \mathrm{E}-02$ & $2.4 \mathrm{E}-03$ & $4.8 \mathrm{E}-03$ \\
TOTAL & $5.8 \mathrm{E}-03$ & $3.9 \mathrm{E}-02$ & $2.1 \mathrm{E}-02$ & $3.4 \mathrm{E}-03$ & $6.5 \mathrm{E}-03$
\end{tabular}

Dose Commitments (person-rem) from Airborne Pathways

Total Body GI-LLI Thyroid Bone Liver Lung

$\begin{array}{lllllll}\text { Infant } & 3.5 \mathrm{E}-04 & 3.4 \mathrm{E}-04 & 7.6 \mathrm{E}-04 & 3.8 \mathrm{E}-04 & 4.3 \mathrm{E}-04 & 3.8 \mathrm{E}-04 \\ \text { Child } & 4.0 \mathrm{E}-03 & 3.9 \mathrm{E}-03 & 6.3 \mathrm{E}-03 & 4.1 \mathrm{E}-03 & 4.5 \mathrm{E}-03 & 4.3 \mathrm{E}-03 \\ \text { Teen } & 2.9 \mathrm{E}-03 & 2.8 \mathrm{E}-03 & 3.8 \mathrm{E}-03 & 2.8 \mathrm{E}-03 & 3.1 \mathrm{E}-03 & 3.3 \mathrm{E}-03 \\ \text { Adult } & 1.7 \mathrm{E}-02 & 1.7 \mathrm{E}-02 & 2.0 \mathrm{E}-02 & 1.6 \mathrm{E}-02 & 1.8 \mathrm{E}-\mathrm{D2} & 1.8 \mathrm{E}-02 \\ \text { TOTAL } & 2.5 \mathrm{E}-02 & 2.4 \mathrm{E}-02 & 3.1 \mathrm{E}-02 & 2.4 \mathrm{E}-02 & 2.6 \mathrm{E}-\mathrm{D} 2 & 2.6 \mathrm{E}-02\end{array}$

Production/Consumption factors:

Produce: <1 Milk: $1.2 \quad$ Meat: 1.2

FRACTION OF POPULATION RECEIVING AN AVERAGE INDIVIDUAL TDTAL-BODY DOSE COMMITMENT FROM AIRBORNE PATHWAYS

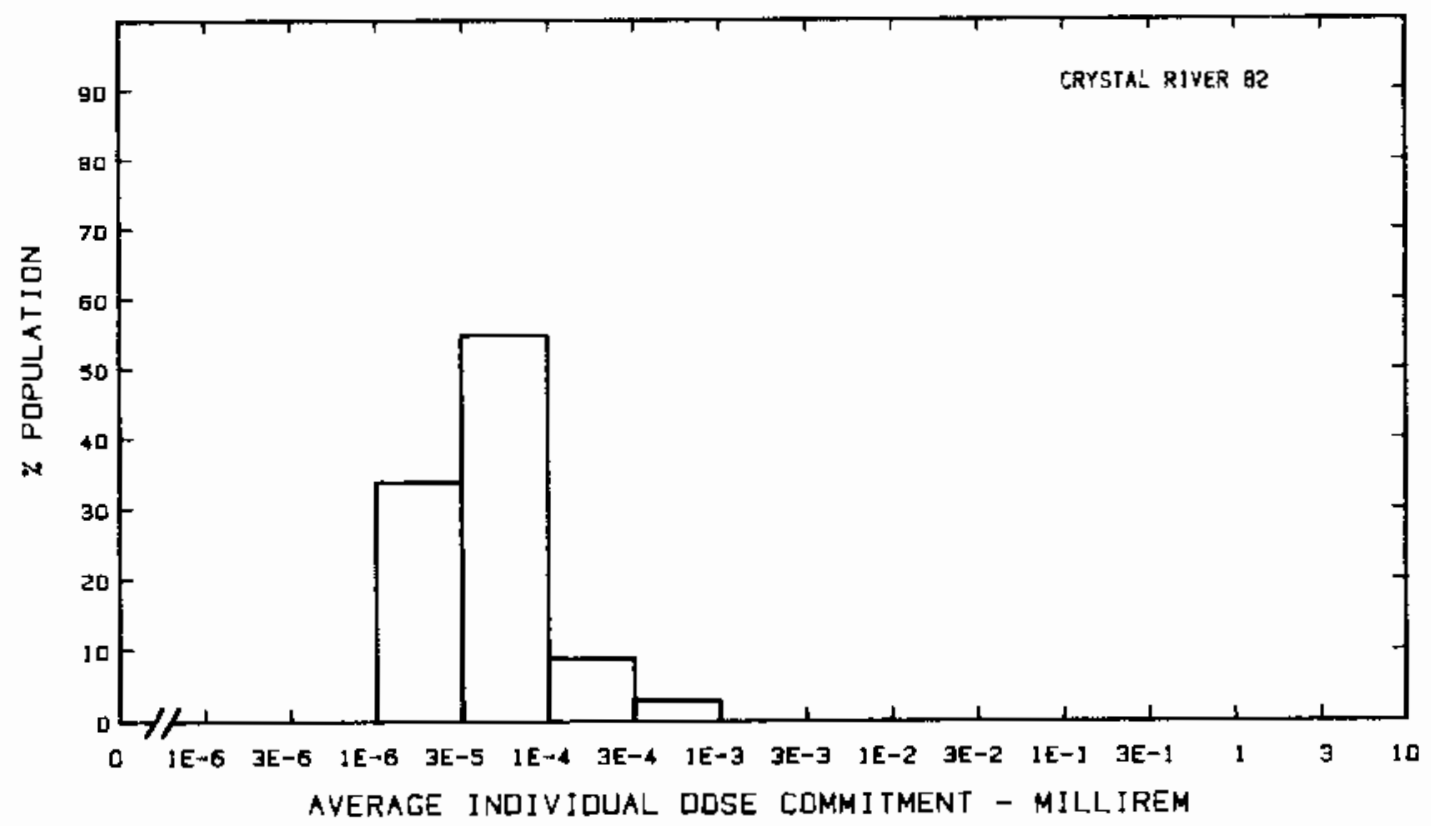


Site: DAVIS-BESSE

PORT CLINTON, OHIO

Location: $\quad N 41.5972^{\circ} \quad$ W $83.0864^{\circ}$

POPULATION DATA

Total Population Within 2-to-80-km Region: 1.8E6

Major Metropolitan Centers Within Region:

Center
Toledo SMSA
Dearborn
Taylor
Lorain
Lincoln Park
Findlay
Sandusky

Population

Location

$38 \mathrm{~km} \quad$ WNW

$80 \mathrm{~km} \quad \mathrm{~N}$

$71 \mathrm{~km} \mathrm{~N}$

$77 \mathrm{~km} \quad \mathrm{ESE}$

$73 \mathrm{~km} \mathrm{~N}$

$77 \mathrm{~km} \quad \mathrm{SW}$

$35 \mathrm{~km} \quad$ ESE

SITE SPECIFIC DATA - AIRBORNE PATHWAYS

Average Annual State Production

of Crops and Animal Products

In 80-km Radius Circle

Regional Productivity Factor:

Animal Grazing Factor:
Veg: $6.9 E 7$ kilogram

Milk: $3.7 E 8$ liter

Meat: 1.2E8 kilogram

0.5

0.5

Meteorology Period of Record: 4 AUG 74 - 3 AUG 76 Recovery: 99\%

SITE SPECIFIC DATA - WATERBORNE PATHWAYS via LAKE ERIE

Average Dilution Flow

from Plant: $37 \mathrm{ft}^{3} / \mathrm{s}$

Drinking Water:

Exposed Population: 450,000

Dilution Factor: 2.0E-4

Fish:

Edible Harvest: $5.7 \mathrm{E}^{(\mathrm{b})} \mathrm{kg} / \mathrm{yr}$

Dilution Factor: $1.8 \mathrm{E}-4^{(\mathrm{b})}$

(a) Drinking water dilution factor estimated by averaging dilution factor derived from FES (1973), suitably weighted for population.

(b) Letter from Terry D. Murray, Toledo Edison Company to Charles A. Willis, NRC, July 20, 1984. 
POPULATION DOSE-COMMITMENT ESTIMATES AND

AVERAGE INDIVIDUAL DOSE-COMMITMENT HISTOGRAM FOR

\section{DAVIS-BESSE}

Dose Comunitments (person-rem) from Liquid Pathways

Total Body GI-LLI Thyroid Bone Liver

$\begin{array}{llllll}\text { Infant } & 1.0 \mathrm{E}-04 & 1.1 \mathrm{E}-04 & 1.9 \mathrm{E}-04 & 3.4 \mathrm{E}-05 & 1.4 \mathrm{E}-04 \\ \text { Child } & 3.8 \mathrm{E}-03 & 1.7 \mathrm{E}-03 & 1.9 \mathrm{E}-03 & 1.4 \mathrm{E}-02 & 1.7 \mathrm{E}-02 \\ \text { Teen } & 5.5 \mathrm{E}-03 & 1.4 \mathrm{E}-03 & 7.2 \mathrm{E}-04 & 8.3 \mathrm{E}-03 & 1.3 \mathrm{E}-02 \\ \text { Adult } & 5.8 \mathrm{E}-02 & 1.2 \mathrm{E}-02 & 5.7 \mathrm{E}-03 & 4.8 \mathrm{E}-02 & 8.1 \mathrm{E}-02 \\ \text { TOTAL } & 6.8 \mathrm{E}-02 & 1.5 \mathrm{E}-02 & 8.5 \mathrm{E}-03 & 7.1 \mathrm{E}-02 & 1.1 \mathrm{E}-0 \mathrm{I}\end{array}$

Dose Commitments (person-rem) from Airborne Pathways

Total Body GI-LLI Thyroid Bone Liver Lung

$\begin{array}{lllllll}\text { Infant } & 2.7 \mathrm{E}-04 & 2.7 \mathrm{E}-04 & 5.1 \mathrm{E}-03 & 1.3 \mathrm{E}-04 & 2.8 \mathrm{E}-04 & 2.8 \mathrm{E}-04 \\ \text { Child } & 3.3 \mathrm{E}-03 & 3.2 \mathrm{E}-03 & 3.0 \mathrm{E}-02 & 1.5 \mathrm{E}-03 & 3.3 \mathrm{E}-03 & 3.4 \mathrm{E}-03 \\ \text { Teen } & 2.2 \mathrm{E}-03 & 2.1 \mathrm{E}-03 & 1.2 \mathrm{E}-02 & 1.0 \mathrm{E}-03 & 2.1 \mathrm{E}-03 & 2.3 \mathrm{E}-03 \\ \text { Adult } & 1.2 \mathrm{E}-02 & 1.2 \mathrm{E}-02 & 4.4 \mathrm{E}-02 & 6.0 \mathrm{E}-03 & 1.2 \mathrm{E}-02 & 1.2 \mathrm{E}-02 \\ \text { TOTAL } & 1.8 \mathrm{E}-02 & 1.7 \mathrm{E}-02 & 9.2 \mathrm{E}-02 & 8.7 \mathrm{E}-03 & 1.8 \mathrm{E}-02 & 1.8 \mathrm{E}-02\end{array}$

Production/Consumption factors:

Produce: $<1 \quad$ Milk: $<1 \quad$ Meat: $<1$

FRACTION OF POPULATION RECEIVING AN AVERAGE INOIVIDUAL TOTAL-EOOY DOSE COMMITMENT FROM AIRBORNE PATHWAYS

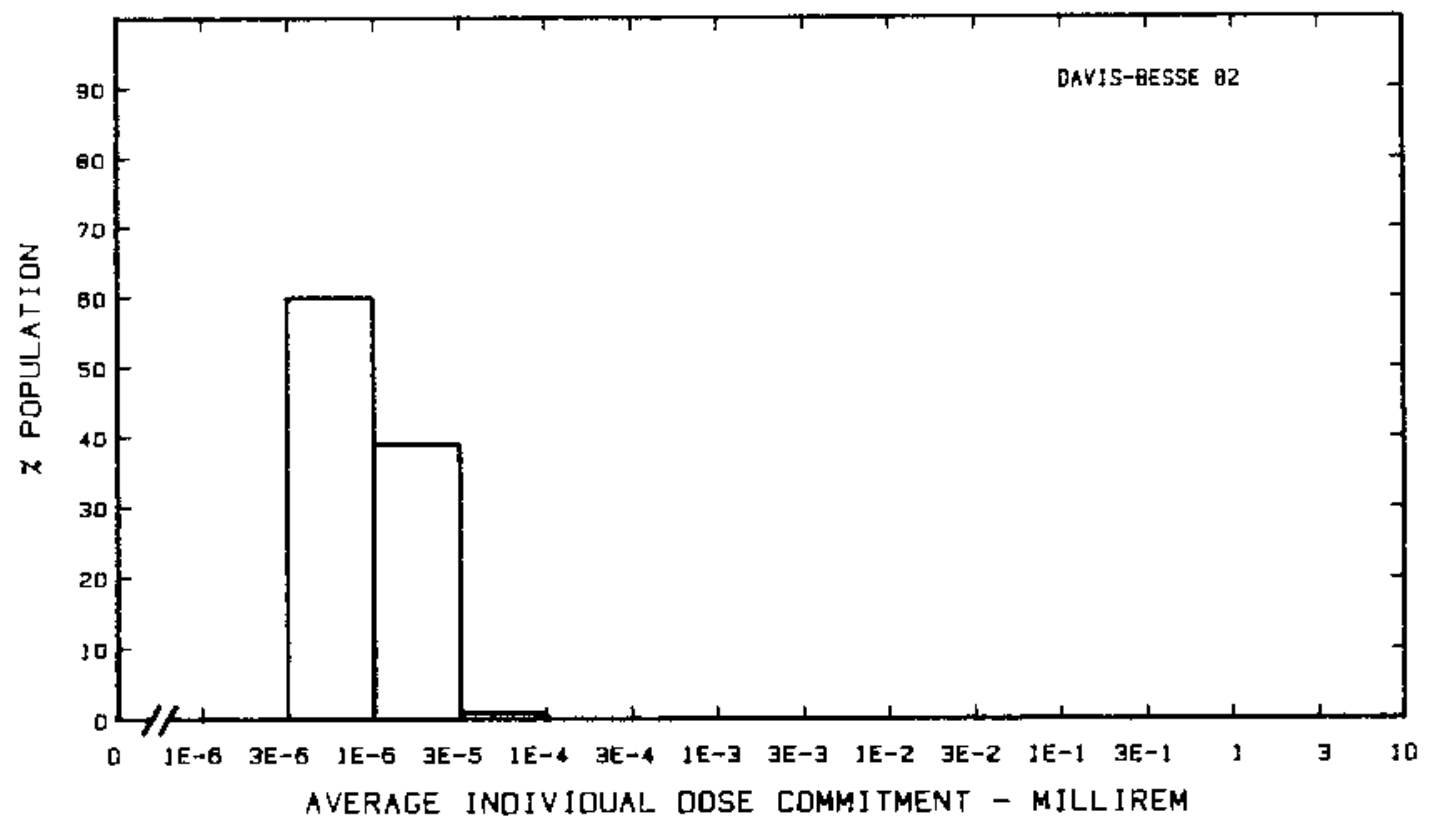


Site: DRESDEN

GRUNDY COUNTY, ILLINOIS

Location: $\quad$ N $41.3897^{\circ} \quad$ W $88.271 .1^{\circ}$

POPULATION DATA

Total Population Within 2-to-80-km Region: 6.346

Major Metropolitan Centers Within Region:

\begin{tabular}{|c|c|c|c|}
\hline Center & Population & Locat & \\
\hline $\begin{array}{l}\text { Chicago SMSA } \\
\text { Gary-Hammond-E.Chicago-SMSA } \\
\text { Kankakee SMSA } \\
\text { Aurora } \\
\text { Joliet } \\
\text { Elgin }\end{array}$ & $\begin{array}{c}7,100,000(a) \\
640,000(a) \\
100,000 \\
81,000 \\
78,000 \\
56,000\end{array}$ & $\begin{array}{ll}75 & \mathrm{~km} \\
80 & \mathrm{~km} \\
45 & \mathrm{~km} \\
41 & \mathrm{~km} \\
22 & \mathrm{~km} \\
74 & \mathrm{~km}\end{array}$ & $\begin{array}{l}\mathrm{NE} \\
\mathrm{ENE} \\
\mathrm{SE} \\
\mathrm{N} \\
\mathrm{NE} \\
\mathrm{N}\end{array}$ \\
\hline
\end{tabular}

SITE SPECIFIC DATA - AIRBORNE PATHWAYS

Average Annual State Production

of Crops and Animal Products

In 80-km Radius Circle

Regional Productivity Factor:

Animal Grazing Factor:

Meteorology Period of Record: 1 JAN 74 - 31 JAN 75 Recovery: 77\%
Veg: $1.1 \mathrm{E8} \mathrm{kilogram}$

Milk: $1.8 \mathrm{E} 8$ liter

Meat: 1.9E8 kilogram

1

0.5

SITE SPECIFIC DATA - WATERBORNE PATHWAYS via ILLINOIS RIVER

Average River Flow

at Site: $12,000 \mathrm{ft}^{3} / \mathrm{s}$

Drinking Water:

Exposed Population:

(b)

Fish:

Edible Harvest: (b) $\mathrm{kg} / \mathrm{yr}$

(a) Population of total SMSA given; population of SMSA fraction within $80 \mathrm{~km}$ of site would be somewhat smaller.

(b) River water used for sewage disposal for Chicago, so population doses from liquid pathways assumed to be near zero. 
POPULATION DOSE-COMMITMENT ESTIMATES AND

AVERAGE INDIVIDUAL DOSE-COMMITMENT HISTOGRAM FOR

DRESDEN 1,2 AND 3

Dose Commitments (person-rem) from Liquid Pathways

Total Body GI-LLI Ihyroid Bone Liver

Infant

Child

Teen

Adult

(Little or no Liquid Pathway Doses)

TOTAL

Dose Commitments (person-rem) from Airborne Pathways

Total Body GI-LLI Thyroid Bone Liver Lung

$\begin{array}{lllllll}\text { Infant } & 8.8 \mathrm{E}-02 & 8.7 \mathrm{E}-02 & 5.8 \mathrm{E}-01 & 8.6 \mathrm{E}-02 & 8.9 \mathrm{E}-02 & 9.6 \mathrm{E}-02 \\ \text { Child } & 1.0 \mathrm{E}+00 & 1.0 \mathrm{E}+00 & 5.5 \mathrm{E}+00 & 9.6 \mathrm{E}-\mathrm{D1} & 1.0 \mathrm{E}+00 & 1.1 \mathrm{E}+00 \\ \text { Teen } & 7.3 \mathrm{E}-01 & 7.4 \mathrm{E}-01 & 3.0 \mathrm{E}+00 & 6.9 \mathrm{E}-01 & 7.4 \mathrm{E}-01 & 8.5 \mathrm{E}-01 \\ \text { Adult } & 4.4 \mathrm{E}+00 & 4.5 \mathrm{E}+00 & 1.4 \mathrm{E}+01 & 4.2 \mathrm{E}+00 & 4.4 \mathrm{E}+00 & 4.8 \mathrm{E}+00 \\ \text { TOTAL } & 6.2 \mathrm{E}+00 & 6.3 \mathrm{E}+00 & 2.3 \mathrm{E}+01 & 5.9 \mathrm{E}+00 & 6.3 \mathrm{E}+00 & 6.9 \mathrm{E}+00\end{array}$

Production/Consumption factors:

Produce: $<1 \quad$ Milk: $<1 \quad$ Meat: $<1$

FRACTION OF POPULATION RECEIVING AN AVERAGE INDIVIDUAL TOTAL-BODY DOSE COMMITMENT FROM AIRBORNE PATHWAYS

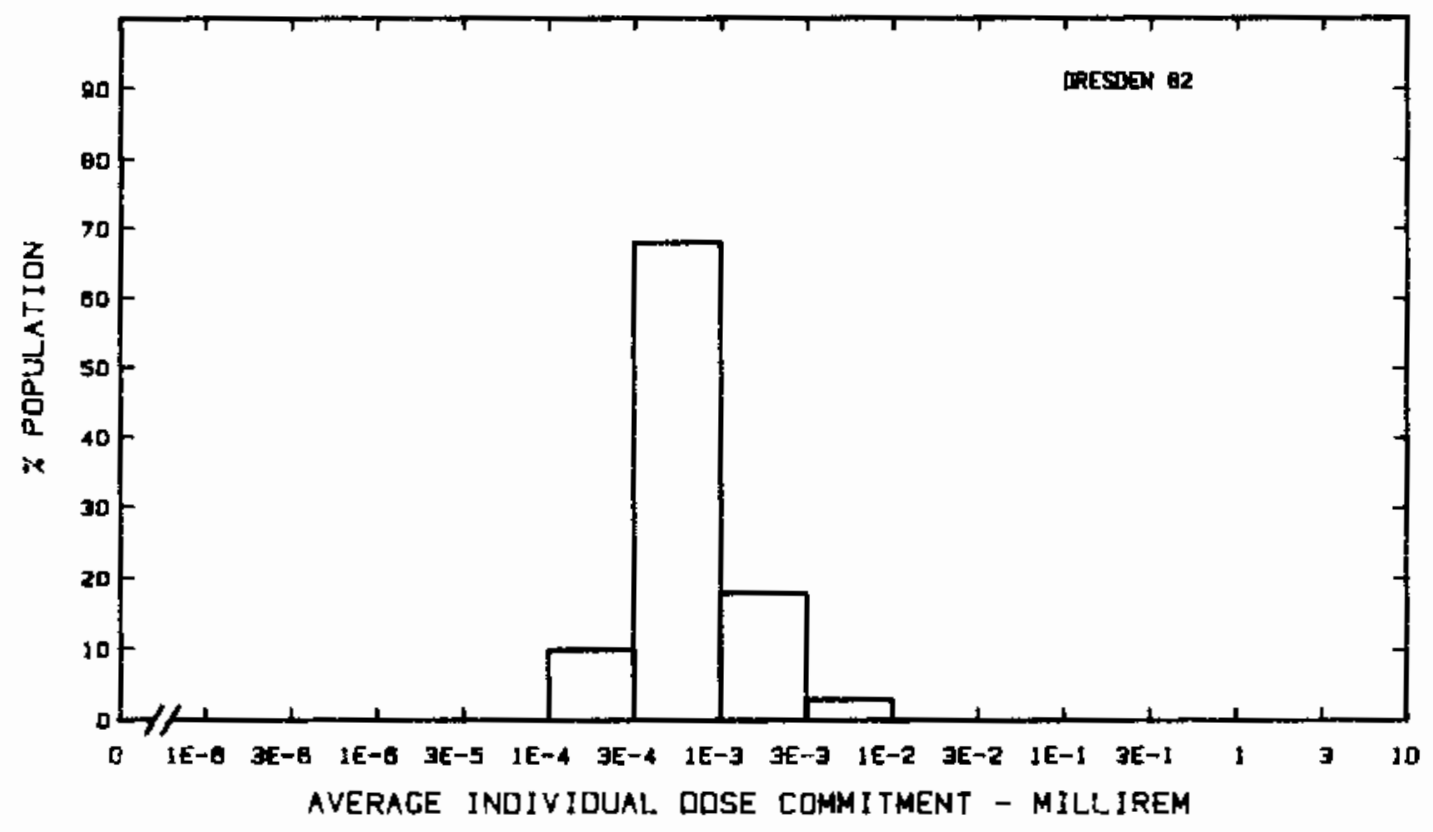


Site: DUANE ARNOLD

CEDAR RAPIDS, IOWA

Location: N $42.1006^{\circ} \quad$ W $91.7772^{\circ}$

POPULATION DATA

Total Population Within 2-to-80-km Region: 6.0E5

Major Metropolitan Centers Within Region:

\begin{tabular}{lrlll}
\multicolumn{2}{c}{ Center } & Population & \multicolumn{2}{l}{ Location } \\
\cline { 3 - 5 } Cedar Rapids SMSA & 170,000 & & $17 \mathrm{~km}$ & SE \\
Waterloo-Cedar Falls SMSA & 140,000 & & $64 \mathrm{~km}$ & NW \\
Iowa City SMSA & 82,000 & $52 \mathrm{~km}$ & SSE \\
Marion & 19,000 & $16 \mathrm{~km}$ & ESE
\end{tabular}

SITE SPECIFIC DATA - AIRBORNE PATHWAYS

Average Annual State Production

of Crops and Animal Products

In 80-km Radius Circle

Veg: $9.8 \mathrm{E} 7 \mathrm{ki}$ logram

Milk: 2.6E8 liter

Meat: $4.2 \mathrm{E} 8 \mathrm{kilogram}$

Regional Productivity Factor:

Animal Grazing Factor:

1

0.5

Meteorology Period of Record: 1 FEB 71 - 31 DEC 75 Recovery: 57\%

SITE SPECIFIC DATA - WATERBORNE PATHWAYS via CEDAR RIVER

Average River Flow

at Site: $3,100 \mathrm{ft}^{3} / \mathrm{s}$

Drinking Water:

Exposed Population: 110,000

Dilution Factor: 1

Fish:

Edible Harrest: (a)

Dilution Factor: 1

(a) No fish catch data given in FES (1973); thus, population was assumed to eat fish at generic consumption rates (Table A.1). 
POPULATION DOSE-COMMITMENT ESTIMATES AND

AVERAGE INDIVIDUAL DOSE-CDMMITMENT HISTOGRAM FOR

DUANE ARNOLD

Dose Commitments (person-rem) from Liquid Pathways

\begin{tabular}{|c|c|c|c|c|c|}
\hline & Tota 1 Body & GI-LLI & Thyroid & Bone & Liver \\
\hline $\begin{array}{l}\text { Infant } \\
\text { Child } \\
\text { Teen } \\
\text { Adult }\end{array}$ & $\begin{array}{l}0.0 \mathrm{E}+00 \\
1.3 \mathrm{E}-08 \\
9.8 \mathrm{E}-09 \\
6.3 \mathrm{E}-08\end{array}$ & $\begin{array}{l}0 . \mathrm{DE}+00 \\
2.0 \mathrm{E}-08 \\
4.4 \mathrm{E}-08 \\
4.0 \mathrm{E}-07\end{array}$ & $\begin{array}{l}0.0 \mathrm{E}+00 \\
3.1 \mathrm{E}-10 \\
2.8 \mathrm{E}-10 \\
2.3 \mathrm{E}-09\end{array}$ & $\begin{array}{l}0.0 \mathrm{E}+00 \\
1.6 \mathrm{E}-08 \\
1.3 \mathrm{E}-08 \\
9.4 \mathrm{E}-08\end{array}$ & $\begin{array}{l}0.0 \mathrm{E}+00 \\
9.4 \mathrm{E}-09 \\
8.8 \mathrm{E}-09 \\
5.5 \mathrm{E}-08\end{array}$ \\
\hline Total & $8.6 \mathrm{E}-08$ & $4.6 \mathrm{E}-07$ & $2.8 \mathrm{E}-09$ & $1.2 \mathrm{E}-07$ & 7. $3 \mathrm{E}-08$ \\
\hline
\end{tabular}

Oose Commitments (person-rem) from Airborne Pathways

Total Body GI-LLI Thyroid Bone Liver Lung

$\begin{array}{lllllll}\text { Infant } & 9.4 \mathrm{E}-05 & 9.0 \mathrm{E}-05 & 1.9 \mathrm{E}-03 & 9.7 \mathrm{E}-05 & 9.6 \mathrm{E}-05 & 9.5 \mathrm{E}-05 \\ \text { Child } & 1.4 \mathrm{E}-03 & 1.2 \mathrm{E}-03 & 1.9 \mathrm{E}-02 & 2.4 \mathrm{E}-03 & 1.2 \mathrm{E}-03 & 1.2 \mathrm{E}-03 \\ \text { Teen } & 9.1 \mathrm{E}-04 & 8.2 \mathrm{E}-04 & 7.9 \mathrm{E}-03 & 1.3 \mathrm{E}-03 & 8.1 \mathrm{E}-04 & 8.6 \mathrm{E}-04 \\ \text { Adult } & 5.2 \mathrm{E}-03 & 4.9 \mathrm{E}-03 & 3.1 \mathrm{E}-02 & 6.6 \mathrm{E}-03 & 4.7 \mathrm{E}-03 & 4.9 \mathrm{E}-03 \\ \text { TOTAL } & 7.6 \mathrm{E}-03 & 6.9 \mathrm{E}-03 & 6.0 \mathrm{E}-02 & 1.0 \mathrm{E}-02 & 6.8 \mathrm{E}-03 & 7.1 \mathrm{E}-03\end{array}$

Production/Consumption factors:

Produce: <1 Milk: $3.3 \quad$ Meat: 8.7

FRACTION DF POPLLATION RECEIVING AN AVERAGE INDIVIDUAL TOTAL-BODY DOSE COMHITMENT FRDM AIREORNE PATHWAYS

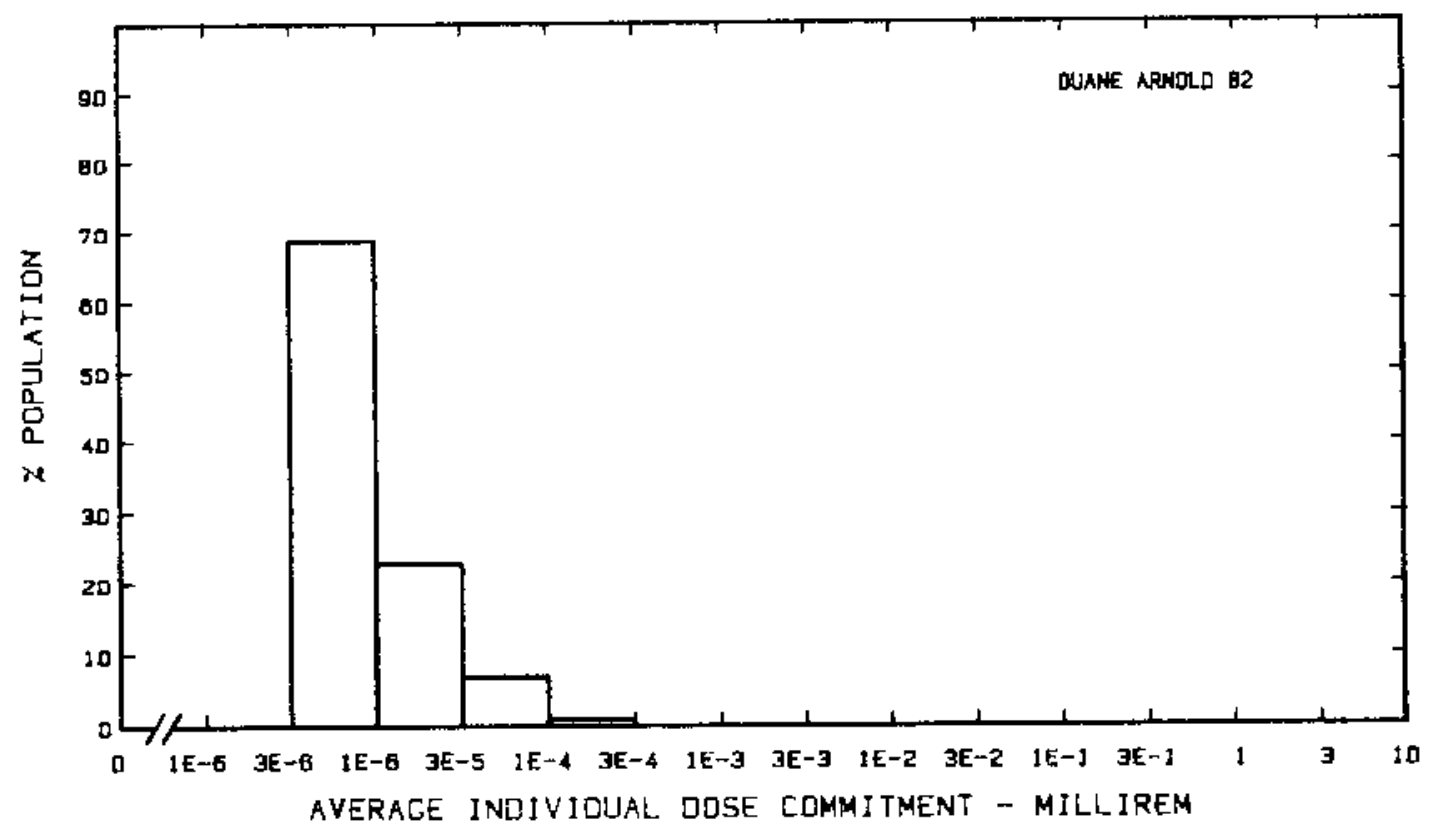


Site: J. M. FARLEY

DOTHAN, ALABAMA

Location: $\quad \mathrm{N} 31.2228^{\circ}$ W $85.1126^{\circ}$

POPULATION DATA

Total Population Within 2-to-80-km Region: 3.6E5

Major Metropolitan Centers Within Region:

Center
Dothan
Enterprise
Ozark
Eufaula
Bainbridge

Population Location

Dothan

49,000

18,000

13,000

12,000

11,000

$27 \mathrm{~km}$

$71 \mathrm{~km}$

$56 \mathrm{~km}$

$75 \mathrm{~km} \quad \mathrm{~N}$

Bainbridge

$$
11,000
$$

$62 \mathrm{~km} \quad \mathrm{SE}$

SITE SPECIFIC DATA - AIRBORNE PATHWAYS

Average Annual State Production

Of Crops and Animal Products

In 80-km Radius Circle

Regional Productivity Factor:

Animal Grazing Factor:

Meteorology Period of Record: 1 APR 71 - 31 MAR 75 Recovery: 100\%
Veg: $1.7 \mathrm{E} 7 \mathrm{ki} \log \mathrm{ram}$

Milk: 5.7 E7 liter

Meat: $8.6 E 7$ kilogram

0.95

0.8

SITE SPECIFIC DATA - WATERBORNE PATHWAYS via CHATTAHOOCHEE RIVER

Average River Flow at Site: $12,000 \mathrm{ft}^{3} / \mathrm{s}$

Orinking Water:

Exposed Population: None

Fish:

Edible Harvest: $2.3 \mathrm{E} 5 \mathrm{~kg} / \mathrm{yr}$

Dilution Factor: 1 


\section{POPULATION DOSE-COMMITMENT ESTIMATES AND}

AVERAGE INDIVIOUAL DOSE-COMMITMENT HISTOGRAM FOR

J. M. FARLEY 1 ANO 2

Dose Commitments (person-rem) from Liquid Pathways

\begin{tabular}{|c|c|c|c|c|c|}
\hline & Total Body & GI-LLI & Thyroid & Bone & Liver \\
\hline $\begin{array}{l}\text { Infant } \\
\text { Child } \\
\text { Teen } \\
\text { Adult }\end{array}$ & $\begin{array}{l}0.0 \mathrm{E}+00 \\
1.9 \mathrm{E}-03 \\
3.5 \mathrm{E}-03 \\
3.8 \mathrm{E}-03\end{array}$ & $\begin{array}{l}0.0 \mathrm{E}+00 \\
5.7 \mathrm{E}-04 \\
1.0 \mathrm{E}-03 \\
8.5 \mathrm{E}-03\end{array}$ & $\begin{array}{l}0.0 \mathrm{E}+00 \\
2.7 \mathrm{E}-04 \\
2.2 \mathrm{E}-04 \\
1.7 \mathrm{E}-04\end{array}$ & $\begin{array}{l}0.0 E+00 \\
8.8 E-03 \\
5.3 E-03 \\
3.1 E-02\end{array}$ & $\begin{array}{l}0.0 \mathrm{E}+00 \\
1.0 \mathrm{E}-02 \\
8.7 \mathrm{E}-03 \\
5.2 \mathrm{E}-02\end{array}$ \\
\hline TOTAL & 4. $3 \mathrm{E}-02$ & 1.DE-02 & 2. $2 \mathrm{E}-03$ & 4. $5 \mathrm{E}-02$ & $7.1 \mathrm{E}-02$ \\
\hline
\end{tabular}

Dose Commitments (person-rem) from Airborne Pathways Total Body GI-LLI Thyroid Bone Liver Lung

$\begin{array}{lllllll}\text { Infant } & 2.1 \mathrm{E}-02 & 2.1 \mathrm{E}-02 & 5.8 \mathrm{E}-02 & 2.1 \mathrm{E}-02 & 2.1 \mathrm{E}-02 & 2.2 \mathrm{E}-\mathrm{D} 2 \\ \text { Child } & 2.4 \mathrm{E}-01 & 2.4 \mathrm{E}-01 & 5.2 \mathrm{E}-01 & 2.3 \mathrm{E}-01 & 2.4 \mathrm{E}-01 & 2.5 \mathrm{E}-01 \\ \text { Teen } & 1.7 \mathrm{E}-01 & 1.7 \mathrm{E}-01 & 3.1 \mathrm{E}-01 & 1.7 \mathrm{E}-01 & 1.7 \mathrm{E}-01 & 1.9 \mathrm{E}-01 \\ \text { Adult } & 1.1 \mathrm{E}+00 & 1.1 \mathrm{E}+00 & 1.6 \mathrm{E}+00 & 1.0 \mathrm{E}+00 & 1.1 \mathrm{E}+00 & 1.1 \mathrm{E}+00 \\ \text { ToTAL } & 1.5 \mathrm{E}+00 & 1.5 \mathrm{E}+00 & 2.5 \mathrm{E}+00 & 1.4 \mathrm{E}+00 & 1.5 \mathrm{E}+00 & 1.6 \mathrm{E}+00\end{array}$

Production/Consumption factors:

Produce: $<1$

Milk: 1.2

Meat: 2.9

FRACTION OF PGPULATION RECEIVING AN AVERAGE INDIVIDUAL TOTAL-BODY DOSE COMMITMENT FROM AIREORNE PATHWAYS

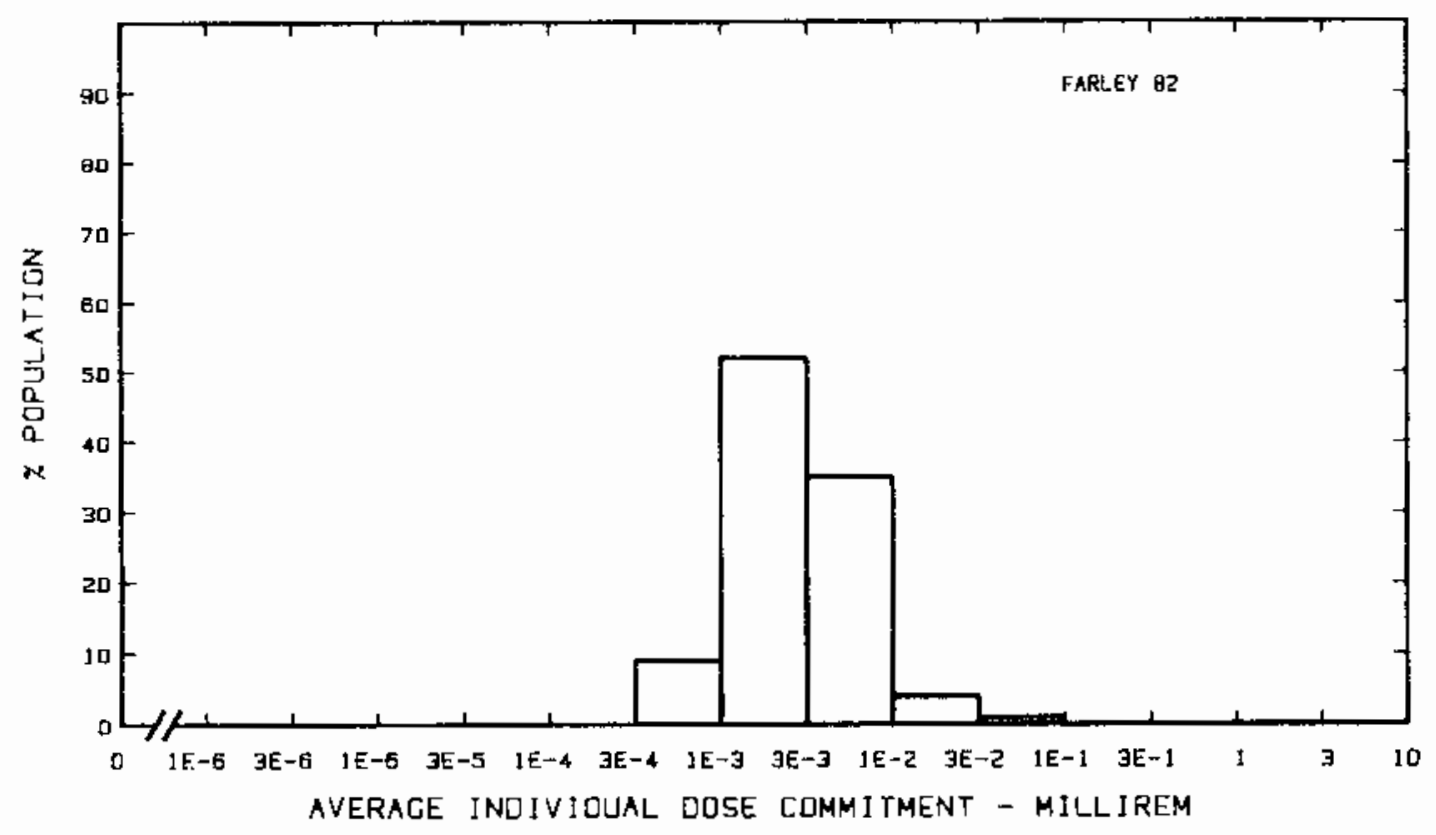


Site: J. A. FITZPATRICK

OSWEGO, NEW YORK

Location: $\quad N 43.5239^{\circ} \quad$ W $76.3983^{\circ}$

POPULATION DATA

Total Population Within 2-to-80-km Region: 8.4E5

Major Metropolitan Centers Within Region:

$\quad$ Center
Syracuse SMSA
Rome
Auburn
Watertown
Kingston

Population

Location

640,000

48,000

33,000

28,000

24,000

$56 \mathrm{~km} \quad \mathrm{SSE}$

$80 \mathrm{~km} \quad \mathrm{ESE}$

$66 \mathrm{~km} \quad \mathrm{SSW}$

$64 \mathrm{~km} \quad \mathrm{NE}$

$79 \mathrm{~km} \quad \mathrm{~N}$

SITE SPECIFIC DATA - AIRBORNE PATHWAYS

Average Annual State Production

Of Crops and Animal Products

In 80-km Radius Circle

Regional Productivity Factor:

Animat Grazing Factor:

Meteorology Period of Record: I JAN 74 - 3.1 DEC 75 Recovery: 97\%
Veg: $7.6 \mathrm{E} 7 \mathrm{kilogram}$

Milk: 7.0E8 liter

Meat: 3.3E7 kilogram

0.7

0.5

SITE SPECIFIC DATA - WATERBORNE PATHWAYS via LAKE ONTARIO

Average Dilution Flow

from Plant: $750 \mathrm{ft}^{3} / \mathrm{s}$

Drinking Water:

Exposed Population: $53 \rho_{B} 900$ (a)

Fish:

Edible Harvest: $7.3 \mathrm{E} 5 \mathrm{~kg} / \mathrm{yr}$
Dilution Factor: 0.005

(a) Population exposed to drinking water derived from Nine Mile Point FES (1974).

(b) Oilution factors derived from FES (1973). 
POPULATION DOSE-COMMITMENT ESTIMATES ANO

AVERAGE INDIVIDUAL DOSE-COMMITMENT HISTOGRAM FOR

\section{J. A. FITZPATRICK}

Dose Commitments (person-rem) from Liquid Pathways

\begin{tabular}{|c|c|c|c|c|c|}
\hline & Total Body & $\underline{G I-L L I}$ & Thyroid & Bone & Liver \\
\hline $\begin{array}{l}\text { Infant } \\
\text { Child } \\
\text { Teen } \\
\text { Adult }\end{array}$ & $\begin{array}{l}1.1 \mathrm{E}-04 \\
6.7 \mathrm{E}-03 \\
1.1 \mathrm{E}-02 \\
1.2 \mathrm{E}-01\end{array}$ & $\begin{array}{l}6.9 \mathrm{E}-05 \\
1.7 \mathrm{E}-03 \\
2.1 \mathrm{E}-03 \\
1.9 \mathrm{E}-02\end{array}$ & $\begin{array}{l}7.7 \mathrm{E}-05 \\
5.6 \mathrm{E}-04 \\
1.7 \mathrm{E}-04 \\
1.2 \mathrm{E}-03\end{array}$ & $\begin{array}{l}3.6 \mathrm{E}-04 \\
2.7 \mathrm{E}-02 \\
1.5 \mathrm{E}-02 \\
8.9 \mathrm{E}-02\end{array}$ & $\begin{array}{l}5.1 \mathrm{E}-04 \\
3.3 \mathrm{E}-02 \\
2.6 \mathrm{E}-02 \\
1.6 \mathrm{E}-01\end{array}$ \\
\hline TOTAL & 1. $3 \mathrm{E}-01$ & 2. $3 \mathrm{E}-02$ & 2. $0 \mathrm{E}-03$ & 1. $3 \mathrm{E}-01$ & $2.1 \mathrm{E}-01$ \\
\hline
\end{tabular}

Dose Comnitments (person-rem) from Airborne Pathways

Total Body GI-LLI Thyroid Bone Liver Lung

$\begin{array}{lllllll}\text { Infant } & 9.8 \mathrm{E}-02 & 9.7 \mathrm{E}-02 & 3.6 \mathrm{E}-01 & 1.0 \mathrm{E}-01 & 9.8 \mathrm{E}-02 & 1.0 \mathrm{E}-01 \\ \text { Child } & 1.1 \mathrm{E}+00 & 1.1 \mathrm{E}+00 & 2.8 \mathrm{E}+00 & 1.3 \mathrm{E}+00 & 1.1 \mathrm{E}+00 & 1.1 \mathrm{E}+00 \\ \text { Teen } & 7.9 \mathrm{E}-01 & 8.0 \mathrm{E}-01 & 1.4 \mathrm{E}+00 & 8.5 \mathrm{E}-01 & 7.9 \mathrm{E}-01 & 8.4 \mathrm{E}-01 \\ \text { Adult } & 4.8 \mathrm{E}+00 & 4.8 \mathrm{E}+00 & 6.9 \mathrm{E}+00 & 5.0 \mathrm{E}+00 & 4.8 \mathrm{E}+00 & 4.9 \mathrm{E}+00 \\ \text { TOTAL } & 6.8 \mathrm{E}+00 & 6.8 \mathrm{E}+00 & 1.2 \mathrm{E}+01 & 7.3 \mathrm{E}+00 & 6.8 \mathrm{E}+00 & 7.0 \mathrm{E}+00\end{array}$

Production/Consumption factors:

Produce: <1 Milk: $4.4 \quad$ Meat: <1

FRACTIDN OF POPULATION RECEIVING AN AVERAGE INDIVIDUAL TOTAL-BODY DDSE COMMITMENT FROM AIRBORNE PATHWAYS

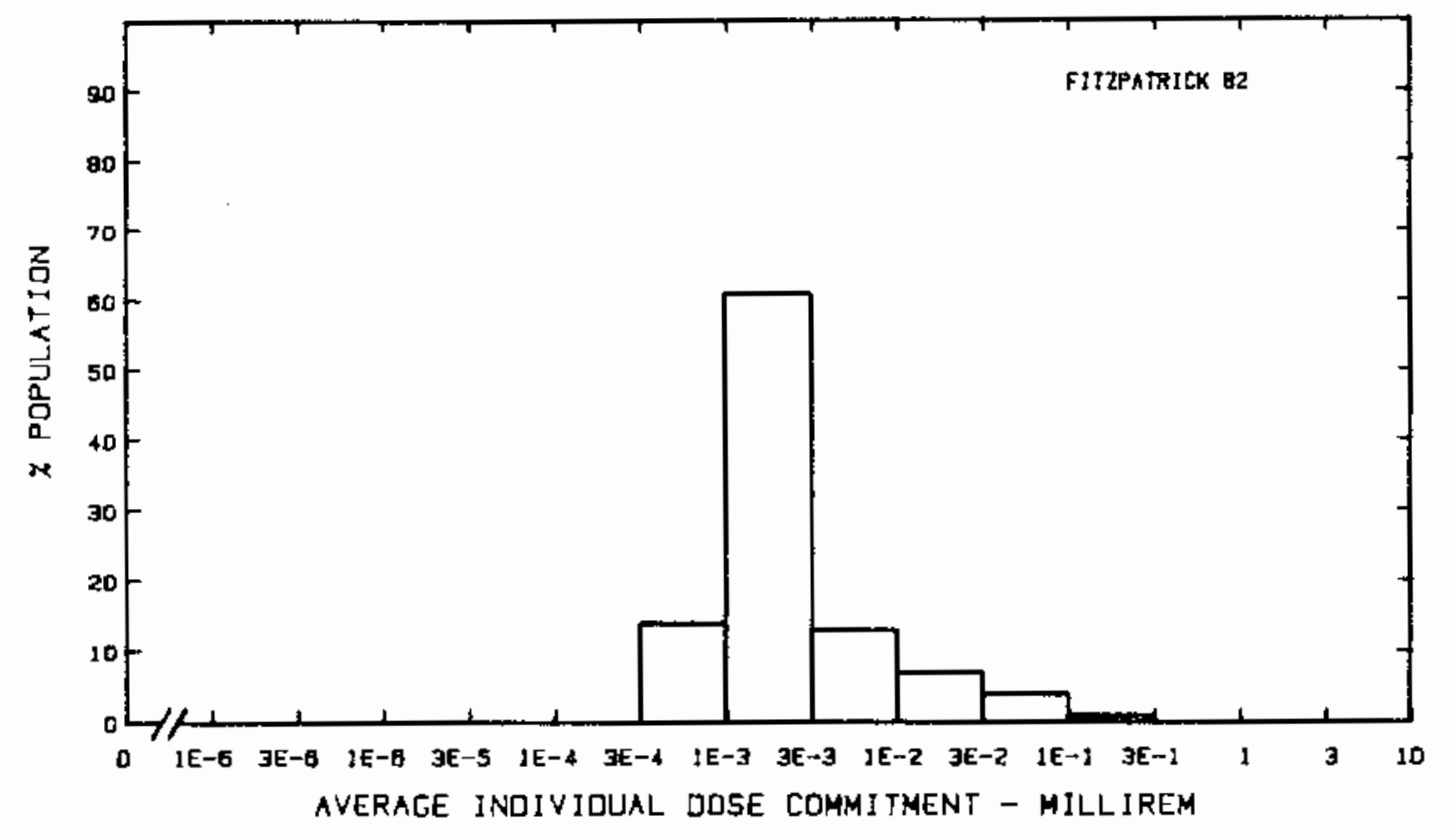


Site: FORT CALHOUN

WASHINGTON COUNTY, NEBRASKA

Location: N $41.5208^{\circ} \quad$ W $96.0 \% 67^{\circ}$

POPULATION DATA

Total Population Within 2-to-80-km Region: 7.6E5

Major Metropolitan Centers Within Region:

\begin{tabular}{lcccc}
\multicolumn{1}{c}{ Center } & Population & \multicolumn{2}{c}{ Location } \\
\cline { 2 - 3 } & & \multicolumn{2}{c}{} \\
Omaha & 570,000 & $32 \mathrm{~km}$ & SSE \\
Council Bluffs & 56,000 & $34 \mathrm{~km}$ & SE \\
Freemont & 24,000 & $36 \mathrm{~km}$ & WSW \\
Bellevue & 22,000 & $44 \mathrm{~km}$ & SSE
\end{tabular}

SITE SPECIFIC DATA - AIRBORNE PATHWAYS

Average Annual State Production

of Crops and Animal Products

In $80-k m$ Radius Circle

Regional Productivity Factor:

Animal Grazing Factor:

Meteorology Period of Record: 1 JAN $74-\$ 1$ DEC 74 Recovery: 98\%
Veg: $9.7 \mathrm{E} 7 \mathrm{kilogram}$

Milk: 7.2 E7 liter

Meat: $2.0 \mathrm{E} 8$ kilogram

1

0.5

SITE SPECIFIC OATA - WATERBORNE PATHWAYS via MISSDURI RIVER

Average River Flow

at Site: $27,000 \mathrm{ft}^{3} / \mathrm{s}$

Drinking Water:

Exposed Population: $570,000^{(a)}$

Dilution Factor: 1

Fish:

Edible Harvest: $1.0 \mathrm{E} 4 \mathrm{~kg} / \mathrm{yr}$

Dilution Factor: 1

(a) Drinking-water pollution assumed to be Dmaha SMSA (FES, 1972). 
POPULATION DOSE-COMMITMENT ESTIMATES ANO

AVERAGE INDIVIOUAL DOSE-COMMITMENT HISTOGRAM FOR

FORT CALHOUN

Dose Commitments (person-rem) from Liquid Pathways

\begin{tabular}{|c|c|c|c|c|c|}
\hline & Total Body & GI-LLI & Thyroid & Bone & Liver \\
\hline $\begin{array}{l}\text { Infant } \\
\text { Child } \\
\text { Teen } \\
\text { Adult }\end{array}$ & $\begin{array}{l}5.8 \mathrm{E}-03 \\
6.7 \mathrm{E}-02 \\
2.8 \mathrm{E}-02 \\
2.5 \mathrm{E}-01\end{array}$ & $\begin{array}{l}5.6 \mathrm{E}-03 \\
6.4 \mathrm{E}-02 \\
2.5 \mathrm{E}-02 \\
2.2 \mathrm{E}-01\end{array}$ & $\begin{array}{l}1.1 \mathrm{E}-02 \\
1.0 \mathrm{E}-01 \\
3.5 \mathrm{E}-02 \\
2.9 \mathrm{E}-01\end{array}$ & $\begin{array}{l}2.2 \mathrm{E}-03 \\
2.5 \mathrm{E}-02 \\
6.6 \mathrm{E}-03 \\
4.1 \mathrm{E}-02\end{array}$ & $\begin{array}{l}8.5 \mathrm{E}-03 \\
8.9 \mathrm{E}-02 \\
3.4 \mathrm{E}-02 \\
2.7 \mathrm{E}-01\end{array}$ \\
\hline TOTAL & 3. $5 E-01$ & $3.1 E-01$ & 4. 3E-01 & $7.4 E+02$ & 4. $0 \mathrm{E}-01$ \\
\hline
\end{tabular}

Dose Commitments (person-rem) from Airborne Pathways

Total Body GI-LLI Thyroid Bone Liver Lung

$\begin{array}{lllllll}\text { Infant } & 6.7 \mathrm{E}-05 & 6.6 \mathrm{E}-05 & 6.1 \mathrm{E}-04 & 5.9 \mathrm{E}-05 & 6.9 \mathrm{E}-05 & 7.1 \mathrm{E}-05 \\ \text { Child } & 8.5 \mathrm{E}-04 & 8.4 \mathrm{E}-04 & 6.3 \mathrm{E}-03 & 6.6 \mathrm{E}-04 & 8.7 \mathrm{E}-04 & 9.1 \mathrm{E}-04 \\ \text { Teen } & 6.0 \mathrm{E}-04 & 5.9 \mathrm{E}-04 & 2.7 \mathrm{E}-03 & 4.7 \mathrm{E}-04 & 6.0 \mathrm{E}-04 & 6.7 \mathrm{E}-04 \\ \text { AduIt } & 3.5 \mathrm{E}-03 & 3.5 \mathrm{E}-03 & 1.1 \mathrm{E}-02 & 2.8 \mathrm{E}-03 & 3.5 \mathrm{E}-03 & 3.8 \mathrm{E}-03 \\ \text { TOTAL } & 5.0 \mathrm{E}-03 & 5.0 \mathrm{E}-03 & 2.1 \mathrm{E}-02 & 4.0 \mathrm{E}+03 & 5.1 \mathrm{E}-03 & 5.4 \mathrm{E}-03\end{array}$

Production/Consumption factors:

Produce: $<1 \quad$ Milk: $<1 \quad$ Meat: 3.3

FRACTION OF POPULATIDN RECEIVING AN AVERACE INDIVIDUAL TOTAL-EDOY DDSE CDMMITMENT FROM AJREORNE PATHHAYS

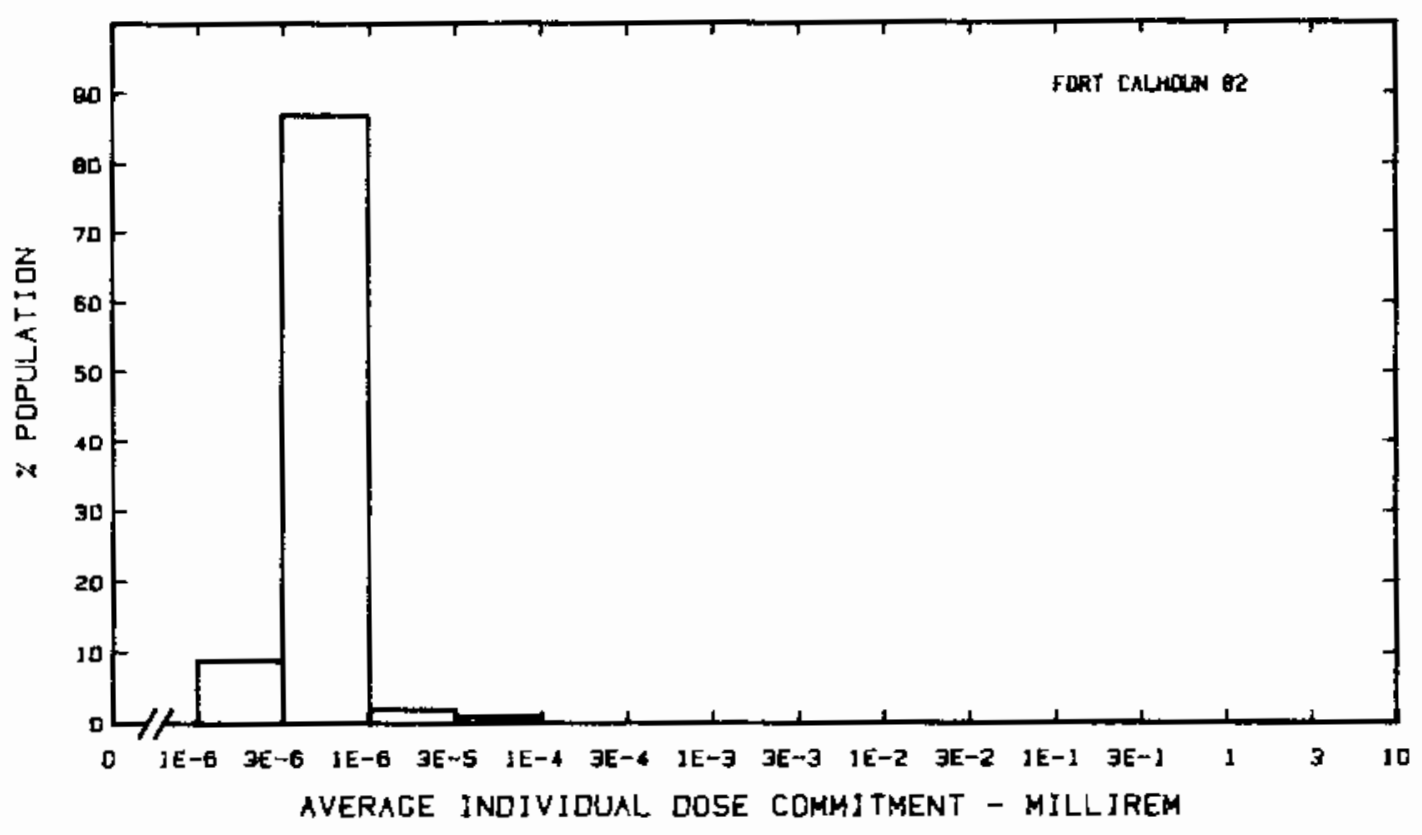


Site: R. E. GINNA

ONTARIO, NEW YORK

Location: N $43.2778^{\circ} \quad$ W $77.3089^{\circ}$

POPULATION DATA

Total Population Within 2-to-80-km Region: 1.2E6

Major Metropolitan Centers Within Region:

\begin{tabular}{lrrrr}
\multicolumn{1}{c}{ Center } & Population & & \multicolumn{2}{c}{ Location } \\
\cline { 5 - 7 } Rochester SMSA & 970,000 & & $27 \mathrm{~km}$ & WSW \\
Auburn & 32,000 & & $71 \mathrm{~km}$ & ESE \\
Oswego & 20,000 & $67 \mathrm{~km}$ & ENE \\
Batavia & 17,000 & $78 \mathrm{~km}$ & WSW \\
Geneva & 15,000 & $52 \mathrm{~km}$ & SSE
\end{tabular}

SITE SPECIFIC OATA - AIRBORNE PATHWAYS

Average Annual State Production

of Crops and Animal Products

In 80-km Radius Circle

Regional Productivity Factor:

Animal Grazing Factor:

Meteorology Period of Record: 1 JAN 66 - 31 OEC 67 Recovery: $89 \%$

SITE SPECIFIC OATA - WATERBORNE PATHWAYS via LAKE ONTARIO

Average 0ilution Flow

from Plant: $750 \mathrm{ft}^{3} / \mathrm{s}$

Orinking Water:

Exposed Population: 560,000

Fish:

Edible Harvest: $7.3 \mathrm{E} 5 \mathrm{~kg} / \mathrm{yr}$

Dilution Factor: 0.01

(a) Dilution factors from FES (1973). 
POPULATION DOSE-COMMITMENT ESTIMATES AND

AVERAGE INOIVIOUAL OOSE-COMMITMENT HISTOGRAM FOR

R. E. GINNA

Dose Commitments (person-rem) from Liquid Pathways

Total Body GI-LLI Thyroid Bone Liver

Infant $2.1 \mathrm{E}-03 \quad 2.4 \mathrm{E}-03 \quad 2.0 \mathrm{E}-03 \quad 8.7 \mathrm{E}-04 \quad 2.4 \mathrm{E}-03$

Child 2.6E-02 3.3E-02 2.2E-02 2.1E-02

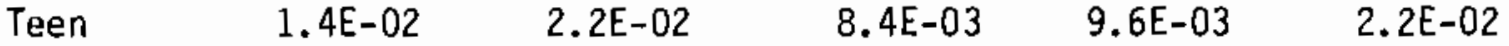

Adult $1.3 \mathrm{E}-01 \quad 1.9 \mathrm{E}-01 \quad 7.1 \mathrm{E}-02 \quad 5.6 \mathrm{E}-02 \quad 1.6 \mathrm{E}-01$

$\begin{array}{llllll}\text { TOTAL } & 1.8 \mathrm{E}-01 & 2.5 \mathrm{E}-01 & 1.0 \mathrm{E}-01 & 8.8 \mathrm{E}-02 & 2.2 \mathrm{E}-01\end{array}$

Dose Commitments (person-rem) from Airborne Pathways

Total Body GI-LLI Thyrojd Bone Liver Lung

$\begin{array}{lllllll}\text { Infant } & 7.5 \mathrm{E}-04 & 7.4 \mathrm{E}-04 & 1.2 \mathrm{E}-03 & 3.8 \mathrm{E}-04 & 7.5 \mathrm{E}-04 & 7.8 \mathrm{E}-04 \\ \text { Child } & 9.2 \mathrm{E}-03 & 9.2 \mathrm{E}-03 & 1.2 \mathrm{E}-02 & 4.2 \mathrm{E}-03 & 9.2 \mathrm{E}-03 & 9.7 \mathrm{E}-03 \\ \text { Teen } & 6.1 \mathrm{E}-03 & 6.1 \mathrm{E}-03 & 7.3 \mathrm{E}-03 & 3.1 \mathrm{E}-03 & 6.1 \mathrm{E}-03 & 6.8 \mathrm{E}-03 \\ \text { Adult } & 3.4 \mathrm{E}-02 & 3.4 \mathrm{E}-02 & 3.8 \mathrm{E}-02 & 1.8 \mathrm{E}-02 & 3.4 \mathrm{E}-02 & 3.7 \mathrm{E}-02 \\ \text { TOTAL } & 5.1 \mathrm{E}-02 & 5.1 \mathrm{E}-02 & 5.9 \mathrm{E}-02 & 2.6 \mathrm{E}-02 & 5.1 \mathrm{E}-02 & 5.4 \mathrm{E}-02\end{array}$

Production/Consumption factors:

Produce: <1 Milk: $2.7 \quad$ Meat: <1

FRACTION IF POPULATION RECEIVING AN AVERAGE INDJVIDUAL TOTAL-BOOY COSE COMMITMENT FROM AIRGORNE PATHWAYS

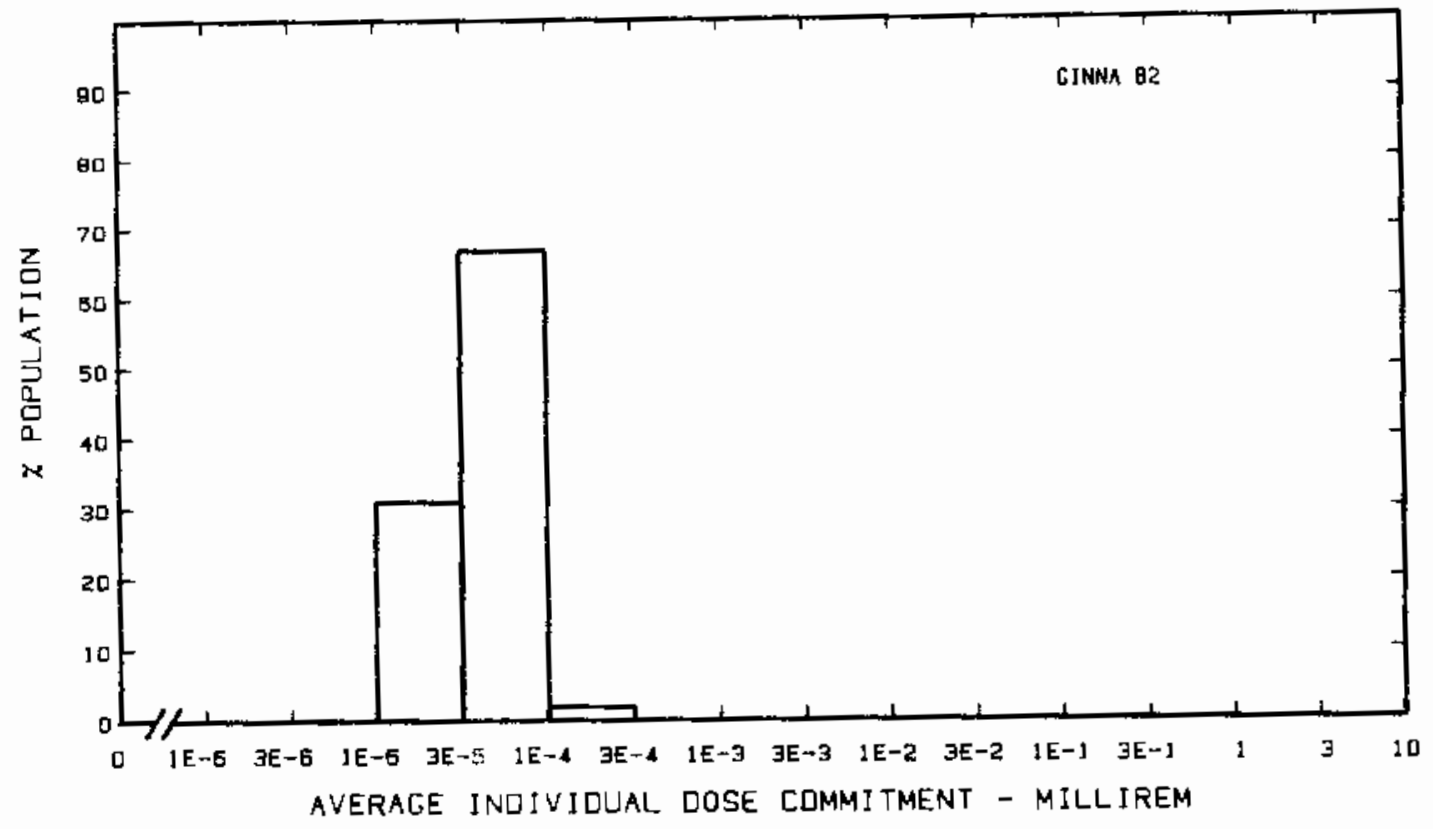


Site: HADDAM NECK

HADDAM NECK, CONNECTICUT

Location: N $41.4819^{\circ}$ W $72.4992^{\circ}$

POPULATION DATA

Total Population Within 2-to-80-km Region: $3.4 \mathrm{E} 6$

Major Metropolitan Centers Within Region:

Center

Hartford SMSA

Springfield-Chicopee-Holyoke SMSA

New Haven-West Haven SMSA

Bridgeport SMSA

New London-Norwich
Population

730,000

530,000

420,000

400,000

250,000
Location

$35 \mathrm{~km} \quad \mathrm{NNW}$

$70 \mathrm{~km} \quad \mathrm{~N}$

$40 \mathrm{~km}$ WSW

$66 \mathrm{~km}$ WSW

$35 \mathrm{~km}$ ESE

\section{SITE SPECIFIC DATA - AIRBORNE PATHWAYS}

Average Annual State Production

of Crops and Animal Products

In $80-\mathrm{km}$ Radius Circle

Regional Productivity Factor:

Animal Grazing Factor:

Meteorology Period of Record: 1 JAN 75 - 31 DEC 75 Recovery: 95\%
Veg: $3.2 E 7$ kilogram

Mijk: 4.4E8 liter

Meat: $2.0 E 7$ kilogram

0.7

0.6

SITE SPECIFIC DATA - WATERBORNE PATHWAYS via DISCHARGE CANAL. TO CONN. RIVER

Average Discharge Canal

Flow at Site: $920 \mathrm{ft}^{3} / \mathrm{s}$

Drinking Water:

Exposed Population: None

Fish:

Edible Harvest: $9.1 \mathrm{E3^{( \textrm {a } ) }} \mathrm{kg} / \mathrm{yr}$

Dilution Factor: 1

(a) Caught in discharge canal (FES, 1973). 
POPULATION DOSE-COMMITMENT ESTIMATES AND

AVERAGE INDIVIDUAL DOSE-COMMITMENT HISTOGRAM FOR

\section{HADDAM NECK}

Dose Commitments (person-rem) from Liquid Pathways

\begin{tabular}{|c|c|c|c|c|c|}
\hline & Tota1 Body & $\underline{G I-L L I}$ & Thyroid & Bone & Liver \\
\hline $\begin{array}{l}\text { Infant } \\
\text { Child } \\
\text { Teen } \\
\text { Adult }\end{array}$ & $\begin{array}{l}0.0 \mathrm{E}+00 \\
2.1 \mathrm{E}-03 \\
2.9 \mathrm{E}-03 \\
2.8 \mathrm{E}-02\end{array}$ & $\begin{array}{l}0.0 E+00 \\
6.1 E-04 \\
6.7 E-04 \\
5.4 E-03\end{array}$ & $\begin{array}{l}0.0 \mathrm{E}+00 \\
4.9 \mathrm{E}-04 \\
4.4 \mathrm{E}-04 \\
3.5 \mathrm{E}-03\end{array}$ & $\begin{array}{l}0.0 \mathrm{E}+00 \\
8.6 \mathrm{E}-03 \\
5.8 \mathrm{E}-03 \\
3.6 \mathrm{E}-02\end{array}$ & $\begin{array}{l}0.0 \mathrm{E}+00 \\
6.7 \mathrm{E}-03 \\
5.6 \mathrm{E}-03 \\
3.4 \mathrm{E}-02\end{array}$ \\
\hline TOTAL & $3.3 \mathrm{E}-02$ & $6.7 \mathrm{E}-03$ & 4. $5 E-03$ & $5.1 E-02$ & $4.6 \mathrm{E}-02$ \\
\hline
\end{tabular}

Dose Commitments (person-rem) from Airborne Pathways

Total Body GI-LLI Thyroid Bone Liver Lung

$\begin{array}{lllllll}\text { Infant } & 2.4 \mathrm{E}-03 & 2.4 \mathrm{E}-03 & 2.7 \mathrm{E}-03 & 1.2 \mathrm{E}-03 & 2.4 \mathrm{E}-03 & 2.6 \mathrm{E}-03 \\ \text { Child } & 2.9 \mathrm{E}-02 & 2.9 \mathrm{E}-02 & 3.1 \mathrm{E}-02 & 1.4 \mathrm{E}-02 & 2.9 \mathrm{E}-02 & 3.1 \mathrm{E}-02 \\ \text { Teen } & 2.1 \mathrm{E}-02 & 2.1 \mathrm{E}-02 & 2.1 \mathrm{E}-02 & 1.0 \mathrm{E}-02 & 2.1 \mathrm{E}-02 & 2.3 \mathrm{E}-02 \\ \text { Adult } & 1.2 \mathrm{E}-01 & 1.2 \mathrm{E}-01 & 1.2 \mathrm{E}-01 & 6.1 \mathrm{E}-02 & 1.2 \mathrm{E}-01 & 1.3 \mathrm{E}-01 \\ \text { TOTAL } & 1.7 \mathrm{E}-01 & 1.7 \mathrm{E}-01 & 1.8 \mathrm{E}-01 & 8.6 \mathrm{E}-02 & 1.7 \mathrm{E}-01 & 1.8 \mathrm{E}-01\end{array}$

Production/Consumption factors:

Produce: $<1 \quad$ Milk: $<1 \quad$ Meat: $<1$

FRACTION OF POPULATION RECEIVING AN AVERAGE INDIVIDUAL TOTAL-6ODY DOSE COMMITMENT FRDM AIRBORNE PATHWAYS

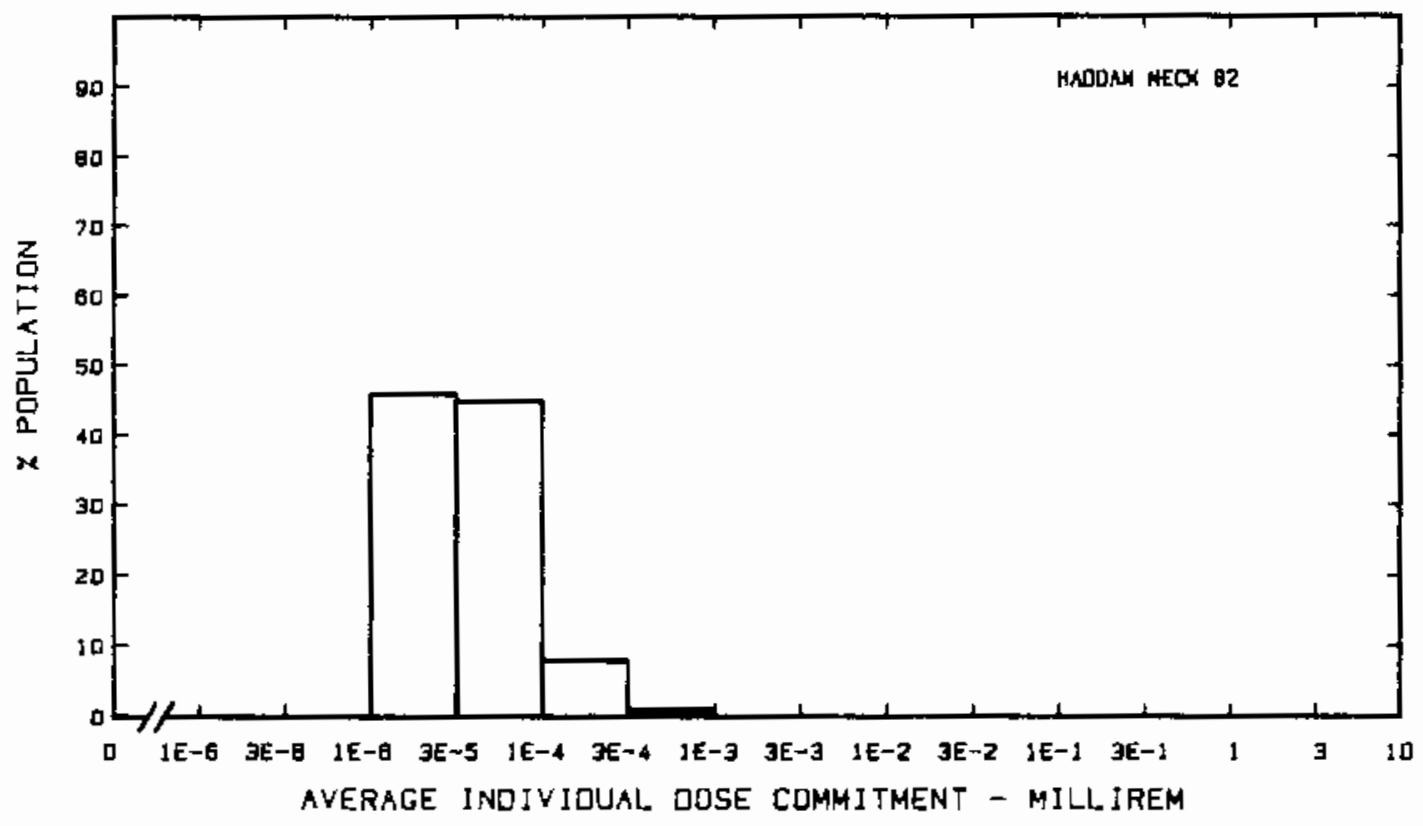


Site: E. I. HATCH

BAXI-EY, GEORGIA

Location: N $31.9342^{\circ}$ W $82.3444^{\circ}$

POPULATION DATA

Total Population Within 2-to-80-km Region: 3.0E5

Major Metropolitan Centers Within Region:

\begin{tabular}{lcccc} 
Center & Population & & \multicolumn{2}{c}{ Location } \\
\cline { 2 - 5 } & & & \\
Waycross & 19,000 & $80 \mathrm{~km}$ & $\mathrm{~S}$ \\
Statesboro & 15,000 & & $78 \mathrm{~km}$ & $\mathrm{NE}$ \\
Hinesville & 11,000 & $71 \mathrm{~km}$ & E \\
Douglas & 11,000 & $67 \mathrm{~km}$ & $\mathrm{SW}$ \\
Vidalia & 10,000 & $32 \mathrm{~km}$ & $\mathrm{~N}$
\end{tabular}

SITE SPECIFIC DATA - AIRBORNE PATHWAYS

Average Annual State Production

of Crops and Animal Products

In 80-km Radius Circle

Regional Productivity Factor:

Animal Grazing Factor:
Veg: $8.8 \mathrm{E} 6 \mathrm{kilogram}$

Milk: 7.0E7 liter

Meat: 8.1E7 kilogram

1

0.8

Meteorology Period of Record: 1 JUN 70 - 31 AUG 74 Recovery: 87\%

SITE SPECIFIC DATA - WATERBORNE PATHWAYS via ALTAMAHA RIVER

Average River Flow

at Site: $13,000 \mathrm{ft}^{3} / \mathrm{s}$

Drinking Water:

Exposed Population: None

Fish:

Edible Harvest: $6.3 \mathrm{ES}^{(\mathrm{a})} \mathrm{kg} / \mathrm{yr}$ Dilution Factor: 1

(a) Commercial catch plus 3 pounds of game fish per year taken from river by average person (FES, 1972). 
POPULATION DOSE-COMMITMENT ESTIMATES AND

AVERAGE INDIVIDUAL DOSE-COMMITMENT HISTOGRAM FOR

\author{
E. I. HATCH 1 AND 2
}

Dose Commitments (person-rem) from Liquid Pathways

Total Body GI-LLI Ihyroid Bone Liver

$\begin{array}{llllll}\text { Infant } & 0.0 \mathrm{E}+00 & 0.0 \mathrm{E}+00 & 0.0 \mathrm{E}+00 & 0.0 \mathrm{E}+00 & 0.0 \mathrm{E}+00 \\ \text { Child } & 1.4 \mathrm{E}-01 & 1.5 \mathrm{E}-02 & 1.6 \mathrm{E}-02 & 5.4 \mathrm{E}-01 & 6.6 \mathrm{E}-01 \\ \text { Teen } & 2.4 \mathrm{E}-01 & 3.2 \mathrm{E}-02 & 1.1 \mathrm{E}-02 & 3.3 \mathrm{E}-01 & 5.7 \mathrm{E}-01 \\ \text { Adult } & 2.4 \mathrm{E}+00 & 2.8 \mathrm{E}-01 & 7.4 \mathrm{E}-02 & 1.9 \mathrm{E}+00 & 3.4 \mathrm{E}+00 \\ \text { TOTAL } & 2.8 \mathrm{E}+00 & 3.3 \mathrm{E}-01 & 1.0 \mathrm{E}-01 & 2.8 \mathrm{E}+00 & 4.6 \mathrm{E}+00\end{array}$

Dose Commitments (person-rem) from Airborne Pathways

Total Body GI-LLI Thyroid Bone Liver Lung

$\begin{array}{lllllll}\text { Infant } & 6.6 \mathrm{E}-04 & 5.7 \mathrm{E}-04 & 7.3 \mathrm{E}-02 & 6.9 \mathrm{E}-04 & 9.0 \mathrm{E}-04 & 6.0 \mathrm{E}-04 \\ \text { Child } & 7.5 \mathrm{E}-03 & 6.8 \mathrm{E}-03 & 4.1 \mathrm{E}-01 & 6.6 \mathrm{E}-03 & 8.7 \mathrm{E}-03 & 7.1 \mathrm{E}-03 \\ \text { Teen } & 5.1 \mathrm{E}-03 & 4.8 \mathrm{E}-03 & 1.6 \mathrm{E}-01 & 4.0 \mathrm{E}-03 & 5.6 \mathrm{E}-03 & 5.2 \mathrm{E}-03 \\ \text { Adult } & 2.9 \mathrm{E}-02 & 2.8 \mathrm{E}-02 & 5.3 \mathrm{E}-01 & 2.2 \mathrm{E}-02 & 3.0 \mathrm{E}-02 & 2.9 \mathrm{E}-02 \\ \text { TOTAL } & 4.2 \mathrm{E}-02 & 4.0 \mathrm{E}-02 & 1.2 \mathrm{E}+00 & 3.3 \mathrm{E}-02 & 4.5 \mathrm{E}-02 & 4.2 \mathrm{E}-02\end{array}$

Production/Consumption factors:

Produce: <1 Milk: $1.8 \quad$ Meat: 3.3

FRACTION OF POPULATION RECEIVING AN AVERAGE INDIVIDUAL TOTAL-BODY DOSE CDMMITMENT FROM AJRBORNE PATHWAYS

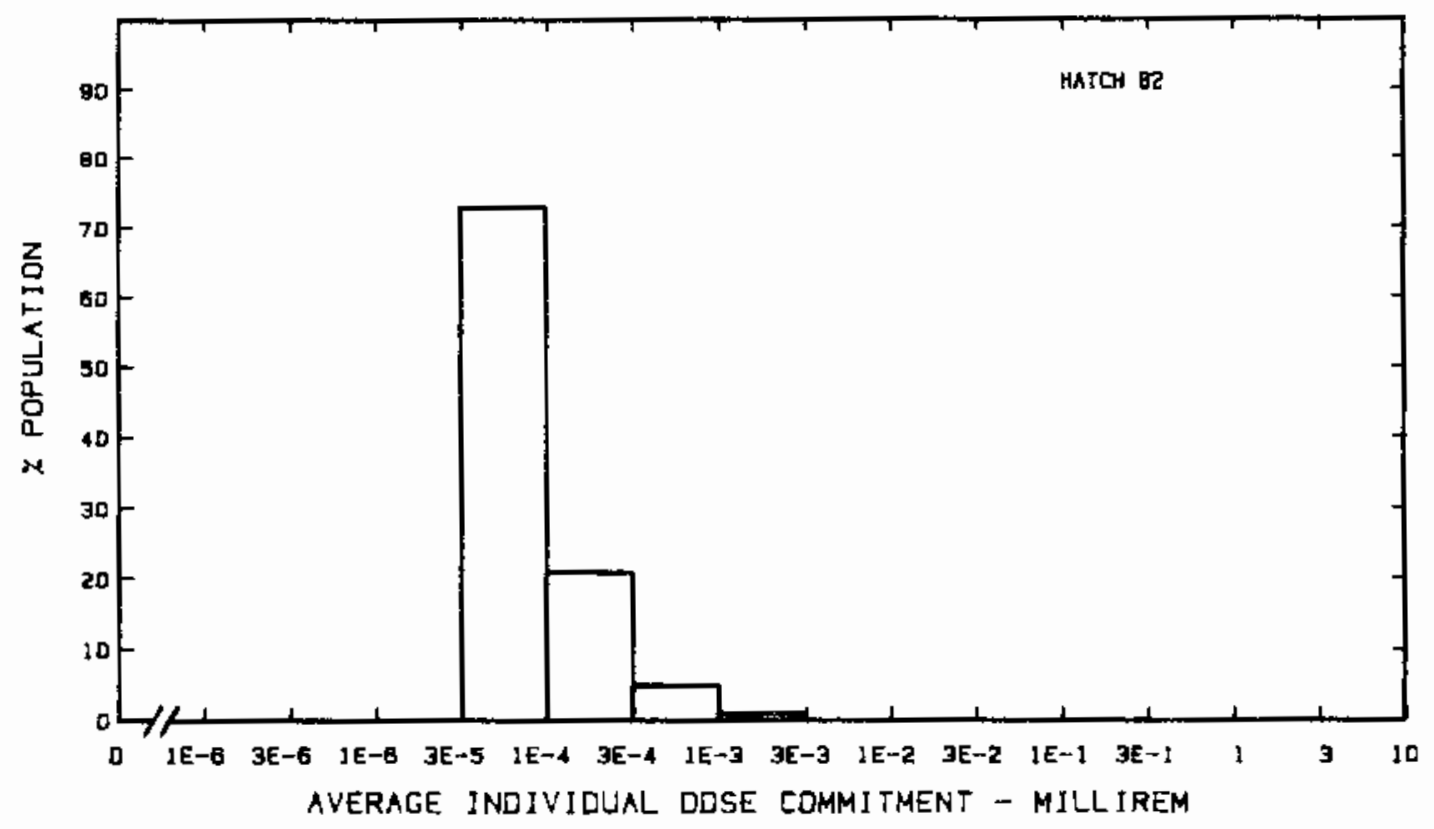

2.37 
Site: INDIAN POINT

BUCHANAN, NEW YORK
Location:
N $41.2714^{\circ}$
W $73.9525^{\circ}$

POPULATION DATA

Total Population Within 2-to-80-km Region: 1.5E7

Major Metropolitan Centers Within Region:

Center Population Location

$\begin{array}{lrrl}\text { New York SMSA } & 9,100,000 & 57 \mathrm{~km} & \mathrm{~S} \\ \text { Newark SMSA } & 2,000,000 & 62 \mathrm{~km} & \text { SSW } \\ \text { Nassau County SMSA } & 1,300,000 & 70 \mathrm{~km} & \text { SSE } \\ \text { Jersey City SMSA } & 560,000 & 61 \mathrm{~km} & \mathrm{~S} \\ \text { Paterson-Clifton-Passaic SMSA } & 450,000 & 44 \mathrm{~km} & \text { SSW }\end{array}$

SITE SPECIFIC DATA - AIRBORNE PATHWAYS

Average Annual State Production

of Crops and Animal Products

In 80-km Radius Circle

Veg: $7.6 \mathrm{E} 7 \mathrm{ki}$ logram

MiIk: 7.0E8 liter

Meat: $3.3 \mathrm{E} 7$ kilogram

Regional Productivity Factor: $\quad 0.8$

Animal Grazing Factor: $\quad 0.5$

Meteorology Period of Record: 1 JAN 75 - 31 DEC 75 Recovery: 96\%

SITE SPECIFIC DATA - WATERBORNE PATHWAYS via HUDSON RIVER

Average River Flow

at Site: $2 \mathrm{D}, 000 \mathrm{ft}^{3} / \mathrm{s}$

Drinking Water: Exposed Population: None

Fish: $\quad$ Edible Harvest: (a)

Dilution Factor: $0.001^{(b)}$

(a) No fish catch data given in FES, so generic consumption rates used (Table A-1).

(b) One percent of population obtain $10 \%$ of their fish from river (FES, 1972). 
POPULATION DOSE-COMMITMENT ESTIMATES ANO

AVERAGE INDIVIDUAL OOSE-COMMITMENT HISTOGRAM FOR

INOIAN POINT 1,2 ANO 3

Dose Commitments (person-rem) from Liquid Pathways

\begin{tabular}{|c|c|c|c|c|c|}
\hline & Tota] Body & GI-LLI & Thyroid & Bone & Liver \\
\hline $\begin{array}{l}\text { Infant } \\
\text { Child } \\
\text { Teen } \\
\text { Adult }\end{array}$ & $\begin{array}{l}0.0 \mathrm{E}+00 \\
3.0 \mathrm{E}-02 \\
5.7 \mathrm{E}-02 \\
6.2 \mathrm{E}-01\end{array}$ & $\begin{array}{l}0.0 \mathrm{E}+00 \\
3.1 \mathrm{E}-03 \\
6.3 \mathrm{E}-03 \\
5.4 \mathrm{E}-02\end{array}$ & $\begin{array}{l}0.0 \mathrm{E}+00 \\
8.3 \mathrm{E}-04 \\
6.0 \mathrm{E}-04 \\
4.0 \mathrm{E}-03\end{array}$ & $\begin{array}{l}0.0 \mathrm{E}+00 \\
1.6 \mathrm{E}-01 \\
9.3 \mathrm{E}-02 \\
5.4 \mathrm{E}-01\end{array}$ & $\begin{array}{l}0.0 \mathrm{E}+00 \\
1.7 \mathrm{E}-01 \\
1.5 \mathrm{E}-01 \\
8.6 \mathrm{E}-01\end{array}$ \\
\hline TOTAL & $7.0 \mathrm{E}-01$ & $6.3 \mathrm{E}-02$ & $5.4 \mathrm{E}-03$ & $7.9 \mathrm{E}-01$ & $1.2 E-0$ \\
\hline
\end{tabular}

Dose Commitments (person-rem) from Airborne Pathways

Total Body GI-LLI Thyroid Bone Liver Lung

$\begin{array}{llllllll}\text { Infant } & 6.9 \mathrm{E}-02 & 6.9 \mathrm{E}-02 & 1.7 \mathrm{E}-01 & 6.9 \mathrm{E}-02 & 7.0 \mathrm{E}-02 & 7.5 \mathrm{E}-02 \\ \text { Child } & 7.7 \mathrm{E}-0 \mathrm{I} & 7.7 \mathrm{E}-01 & 1.5 \mathrm{E}+00 & 7.7 \mathrm{E}-01 & 7.7 \mathrm{E}-01 & 8.6 \mathrm{E}-01 \\ \text { Teen } & 5.6 \mathrm{E}-01 & 5.6 \mathrm{E}-01 & 9.4 \mathrm{E}-01 & 5.6 \mathrm{E}-01 & 5.6 \mathrm{E}-01 & 6.8 \mathrm{E}-01 \\ \text { Adult } & 3.4 \mathrm{E}+00 & 3.4 \mathrm{E}+00 & 5.0 \mathrm{E}+00 & 3.4 \mathrm{E}+00 & 3.4 \mathrm{E}+00 & 3.8 \mathrm{E}+00 \\ \text { TOTAL } & 4.8 \mathrm{E}+00 & 4.8 \mathrm{E}+00 & 7.6 \mathrm{E}+00 & 4.8 \mathrm{E}+00 & 4.8 \mathrm{E}+00 & 5.4 \mathrm{E}+00\end{array}$

Production/Consumption factors:

Produce: $<1 \quad$ Milk: $<i \quad$ Meat: $<1$

FRACTIDN OF PDP!ILATION RECEIVING AN AVERACEE INDIVIDUAL

TOTAL-BOCY DOSE COMMITMENT FRDM AIRGQRNE PATHWAYS

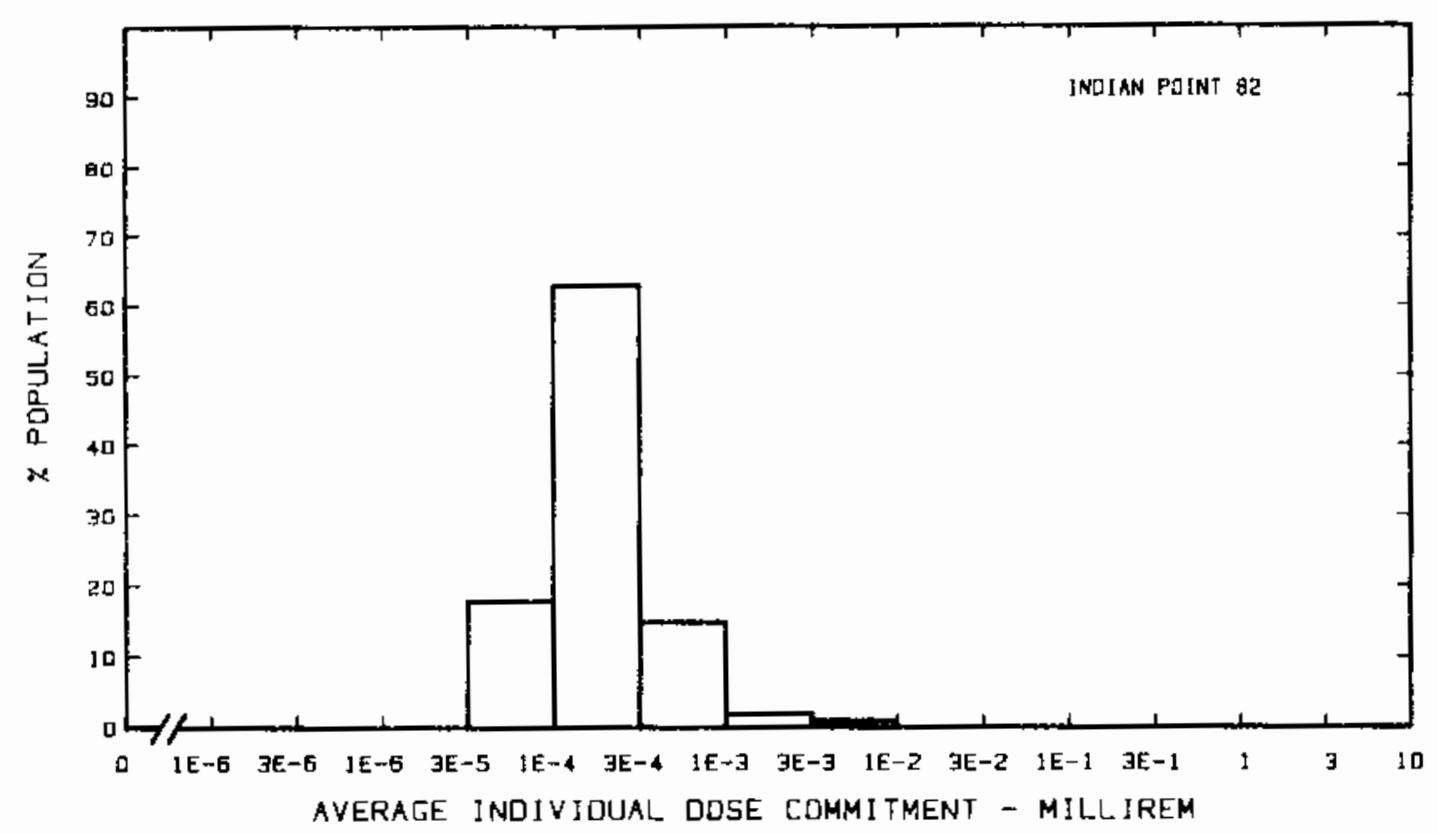


Site: KEWAUNEE

CARLTON, WISCONSIN

Location: N $44.3431^{\circ}$ W $87.536^{\circ}$

POPULATION DATA

Total Population Within 2-to-80-km Region: $6.2 E 5$

Major Metropolitan Centers Witnin Region:

Center

Greenbay SMSA

Appleton SMSA

Sheboygan

Manitowoc

Neenah
Population

180,000

290,000

48,000

33,000

22,000
Location

$44 \mathrm{~km} \quad \mathrm{NW}$

$72 \mathrm{~km} \quad \mathrm{~W}$

$65 \mathrm{~km} \quad \mathrm{SSW}$

$29 \mathrm{~km} \quad \mathrm{SSW}$

$72 \mathrm{~km}$ W

SITE SPECIFIC DATA - AIRBORNE PATHWAYS

Average Annual State Production

of Crops and Animal Products

In 80-km Radius Circle

Regional Productivity Factor:

Animal Grazing Factor:
Veg: $7.2 \mathrm{E} 7 \mathrm{kilogram}$

Milk: 1.2Eg liter

Meat: $1.0 \mathrm{E} 8$ kilogram

0.5

0.5

Meteorology Period of Record: 2 JAN 69 - 31 DEC 69 Recovery: 76\%

SITE SPECIFIC DATA - WATERBORNE PATHWAYS via LAKE MICHIGAN

Average Dilution Flow from

Plant: $63 \mathrm{ft}^{3} / \mathrm{s}$

Drinking Water:

Exposed Population: $260(890$
Dilution Factor: $8.2 \mathrm{E}-3$

Fish:

Edible Harvest: $1.1(\mathrm{~b})$
Dilution Factor: 0.01
$(\mathrm{c}) / \mathrm{yr}$

(a) Drinking-water dilution factor estimated by averaging dilution factors derived from FES (1972) suitably weighted for populations.

(b) Average individual consumption rate as given in the FES (1972) used in lieu of catch data.

(c) Dilution factor reduced $1 / 10$ from that used in FES in consideration of lake mixing. 


\section{POPULATION DOSE-COMMITMENT ESTIMATES AND \\ AVERAGE INDIVIDUAL DOSE-COMMITMENT HISTOGRAM FOR \\ KEWAUNEE}

Dose Commitments (person-rem) from Liquid Pathways

Total Body GI-LLI Thyroid Bone Liver

$\begin{array}{llllll}\text { Infant } & 1.1 \mathrm{E}-02 & 1.2 \mathrm{E}-02 & 9.2 \mathrm{E}-03 & 2.9 \mathrm{E}-03 & 1.3 \mathrm{E}-02 \\ \text { Child } & 1.7 \mathrm{E}-01 & 1.7 \mathrm{E}-01 & 1.0 \mathrm{E}-01 & 2.8 \mathrm{E}-01 & 4.1 \mathrm{E}-01 \\ \text { Teen } & 1.4 \mathrm{E}-01 & 1.2 \mathrm{E}-01 & 4.0 \mathrm{E}-02 & 1.6 \mathrm{E}-01 & 2.8 \mathrm{E}-01 \\ \text { Adult } & 1.4 \mathrm{E}+01 & 1.0 \mathrm{E}+00 & 3.4 \mathrm{E}-01 & 9.2 \mathrm{E}-01 & 1.8 \mathrm{E}+00 \\ \text { TOTAL } & 1.7 \mathrm{E}+00 & 1.3 \mathrm{E}+00 & 4.9 \mathrm{E}-01 & 1.4 \mathrm{E}+00 & 2.4 \mathrm{E}+00\end{array}$

Dose Commitments (person-rem) from Airborne Pathways

Total Body GI-LLI Thyroid Bone Liver Lung

$\begin{array}{lllllll}\text { Infant } & 3.8 \mathrm{E}-05 & 3.8 \mathrm{E}-05 & 4.0 \mathrm{E}-05 & 8.8 \mathrm{E}-06 & 3.8 \mathrm{E}-05 & 3.9 \mathrm{E}-05 \\ \text { Child } & 5.7 \mathrm{E}-04 & 5.6 \mathrm{E}-04 & 5.8 \mathrm{E}-04 & 1.2 \mathrm{E}-04 & 5.6 \mathrm{E}-04 & 5.9 \mathrm{E}-04 \\ \text { Teen } & 3.5 \mathrm{E}-04 & 3.5 \mathrm{E}-04 & 3.6 \mathrm{E}-04 & 8.3 \mathrm{E}-05 & 3.5 \mathrm{E}-04 & 3.8 \mathrm{E}-04 \\ \text { Adult } & 2.0 \mathrm{E}-03 & 1.9 \mathrm{E}-03 & 2.0 \mathrm{E}-03 & 4.8 \mathrm{E}-04 & 1.9 \mathrm{E}-03 & 2.0 \mathrm{E}-03 \\ \text { TOTAL } & 2.9 \mathrm{E}-03 & 2.9 \mathrm{E}-03 & 2.9 \mathrm{E}-03 & 6.9 \mathrm{E}-04 & 2.9 \mathrm{E}-03 & 3.0 \mathrm{E}-03\end{array}$

Production/Consumption factors:

Produce: $<1 \quad$ Milk: $7.2 \quad$ Meat: 1.0

FRACTION DF POPULATION RECEIVING AN AVERAGE INDIVIOUAL TOTAL-BODY DOSE COMMITMENT FROM AIRBORNE PATHWAYS

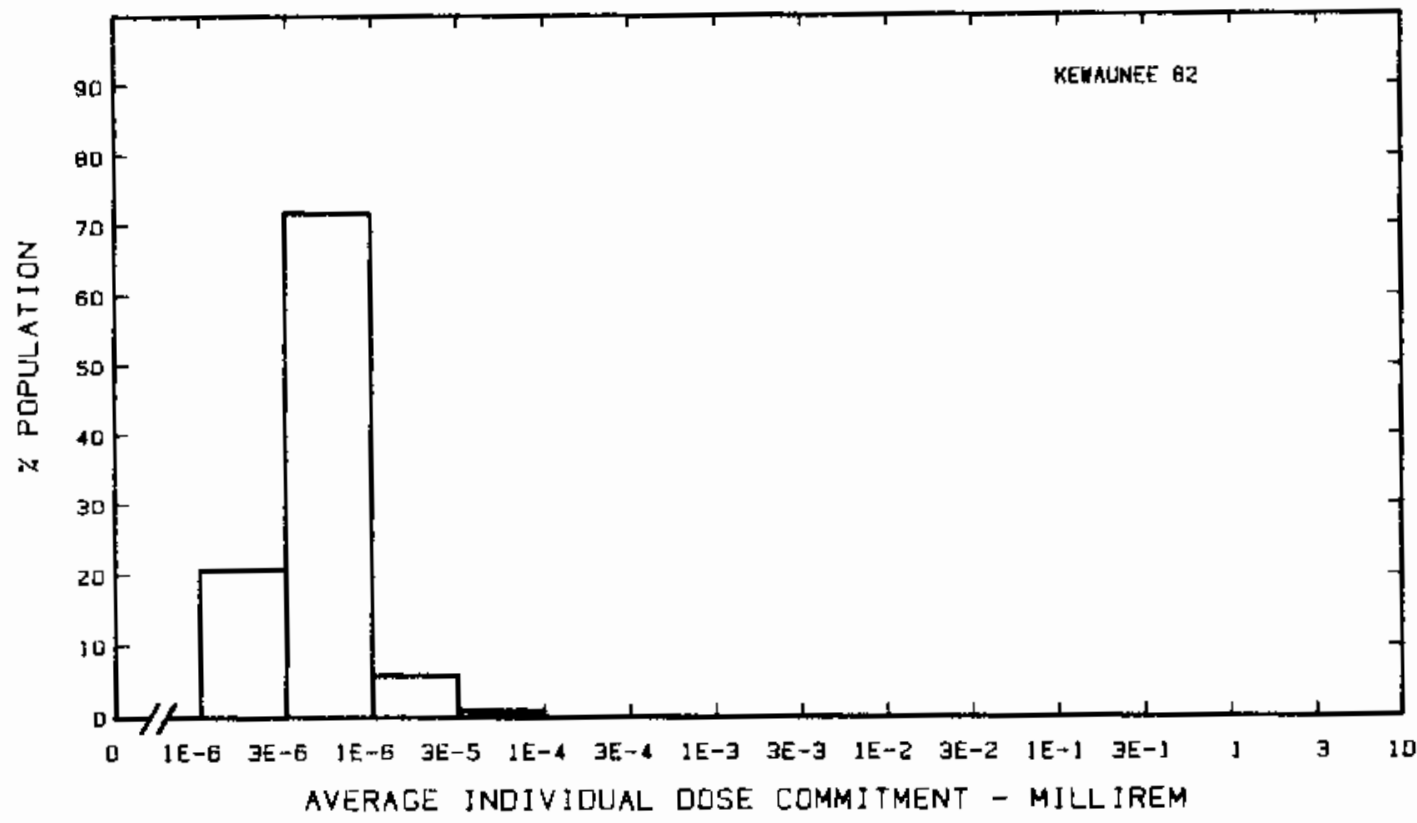


Site: LACROSSE

Location:

N $43.5583^{\circ}$
GENOA, WISCONSIN

W $91.2306^{\circ}$

POPULATION DATA

Total Population Within 2-to-80-km Region: 3.4E5

Major Metropolitan Centers Within Region:

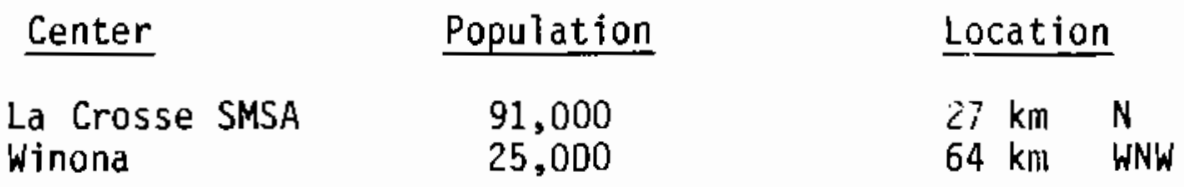

SITE SPECIFIC DATA - AIRBORNE PATHWAYS

Average Annual State Production

Of Crops and Animal Products

In 80-km Radius Circle

Regional Productivity Factor:

Animal Grazing Factor:

Meteorology Period of Record: 1 JAN 75 - 31 DEC 75 Recovery: 97\%
Veg: $7.2 \mathrm{E} 7 \mathrm{kilogram}$

Mijk: 1.2E9 liter

Meat: 1.DE8 kilogram

1

0.5

SITE SPECIFIC DATA - WATERBORNE PATHWAYS via MISSISSIPPI RIVER

Average River

Flow at Site: $28,000 \mathrm{ft}^{3} / \mathrm{s}$

Drinking Water:

Exposed Population: None

Fish:

Edible Harvest: (a) $k q / y^{r}$

(a) No fish catch data given in FES, so $1 / 2$ population assumed to consume river fish at generic consumption rates (Table A-1). 
POPULATION OOSE-COMMITMENT ESTIMATES ANO

AVERAGE INDIVIDUAL OOSE-COMMITMENT HISTOGRAM FOR

LACROSSE

Dose Commitments (person-rem) from Liquid Pathways

Total Body GI-LLI $\underline{\text { Thyroid }}$ Bone Liver

$\begin{array}{llllll}\text { Infant } & 0.0 \mathrm{E}+00 & 0.0 \mathrm{E}+00 & 0.0 \mathrm{E}+00 & 0.0 \mathrm{E}+00 & 0.0 \mathrm{E}+00 \\ \text { Child } & 2.9 \mathrm{E}-01 & 1.3 \mathrm{E}-01 & 1.3 \mathrm{E}-03 & 1.7 \mathrm{E}+00 & 1.8 \mathrm{E}+00 \\ \text { Teen } & 5.4 \mathrm{E}-01 & 2.7 \mathrm{E}-01 & 9.2 \mathrm{E}-04 & 1.0 \mathrm{E}+00 & 1.5 \mathrm{E}+00 \\ \text { Adult } & 5.9 \mathrm{E}+00 & 2.3 \mathrm{E}+00 & 6.2 \mathrm{E}-03 & 6.0 \mathrm{E}+00 & 8.7 \mathrm{E}+00 \\ \text { TOTAL } & 6.7 \mathrm{E}+00 & 2.7 \mathrm{E}+00 & 8.2 \mathrm{E}-03 & 8.7 \mathrm{E}+00 & 1.2 \mathrm{E}+01\end{array}$

Dose Commitments (person-rem) from Airborne Pathways Total Body GI-LLI Thyroid Bone Liver Lung

$\begin{array}{lllllll}\text { Infant } & 3.5 \mathrm{E}-03 & 3.5 \mathrm{E}-03 & 4.0 \mathrm{E}-03 & 3.5 \mathrm{E}-03 & 3.5 \mathrm{E}-03 & 3.6 \mathrm{E}-03 \\ \text { Child } & 4.0 \mathrm{E}-02 & 3.9 \mathrm{E}-02 & 4.4 \mathrm{E}-02 & 4.0 \mathrm{E}-02 & 3.9 \mathrm{E}-02 & 4.1 \mathrm{E}-02 \\ \text { Teen } & 2.9 \mathrm{E}-02 & 2.9 \mathrm{E}-02 & 3.1 \mathrm{E}-02 & 2.9 \mathrm{E}-02 & 2.9 \mathrm{E}-02 & 3.0 \mathrm{E}-02 \\ \text { Adult } & 1.7 \mathrm{E}-01 & 1.7 \mathrm{E}-01 & 1.8 \mathrm{E}-01 & 1.7 \mathrm{E}-01 & 1.7 \mathrm{E}-01 & 1.8 \mathrm{E}-01 \\ \text { TOTAL } & 2.5 \mathrm{E}-01 & 2.4 \mathrm{E}-01 & 2.6 \mathrm{E}-01 & 2.5 \mathrm{E}-01 & 2.4 \mathrm{E}-01 & 2.5 \mathrm{E}-01\end{array}$

Production/Consumption factors:

Produce: $1.1 \quad$ Milk: $26 \quad$ Meat: 3.7

FRACTION OF POPULATION RECEIVING AN AVERAGE INDIVIOUAL TOTAL-BODY DOSE COMMITMENT FROM AIRBDRNE PATHWAYS

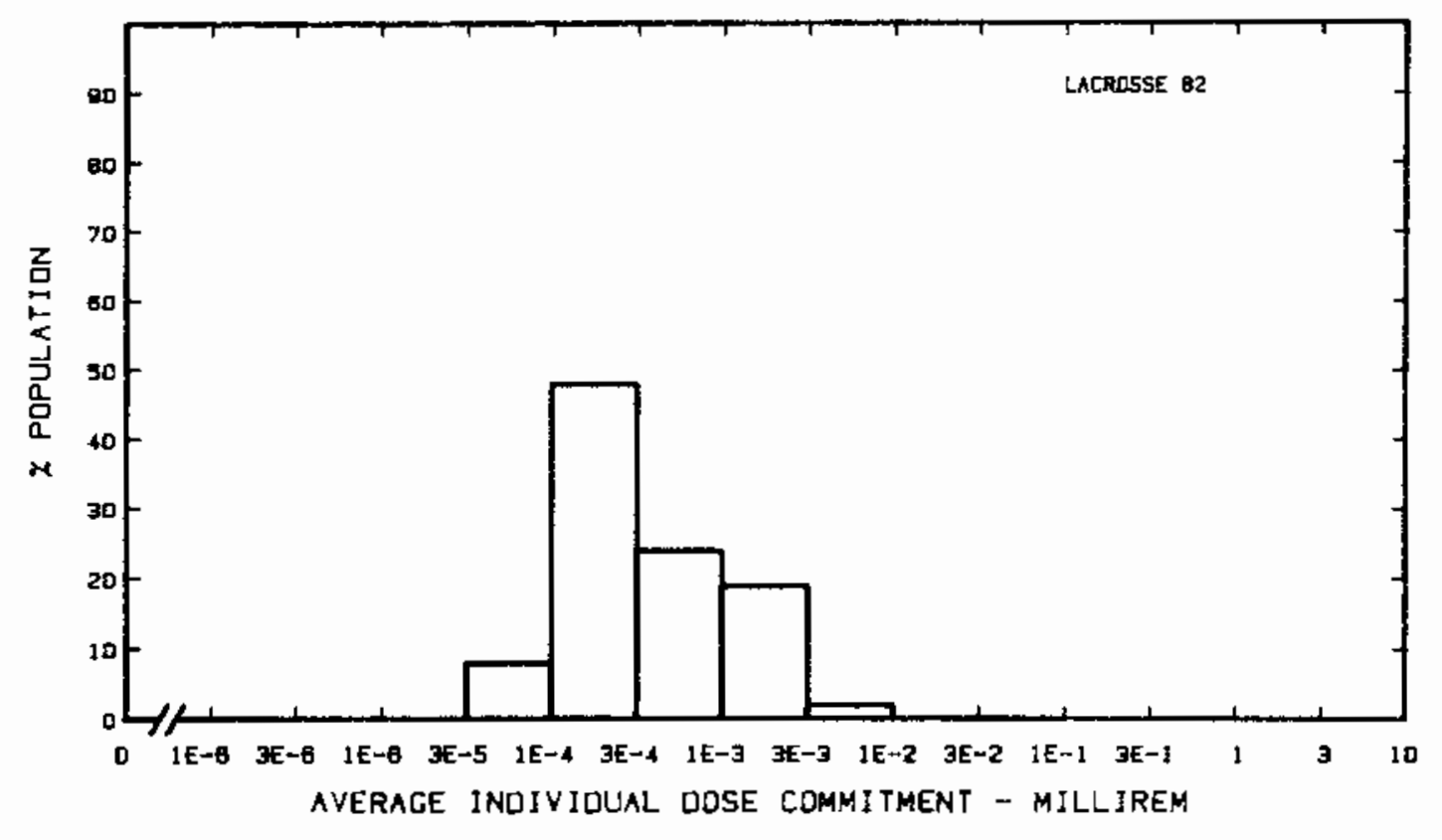


Site: LASALLE

SENECA, ILLINOIS

Location: N $41.2439^{\circ} \quad$ W $88.6708^{\circ}$

POPULATION DATA

Total Population Within 2-to-80-km Region: 1.0E6

Major Metropolitan Centers Within Region:

Center Population Location

Jol iet $\quad 78,000 \quad 59 \mathrm{~km} \quad$ NNE

Aurora $\quad 80,000 \quad 65 \mathrm{~km}$ NNE

Kankakee $\quad 103,000 \quad 69 \mathrm{~km}$ ESE

Dekalb $33,000 \quad 77 \mathrm{~km} \mathrm{~N}$

Naperville $\quad 42,000 \quad 73 \mathrm{~km} \quad \mathrm{NE}$

SITE SPECIFIC DATA - AIRBORNE PATHWAYS

Average Annual State Production

of Crops and Animal Products

In 80-km Radius Circle

Regional Productivity Factor:

Animal Grazing Factor:

Meteorology Period of Record: 1 Jan $82-31$ Dec 82 Recovery: $99 \%$
Veg: $1.1 E 8$ kilogram

Mi]k: 1.8E8 liter

Meat: $1.9 \mathrm{E} 8 \mathrm{kilogram}$

0.9

0.5

SITE SPECIFIC DATA - WATERBORNE PATHWAYS via ILLINOIS RIVER

Average River Flow

at site: $11,000 \mathrm{ft}^{3} / \mathrm{s}$

Drinking Water: Exposed Population: None

Dilution Factor:

Fish: Edible Harvest: None ${ }^{(a)}$

Dilution Factor:

(a) Because of river quality, no fish consumption is considered (FES 1973) 


\section{POPULATION DOSE-COMAITMENT ESTIMATES AMD AVERAGE INDIVIDUAL DOSE-COMIITMENT HISTOGRAM FOR}

LASALLE

Dose Commitments (person-rem) from Liquid Pathways

Total Body GI-LII Thyroid Bone Liver

Infant

Child

Teen

Adult

TOTAL

Dose Commitments (person-rem) from Airborne Pathways

Total Body GI-LII Thyroid Bone Liver Lung

$\begin{array}{lllllll}\text { Infant } & 7.8 \mathrm{E}-07 & 7.7 \mathrm{E}-07 & 6.9 \mathrm{E}-07 & 1.1 \mathrm{E}-06 & 6.9 \mathrm{E}-07 & 8.7 \mathrm{E}-07 \\ \text { Child } & 1.7 \mathrm{E}-05 & 1.2 \mathrm{E}-05 & 7.8 \mathrm{E}-06 & 4.4 \mathrm{E}-05 & 8.2 \mathrm{E}-06 & 1.1 \mathrm{E}-05 \\ \text { Teen } & 9.4 \mathrm{E}-06 & 7.5 \mathrm{E}-06 & 5.6 \mathrm{E}-06 & 2.2 \mathrm{E}-05 & 5.8 \mathrm{E}-06 & 8.4 \mathrm{E}-06 \\ \text { Adu1t } & 5.0 \mathrm{E}-05 & 4.3 \mathrm{E}-05 & 3.4 \mathrm{E}-05 & 1.1 \mathrm{E}-04 & 3.5 \mathrm{E}-05 & 4.4 \mathrm{E}-05 \\ \text { TOTAL } & 7.7 \mathrm{E}-05 & 6.1 \mathrm{E}-05 & 4.8 \mathrm{E}-05 & 1.7 \mathrm{E}-04 & 4.9 \mathrm{E}-05 & 6.4 \mathrm{E}-05\end{array}$

Production/Consumption factors:

Produce: $0.5 \quad$ Milk: $1.2 \quad$ Meat: 2.0

FRACTION OF PDPULATION RECEJVING AN AVERAGE INDIVIDUAL

TOTAL-BODY DOSE COMMJTMENT FROM AIREORNE PATHWAYS

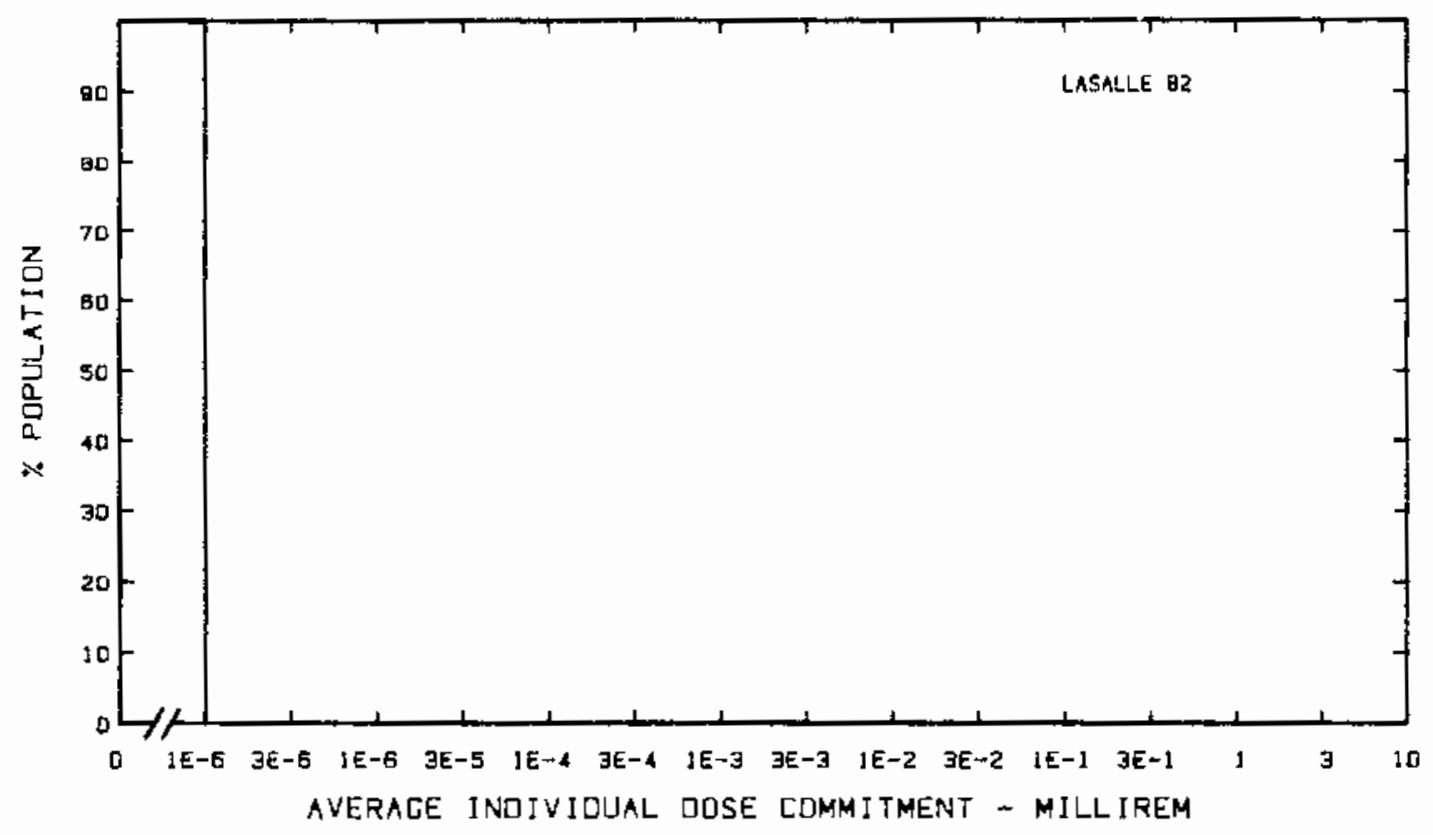


Site: MAINE YANKEE

LINCOLN COUNTY, MAINE

Location: N $\quad$ N $43.9506^{\circ}$ W $69.6961^{\circ}$

POPULATION OATA

Total Population Within 2-to-80-km Region: 5.9E5

Major Metropolitan Centers Within Region:

Center

Portland SMSA

Lewiston-Auburn SMSA

Augusta

Biddeford

Waterville
Population

180,000

72,000

22,000

20,000

18,000
Location

$56 \mathrm{~km} \quad W S W$

$45 \mathrm{~km} \quad \mathrm{WNW}$

$41 \mathrm{~km} \mathrm{~N}$

$80 \mathrm{~km} \quad \mathrm{SW}$

$67 \mathrm{~km} \quad \mathrm{~N}$

\section{SITE SPECIFIC DATA - AIRBORNE PATHWAYS}

Average Annual State Production

Of Crops and Animal Products

In 80-km Radius Circle

Regional Productivity Factor:

Animal Grazing Factor:

Meteorology Period of Record: 1 APR 75 - 31 MAR 76 Recovery: 98\%
Veg: $2.4 E 8$ kilogram

Mijk: $6.6 E 7$ liter

Meat: $4.3 \mathrm{E} 6 \mathrm{ki}$ logram

0.6

0.5

SITE SPECIFIC OATA - WATERBORNE PATHWAYS via ATLANTIC OCEAN

Average 0ilution Flow

from Plant: $707 \mathrm{ft}^{3} / \mathrm{s}$

Fish:

Edible Harvest: (a)

Dilution Factor: 0.001

Invertebrates:

Edible Harvest: (a)

Dilution Factor: 0.002

(a) No seafood harvest data given in FES (1972), thus generic population consumption rates used (Table A-1). 
POPULATION DOSE-COMMITMENT ESTIMATES ANO AVERAGE INDIVIDUAL OOSE-COMMITMENT HISTOGRAM FOR

\section{MAINE YANKEE}

Dose Commitments (person-rem) from Liquid Pathways

\begin{tabular}{|c|c|c|c|c|c|}
\hline & Total Body & GI-LLI & Thyroid & Bone & Liver \\
\hline $\begin{array}{l}\text { Infant } \\
\text { Child } \\
\text { Teen } \\
\text { Adult }\end{array}$ & $\begin{array}{l}0.0 \mathrm{E}+00 \\
4.3 \mathrm{E}-04 \\
3.3 \mathrm{E}-04 \\
2.3 \mathrm{E}-03\end{array}$ & $\begin{array}{l}0.0 \mathrm{E}+00 \\
7.5 \mathrm{E}-04 \\
1.6 \mathrm{E}-03 \\
1.4 \mathrm{E}-02\end{array}$ & $\begin{array}{l}0.0 \mathrm{E}+00 \\
8.2 \mathrm{E}-04 \\
5.8 \mathrm{E}-04 \\
3.8 \mathrm{E}-03\end{array}$ & $\begin{array}{l}0.0 \mathrm{E}+00 \\
1.9 \mathrm{E}-04 \\
1.1 \mathrm{E}-04 \\
6.6 \mathrm{E}-04\end{array}$ & $\begin{array}{l}0.0 E+00 \\
3.3 E-04 \\
2.8 E-04 \\
1.7 E-03\end{array}$ \\
\hline TOTAL & $3.0 \mathrm{E}-03$ & 1.7E-02 & $5.2 E-03$ & $9.7 \mathrm{E}-04$ & $2.4 \mathrm{E}-03$ \\
\hline
\end{tabular}

Dose Commitments (person-rem) from Airborne Pathways Total Body GI-LLI Thyrojd Bone Liver Lung

$\begin{array}{lllllll}\text { Infant } & 4.6 \mathrm{E}-05 & 4.6 \mathrm{E}-05 & 6.3 \mathrm{E}-05 & 3.2 \mathrm{E}-05 & 4.6 \mathrm{E}-05 & 4.9 \mathrm{E}-05 \\ \text { Child } & 7.7 \mathrm{E}-04 & 7.7 \mathrm{E}-04 & 1.0 \mathrm{E}-03 & 3.6 \mathrm{E}-04 & 7.7 \mathrm{E}-04 & 8.1 \mathrm{E}-04 \\ \text { Teen } & 5.0 \mathrm{E}-04 & 5.0 \mathrm{E}-04 & 6.1 \mathrm{E}-04 & 2.6 \mathrm{E}-04 & 5.0 \mathrm{E}-04 & 5.5 \mathrm{E}-04 \\ \text { Adult } & 2.8 \mathrm{E}-03 & 2.8 \mathrm{E}-03 & 3.3 \mathrm{E}-03 & 1.6 \mathrm{E}-03 & 2.8 \mathrm{E}-03 & 3.0 \mathrm{E}-03 \\ \text { TOTAL } & 4.2 \mathrm{E}-03 & 4.2 \mathrm{E}-03 & 4.9 \mathrm{E}-03 & 2.3 \mathrm{E}-03 & 4.2 \mathrm{E}-03 & 4.4 \mathrm{E}-03\end{array}$

Production/Consumption factors:

Produce: 1.3 Milk: $<1 \quad$ Meat: $<1$

FRACTION OF POPULATION RECEJVING AN AVERAGE INDIVIDUAL TOTAL-BODY ODSE COMMITMENT FROM AJRBDRNE PATHWAYS

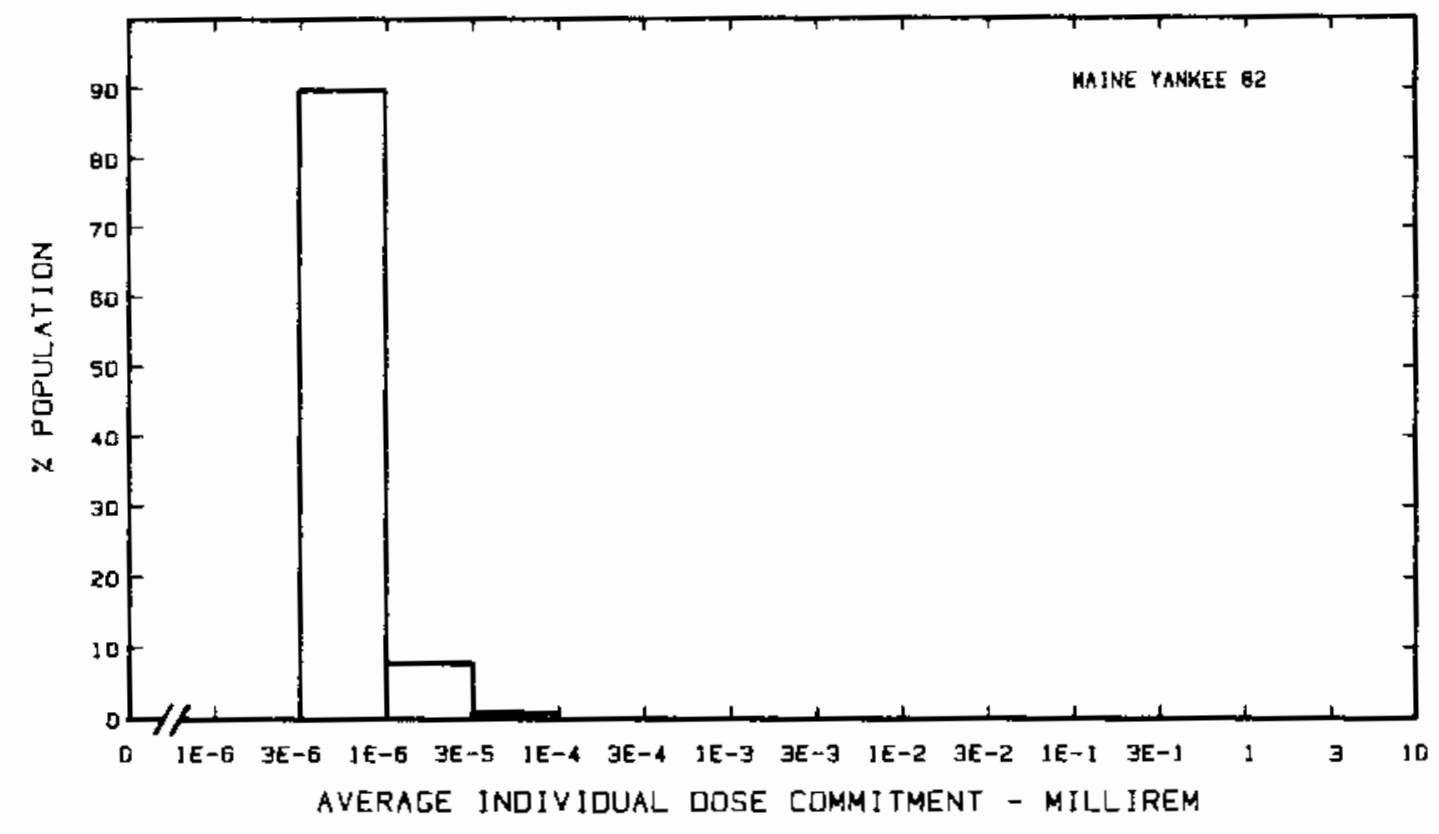


Site: MCGUIRE

CORNELIUS, NORTH CAROLINA

Location: $\quad$ N $35.4322^{\circ} \quad$ W $80.9483^{\circ}$

POPULATION OATA

Total Population Within 2-to-80-km Region: $1.6 \mathrm{E} 6$

Major Metropolitan Centers Within Region:

\begin{tabular}{|c|c|c|}
\hline Center & Population & Locat \\
\hline $\begin{array}{l}\text { Charlotte-Gastonia SMSA } \\
\text { Rockhill } \\
\text { Kannapolis } \\
\text { Salisbury } \\
\text { Hickory }\end{array}$ & $\begin{array}{r}640,000 \\
35,000 \\
35,000 \\
23,000 \\
21,000\end{array}$ & $\begin{array}{ll}25 & \mathrm{~km} \\
57 & \mathrm{~km} \\
30 & \mathrm{~km} \\
51 & \mathrm{~km} \\
49 & \mathrm{~km}\end{array}$ \\
\hline
\end{tabular}

SITE SPECIFIC DATA - AIRBORNE PATHWAYS

Average Annual State Production

of Crops and Animal Products

In 8D-km Radius Circle

Regional Productivity Factor:

Animal Grazing Factor:

Meteorology Period of Record: 17 OCT 70 - 16 OCT 71 Recovery: 90\%

SITE SPECIFIC DATA - WATERBORNE PATHWAYS via LAKE NORMAN On CATAWBA RIVER Average River

Flow at Site: $28,000 \mathrm{ft}^{3} / \mathrm{s}$

Drinking Water:

Exposed Population: 700,000

Fish:

Edible Harvest: (a) kq/yr

DiTution Factior: $0.5^{\text {pay }}$ 


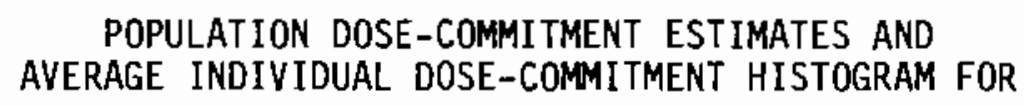

MCGUIRE

Dose Commitments (person-rem) from Liquid Pathways

Total Body GI-LLI $\underline{\text { Thyroid }}$ Bone

$\begin{array}{llllll}\text { Infant } & 4.4 \mathrm{E}-03 & 4.5 \mathrm{E}-03 & 3.9 \mathrm{E}-03 & 3.4 \mathrm{E}-04 & 4.0 \mathrm{E}-03 \\ \text { Child } & 5.2 \mathrm{E}-02 & 8.1 \mathrm{E}-02 & 4.2 \mathrm{E}-02 & 8.4 \mathrm{E}-03 & 4.8 \mathrm{E}-02 \\ \text { Teen } & 2.0 \mathrm{E}-02 & 8.0 \mathrm{E}-02 & 1.6 \mathrm{E}-02 & 3.5 \mathrm{E}-03 & 2.0 \mathrm{E}-02 \\ \text { Adult } & 1.6 \mathrm{E}-01 & 7.1 \mathrm{E}-01 & 1.3 \mathrm{E}-01 & 2.1 \mathrm{E}-02 & 1.6 \mathrm{E}-01 \\ \text { TOTAL } & 2.4 \mathrm{E}-01 & 8.8 \mathrm{E}-01 & 1.9 \mathrm{E}-01 & 3.3 \mathrm{E}-02 & 2.3 \mathrm{E}-01\end{array}$

Dose Commitments (person-rem) from Airborne Pathways

\begin{tabular}{|c|c|c|c|c|c|c|}
\hline & Total Body & GI-LLI & Thyroid & Bone & Liver & Lung \\
\hline $\begin{array}{l}\text { Infant } \\
\text { Child } \\
\text { Teen } \\
\text { Adult }\end{array}$ & $\begin{array}{l}2.2 \mathrm{E}-03 \\
2.4 \mathrm{E}-02 \\
1.8 \mathrm{E}-02 \\
1.1 \mathrm{E}-01\end{array}$ & $\begin{array}{l}2.2 \mathrm{E}-03 \\
2.4 \mathrm{E}-02 \\
1.8 \mathrm{E}-02 \\
1.1 \mathrm{E}-01\end{array}$ & $\begin{array}{l}2.2 \mathrm{E}-03 \\
2.4 \mathrm{E}-02 \\
1.8 \mathrm{E}-02 \\
1.1 \mathrm{E}-01\end{array}$ & $\begin{array}{l}2.2 \mathrm{E}-03 \\
2.4 \mathrm{E}-02 \\
1.7 \mathrm{E}-02 \\
1.1 \mathrm{E}-01\end{array}$ & $\begin{array}{l}2.2 \mathrm{E}-03 \\
2.4 \mathrm{E}-02 \\
1.8 \mathrm{E}-02 \\
1.1 \mathrm{E}-01\end{array}$ & $\begin{array}{l}2.2 \mathrm{E}-03 \\
2.5 \mathrm{E}-02 \\
1.9 \mathrm{E}-02 \\
1.1 \mathrm{E}-01\end{array}$ \\
\hline OTAL & 1. $5 \mathrm{E}-01$ & $1.5 \mathrm{E}-01$ & $1.5 \mathrm{E}-01$ & $1.5 \mathrm{E}-01$ & $1.5 \mathrm{E}-01$ & 1. $6 \mathrm{E}-01$ \\
\hline
\end{tabular}

Production/Consumption factors:

Produce: $<1 \quad$ Milk: $<1 \quad$ Meat: $<1$

FRACTION DF POPULATION RECEIVING AN AVERAGE INDIVIDUAL TOTAL-BODY DDSE COMMITMENT FROM AIRBORNE PATHWAYS

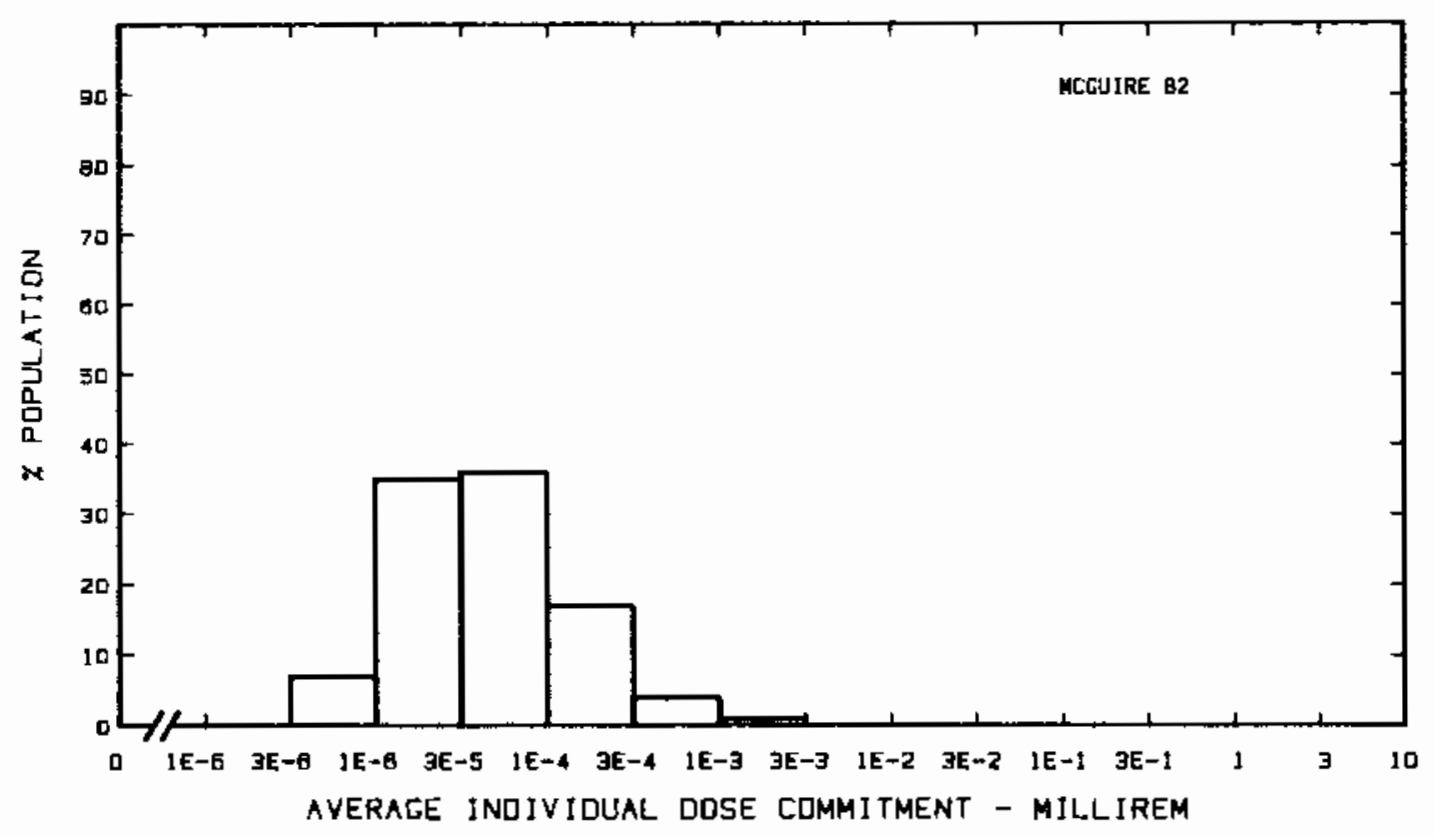


Site: MILLSTONE

WATERFORD, CONNECTICUT

Location: $\quad$ N $41.3086^{\circ} \quad$ W $72.1681^{\circ}$

POPULATION DATA

Total Population Within 2-to-80-km Region: 2.5E6

Major Metropolitan Centers Within Region:

Center

Hartford SMSA

New Haven-West Haven SMSA

New London-Norwich SMSA

New Britain SMSA

Waterbury SMSA
Population

730,000

420,000

250,000

140,000

230,000
Location

$67 \mathrm{~km} \mathrm{NW}$

$64 \mathrm{~km}$ W

$8 \mathrm{~km} \quad \mathrm{NNE}$

$65 \mathrm{~km} \quad \mathrm{NW}$

$78 \mathrm{~km} \quad W N W$

SITE SPECIFIC DATA - AIRBORNE PATHWAYS

Average Annual State Production

of Crops and Animal Products

In 80-km Radius Circle

Regional Productivity Factor:

Animal Grazing Factor:
Veg: $3.2 E 7$ kilogram

Milk: 4.4E8 Titer

Meat: $2.0 E 7$ kilogram

0.6

0.6

Meteorology Period of Record: 1 JAN $74-31$ OEC 74 Recovery: 95\%

SITE SPECIFIC OATA - WATERBORNE PATHWAYS via NIANTIC BAY

Average Dilution Flow

from Plant: $1,900 \mathrm{ft}^{3} / \mathrm{s}$

Fish:

Edible Harvest: $9.1 \mathrm{E} 4 \mathrm{~kg} / \mathrm{yr}$

Dilution Factor: 0.001

Invertebrates:

Edible Harvest: $9.1 \mathrm{E} 4 \mathrm{~kg} / \mathrm{yr}$

Dilution Factor: 0.002 
POPULATION DOSE-COMMITMENT ESTIMATES AND

AVERAGE INDIVIDUAL DOSE-COMMITMENT HISTOGRAM FOR

MILLSTONE 1 AND 2

Oose Commitments (person-rem) from Liquid Pathways

Total Body GI-LLI Thyroid Bone Liver

$\begin{array}{llllll}\text { Infant } & 0.0 \mathrm{E}+00 & 0.0 \mathrm{E}+00 & 0.0 \mathrm{E}+00 & 0.0 \mathrm{E}+00 & 0.0 \mathrm{E}+00 \\ \text { Child } & 5.1 \mathrm{E}-03 & 2.1 \mathrm{E}-02 & 7.9 \mathrm{E}-02 & 1.8 \mathrm{E}-02 & 2.2 \mathrm{E}-02 \\ \text { Teen } & 7.8 \mathrm{E}-03 & 4.5 \mathrm{E}-02 & 5.7 \mathrm{E}-02 & 1.1 \mathrm{E}-02 & 1.8 \mathrm{E}-02 \\ \text { Adult } & 7.9 \mathrm{E}-02 & 3.9 \mathrm{E}-01 & 3.7 \mathrm{E}-01 & 6.3 \mathrm{E}-02 & 1.1 \mathrm{E}-01 \\ \text { T0TAL } & 9.2 \mathrm{E}-02 & 4.6 \mathrm{E}-01 & 5.1 \mathrm{E}-01 & 9.2 \mathrm{E}-02 & 1.5 \mathrm{E}-01\end{array}$

Oose Commitments (person-rem) from Airborne Pathways Total Body GI-LLI Thyroid Bone Liver Lung

$\begin{array}{lllllll}\text { Infant } & 2.4 \mathrm{E}-02 & 2.4 \mathrm{E}-02 & 3.1 \mathrm{E}-01 & 2.5 \mathrm{E}-02 & 2.5 \mathrm{E}-02 & 2.5 \mathrm{E}-02 \\ \text { Child } & 2.7 \mathrm{E}-01 & 2.7 \mathrm{E}-01 & 1.8 \mathrm{E}+00 & 2.7 \mathrm{E}-01 & 2.7 \mathrm{E}-01 & 2.8 \mathrm{E}-01 \\ \text { Teen } & 2.0 \mathrm{E}-01 & 2.0 \mathrm{E}-01 & 7.9 \mathrm{E}+01 & 1.9 \mathrm{E}-01 & 2.0 \mathrm{E}-01 & 2.1 \mathrm{E}-01 \\ \text { Adult } & 1.2 \mathrm{E}+00 & 1.2 \mathrm{E}+00 & 3.1 \mathrm{E}+00 & 1.1 \mathrm{E}+00 & 1.2 \mathrm{E}+00 & 1.2 \mathrm{E}+00 \\ \text { T0TAL } & 1.7 \mathrm{E}+00 & 1.7 \mathrm{E}+00 & 6.0 \mathrm{E}+00 & 1.6 \mathrm{E}+00 & 1.7 \mathrm{E}+00 & 1.8 \mathrm{E}+00\end{array}$

Production/Consumption factors:

Produce: $<1 \quad$ Milk: <1 Meat: $<1$

FRACTION OF POPULATION RECEIVING AN AVERAGE INOIVIDUAL TOTAL-OOOY DOSE COMMITMENT FROM AIRBORNE PATHWAYS

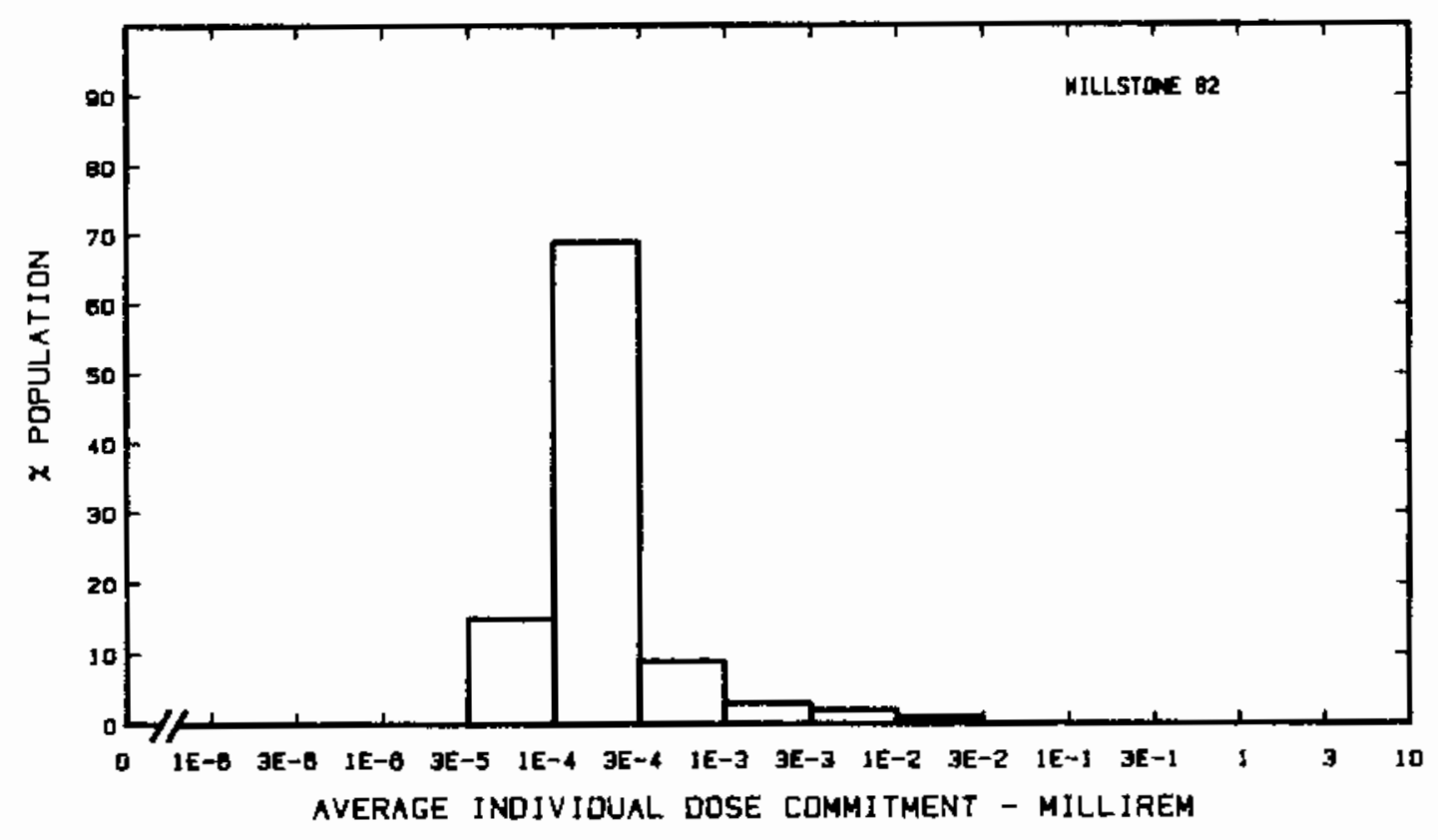


Site: MONTICELLO

Location: N $45.3333^{\circ}$
MONTICELLO, MINNESOTA

W $93.8483^{\circ}$

POPULATION DATA

Total Population Within 2-to-80-km Region: 2.1E6

Major Metropolitan Centers Within Region:

Center Population Location

Minneapolis-St. Paul SMSA $2,100,000 \quad 60 \mathrm{~km} \quad$ SE

St. Cloud SMSA $\quad 160,000 \quad 36 \mathrm{~km}$ NW

Bloomington $\quad 82,000 \quad 72 \mathrm{~km}$ SE

Edina $\quad 46,000 \quad 63 \mathrm{~km}$ SE

$\begin{array}{llll}\text { Richfield } & 38,000 & 67 \mathrm{~km} & \text { SE }\end{array}$

SITE SPECIFIC DATA - AIRBORNE PATHWAYS

Average Annual State Production

of Crops and Animal Products

In 80-km Radius Circle

Regional Productivity Factor:

Animal Grazing Factor:

Meteorology Period of Record: 1 JAN 74 - 31 DEC 74 Recovery: 92\%
Yeg: $1.2 \mathrm{E} 8 \mathrm{kilogram}$

Mi1k: 4.0E8 1iter

Meat: $1.1 \mathrm{E} 8$ kilogram

1

0.5

SITE SPECIFIC DATA - WATERBORNE PATHWAYS via MISSISSIPPI RIVER

Average River Flow

at Site: $4,600 \mathrm{ft}^{3} / \mathrm{s}$

Drinking Water:

Exposed Population: $1,800,000$

Fish:

Edible Harvest: $2.4 \mathrm{E} 5 \mathrm{~kg} / \mathrm{yr}$ 
POPULATION DOSE-COMMITMENT ESTIMATES AND

AVERAGE INDIVIDUAL OOSE-COMMITMENT HISTOGRAM FOR

MONTICELLO

Dose Commitments (person-rem) from Liquid Pathways

\begin{tabular}{|c|c|c|c|c|c|}
\hline & Total Body & GI-LLI & Thyroid & Bone & Liver \\
\hline $\begin{array}{l}\text { Infant } \\
\text { Child } \\
\text { Teen } \\
\text { Adult }\end{array}$ & $\begin{array}{l}2.5 E-08 \\
2.7 E-07 \\
8.9 E-08 \\
6.5 E-07\end{array}$ & $\begin{array}{l}2.5 E-08 \\
4.2 E-07 \\
3.3 E-07 \\
3.1 E-06\end{array}$ & $\begin{array}{l}9.0 \mathrm{E}-09 \\
1.0 \mathrm{E}-07 \\
3.9 \mathrm{E}-08 \\
3.3 \mathrm{E}-07\end{array}$ & $\begin{array}{l}0.0 \mathrm{E}+00 \\
0.0 \mathrm{E}+00 \\
0.0 \mathrm{E}+00 \\
0.0 \mathrm{E}+00\end{array}$ & $\begin{array}{l}1.6 \mathrm{E}-08 \\
1.6 \mathrm{E}-07 \\
6.1 \mathrm{E}-08 \\
4.8 \mathrm{E}-07\end{array}$ \\
\hline AL & $1.0 \mathrm{E}-06$ & 3. $9 \mathrm{E}-06$ & 4. $8 \mathrm{E}-07$ & $0.0 \mathrm{E}+00$ & $7.1 E-1$ \\
\hline
\end{tabular}

Dose Commitments (person-rem) from Airborne Pathways

Total Body GI-LLI Thyroid Bone Liver Lung

$\begin{array}{lllllll}\text { Infant } & 2.8 \mathrm{E}-03 & 2.6 \mathrm{E}-03 & 9.8 \mathrm{E}-02 & 2.9 \mathrm{E}-03 & 3.3 \mathrm{E}-03 & 2.8 \mathrm{E}-03 \\ \text { ChiTd } & 3.4 \mathrm{E}-02 & 3.1 \mathrm{E}-02 & 6.6 \mathrm{E}-01 & 3.8 \mathrm{E}-02 & 3.6 \mathrm{E}-02 & 3.3 \mathrm{E}-02 \\ \text { Teen } & 2.3 \mathrm{E}-02 & 2.2 \mathrm{E}-02 & 2.6 \mathrm{E}-01 & 2.3 \mathrm{E}-02 & 2.4 \mathrm{E}-02 & 2.4 \mathrm{E}-02 \\ \text { Adult } & 1.3 \mathrm{E}-01 & 1.3 \mathrm{E}-01 & 9.3 \mathrm{E}-01 & 1.3 \mathrm{E}-01 & 1.3 \mathrm{E}-01 & 1.4 \mathrm{E}-01 \\ \text { TOTAL } & 1.9 \mathrm{E}-01 & 1.9 \mathrm{E}-01 & 1.9 \mathrm{E}-00 & 1.9 \mathrm{E}-01 & 2.0 \mathrm{E}-01 & 2.0 \mathrm{E}-01\end{array}$

Production/Consumption factors:

Produce: $<1 \quad$ Milk: $1.4 \quad$ Meat: $<1$

FRACTIDN OF POPULATION RECEIVING AN AVERAGE INDIVIDUAL TOTAL-BODY ODSE COMMITMENT FRDM AIRBORNE PATHYAYS

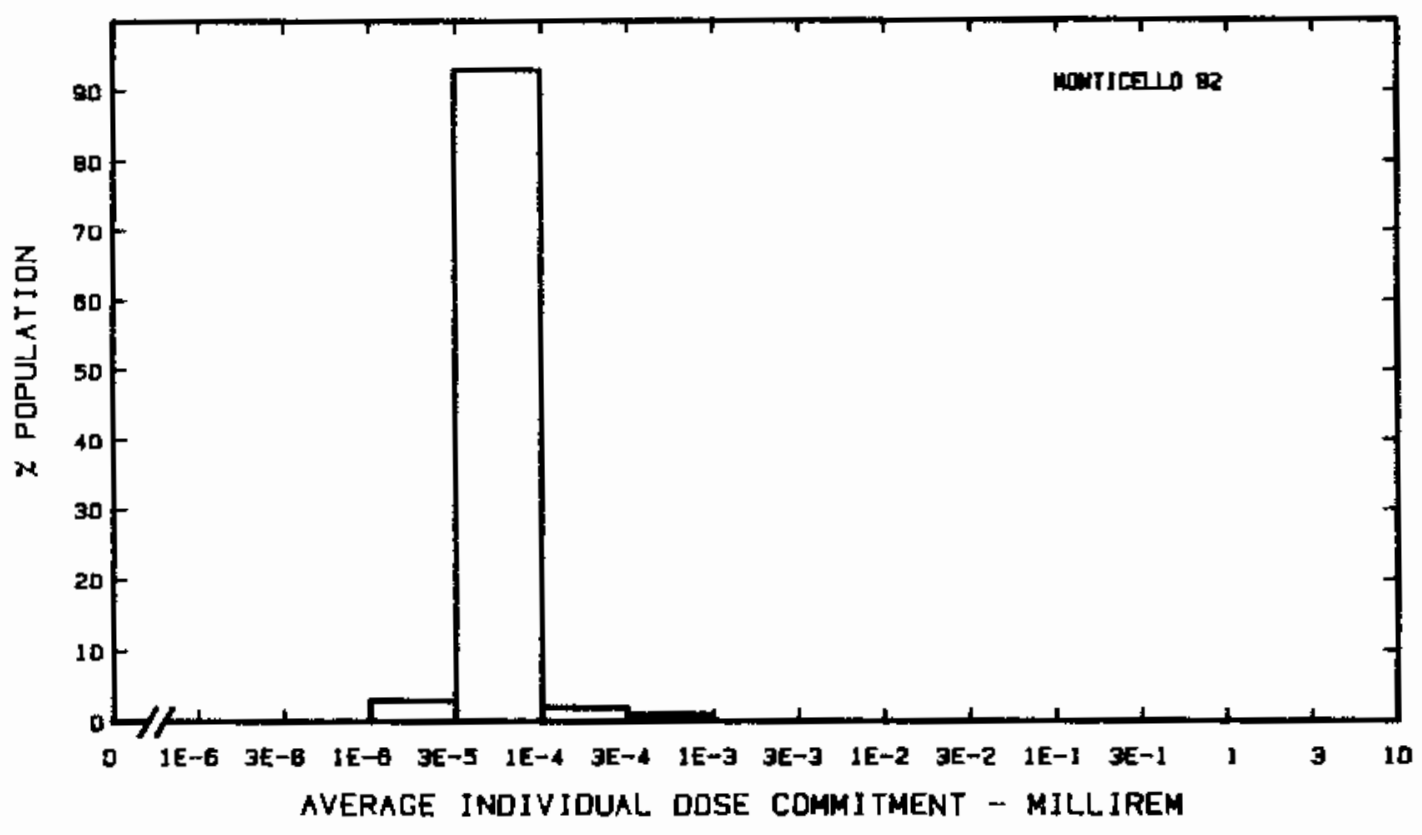


Site: NINE MILE POINT

Location: N $43.5222^{\circ}$ W $76.4100^{\circ}$

POPULATION DATA

Total Population Within 2-to-80-km Region: $8.4 \mathrm{E5}$

Major Metropolitan Centers Within Region:

\begin{tabular}{|c|c|c|c|}
\hline Center & Population & \multicolumn{2}{|c|}{ Location } \\
\hline Syracuse SMSA & 640,000 & $56 \mathrm{~km}$ & SSE \\
\hline Auburn & 33,000 & $67 \mathrm{~km}$ & SSW \\
\hline Watertown & 28,000 & $64 \mathrm{~km}$ & $\mathrm{NE}$ \\
\hline Kingston & 24,000 & $79 \mathrm{~km}$ & N \\
\hline Oswego & 20,000 & $11 \mathrm{~km}$ & SW \\
\hline
\end{tabular}

SITE SPECIFIC DATA - AIRBORNE PATHWAYS

Average Annual State Production

of Crops and Animal Products

In 80-km Radius Circle

Regional Productivity Factor:

Animal Grazing Factor:

Meteorology Period of Record: 1 JAN 74 - 31 DEC 75 Recovery: 97\%
Veg: $7.6 \mathrm{E} 7 \mathrm{ki}$ iogram

Milk: 7.0E8 liter

0.7

0.5
Meat: $3.3 \mathrm{E} 7$ kilogram

SITE SPECIFIC OATA - WATERBORNE PATHWAYS via LAKE ONTARIO

Average Dilution Flow

from Plant: $120 \mathrm{ft}^{3} / \mathrm{s}$

Drinking Water:

Exposed Population: 550,000

Dilution Factor: 0.01

Fish:

Edible Harvest: $7.3 \mathrm{E} 5 \mathrm{~kg} / \mathrm{yr}$

Dilution Factor: 0.0033 


\section{POPULATION OOSE-COMMITMENT ESTIMATES ANO}

AVERAGE INOIVIOUAL DOSE-COMMITMENT HISTOGRAM FOR

NINE MILE POINT

Dose Commitments (person-rem) from Liquid Pathways

\begin{tabular}{|c|c|c|c|c|c|}
\hline & Total Body & GI-LLI & Thyroid & Bone & Liver \\
\hline $\begin{array}{l}\text { Infant } \\
\text { Child } \\
\text { Teen } \\
\text { Adult }\end{array}$ & $\begin{array}{l}2.3 \mathrm{E}-04 \\
2.7 \mathrm{E}-03 \\
1.1 \mathrm{E}-03 \\
9.8 \mathrm{E}-03\end{array}$ & $\begin{array}{l}2.3 \mathrm{E}-04 \\
2.6 \mathrm{E}-03 \\
1.1 \mathrm{E}-03 \\
9.4 \mathrm{E}-03\end{array}$ & $\begin{array}{l}2.2 \mathrm{E}-04 \\
2.5 \mathrm{E}-03 \\
9.5 \mathrm{E}-04 \\
8.1 \mathrm{E}-03\end{array}$ & $\begin{array}{l}4.2 \mathrm{E}-05 \\
8.5 \mathrm{E}-04 \\
3.5 \mathrm{E}-04 \\
2.2 \mathrm{E}-03\end{array}$ & $\begin{array}{l}2.5 \mathrm{E}-04 \\
3.1 \mathrm{E}-03 \\
1.3 \mathrm{E}-03 \\
1.0 \mathrm{E}-02\end{array}$ \\
\hline TOTAL & $1.4 \mathrm{E}-02$ & 1. $3 \mathrm{E}-02$ & 1.2E-02 & $3.5 \mathrm{E}-03$ & $1.5 \mathrm{E}-02$ \\
\hline
\end{tabular}

Dose Commitments (person-rem) from Airborne Pathways

\begin{tabular}{|c|c|c|c|c|c|c|}
\hline & Total Body & GI-LLI & Thyroid & Bone & Liver & Lung \\
\hline $\begin{array}{l}\text { Infant } \\
\text { Child } \\
\text { Teen } \\
\text { Adult }\end{array}$ & $\begin{array}{l}1.9 \mathrm{E}-04 \\
2.8 \mathrm{E}-03 \\
1.7 \mathrm{E}-03 \\
9.2 \mathrm{E}-03\end{array}$ & $\begin{array}{l}1.8 \mathrm{E}-04 \\
2.6 \mathrm{E}-03 \\
1.7 \mathrm{E}-03 \\
9.2 \mathrm{E}-03\end{array}$ & $\begin{array}{l}1.5 \mathrm{E}-03 \\
1.1 \mathrm{E}-02 \\
4.7 \mathrm{E}-03 \\
1.8 \mathrm{E}-02\end{array}$ & $\begin{array}{l}1.7 \mathrm{E}-04 \\
2.6 \mathrm{E}-03 \\
1.3 \mathrm{E}-03 \\
6.2 \mathrm{E}-03\end{array}$ & $\begin{array}{l}2.8 \mathrm{E}-04 \\
3.2 \mathrm{E}-03 \\
1.8 \mathrm{E}-03 \\
9.0 \mathrm{E}-03\end{array}$ & $\begin{array}{l}2.1 \mathrm{E}-04 \\
2.8 \mathrm{E}-03 \\
1.8 \mathrm{E}-03 \\
9.3 \mathrm{E}-03\end{array}$ \\
\hline TOTAL & $1.4 \mathrm{E}-02$ & $1.4 \mathrm{E}-02$ & $3.5 \mathrm{E}-02$ & 1.0E-02 & $1.4 \mathrm{E}-02$ & $1.4 \mathrm{E}-02$ \\
\hline
\end{tabular}

Production/Consumption factors:

Produce: $<1 \quad$ Milk: $4.4 \quad$ Meat: <1

FRACTION OF POPULATION RECEIVING AN AVERAGE INDIVIDUAL TDTAL-BDOY DOSE COMMITMENT FROM AIRBDRNE PATHWAYS

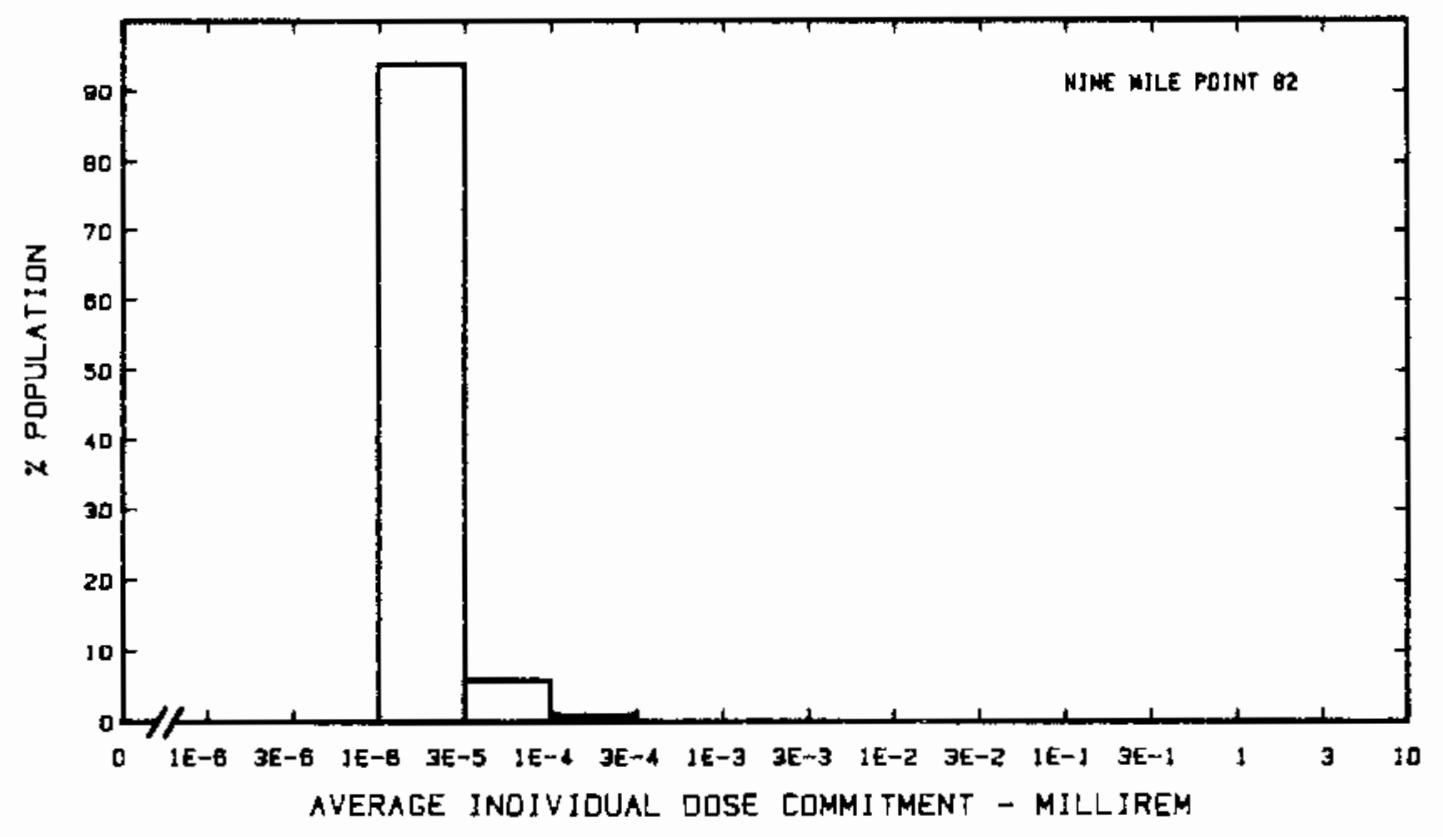


Site: NORTH ANNA

LOUISA COUNTY, VIRGINIA

Location: $\quad$ N $38.0608^{\circ}$ W $77.7906^{\circ}$

POPULATION DATA

Total Population Within 2-to-80-km Region: 1.0E6

Major Metropolitan Centers Within Region:

Center

Richmond SMSA

Charlottesville

Fredricksburg

Culpeper

Ashland
Population

630,000

40,000

15,000

6,600

4,600
Location

$66 \mathrm{~km} \quad$ SSE

$63 \mathrm{~km} \quad \mathrm{~W}$

$40 \mathrm{~km} \quad \mathrm{NE}$

$54 \mathrm{~km} \quad \mathrm{NNW}$

$41 \mathrm{~km} \quad \mathrm{SE}$

\section{SITE SPECIFIC DATA - AIRBORNE PATHWAYS}

Average Annual State Production

of Crops and Animal Products

In 80-km Radius Circle

Regional Productivity Factor:

Animal Grazing Factor:
Veg: $3.5 \mathrm{E} 7 \mathrm{kilogram}$

Mi 1k: 1.5E8 liter

Meat: $7.4 \mathrm{E} 7 \mathrm{kilogram}$

0.9

0.7

Meteorology Period of Record: 1 APR 74 - 3.L APR 75 Recovery: 99\%

SITE SPECIFIC DATA - WATERBORNE PATHWAYS via LAKE ANNA ${ }^{(a)}$

Average Dilution Flow from

Plant: $1,600 \mathrm{ft}^{3} / \mathrm{s}$

Drinking Water:

Exposed Population: None

Fish:

Edible Harvest: $7.3^{(\mathrm{b})}$
Dilution Factor: 0.001

(a) Reconcentration of radionuclides in lake accounted for (FES, 1973).

(b) Average individual consumption rates as given in the FES (1973) were used in lieu of catch data. 
Dose Commitments (person-rem) from Liquid Pathways

\begin{tabular}{|c|c|c|c|c|c|}
\hline & Total Body & GI-LLI & Thyroid & Bone & Liver \\
\hline $\begin{array}{l}\text { Infant } \\
\text { Child } \\
\text { Teen } \\
\text { Adult }\end{array}$ & $\begin{array}{l}0.0 \mathrm{E}+00 \\
2.2 \mathrm{E}-01 \\
4.2 \mathrm{E}-01 \\
4.6 \mathrm{E}+00\end{array}$ & $\begin{array}{l}0.0 \mathrm{E}+00 \\
1.4 \mathrm{E}-02 \\
2.7 \mathrm{E}-02 \\
2.3 \mathrm{E}-01\end{array}$ & $\begin{array}{l}0.0 \mathrm{E}+00 \\
4.1 \mathrm{E}-03 \\
3.1 \mathrm{E}-03 \\
2.1 \mathrm{E}-02\end{array}$ & $\begin{array}{l}0.0 \mathrm{E}+00 \\
1.1 \mathrm{E}+00 \\
6.8 \mathrm{E}-01 \\
4.0 \mathrm{E}+00\end{array}$ & $\begin{array}{l}0.0 \mathrm{E}+00 \\
1.3 \mathrm{E}+00 \\
1.1 \mathrm{E}+00 \\
6.4 \mathrm{E}+00\end{array}$ \\
\hline TOTAL & $5.2 E+00$ & $2.7 \mathrm{E}-01$ & $2.8 \mathrm{E}-02$ & $5.8 \mathrm{E}+00$ & $8.8 E+00$ \\
\hline
\end{tabular}

Dose Commitments (person-rem) from Airborne Pathways Total Body GI-LL1 Thyroid Bone Liver Lung

$\begin{array}{lllllll}\text { Infant } & 5.9 \mathrm{E}-04 & 5.6 \mathrm{E}-04 & 2.0 \mathrm{E}-02 & 6.4 \mathrm{E}-04 & 6.9 \mathrm{E}-04 & 6.1 \mathrm{E}-04 \\ \text { Child } & 6.5 \mathrm{E}-03 & 6.3 \mathrm{E}-03 & 1.1 \mathrm{E}-01 & 6.7 \mathrm{E}-03 & 7.1 \mathrm{E}-03 & 7.0 \mathrm{E}-03 \\ \text { Teen } & 4.7 \mathrm{E}-03 & 4.5 \mathrm{E}-03 & 4.4 \mathrm{E}-02 & 4.5 \mathrm{E}-03 & 4.9 \mathrm{E}-03 & 5.4 \mathrm{E}-03 \\ \text { Adult } & 2.8 \mathrm{E}-02 & 2.7 \mathrm{E}-02 & 1.5 \mathrm{E}-01 & 2.7 \mathrm{E}-02 & 2.8 \mathrm{E}-02 & 3.0 \mathrm{E}-02 \\ \text { TOTAL } & 4.0 \mathrm{E}-02 & 3.9 \mathrm{E}-02 & 3.2 \mathrm{E}-01 & 3.9 \mathrm{E}-02 & 4.1 \mathrm{E}-02 & 4.3 \mathrm{E}-02\end{array}$

Production/Consumption factors:

Produce: <1 Mi]k: 1.0 Meat: <1

FRACTION OF POPULATION RECEIVING AN AVERAGE INOIVIDUAL TOTAL-OOOY DOSE COMMITMENT FROM AIRBDRNE PATHWAYS

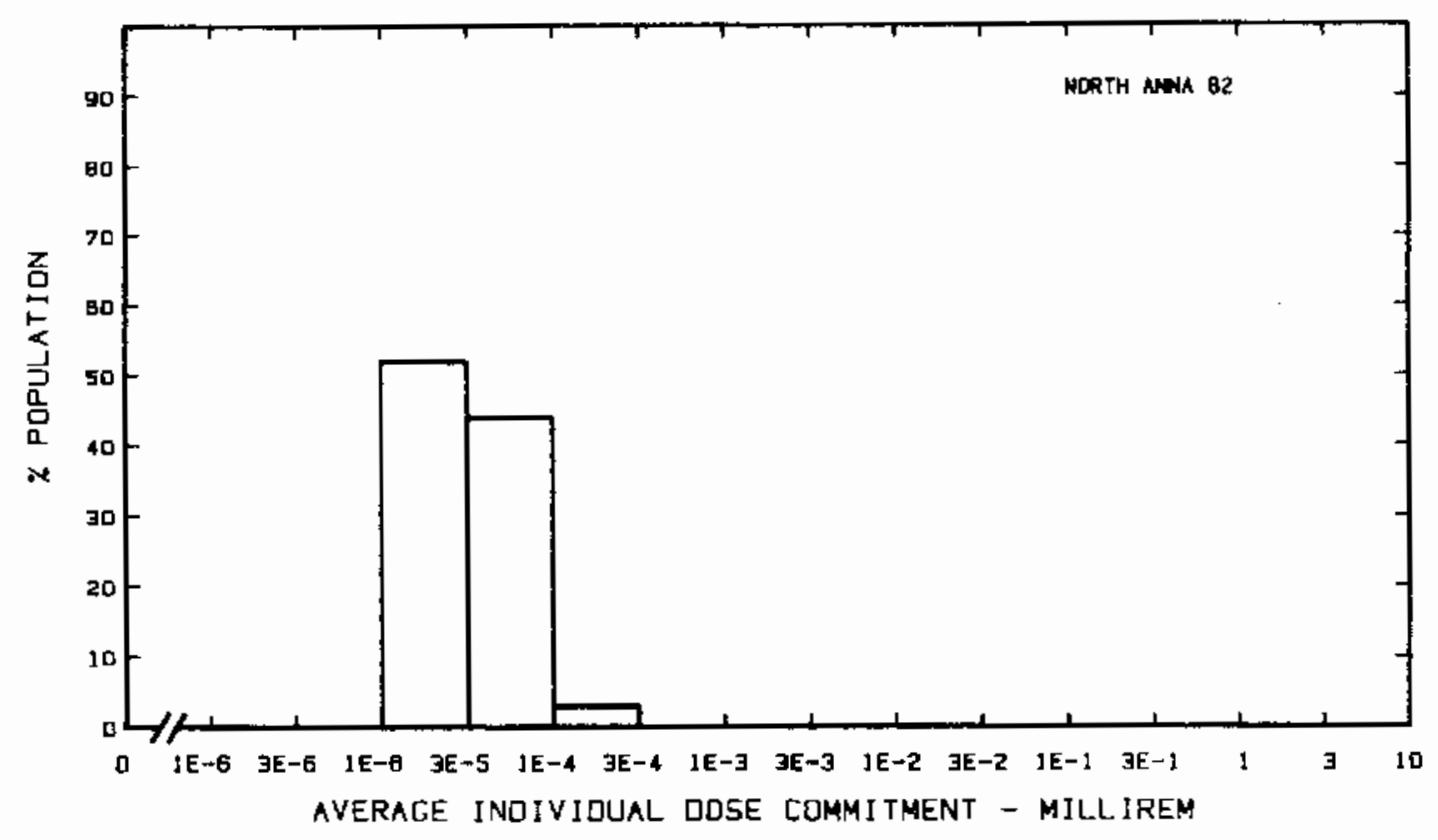


Site: OCONEE

OCONEE COUNTY, SOUTH CAROLINA

Location: N $34.7917^{\circ} \quad$ W $82.8986^{\circ}$

POPULATION DATA

Total Population Within 2-to-80-km Region: 9.0E5

Major Metropolitan Centers Within Region:

\begin{tabular}{lccc} 
Center & Population & \multicolumn{2}{c}{ Location } \\
\cline { 2 - 5 } Greenville SMSA & 560,000 & $46 \mathrm{~km}$ & $\mathrm{E}$ \\
Anderson & 27,000 & $39 \mathrm{~km}$ & SE \\
Easley & 14,000 & $27 \mathrm{~km}$ & E \\
Greer & 11,000 & $64 \mathrm{~km}$ & ENE
\end{tabular}

\section{SITE SPECIFIC DATA - AIRBORNE PATHWAYS}

Average Annual State Production

of Crops and Animal Products

In 80-km Radius Circle

Regional Productivity Factor:

Animal Grazing Factor:
Veg: $7.4 \mathrm{E} 6$ kilogram

Milk: 5.7E7 liter

Meat: $5.0 \mathrm{E} 7 \mathrm{kilogram}$

1

0.7

Meteorology Period of Record: 1 JAN 75 - 31 DEC 75 Recovery: 86\%

SITE SPECIFIC DATA - WATERBORNE PATHWAYS via HARTWELL RES. On KEOWEE RIVER

Average River Flow

at Site: $1,100 \mathrm{ft}^{3} / \mathrm{s}$

Drinking Water:

Exposed Population: 53,D00

Dilution Factor: 1

Fish:

Edible Harvest: (a)

Dilution Factor: $0.01^{\text {(b) }}$

(a) No fish catch data given in FES, so generic consumption rates used (Table A-1).

(b) Ten percent of population obtain $10 \%$ of their fish diet from Hartwell Reservoir (FES, 1972). 


\section{POPULATION DOSE-COMITMENT ESTIMATES AND AVERAGE INDIVIDUAL DOSE-COMMITMENT HISTOGRAM FOR}

OCONEE 1,2 AND 3

Dose Commitments (person-rem) from Liquid Pathways

\begin{tabular}{|c|c|c|c|c|c|}
\hline & Total Body & GI-LII & Thyroid & Bone & Liver \\
\hline $\begin{array}{l}\text { Infant } \\
\text { Child } \\
\text { Teen } \\
\text { Adult }\end{array}$ & $\begin{array}{l}2.5 \mathrm{E}-02 \\
6.4 \mathrm{E}-01 \\
7.5 \mathrm{E}-01 \\
8.0 \mathrm{E}+00\end{array}$ & $\begin{array}{l}1.6 \mathrm{E}-02 \\
2.0 \mathrm{E}-01 \\
1.1 \mathrm{E}-01 \\
9.0 \mathrm{E}-01\end{array}$ & $\begin{array}{l}3.9 \mathrm{E}-02 \\
3.3 \mathrm{E}-01 \\
1.2 \mathrm{E}-01 \\
9.1 \mathrm{E}-01\end{array}$ & $\begin{array}{l}6.9 \mathrm{E}-02 \\
2.3 \mathrm{E}+00 \\
1.1 \mathrm{E}+00 \\
6.8 \mathrm{E}+00\end{array}$ & $\begin{array}{l}\text { 8. } 1 \mathrm{E}-02 \\
2.4 \mathrm{E}+00 \\
1.7 \mathrm{E}+00 \\
1.0 \mathrm{E}+01\end{array}$ \\
\hline TOTAL & $9.4 \mathrm{E}+00$ & 1. $2 \mathrm{E}+00$ & 1. $4 \mathrm{E}+00$ & $1.0 \mathrm{E}+01$ & $1.5 \mathrm{E}+01$ \\
\hline
\end{tabular}

Dose Commitments (person-rem) from Airborne Pathways

Iotal Body GI-LII Thyroid Bone Liver $\underline{\text { Lung }}$

Infant

Child

$7.2 \mathrm{E}-03$

$7.1 \mathrm{E}-03$

8.0E-02

8.0E-02

7. $0 \mathrm{E}-02$

$7.8 \mathrm{E}-03$

4. $6 \mathrm{E}-01$

8.4E-D2

8.0E-03

8.0E-03

Teen

5. $9 \mathrm{E}-02$

5. 9E-02

2. $2 \mathrm{E}-01$

5. $9 \mathrm{E}-\mathrm{D} 2$

8. $5 \mathrm{E}-02$

9. $1 \mathrm{E}-02$

Adult

3. $6 \mathrm{E}-01$

3. $6 \mathrm{E}-01$

9. $7 \mathrm{E}-01$

3. 5E-01

6. 1E-D2

7. $2 \mathrm{E}-02$

TOTAL

5. OE-01

5. 1E-01

1. $7 \mathrm{E}+00$

5. OE-0I

3. $6 \mathrm{E}-01$

4. $0 \mathrm{E}-01$

5. 1E-01

5.7E-01

Production/Consumption factors:

Produce: $<1$

Milk: <1

Meat: $<1$

FRACTION OF POPULATION RECEIVING AN AVERACE INOIVIOUAL TUTAL-EODY DOSE COMMITMENT FROM AIREORNE PATHWAYS

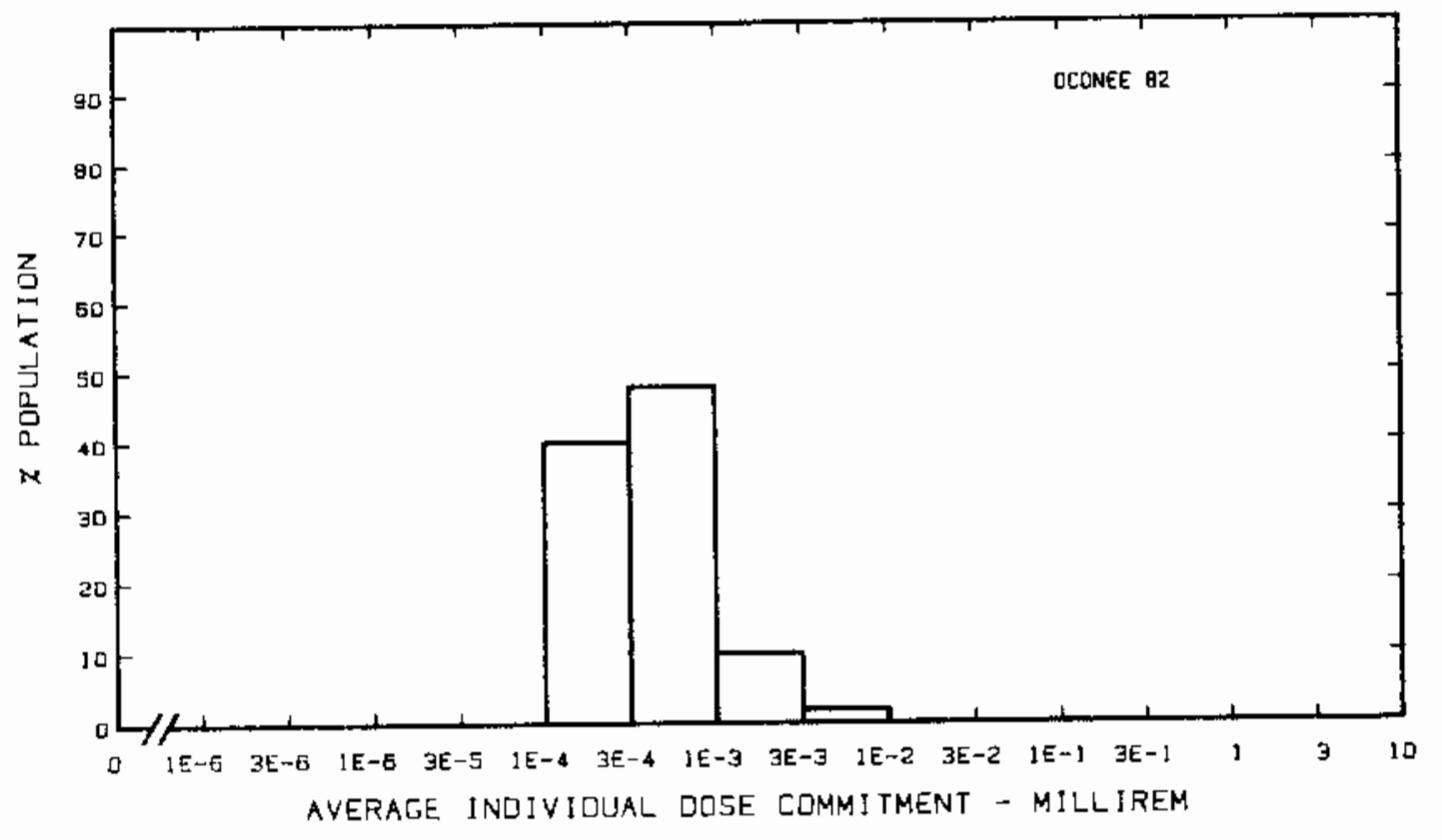


Site: OYSTER CREEK

OYSTER CREEK, NEW JERSEY

Location: $\quad N 39.8142^{\circ}$ W $74.2064^{\circ}$

POPULATION DATA

Total Population Within 2-to-80-km Region: 3.5E6

Major Metropolitan Centers Within Region:

Center Population Location

New Brunswick-Sayreville SMSA $600,000 \quad 77 \mathrm{~km} \quad \mathrm{~N}$

Long Branch-Asbury Park SMSA $500,000 \quad 57 \mathrm{~km}$ NNE

Trenton SMSA $\quad 310,000 \quad 66 \mathrm{~km} \quad \mathrm{NW}$

Atlantic City SMSA $\quad 190,000 \quad 55 \mathrm{~km} \quad$ SSW

Canden $\quad 85,000 \quad 79 \mathrm{~km} \quad W$

SITE SPECIFIC DATA - AIRBORNE PATHWAYS

Average Annual State Production

of Crops and Animal Products

In $80-\mathrm{km}$ Radius Circle

Regional Productivity Factor:

Animal Grazing Factor:
Veg: 7.4E7 kilogram

Milk: 2.7E8 liter

Meat: $2.4 E 7$ kilogram

0.5

0.6

Meteorology Period of Record: 15 FEB 66 - 31 DEC 68 Recovery: 63\%

SITE SPECIFIC DATA - WATERBORNE PATHWAYS via BARNEGAT BAY

Average Dilution Flow

from Plant: $1,500 \mathrm{ft}^{3} / \mathrm{s}$

Fish:

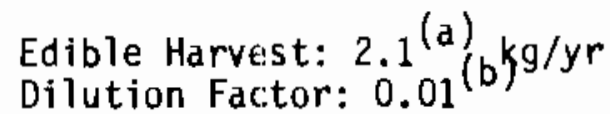

Invertebrates:

Edible Harvest: $0.96(\mathrm{a})$
Dilution Factor: $0.01(\mathrm{~b})^{\mathrm{kg} / \mathrm{yr}}$

(a) Average individual consumption rate as given in the FES (1974) used in lieu of catch data.

(b) $10 \%$ of seafood eaten assumed caught in bay waters diluted to $10 \%$ of that of discharge canal (FES, 1974). 
POPULATION DOSE-COMMITMENT ESTIMATES ANO

AVERAGE INDIVIDUAL DOSE-COMMITMENT HISTOGRAM FOR

OYSTER CREEK

Dose Commitments (person-rem) from Liquid Pathways

\begin{tabular}{|c|c|c|c|c|c|}
\hline & Total Body & GI-LII & Thyroid & Bone & Liver \\
\hline $\begin{array}{l}\text { Infant } \\
\text { Child } \\
\text { Teen } \\
\text { Adult }\end{array}$ & $\begin{array}{l}0.0 \mathrm{E}+00 \\
1.9 \mathrm{E}-03 \\
1.3 \mathrm{E}-03 \\
7.9 \mathrm{E}-03\end{array}$ & $\begin{array}{l}0.0 \mathrm{E}+00 \\
3.7 \mathrm{E}-03 \\
7.8 \mathrm{E}-03 \\
6.9 \mathrm{E}-02\end{array}$ & $\begin{array}{l}0.0 \mathrm{E}+00 \\
4.3 \mathrm{E}-06 \\
3.8 \mathrm{E}-06 \\
3.1 \mathrm{E}-05\end{array}$ & $\begin{array}{l}0.0 \mathrm{E}+00 \\
6.9 \mathrm{E}-06 \\
4.0 \mathrm{E}-06 \\
2.3 \mathrm{E}-05\end{array}$ & $\begin{array}{l}0.0 \mathrm{E}+00 \\
9.5 \mathrm{E}-04 \\
8.7 \mathrm{E}-04 \\
5.4 \mathrm{E}-03\end{array}$ \\
\hline TOTAL & $1.1 \mathrm{E}-02$ & $8.1 E-02$ & $3.9 \mathrm{E}-05$ & $3.4 \mathrm{E}-05$ & $7.2 \mathrm{E}-03$ \\
\hline
\end{tabular}

Dose Commitments (person-rem) from Airborne Pathways

Total Body GI-LII Thyroid Bone Liver Lung

$\begin{array}{lllllll}\text { Infant } & 1.1 \mathrm{E}-01 & 1.1 \mathrm{E}-01 & 6.1 \mathrm{E}-01 & 1.1 \mathrm{E}-01 & 1.1 \mathrm{E}-01 & 1.1 \mathrm{E}-01 \\ \text { Child } & 1.2 \mathrm{E}+00 & 1.2 \mathrm{E}+00 & 5.0 \mathrm{E}+00 & 1.2 \mathrm{E}+00 & 1.2 \mathrm{E}+00 & 1.3 \mathrm{E}+00 \\ \text { Teen } & 8.9 \mathrm{E}-01 & 9.0 \mathrm{E}-01 & 2.7 \mathrm{E}+00 & 9.0 \mathrm{E}-01 & 9.0 \mathrm{E}-01 & 9.5 \mathrm{E}-01 \\ \text { Adult } & 5.4 \mathrm{E}+00 & 5.4 \mathrm{E}+00 & 1.3 \mathrm{E}+01 & 5.4 \mathrm{E}+00 & 5.4 \mathrm{E}+00 & 5.6 \mathrm{E}+00 \\ \text { TOTAL } & 7.6 \mathrm{E}+00 & 7.7 \mathrm{E}+00 & 2.1 \mathrm{E}+01 & 7.7 \mathrm{E}+00 & 7.7 \mathrm{E}+00 & 7.9 \mathrm{E}+00\end{array}$

Production/Consumption factors:

Produce: $<1 \quad$ Milk: $<1 \quad$ Meat: <1

FRACTION DF POPULATIDN RECEIVING AN AVERAGE INDIVIOUAL TOTAL $\leadsto$ BODY DOSE COMMITMENT FROM AIRBDRNE PATHYAYS

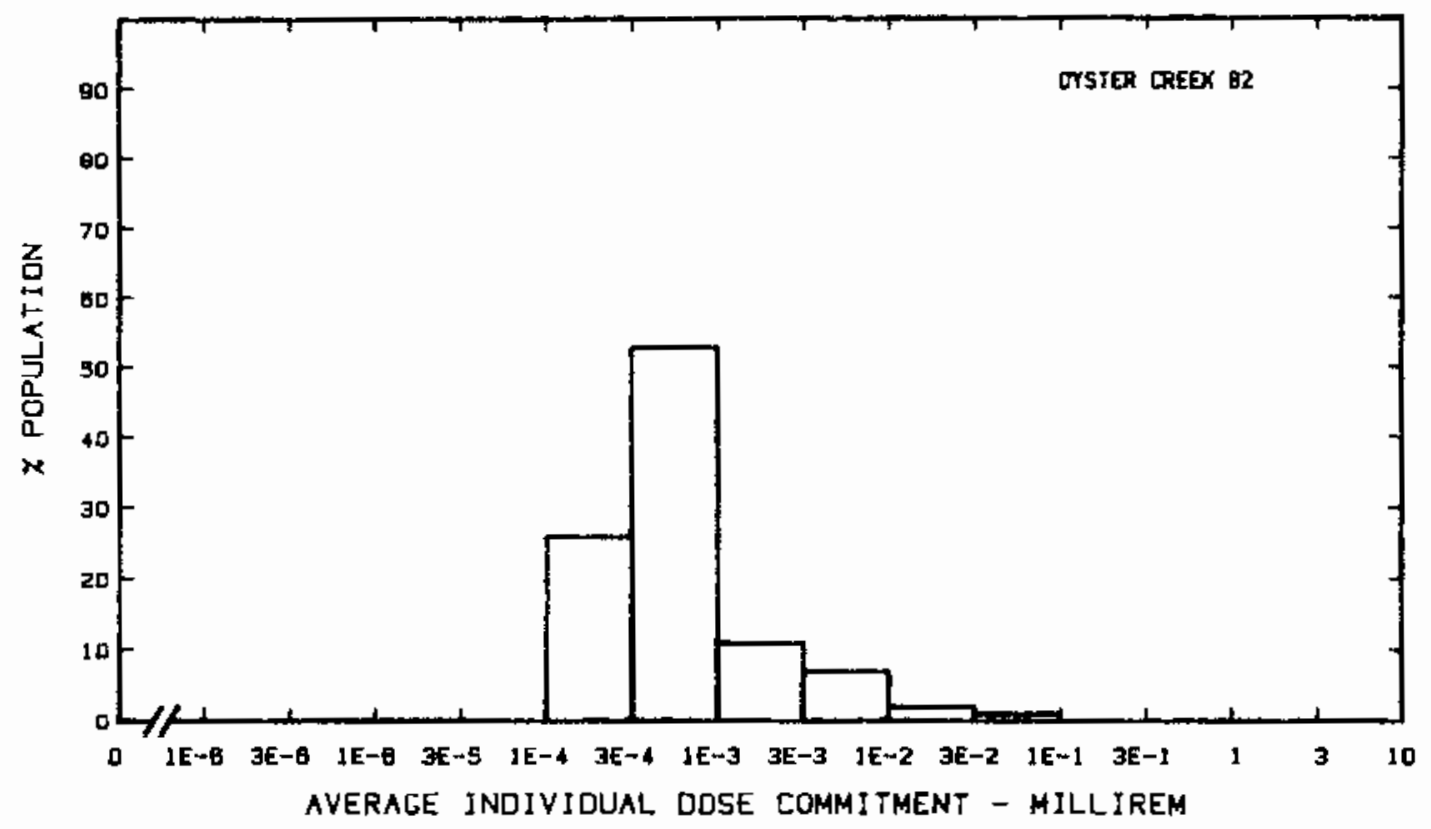


Site: PALISADES

COVERT TOWNSHIP, MICHIGAN

Location: N $42.3222^{\circ} \quad$ W $86.3153^{\circ}$

POPULATION DATA

Total Population Within 2-to-80-km Region: $1.1 \mathrm{E} 6$

Major Metropolitan Centers Within Region:

Center

Population

Location

Kalamazoo-Portage SMSA South Bend SMSA

Elkhart SMSA

Holland

Benton Harbor

280,000

280,000

140,000

26,000

15,000

$61 \mathrm{~km} \quad \mathrm{E}$

$72 \mathrm{~km} \mathrm{~S}$

$76 \mathrm{~km} \quad$ SSE

$53 \mathrm{~km} \quad \mathrm{NNE}$

$25 \mathrm{~km} \quad \mathrm{SSW}$

SITE SPECIFIC DATA - AIRBORNE PATHWAYS

Average Annual State Production

Of Crops and Animal Products

In 80-km Radius Circle

Veg: $6.8 \mathrm{E} 7$ kilogram

Milk: 2.9E8 liter

Meat: $4.5 E 7$ kilogram

Regional Productivity Factor:

Animal Grazing Factor:

0.6

0.5

Meteorology Period of Record: 1 SÉP 73 - 31 AUG 74 Recovery: 67\%

SITE SPECIFIC DATA - WATERBORNE PATHWAYS via LAKE MICHIGAN

Average Dilution Flow from
Plant: $117 \mathrm{ft}^{3} / \mathrm{s}$

Drinking Water:

Exposed Population: $51, P 09$
Dilution Factor: $3.5 \mathrm{E}-3.99$

Fish:

Edible Harvest: (b)

Dilution Factor: $0.001^{(b)}$

(a) Drinking-water dilution factor estimated by averaging dilution factor derived from FES (1972) suitably weighted for population.

(b) Since the average individual consumption rate of $20 \mathrm{~g} / \mathrm{d}$ as assumed in FES (1972) seemed unreasonably large, generic rates were used (Table A-1). 
POPULATION DOSE-COMMITMENT ESTIMATES AND

AVERAGE INDIVIDUAL DOSE-COMMITMENT HISTOGRAM FOR

\section{PAL ISADES}

Dose Commitments (person-rem) from Liquid Pathways

\begin{tabular}{|c|c|c|c|c|c|}
\hline & Total Body & GI-LII & Thyroid & Bone & Liver \\
\hline $\begin{array}{l}\text { Infant } \\
\text { Child } \\
\text { Teen } \\
\text { Adult }\end{array}$ & $\begin{array}{l}2.4 \mathrm{E}-04 \\
2.5 \mathrm{E}-02 \\
4.4 \mathrm{E}-02 \\
4.8 \mathrm{E}-01\end{array}$ & $\begin{array}{l}2.3 \mathrm{E}-04 \\
3.8 \mathrm{E}-03 \\
3.2 \mathrm{E}-03 \\
2.7 \mathrm{E}-02\end{array}$ & $\begin{array}{l}9.3 \mathrm{E}-04 \\
9.7 \mathrm{E}-03 \\
4.2 \mathrm{E}-03 \\
3.0 \mathrm{E}-02\end{array}$ & $\begin{array}{l}1.1 \mathrm{E}-04 \\
1.2 \mathrm{E}-01 \\
6.9 \mathrm{E}-02 \\
4.0 \mathrm{E}-01\end{array}$ & $\begin{array}{l}3.8 \mathrm{E}-04 \\
1.3 \mathrm{E}-01 \\
1.1 \mathrm{E}-01 \\
6.7 \mathrm{E}-01\end{array}$ \\
\hline TOTAL & $5.5 E-01$ & $3.5 \mathrm{E}-02$ & $4.5 \mathrm{E}-02$ & $5.9 \mathrm{E}-01$ & $9.1 \mathrm{E}-01$ \\
\hline
\end{tabular}

Dose Comnitments (person-rem) from Airborne Pathways Total Body GI-LII Thyroid Bone Liver Lung

$\begin{array}{lllllll}\text { Infant } & 1.0 \mathrm{E}-03 & 1.0 \mathrm{E}-03 & 1.0 \mathrm{E}-02 & 1.0 \mathrm{E}-03 & 1.0 \mathrm{E}-03 & 1.1 \mathrm{E}-03 \\ \text { Child } & 1.1 \mathrm{E}-02 & 1.1 \mathrm{E}-02 & 6.8 \mathrm{E}-02 & 1.1 \mathrm{E}-02 & 1.2 \mathrm{E}-02 & 1.3 \mathrm{E}-02 \\ \text { Teen } & 8.3 \mathrm{E}-03 & 8.2 \mathrm{E}-03 & 3.0 \mathrm{E}-02 & 8.2 \mathrm{E}-03 & 8.3 \mathrm{E}-03 & 1.0 \mathrm{E}-02 \\ \text { Adult } & 5.0 \mathrm{E}-02 & 5.0 \mathrm{E}-02 & 1.2 \mathrm{E}-01 & 4.9 \mathrm{E}-02 & 5.0 \mathrm{E}-02 & 5.5 \mathrm{E}-02 \\ \text { TOTAL } & 7.1 \mathrm{E}-02 & 7.0 \mathrm{E}-02 & 2.3 \mathrm{E}-01 & 7.0 \mathrm{E}-02 & 7.1 \mathrm{E}-02 & 7.9 \mathrm{E}-02\end{array}$

Production/Consumption factors:

Produce: $<1 \quad$ Milk: 1.2 Meat: <1

FRACTION OF POPULATION RECEIVING AN AVERAGE INDIVIDUAL TOTAL-BODY DDSE COMMITMENT FROM AIRBORNE PATHWAYS

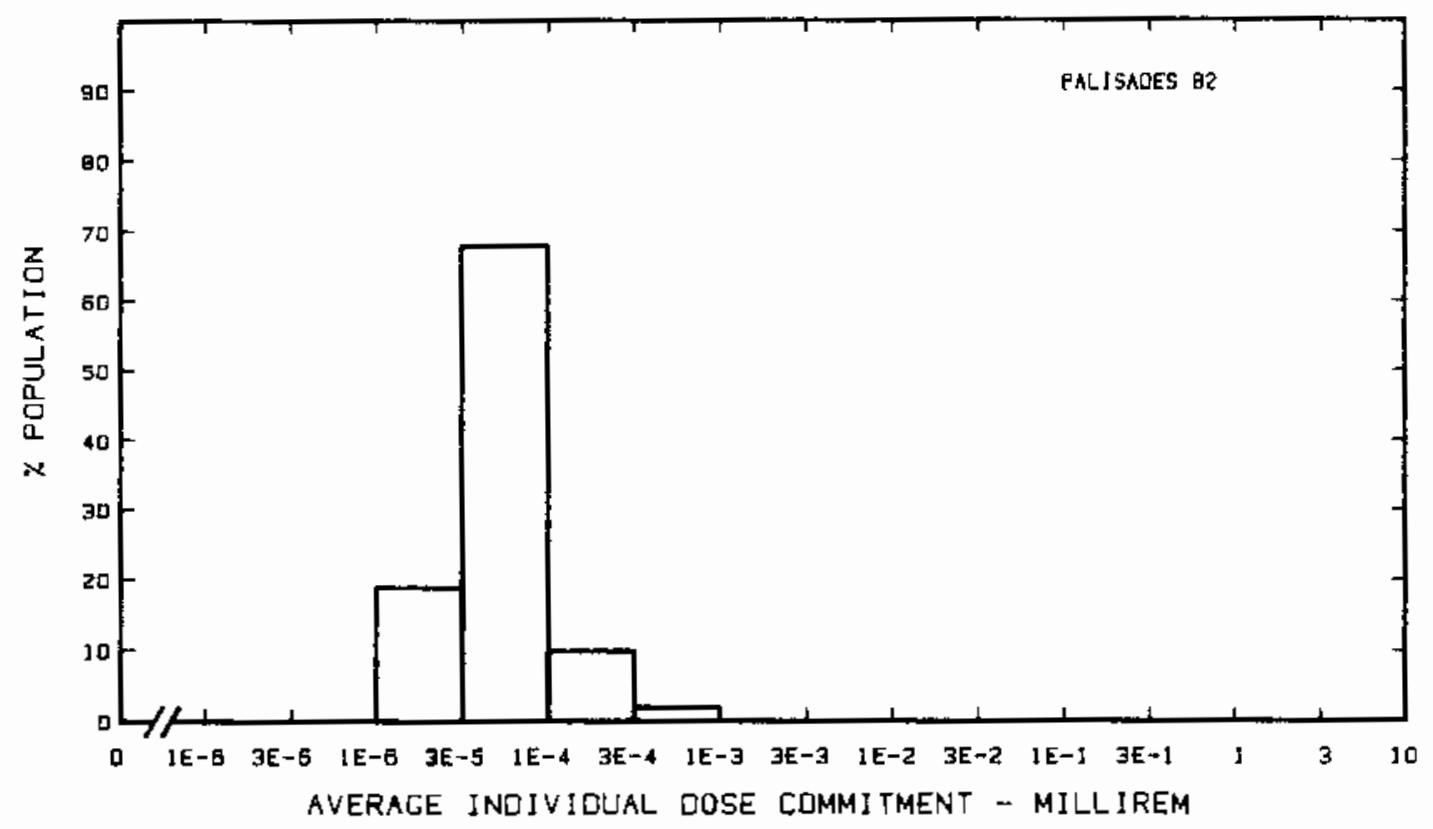


Site: PEACH BOTTOM

YORK COUNTY, PENNSYLVANIA

Location: $\quad$ N $39.7589^{\circ}$ W $76.2692^{\circ}$

POPULATION DATA

Total Population Within 2-to-80-km Region: 4.3E6

Major Metropolitan Centers Within Region:

\begin{tabular}{|c|c|c|c|}
\hline Center & Population & \multicolumn{2}{|c|}{ Location } \\
\hline Baltimore SMSA & $2,200,000$ & $60 \mathrm{~km}$ & SSh \\
\hline Harrisburg SMSA & 450,000 & $77 \mathrm{~km}$ & NNk \\
\hline WiImington SMSA & 400,000 & $62 \mathrm{~km}$ & $\mathrm{E}$ \\
\hline York SMSA & 380,000 & $45 \mathrm{~km}$ & NW \\
\hline Lancaster SMSA & 360,000 & $31 \mathrm{~km}$ & $\mathrm{~N}$ \\
\hline
\end{tabular}

SITE SPECIFIC DATA - AIRBORNE PATHWAYS

Average Annual State Production

of Crops and Animal Products

In 80-km Radius Circle

Regional Productivity Factor:

Animal Grazing Factor:
Veg: $5.3 \mathrm{E} 7 \mathrm{kilogram}$

Mi1k: 5.3E8 liter

Meat: $5.4 \mathrm{E} 7 \mathrm{ki}$ logram

0.95

0.6

Meteorology Period of Record: 1 AUG 67 - 31 JUL 71 Recovery: $72 \%$

SITE SPECIFIC DATA - WATERBORNE PATHWAYS via SUSQUEHANNA RIVER

Average River Flow

at Site: $36,000 \mathrm{ft}^{3} / \mathrm{s}$

Drinking Water:

Exposed Population: 2.2E6

Dilution Factor: 1

Fish:

Edible Harvest: (a)

0ilution Factor: $0.001^{(b)}$

(a) No fish catch data given in FES (1974), thus generic consumption rates used (Table A-1).

(b) One percent of people obtain $10 \%$ of their fish diet from river downstream from plant (FES, 1973). 


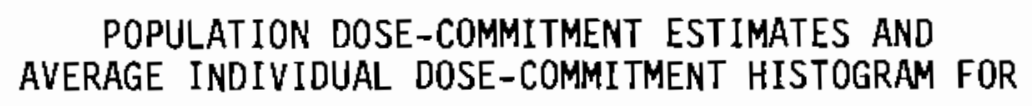

PEACH BOTTOM 2 AND 3

Dose Commitments (person-rem) from Liquid Pathways

\begin{tabular}{|c|c|c|c|c|c|}
\hline & Total Body & GI-LII & Thyroid & Bone & Liver \\
\hline $\begin{array}{l}\text { Infant } \\
\text { Child } \\
\text { Teen } \\
\text { Adult }\end{array}$ & $\begin{array}{l}\text { 2. } 7 \mathrm{E}-02 \\
4.1 \mathrm{E}-01 \\
2.3 \mathrm{E}-01 \\
2.4 \mathrm{E}-00\end{array}$ & $\begin{array}{l}\text { 2. } 5 \mathrm{E}-02 \\
1.6 \mathrm{E}-01 \\
1.3 \mathrm{E}-01 \\
1.2 \mathrm{E}-00\end{array}$ & $\begin{array}{l}3.8 \mathrm{E}-01 \\
2.7 \mathrm{E}+00 \\
7.9 \mathrm{E}-01 \\
5.5 \mathrm{E}+00\end{array}$ & $\begin{array}{l}1.0 \mathrm{E}-01 \\
1.1 \mathrm{E}+00 \\
3.1 \mathrm{E}-01 \\
2.0 \mathrm{E}+00\end{array}$ & $\begin{array}{l}1.5 \mathrm{E}-01 \\
1.4 \mathrm{E}+00 \\
5.2 \mathrm{E}-01 \\
3.4 \mathrm{E}+00\end{array}$ \\
\hline TOTAL & $3.1 \mathrm{E}+00$ & $1.6 \mathrm{E}+00$ & $9.4 E+00$ & $3.5 E+00$ & $5.4 E+00$ \\
\hline
\end{tabular}

Dose Commitments (person-rem) from Airborne Pathways

\begin{tabular}{lccccccc} 
& Total Body & GI-LII & Thyroid & Bone & Liver & Lung \\
\cline { 2 - 3 } Infant & $3.1 \mathrm{E}-02$ & $3.1 \mathrm{E}-02$ & & $1.2 \mathrm{E}-01$ & $3.1 \mathrm{E}-02$ & $3.2 \mathrm{E}-02$ & $3.3 \mathrm{E}-02$ \\
Child & $3.5 \mathrm{E}-01$ & $3.5 \mathrm{E}-01$ & $9.8 \mathrm{E}-01$ & $3.4 \mathrm{E}-01$ & $3.5 \mathrm{E}-01$ & $3.7 \mathrm{E}-01$ \\
Teen & $2.5 \mathrm{E}-01$ & $2.5 \mathrm{E}-01$ & $5.6 \mathrm{E}-01$ & $2.5 \mathrm{E}-01$ & $2.6 \mathrm{E}-01$ & $2.9 \mathrm{E}-01$ \\
Adult & $1.5 \mathrm{E}+00$ & $1.5 \mathrm{E}+00$ & $2.8 \mathrm{E}+00$ & $1.5 \mathrm{E}+00$ & $1.5 \mathrm{E}+00$ & $1.6 \mathrm{E}+00$ \\
TOTAL & $2.2 \mathrm{E}+00$ & $2.2 \mathrm{E}+00$ & $4.4 \mathrm{E}+00$ & $2.1 \mathrm{E}+00$ & $2.2 \mathrm{E}+00$ & $2.3 \mathrm{E}+00$
\end{tabular}

Production/Consumption factors:

Produce: $<1 \quad$ Milk: $<1 \quad$ Meat: <1

FRACTION OF POPULATION RECEIVING AN AVERAGE INDIVIDUAL TDTAL-BODY DOSE COMMITMENT FRDM AJRBDRNE PATHWAYS

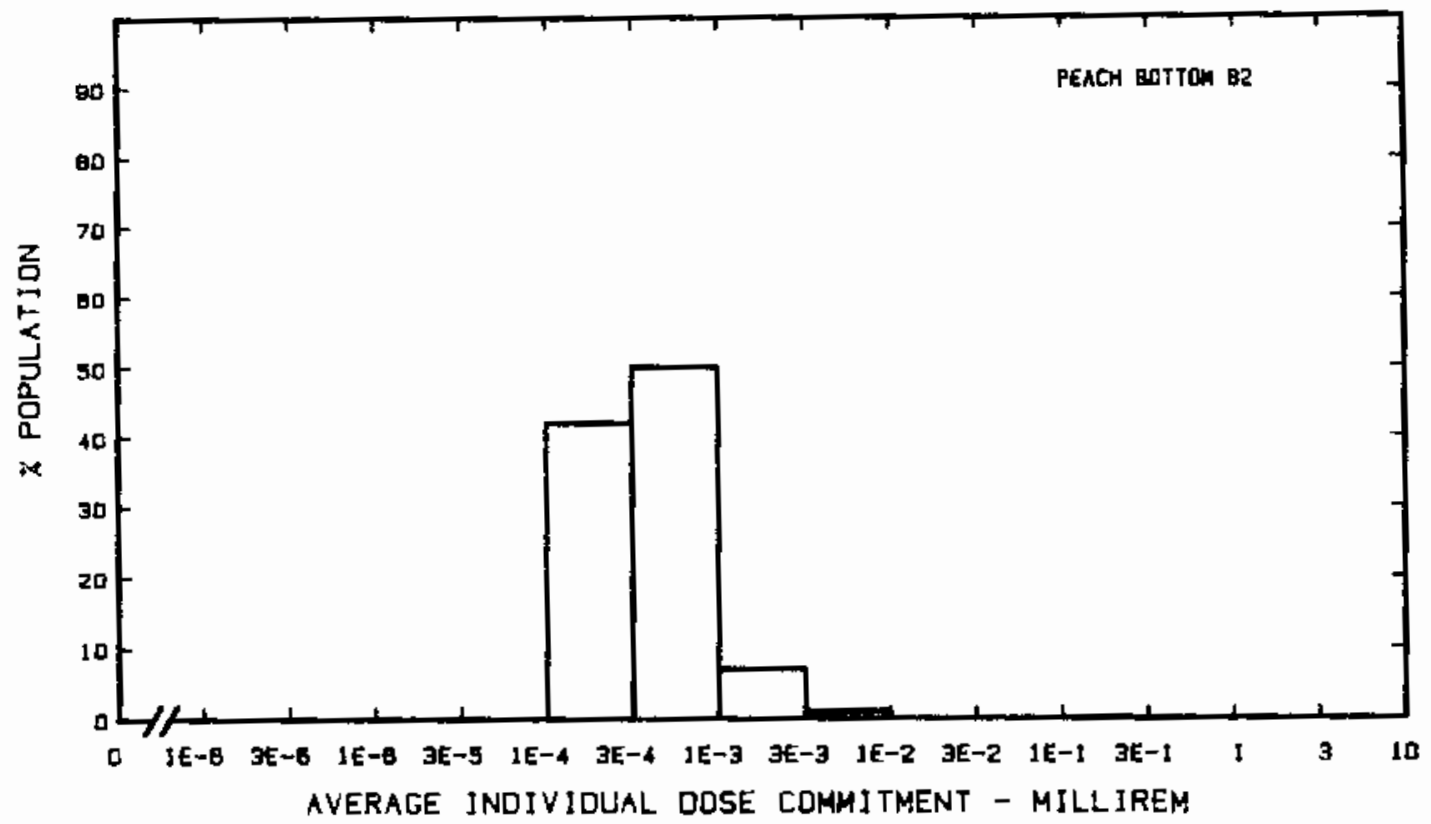


Site: PILGRIM

Location:

POPULATION DATA

Total Population Within 2-to-80-km Region: 4.3E6

Major Metropolitan Centers Within Region:

\begin{tabular}{|c|c|c|c|}
\hline Center & Population & \multicolumn{2}{|c|}{ Location } \\
\hline $\begin{array}{l}\text { Boston SMSA } \\
\text { Providence-Warwick-Pawtucket SMSA } \\
\text { New Bedford SMSA } \\
\text { Brockton SMSA } \\
\text { Fal1 River SMSA }\end{array}$ & $\begin{array}{r}2,800,000 \\
820,000 \\
170,000 \\
170,000 \\
150,000\end{array}$ & $\begin{array}{ll}61 & \mathrm{~km} \\
70 & \mathrm{~km} \\
45 & \mathrm{~km} \\
40 & \mathrm{~km} \\
55 & \mathrm{~km}\end{array}$ & $\begin{array}{l}\text { NW } \\
W \\
\text { SSW } \\
\text { WNW } \\
\text { SW }\end{array}$ \\
\hline
\end{tabular}

SITE SPECIFIC OATA - AIRBORNE PATHWAYS

Average Annual State Production

of Crops and Animal Products

In $80-\mathrm{km}$ Radius Circle

Regional Productivity Factor:

Animal Grazing Factor:

Meteorology Period of Record: 1 MAY 74 - 30 APR 75 Recovery: 93\%
Veg: $2.0 E 7$ kilogram

MiIk: 2.6E8 liter

Meat: $1.6 E 7$ kilogram

0.3

0.6

SITE SPECIFIC DATA - WATERBORNE PATHWAYS via CAPE COD BAY

Average Dilution Flow

from Plant: $16 \mathrm{ft}^{3} / \mathrm{s}$

Fish:

Edible Harvest: $2.6 \mathrm{E} 4 \mathrm{~kg} / \mathrm{yr}$

0ilution Factor: 0.001

Invertebrates:

Edible Harvest: $3.1 \mathrm{E} 4 \mathrm{~kg} / \mathrm{yr}$

Dilution Factor: 0.002 
POPULATION DOSE-COMMITMENT ESTIMATES AND

AVERAGE INDIVIOUAL OOSE-COMMITMENT HISTOGRAM FOR

PILGRIM

Oose Commitments (person-rem) from Liquid Pathways

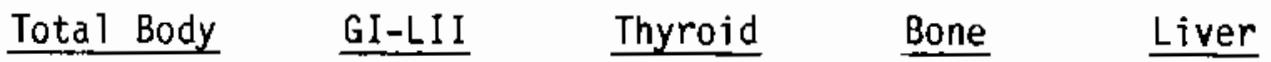

Infant

Child

Teen

Adult

$0.0 \mathrm{E}+00$

$0.0 E+00$

$5.6 \mathrm{E}-03$

4. $9 \mathrm{E}-03$

$0.0 \mathrm{E}+00$

$0.0 \mathrm{E}+00$

$0.0 \mathrm{E}+00$

3. $6 \mathrm{E}-03$

9. $8 \mathrm{E}-03$

2. $7 \mathrm{E}-06$

1. 5E-02

1. 1E-02

TOTAL

2. $2 \mathrm{E}-02$

8.5E-02

1. $9 \mathrm{E}-06$

8. $5 \mathrm{E}-03$

8.7E-03

3. 2E-02

9. $9 \mathrm{E}-02$

1. $3 E-05$

5. 1E-02

5.2E-02

Oose Commitments (person-rem) from Airborne Pathways

Total Body GI-LII Thyroid Bone Liver Lung

$\begin{array}{lllllll}\text { Infant } & 3.3 \mathrm{E}-02 & 3.3 \mathrm{E}-02 & 4.4 \mathrm{E}-02 & 3.3 \mathrm{E}-02 & 3.3 \mathrm{E}-02 & 3.4 \mathrm{E}-02 \\ \text { Child } & 3.7 \mathrm{E}-01 & 3.7 \mathrm{E}-01 & 4.4 \mathrm{E}-01 & 3.7 \mathrm{E}-01 & 3.7 \mathrm{E}-01 & 3.9 \mathrm{E}-01 \\ \text { Teen } & 2.7 \mathrm{E}-01 & 2.7 \mathrm{E}-01 & 3.0 \mathrm{E}-01 & 2.7 \mathrm{E}-01 & 2.7 \mathrm{E}-01 & 2.9 \mathrm{E}-01 \\ \text { Adult } & 1.6 \mathrm{E}+00 & 1.6 \mathrm{E}+00 & 1.8 \mathrm{E}+00 & 1.6 \mathrm{E}+00 & 1.6 \mathrm{E}+00 & 1.7 \mathrm{E}+00 \\ \text { TOTAL } & 2.3 \mathrm{E}+00 & 2.3 \mathrm{E}+00 & 2.6 \mathrm{E}+00 & 2.3 \mathrm{E}+00 & 2.3 \mathrm{E}+00 & 2.4 \mathrm{E}+00\end{array}$

Production/Consumption factors:

Produce: $<1 \quad$ Milk: <1 Meat: <1

FRACTION DF POPULATION RECEIVINC AN AVERAGE INOIVIDUAL TOTAL-BOOY DOSE CDMMITMENT FROM AIRBDRNE PATHYAYS

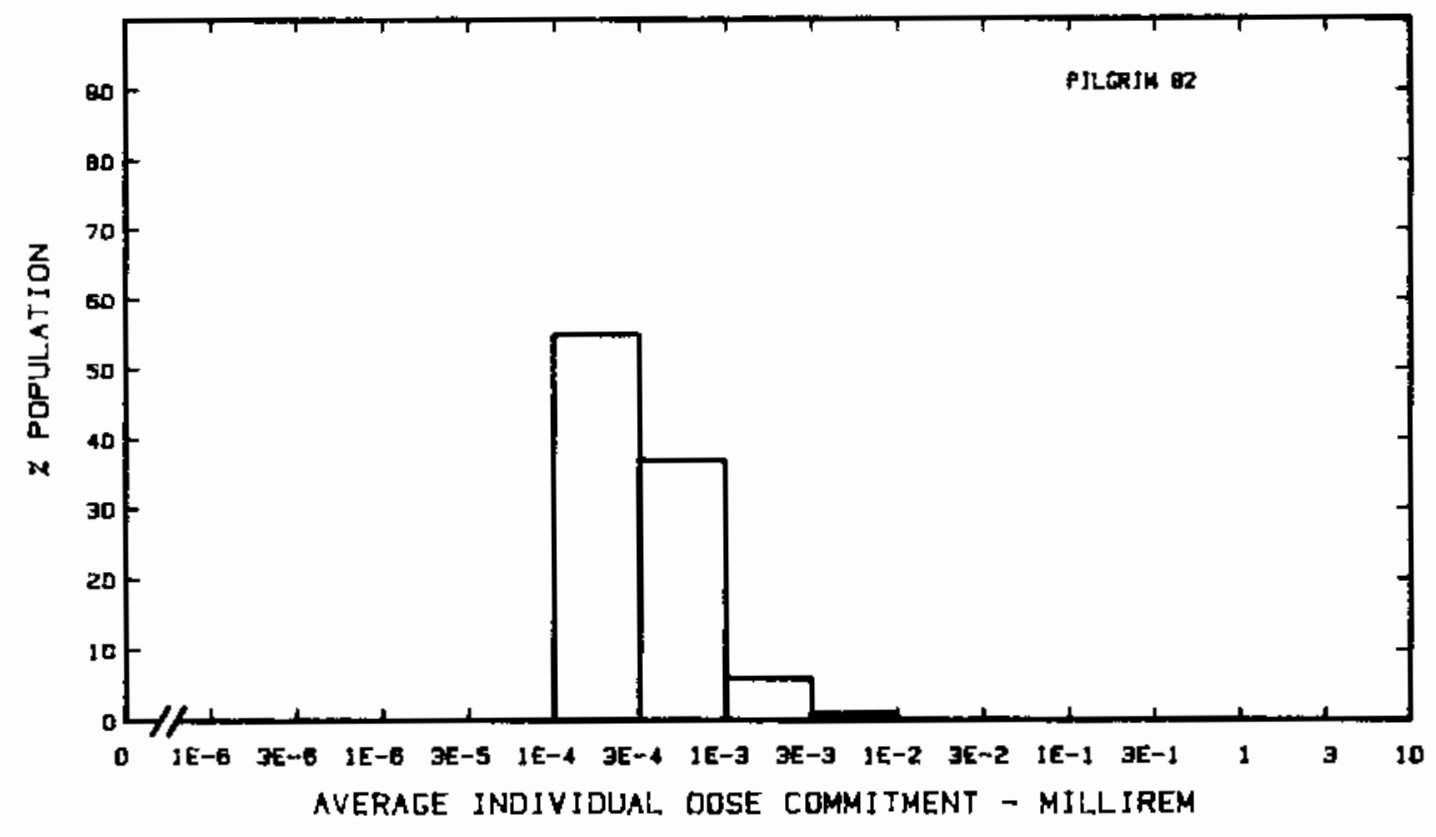


Site: POINT BEACH

MANITOWOC COUNTY, WISCONSIN

Location: $\quad$ N $44.2808^{\circ} \quad$ W $87.5361^{\circ}$

POPULATION DATA

Total Population Within 2-to-80-km Region: 6.2E5

Major Metropolitan Centers Within Region:

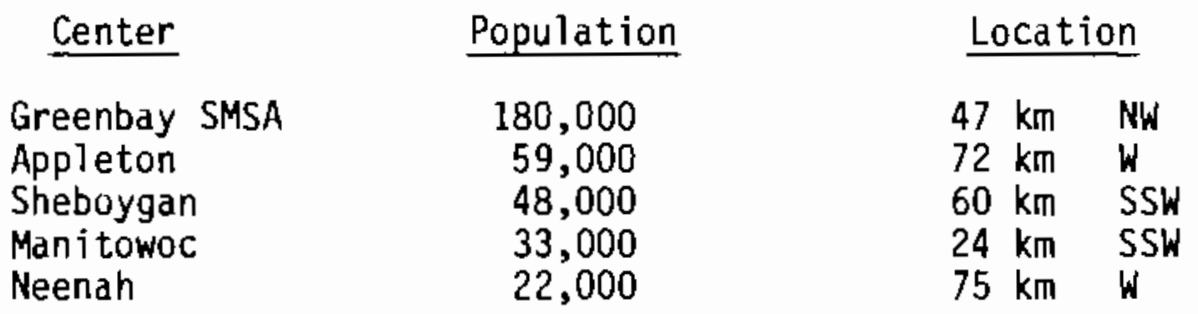

SITE SPECIFIC DATA - AIRBORNE PATHWAYS

Average Annual State Production

Veg: $7.2 \mathrm{E} 7 \mathrm{kilogram}$

of Crops and Animal Products

In $80-\mathrm{km}$ Radius Circle

Milk: 1.2Eg liter

Meat: $1.0 E 8$ kilogram

Regional Productivity Factor:

Animal Grazing Factor:

0.5

0.5

Meteorology Period of Record: 19 APR 67 - 18 APR 69 Recovery: 83\%

SITE SPECIFIC DATA - WATERBORNE PATHWAYS via LAKE MICHIGAN

Average Dilution Flow

from Plant: $770 \mathrm{ft}^{3} / \mathrm{s}$

Drinking Water:

Exposed Population: 260 (990

Fish:

Edible Harvest: $6.7 \mathrm{E4}(\mathrm{b}) \mathrm{c}(\mathrm{kg} / \mathrm{yr}$

(a) Drinking-water dilution factor estimated by averaging dilution factor derived from FES (1972), suitably weighted for population.

(b) Includes both sport and commercial fish catch (FES, 1972).

(c) Dilution factor estimated by averaging sport and commercial dilution factor derived from FES (1972). 


\section{POPULATION DOSE-COMMITMENT ESTIMATES AND \\ AVERAGE INDIVIOUAL DOSE-COMMTTMENT HISTOGRAM FOR \\ POINT BEACH 1 AND 2}

Dose Commitments (person-rem) from Liquid Pathways

Total Body GI-LII $\underline{\text { Thyroid }}$ Bone Liver

$\begin{array}{llllll}\text { Infant } & 4.9 \mathrm{E}-04 & 3.9 \mathrm{E}-04 & 2.3 \mathrm{E}-02 & 7.3 \mathrm{E}-04 & 1.3 \mathrm{E}-03 \\ \text { Child } & 1.1 \mathrm{E}-02 & 4.6 \mathrm{E}-03 & 1.7 \mathrm{E}-01 & 3.1 \mathrm{E}-02 & 4.0 \mathrm{E}-02 \\ \text { Teen } & 1.2 \mathrm{E}-02 & 2.1 \mathrm{E}-03 & 5.2 \mathrm{E}-02 & 1.6 \mathrm{E}-02 & 2.8 \mathrm{E}-02 \\ \text { Adult } & 1.3 \mathrm{E}-01 & 1.8 \mathrm{E}-02 & 3.6 \mathrm{E}-01 & 9.5 \mathrm{E}-02 & 1.7 \mathrm{E}-01 \\ \text { TOTAL } & 1.5 \mathrm{E}-01 & 2.5 \mathrm{E}-02 & 6.1 \mathrm{E}-01 & 1.4 \mathrm{E}-01 & 2.4 \mathrm{E}-01\end{array}$

Oose Commitments (person-rem) from Airborne Pathways

Total Body GI-LII Thyroid Bone Liver Lung

$\begin{array}{lllllll}\text { Infant } & 3.3 \mathrm{E}-03 & 3.3 \mathrm{E}-03 & 5.7 \mathrm{E}-03 & 4.7 \mathrm{E}-04 & 3.3 \mathrm{E}-03 & 3.3 \mathrm{E}-03 \\ \text { Child } & 5.0 \mathrm{E}-02 & 5.0 \mathrm{E}-02 & 6.7 \mathrm{E}-02 & 5.2 \mathrm{E}-03 & 5.0 \mathrm{E}-02 & 5.1 \mathrm{E}-02 \\ \text { Teen } & 3.1 \mathrm{E}-02 & 3.1 \mathrm{E}-02 & 3.7 \mathrm{E}-02 & 3.8 \mathrm{E}-03 & 3.1 \mathrm{E}-02 & 3.1 \mathrm{E}-02 \\ \text { Adult } & 1.7 \mathrm{E}-01 & 1.7 \mathrm{E}-01 & 1.9 \mathrm{E}-01 & 2.3 \mathrm{E}-02 & 1.7 \mathrm{E}-01 & 1.7 \mathrm{E}-01 \\ \text { T0TAL } & 2.6 \mathrm{E}-01 & 2.6 \mathrm{E}-01 & 3.0 \mathrm{E}-01 & 3.2 \mathrm{E}-02 & 2.6 \mathrm{E}-01 & 2.6 \mathrm{E}-01\end{array}$

Production/Consumption factors:

Produce: <1 Milk: $7.3 \quad$ Meat: 1.0

FRACTION DF POPULATION RECEIVING AN AVERAGE INDIVIDUAL TOTAL-BODY DDSE COMMITMENT FROM AIRBDRNE PATHWAYS

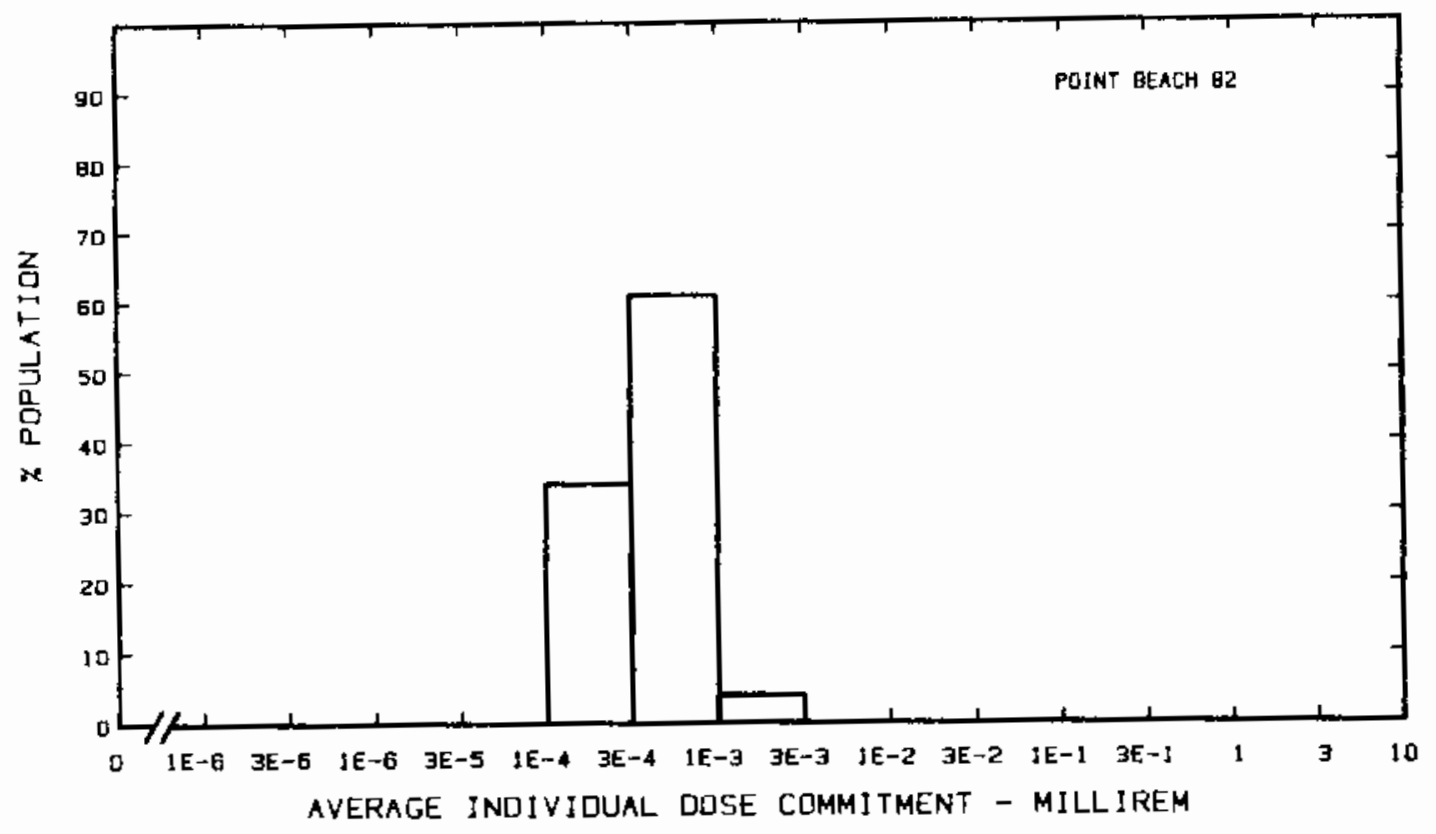


Site: PRAIRIE ISLANO

RED WING, MINNESOTA

Location: N $44.6219^{\circ}$ W $92.6331^{\circ}$

POPULATION OATA

Total Population Within 2-to-80-km Region: 2.2E6

Major Metropotitan Centers Within Region:

Center Population Location

Minneapolis-St. Paul SMSA 2,100,000 $63 \mathrm{~km}$ NW

Rochester SMSA $\quad 92,000 \quad 68 \mathrm{~km} \quad$ SSE

Owatonna

Faribault

19,000

Redwing

16,000

$77 \mathrm{~km} \quad \mathrm{SW}$

$63 \mathrm{~km} \mathrm{SW}$

$10 \mathrm{~km} \quad \mathrm{SE}$

SITE SPECIFIC DATA - AIRBORNE PATHWAYS

Average Annual State Production

Veg: $1.2 E 8$ kilogram

of Crops and Animal Products

In 80-km Radius Circle

Milk: 4.0E8 liter

Meat: 1.0E8 kilogram

Regional Productivity Factor:

Animal Grazing Factor:

1

0.5

Meteorology Period of Record: 22 MAR 74 - 21 MAR 75 Recovery: 65\%

SITE SPECIFIC DATA - WATERBORNE PATHWAYS via MISSISSIPPI RIVER

Average River Flow

at Site: $15,000 \mathrm{ft}^{3} / \mathrm{s}$

Orinking Water:

Exposed Population: None

Fish:

Edible Harvest: $6.8 \mathrm{E} 5 \mathrm{~kg} / \mathrm{yr}$

0ilution Factor: 1 
POPULATION DOSE-COMMITMENT ESTIMATES AND

AVERAGE INDIVIDUAL DOSE-COMMITMENT HISTOGRAM FOR

PRAIRIE ISLAND 1 AND 2

Dose Commitments (person-rem) from Liquid Pathways

\begin{tabular}{|c|c|c|c|c|c|}
\hline & Total Body & $G I-L I I$ & Thyroid & Bone & Liver \\
\hline $\begin{array}{l}\text { Infant } \\
\text { Child } \\
\text { Teen } \\
\text { Adult }\end{array}$ & $\begin{array}{l}0.0 E+00 \\
3.3 E-04 \\
3.0 E-04 \\
2.4 E-03\end{array}$ & $\begin{array}{l}0.0 E+00 \\
3.3 E-04 \\
3.0 E-04 \\
2.4 E-03\end{array}$ & $\begin{array}{l}0.0 \mathrm{E}+00 \\
3.3 \mathrm{E}-04 \\
3.0 \mathrm{E}-04 \\
2.4 \mathrm{E}-03\end{array}$ & $\begin{array}{l}0.0 \mathrm{E}+00 \\
2.5 \mathrm{E}-09 \\
1.4 \mathrm{E}-09 \\
8.3 \mathrm{E}-09\end{array}$ & $\begin{array}{l}0.0 \mathrm{E}+00 \\
3.3 \mathrm{E}-04 \\
3.0 \mathrm{E}-04 \\
2.4 \mathrm{E}-03\end{array}$ \\
\hline TOTAL & $3.0 \mathrm{E}-03$ & 3. $1 \mathrm{E}-03$ & 3. $0 \mathrm{E}-03$ & $1.2 \mathrm{E}-08$ & $3.0 \mathrm{E}-03$ \\
\hline
\end{tabular}

Dose Commitments (person-rem) from Airborne Pathways

Total Body GI-LII Thyroid Bone Liver Lung

$\begin{array}{lllllll}\text { Infant } & 8.4 \mathrm{E}-04 & 8.3 \mathrm{E}-04 & 6.2 \mathrm{E}-03 & 2.6 \mathrm{E}-04 & 8.5 \mathrm{E}-04 & 8.5 \mathrm{E}-04 \\ \text { Child } & 1.2 \mathrm{E}-02 & 1.2 \mathrm{E}-02 & 4.7 \mathrm{E}-02 & 2.8 \mathrm{E}-03 & 1.2 \mathrm{E}-02 & 1.2 \mathrm{E}-02 \\ \text { Teen } & 7.8 \mathrm{E}-03 & 7.8 \mathrm{E}-03 & 2.1 \mathrm{E}-02 & 2.0 \mathrm{E}-03 & 7.9 \mathrm{E}-03 & 8.2 \mathrm{E}-03 \\ \text { Adult } & 4.4 \mathrm{E}-02 & 4.4 \mathrm{E}-02 & 8.8 \mathrm{E}-02 & 1.2 \mathrm{E}-02 & 4.4 \mathrm{E}-02 & 4.5 \mathrm{E}-\mathrm{D2} \\ \text { TOTAL } & 6.4 \mathrm{E}-02 & 6.4 \mathrm{E}-02 & 1.6 \mathrm{E}-01 & 1.7 \mathrm{E}-02 & 6.5 \mathrm{E}-02 & 6.6 \mathrm{E}-02\end{array}$

Production/Consumption factors:

Produce: $<1 \quad$ Milk: $1.4 \quad$ Meat: <1

FRACTION DF POPULATION RECEIVING AN AVERAGE INDIVIOUAL

TOTAL-BODY DOSE COMMITMENT FROM AIRBORNE PATHWAYS

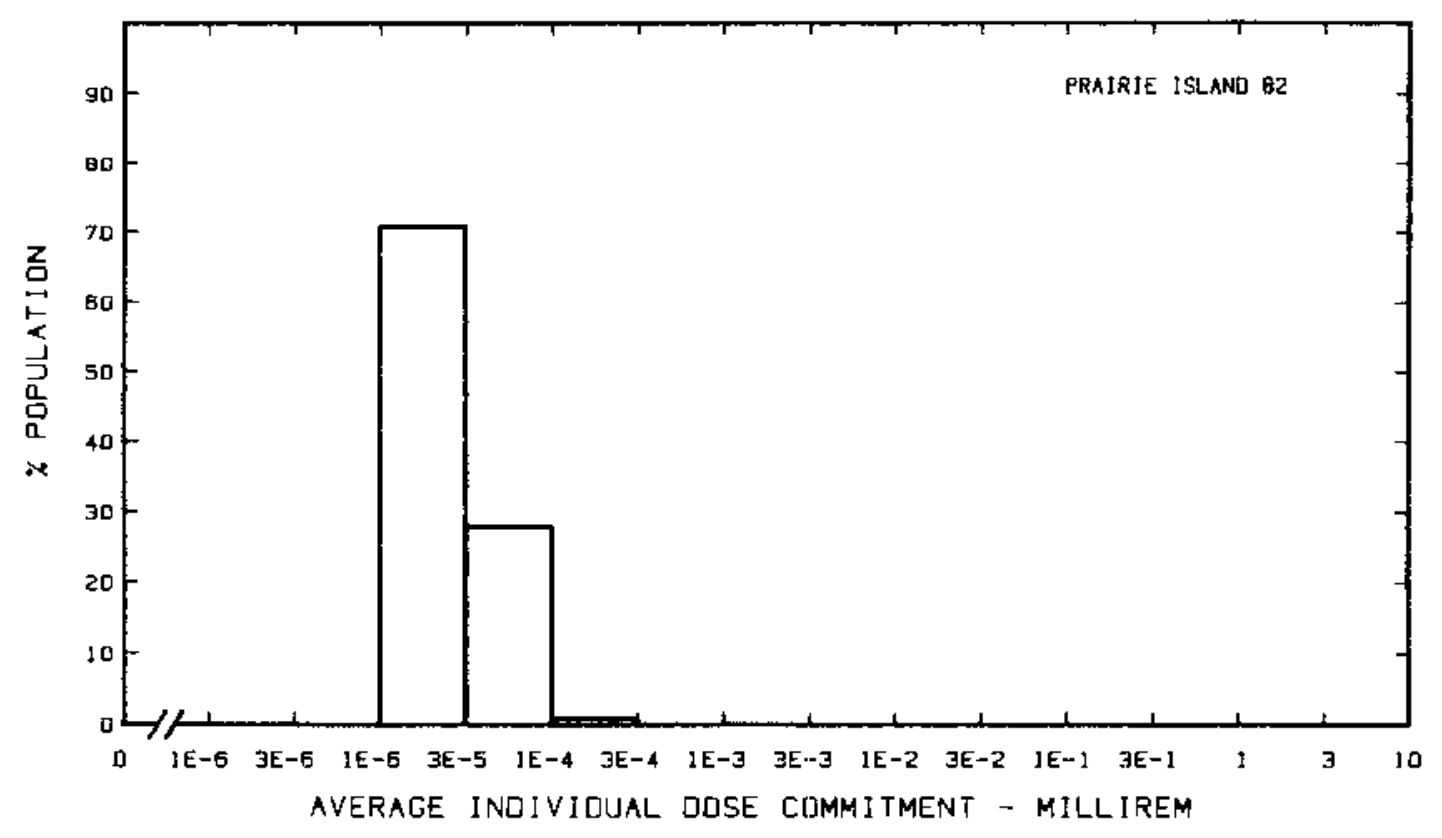


Site: QUAD CITIES

ROCK ISLAND, ILLINOIS

Location: $\quad \mathrm{N} 41.7261^{\circ} \quad$ W $90.3100^{\circ}$

POPULATION DATA

Total Population Within 2-to-80-km Region: 7.2E5

Major Metropolitan Centers Within Region:

Center $\quad$ Population Location

Davenport-Rock Island-Moline SMSA $380,000 \quad 30 \mathrm{~km} \quad \mathrm{SW}$

Muscatine

Sterling

Dixon

23,000

16,000

$70 \mathrm{~km} \quad \mathrm{WSW}$

Kewanee

16,000

$52 \mathrm{~km} \quad \mathrm{E}$

$70 \mathrm{~km} \quad \mathrm{E}$

$62 \mathrm{~km} \quad \mathrm{SSE}$

SITE SPECIFIC DATA - AIRBORNE PATHWAYS

Average Annual State Production

Veg: 1.1E8 kilogram

of Crops and Animal Products

In 80-km Radius Circle

Mi1k: 1.8 E8 liter

Meat: 1.9E8 kilogram

Regional Productivity Factor:

Animal Grazing Factor:

1

0.5

Meteorology Period of Record: 1 JAN 74 - 31 DEC 75 Recovery: 88\%

SITE SPECIFIC DATA - WATERBORNE PATHWAYS via MISSISSIPPI RIVER

Average River Flow

at Site: $46,000 \mathrm{ft}^{3} / \mathrm{s}$

Drinking Water:

Exposed Population: 380,000 (a)

Dilution Factor: 1

Fish:

Edible Harvest: $2.1 \mathrm{E}\left(_{\mathrm{b})}^{(\mathrm{b})} \mathrm{kg} / \mathrm{yr}\right.$
Dilution Factor: $0.5^{\mathrm{b}}$

(a) All people in Davenport SMSA assumed to drink river water.

(b) Assumes $1 / 2$ fish harvest caught below plant. 
POPULATION DOSE-COMMITMENT ESTIMATES AND
AVERAGE INDIVIDUAL DOSE-COMMITMENT HISTOGRAM FOR

QUAD CITIES 1 AND 2

Dose Commitments (person-rem) from Liquid Pathways

Total Body GI-LII Thyroid Bone Liver

$\begin{array}{llllll}\text { Infant } & 2.2 \mathrm{E}-04 & 1.5 \mathrm{E}-04 & 4.2 \mathrm{E}-03 & 9.2 \mathrm{E}-04 & 8.1 \mathrm{E}-04 \\ \text { Child } & 1.1 \mathrm{E}-02 & 5.4 \mathrm{E}-02 & 3.1 \mathrm{E}-02 & 5.9 \mathrm{E}-02 & 5.5 \mathrm{E}-02 \\ \text { Teen } & 1.6 \mathrm{E}-02 & 1.1 \mathrm{E}-01 & 9.5 \mathrm{E}-03 & 3.2 \mathrm{E}-02 & 4.2 \mathrm{E}-02 \\ \text { Adult } & 1.7 \mathrm{E}-01 & 9.5 \mathrm{E}-01 & 6.6 \mathrm{E}-02 & 1.9 \mathrm{E}-01 & 2.5 \mathrm{E}-\mathrm{Dl} \\ \text { TOTAL } & 2.0 \mathrm{E}-01 & 1.1 \mathrm{E}+00 & 1.1 \mathrm{E}-01 & 2.8 \mathrm{E}-01 & 3.5 \mathrm{E}-01\end{array}$

Dose Commitments (person-rem) from Airborne Pathways

\begin{tabular}{lccccccc} 
& Total Body & GI-LII & Thyroid & Bone & Liver & Lung \\
\cline { 2 - 3 } Infant & $1.2 \mathrm{E}-02$ & $1.2 \mathrm{E}-02$ & $1.2 \mathrm{E}-01$ & $1.2 \mathrm{E}-02$ & $1.3 \mathrm{E}-02$ & $1.2 \mathrm{E}-02$ \\
Child & $1.4 \mathrm{E}-01$ & $1.4 \mathrm{E}-01$ & $1.2 \mathrm{E}+00$ & $1.7 \mathrm{E}-01$ & $1.4 \mathrm{E}-01$ & $1.4 \mathrm{E}-01$ \\
Teen & $9.9 \mathrm{E}-02$ & $1.0 \mathrm{E}-01$ & $5.7 \mathrm{E}-01$ & $1.1 \mathrm{E}-01$ & $1.0 \mathrm{E}-01$ & $1.1 \mathrm{E}-01$ \\
Adult & $5.9 \mathrm{E}-01$ & $6.0 \mathrm{E}-01$ & $2.4 \mathrm{E}+00$ & $6.1 \mathrm{E}-01$ & $6.0 \mathrm{E}-01$ & $6.2 \mathrm{E}-01$ \\
TOTAL & $8.4 \mathrm{E}-01$ & $8.5 \mathrm{E}-01$ & $4.3 \mathrm{E}+00$ & $8.9 \mathrm{E}-01$ & $8.6 \mathrm{E}-01$ & $8.8 \mathrm{E}-01$
\end{tabular}

Production/Consumption factors:

Produce: $<1$

Milk: 1.9

Meat: 3.3

FRACTIDN OF POPULATION RECEIVING AN AVERAGE INDIVIOUAL TOTAL $-B O D Y$ DDSE CDMYITMENT FRDM AIRBORNE PATHWAYS

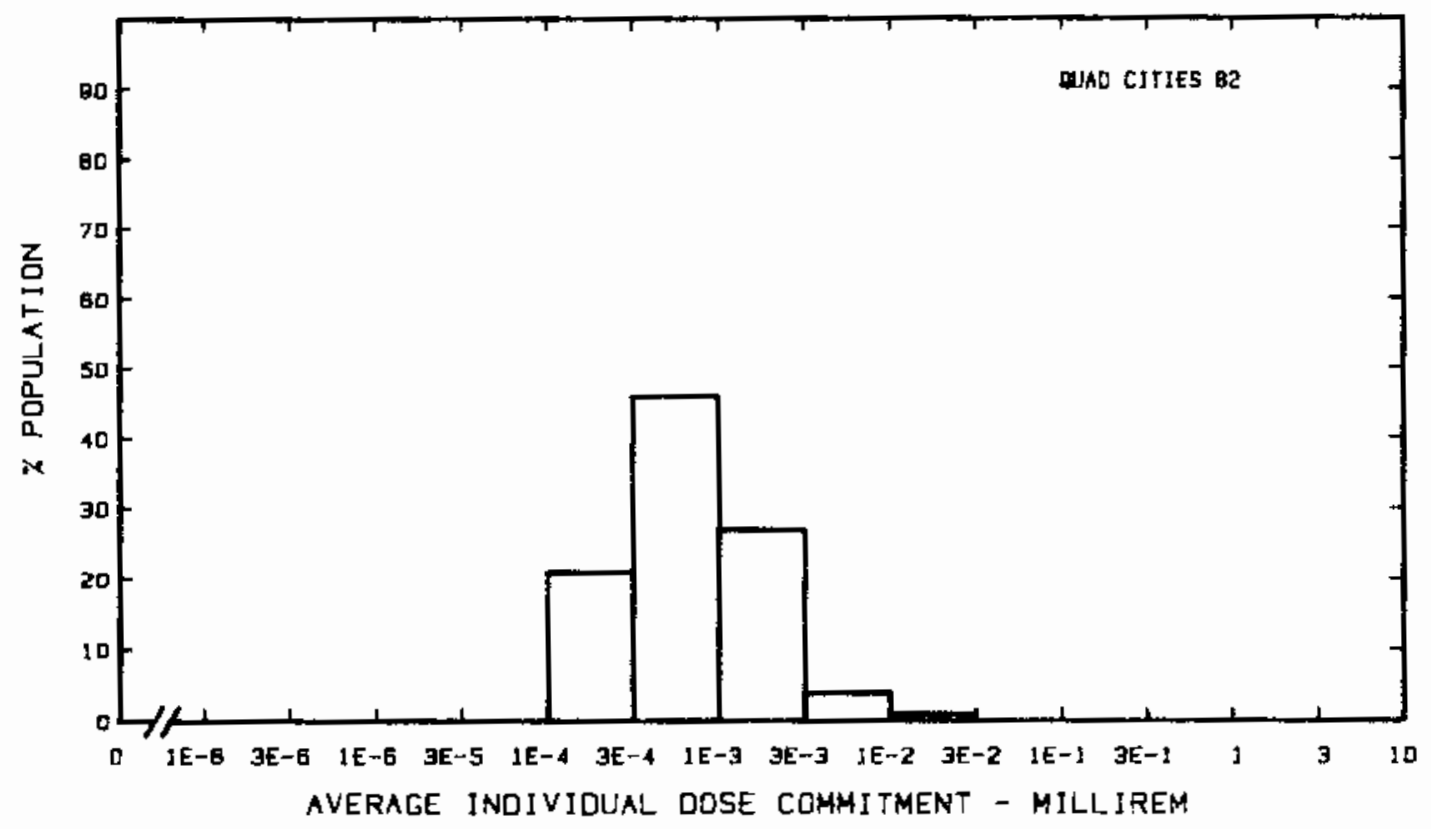


Site: RANCHO SECO

SACRAMENTO COUNTY, CALIFORNIA

Location: N $38.3444^{\circ}$ W $121.1200^{\circ}$

POPULATION DATA

Total Population Within 2-to-80-km Region: 1.8E6

Major Metropolitan Centers Within Region:

$\quad$ Center
Sacramento SMSA
Stockton SMSA
Modesto SMSA
Ant ioch
Davis

Population

Location

Sacramento SMSA

Stockton SMSA

$1,000, \mathrm{D} 00$

350,000

210,000

43,000

Davis

37,000

$\begin{array}{lll}42 & \mathrm{~km} & \text { NW } \\ 45 & \mathrm{~km} & \text { SSW } \\ 79 & \mathrm{~km} & \mathrm{~S} \\ 71 & \mathrm{~km} & \text { WSW } \\ 58 & \mathrm{~km} & \text { WNW }\end{array}$

SITE SPECIFIC DATA - AIRBORNE PATHWAYS

Average Annual State Production

of Crops and Animal Products

In $80-\mathrm{km}$ Radius Circle

Regional Productivity Factor:

Animal Grazing Factor:

Meteorology Period of Record: 1 FEB 75 - 31 JAN 76 Recovery: 98\%
Veg: $4.8 \mathrm{E} 7 \mathrm{kilogram}$

Milk: 2.3E8 liter

Meat: $5.0 E 7$ kilogram

1

0.9

SITE SPECIFIC DATA - WATERBORNE PATHWAYS via COSUMNES \& MOKELUMNE RIVERS

Average Dilution Flow

from Plant: $7.0 \mathrm{ft}^{3} / \mathrm{s}$

Drinking Water:

Exposed Population: None

Fish:

Edible Haryest: (a)

Dilution Factor: 0.005

(a) One percent of population is assumed to obtain fish from river. Average individual consumption rate given in FES (1973) used in liew of catch data. 


\section{POPULATION DOSE-COMMITMENT ESTIMATES ANO \\ AVERAGE INDIVIDUAL OOSE-COMMITMENT HISTOGRAM FOR \\ RANCHO SECO}

Dose Commitments (person-rem) from Liquid Pathways

\begin{tabular}{|c|c|c|c|c|c|}
\hline & Total Body & GI-LII & Thyroid & Bone & Liver \\
\hline $\begin{array}{l}\text { Infant } \\
\text { Child } \\
\text { Teen } \\
\text { Adult }\end{array}$ & $\begin{array}{l}0.0 \mathrm{E}+00 \\
2.0 \mathrm{E}-02 \\
3.9 \mathrm{E}-02 \\
4.2 \mathrm{E}-01\end{array}$ & $\begin{array}{l}0.0 \mathrm{E}+00 \\
4.4 \mathrm{E}-03 \\
9.0 \mathrm{E}-03 \\
7.8 \mathrm{E}-02\end{array}$ & $\begin{array}{l}0.0 \mathrm{E}+00 \\
4.6 \mathrm{E}-03 \\
3.3 \mathrm{E}-03 \\
2.2 \mathrm{E}-02\end{array}$ & $\begin{array}{l}0.0 \mathrm{E}+00 \\
1.0 \mathrm{E}-01 \\
6.1 \mathrm{E}-02 \\
3.5 \mathrm{E}-01\end{array}$ & $\begin{array}{l}0.0 \mathrm{E}+00 \\
1.2 \mathrm{E}-01 \\
9.8 \mathrm{E}-02 \\
5.8 \mathrm{E}-01\end{array}$ \\
\hline OTAL & $4.8 \mathrm{E}-01$ & $9.1 \mathrm{E}-02$ & $3.0 E-02$ & $5.1 \mathrm{E}-01$ & 8. $0 E-01$ \\
\hline
\end{tabular}

Oose Commitments (person-rem) from Airborne Pathways

Total Body GI-LII Thyroid Bone Liver

$\begin{array}{lllllll}\text { Infant } & 1.2 \mathrm{E}-03 & 1.2 \mathrm{E}-03 & 1.7 \mathrm{E}-03 & 8.1 \mathrm{E}-04 & 1.2 \mathrm{E}-03 & 1.4 \mathrm{E}-03 \\ \text { Child } & 1.5 \mathrm{E}-02 & 1.5 \mathrm{E}-02 & 1.7 \mathrm{E}-02 & 9.0 \mathrm{E}-03 & 1.5 \mathrm{E}-02 & 1.7 \mathrm{E}-02 \\ \text { Teen } & 1.0 \mathrm{E}-02 & 1.0 \mathrm{E}-02 & 1.1 \mathrm{E}-02 & 6.5 \mathrm{E}-03 & 1.0 \mathrm{E}-02 & 1.3 \mathrm{E}-02 \\ \text { Adult } & 5.8 \mathrm{E}-02 & 6.0 \mathrm{E}-02 & 6.1 \mathrm{E}-02 & 4.0 \mathrm{E}-02 & 5.8 \mathrm{E}-02 & 6.9 \mathrm{E}-02 \\ \text { TOTAL } & 8.4 \mathrm{E}-02 & 8.7 \mathrm{E}-02 & 9.1 \mathrm{E}-02 & 5.6 \mathrm{E}-02 & 8.4 \mathrm{E}-02 & 1.0 \mathrm{E}-01\end{array}$

Production/Consumption factors:

Produce: < Milk: 1.0 Meat: <1

FRACTION OF POPULATION RECEIVING AN AVERAGE INUIVICUAL TOTAL-EOCY DOSE COMMITMENT FROM AIRBORNE PATHWAYS

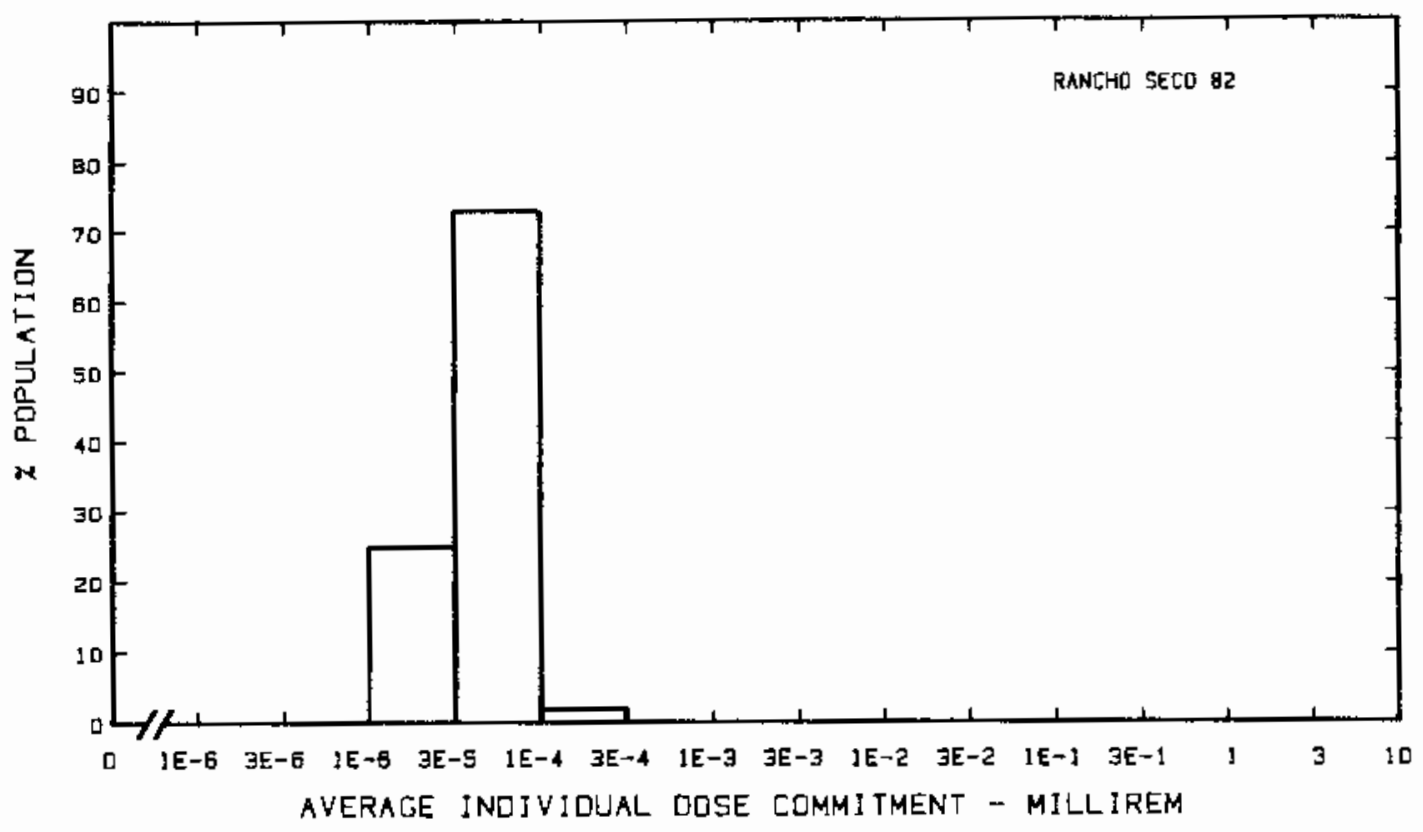


Site: H. B. ROBINSON

HARTSVILLE, SOUTH CAROLINA

Location: $\quad$ N $34.4858^{\circ} \quad$ W $80.1586^{\circ}$

POPULATION DATA

Total Population Within 2-to-80-km Region: 6.7E5

Major Metropolitan Centers Within Region:

Center Population Location

Florence $\quad 30,000 \quad 42 \mathrm{~km} \quad$ ESE

Sumter $\quad 25,000 \quad 56 \mathrm{~km} \quad$ SSW

Monroe $\quad 13,0 \mathrm{DO} \quad 74 \mathrm{~km} \quad$ NNW

Lancaster $\quad 10,000 \quad 66 \mathrm{~km} \quad \mathrm{WNW}$

SITE SPECIFIC DATA - AIRBORNE PATHWAYS

Average Annual State Production

of Crops and Animal Products

In $80-\mathrm{km}$ Radius Circle

Regional Productivity Factor:

Animal Grazing Factor:

Meteorology Period of Record: 1 JAN 75 - 31 DEC 75 Recovery: 94\%
Veg: $7.4 \mathrm{E} 6$ kilogram

Mi lk: 5.7E7 liter

Meat: 5.0E7 kilogran

1

0.8

SITE SPECIFIC DATA - WATERBORNE PATHWAYS via LAKE ROBINSON

Average Dilution Flow

from Plant: $690 \mathrm{ft}^{3} / \mathrm{s}$

Drinking Water:

Exposed Population: None

Fish:

Edible Harvest: $1.8^{(\mathrm{a})}(\mathrm{bg} / \mathrm{yr}$

(a) Average individual consumption rate as given in the FES (1975) used in lieu of catch data.

(b) Ten percent of population consumes fish taken from water diluted by a factor of 0.2 (FES, 1975). 
POPULATION DOSE-COMAITMENT ESTIMATES AND AVERAGE INDIVIDUAL DOSE-COMAI TMENT HISTOGRAM FOR

H. B. ROBINSON 2

Dose Commitments (person-rem) from Liquid Pathways

Total Body

$\underline{\text { GI-LII }}$

Thyroid

Bone

Liver

Infant

Child

Teen

Adult

$$
\text { D. } D E+00
$$

$0.0 \mathrm{E}+00$

2. $2 \mathrm{E}-03$

4.7E-04

$0.0 \mathrm{E}+00$

$0.0 \mathrm{E}+00$

7.5E-04

1. $3 \mathrm{E}-02$

3. $6 \mathrm{E}-03$

9. $3 \mathrm{E}-04$

5.5E-04

8. DE-03

8.0E-D3

3. $7 \mathrm{E}-03$

4. $8 \mathrm{E}-02$

$0.0 \mathrm{E}+0 \mathrm{D}$

TOTAL

4. 3E-02

9. $4 \mathrm{E}-03$

5. $0 \mathrm{E}-03$

6. $9 \mathrm{E}-02$

1.0E-02

8.7E-03

5. IE-02

7. $0 \mathrm{E}-02$

Dose Commitments (person-rem) from Airborne Pathways Total Body GI-LII Thyroid Bone Liver Lung

Infant

Child

$5.3 \mathrm{E}-05$

$5.3 \mathrm{E}-05$

2.3E-04

$5.0 \mathrm{E}-05$

5. $3 \mathrm{E}-05$

$5.7 \mathrm{E}-05$

Teen

6. $0 \mathrm{E}-04$

6. $0 \mathrm{E}-04$

$1.6 \mathrm{E}-03$

$5.5 \mathrm{E}-04$

4. $4 \mathrm{E}-04$

8. $3 \mathrm{E}-04$

4. $0 \mathrm{E}-04$

$6.0 \mathrm{E}-04$

$6.6 \mathrm{E}-04$

Adult

2. $6 \mathrm{E}-03$

2.7 -03

4. $0 \mathrm{E}-03$

2. $4 \mathrm{E}-03$

4. $4 \mathrm{E}-04$

5. $1 \mathrm{E}-04$

TOTAL

3. $7 \mathrm{E}-03$

3. $7 \mathrm{E}-03$

6.7E-03 3.4E-03

2. $6 \mathrm{E}-03$

2. $9 \mathrm{E}-03$

$3.7 \mathrm{E}-03$

4. $1 \mathrm{E}-03$

Production/Consumption factors:

Produce: $<1 \quad$ Milk: $<1 \quad$ Meat: $<1$

FRACTIDN DF PDPULATION RECEIVING AN AVERAGE INDJVIDUAL TOTAL-BODY ODSE COMMITMENT FRDM AIRBORNE PATHWAYS

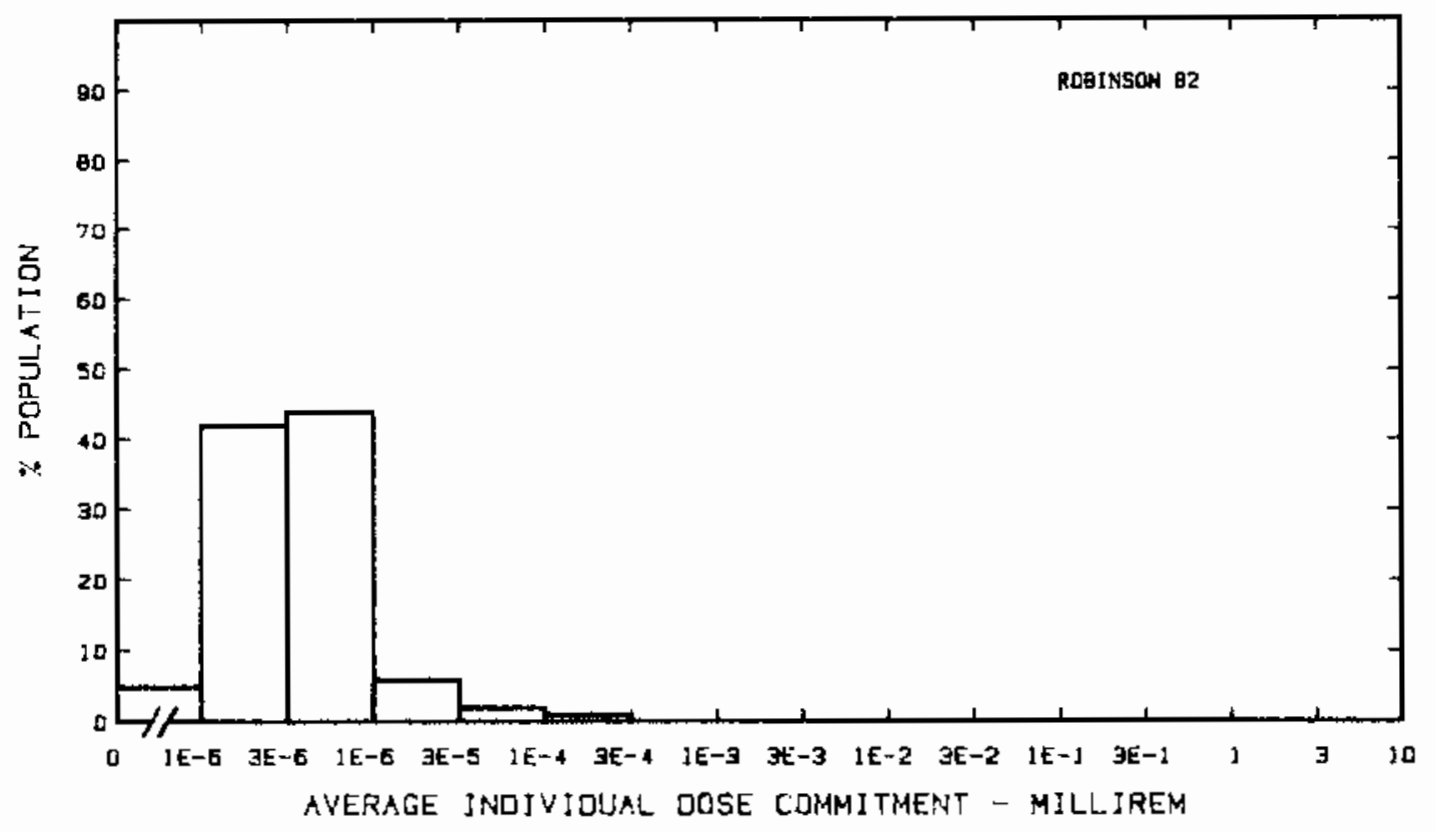


Site: ST. LUCIE

FORT PIERCE, FLORIDA

Location: $\quad$ N $27.3486^{\circ} \quad$ W $80.2464^{1}$

POPULATION DATA

Total Population Within 2-to-80-km Region: 5.8 E5

Major Metropolitan Centers Within Region:

Center

West Palm Beach

Ft. Pierce

Riviera Beach

Vero Beach

$\mathrm{Pa}$ Im Beach
Population

63,000

34,000

26,000

16,000

10,000
Location

$73 \mathrm{~km} \quad$ SSE

$14 \mathrm{~km} \quad \mathrm{NW}$

$65 \mathrm{~km} \quad \mathrm{SSE}$

$36 \mathrm{~km} \quad \mathrm{NNW}$

$72 \mathrm{~km} \quad \mathrm{SSE}$

SITE SPECIFIC DATA - AIRBORNE PATHWAYS

Average Annual State Production

of Crops and Animal Products

In $80-\mathrm{km}$ Radius Circle

Regional Productivity Factor:

Animal Grazing Factor:

Meteorology Period of Record: 1 JAN 76 - 31 DEC 76 Recovery: 92\%
Milk: $1.1 E 8$ liter

Meat: $7.2 \mathrm{E} 7$ kilogram

0.5

1
Veg: $2.8 \mathrm{E} 7 \mathrm{kilogram}$

SITE SPECIFIC DATA - WATERBORNE PATHWAYS via ATLANTIC OCEAN

Average Dilution Flow

from Plant: $1,100 \mathrm{ft}^{3} / \mathrm{s}$

Fish:

Edible Harvest: $2.6 E 5 \mathrm{~kg} / \mathrm{yr}$

Invertebrates:

Edible Harvest: $2.7 E 4 \mathrm{~kg} / \mathrm{yr}$
Dilution Factor: $0.005 \mathrm{~g}$

(a) Dilution factors as given in FES (1973). 
POPULATION DOSE-COMMITMENT ESTIMATES AND

AVERAGE INDIVIDUAL DOSE-COMMITMENT HISTOGRAM FOR

\section{SAINT LUCIE}

Dose Commitments (person-rem) from Liquid Pathways

\begin{tabular}{|c|c|c|c|c|c|}
\hline & Tota l Body & $\underline{G I-L I I}$ & Thyroid & Bone & Liver \\
\hline $\begin{array}{l}\text { Infant } \\
\text { Child } \\
\text { Teen } \\
\text { Adult }\end{array}$ & $\begin{array}{l}0.0 \mathrm{E}+00 \\
5.5 \mathrm{E}-04 \\
5.0 \mathrm{E}-\mathrm{D} 4 \\
3.9 \mathrm{E}-03\end{array}$ & $\begin{array}{l}0.0 \mathrm{E}+00 \\
2.6 \mathrm{E}-03 \\
5.5 \mathrm{E}-03 \\
4.8 \mathrm{E}-02\end{array}$ & $\begin{array}{l}0.0 \mathrm{E}+00 \\
7.3 \mathrm{E}-04 \\
5.2 \mathrm{E}-04 \\
3.4 \mathrm{E}-03\end{array}$ & $\begin{array}{l}0.0 \mathrm{E}+00 \\
5.5 \mathrm{E}-04 \\
3.3 \mathrm{E}-04 \\
1.9 \mathrm{E}-03\end{array}$ & $\begin{array}{l}0.0 \mathrm{E}+00 \\
8.1 \mathrm{E}-04 \\
7.0 \mathrm{E}-04 \\
4.2 \mathrm{E}-03\end{array}$ \\
\hline TOTAL & $5.0 \mathrm{E}-03$ & $5.6 \mathrm{E}-02$ & $4.7 \mathrm{E}-03$ & $2.8 \mathrm{E}-03$ & $5.7 \mathrm{E}-03$ \\
\hline
\end{tabular}

Dose Commitments (person-rem) from Airborne Pathways Total Body GI-LII Thyroid Bone Liver Lung

$\begin{array}{lllllll}\text { Infant } & 1.0 \mathrm{E}-02 & 1.0 \mathrm{E}-02 & 1.2 \mathrm{E}-01 & 8.9 \mathrm{E}-03 & 1.1 \mathrm{E}-\mathrm{D} 2 & 1.1 \mathrm{E}-02 \\ \text { Child } & 1.2 \mathrm{E}-01 & 1.2 \mathrm{E}-01 & 7.6 \mathrm{E}-01 & 9.8 \mathrm{E}-02 & 1.2 \mathrm{E}-01 & 1.3 \mathrm{E}-01 \\ \text { Teen } & 8.9 \mathrm{E}-02 & 8.9 \mathrm{E}-02 & 3.5 \mathrm{E}-01 & 7.1 \mathrm{E}-02 & 8.9 \mathrm{E}-02 & 9.8 \mathrm{E}-02 \\ \text { Adult } & 5.3 \mathrm{E}-01 & 5.3 \mathrm{E}-01 & 1.4 \mathrm{E}-00 & 4.3 \mathrm{E}-01 & 5.3 \mathrm{E}-01 & 5.6 \mathrm{E}-01 \\ \text { TOTAL } & 7.5 \mathrm{E}-01 & 7.5 \mathrm{E}-01 & 2.7 \mathrm{E}+0 \mathrm{D} & 6.0 \mathrm{E}-01 & 7.6 \mathrm{E}-01 & 8.0 \mathrm{E}-01\end{array}$

Production/Consumption factors:

Produce: $<1 \quad$ Mi lk: $<1 \quad$ Meat: $<1$

FRACTION OF POPULATION RECEIVING AN AVERAGE INDIVIDUAL TOTAL-BOOY ODSE COMMITMENT FROM AIRBORNE PATHWAYS

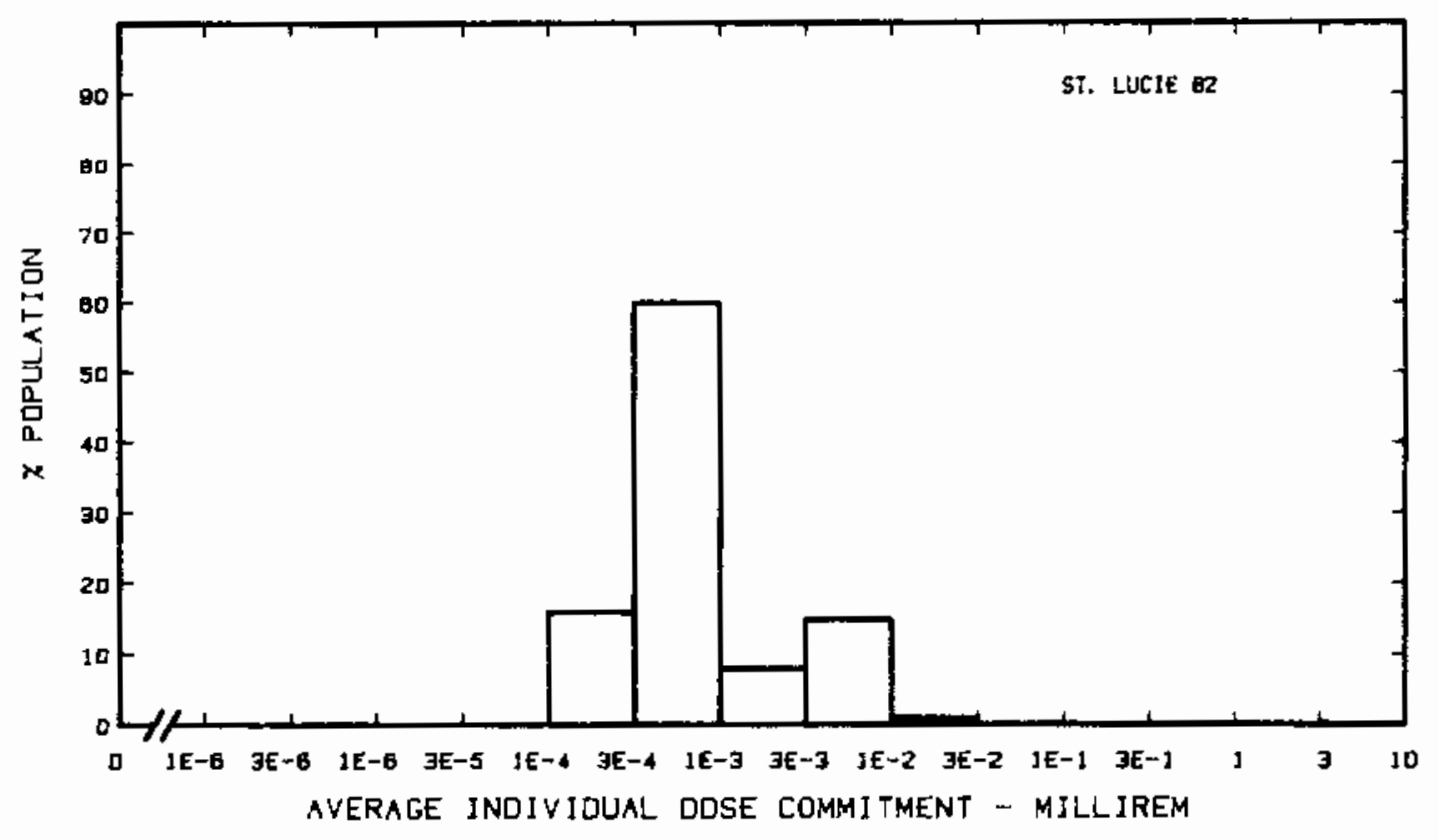


Site: SALEM

SALEM, NEW JERSEY

Location: $\quad$ N $39.4628^{\circ}$ W $75.5358^{\circ}$

POPULATION DATA

Total Population Within 2-to-80-km Region: 4.7E6

Major Metropolitan Centers Within Region:

Center

Population

Location

Philadelphia SMSA

$4,700,000$

400,000

Wilmington SMSA

130,000

Chester

46,000

Norristown

35,000

$63 \mathrm{~km} \quad \mathrm{NNE}$

$32 \mathrm{~km} \quad \mathrm{NNW}$

$48 \mathrm{~km} \quad \mathrm{E}$

$45 \mathrm{~km} \quad \mathrm{NNE}$

$74 \mathrm{~km} \mathrm{~N}$

SITE SPECIFIC DATA - AIRBORNE PATHWAYS

Average Annual State Production

of Crops and Animal Products

In 80-km Radius Circle

Veg: 7.4E7 kilogram

Milk: 2.7E8 liter

Meat: $2.4 E 7$ kilogram

Regional Productivity Factor:

0.9

Animal Grazing Factor:

0.6

Meteorology Period of Record: 1 JUN 70 - 31 MAY 71 Recovery: 95\%

SITE SPECIFIC DATA - WATERBORNE PATHWAYS via DELAWARE RIVER ESTUARY

Average River Flow

at Site: $16,000 \mathrm{ft}^{3} / \mathrm{s}$

Fish:

Edible Harvest: $3.6 \mathrm{E} 5 \mathrm{~kg} / \mathrm{yr}$ Dilution Factor: 1

Invertebrates: ${ }^{(a)}$

Edible Harvest: $1.6 E 5 \mathrm{~kg} / \mathrm{yr}$ Dilution Factor: 1

(a) Environment primarily salt water, so invertebrates considered in lieu of drinking water. 


\section{POPULATION DOSE-COMMITMENT ESTIMATES AND AVERAGE INDIVIDUAL DOSE-COMMITMENT HISTOGRAM FOR}

SALEM 1 AND 2

Dose Commitments (person-rem) from Liquid Pathways

\begin{tabular}{|c|c|c|c|c|c|}
\hline & Total Body & $G I-L I I$ & Thyroid & Bone & Liver \\
\hline $\begin{array}{l}\text { Infant } \\
\text { Child } \\
\text { Teen } \\
\text { Adult }\end{array}$ & $\begin{array}{l}0.0 \mathrm{E}+00 \\
4.4 \mathrm{E}-02 \\
3.1 \mathrm{E}-02 \\
2.0 \mathrm{E}-01\end{array}$ & $\begin{array}{l}0.0 \mathrm{E}+00 \\
1.2 \mathrm{E}-01 \\
2.5 \mathrm{E}-01 \\
2.2 \mathrm{E}+00\end{array}$ & $\begin{array}{l}0.0 \mathrm{E}+00 \\
3.2 \mathrm{E}-02 \\
2.2 \mathrm{E}-02 \\
1.5 \mathrm{E}-01\end{array}$ & $\begin{array}{l}0.0 \mathrm{E}+00 \\
9.1 \mathrm{E}-03 \\
5.4 \mathrm{E}-03 \\
3.2 \mathrm{E}-02\end{array}$ & $\begin{array}{l}0.0 \mathrm{E}+00 \\
3.0 \mathrm{E}-02 \\
2.7 \mathrm{E}-02 \\
1.7 \mathrm{E}-01\end{array}$ \\
\hline TOTAL & $2.8 \mathrm{E}-01$ & $2.6 \mathrm{E}+00$ & $2.0 \mathrm{E}-01$ & 4. $7 \mathrm{E}-02$ & $2.2 \mathrm{E}-01$ \\
\hline
\end{tabular}

Dose Commitments (person-rem) from Airborne Pathways Total Body GI-LII Thyroid Bone Liver Lung

$\begin{array}{lllllll}\text { Infant } & 3.8 \mathrm{E}-03 & 3.8 \mathrm{E}-03 & 1.1 \mathrm{E}-02 & 9.4 \mathrm{E}-04 & 3.8 \mathrm{E}-03 & 3.9 \mathrm{E}-03 \\ \text { Child } & 5.3 \mathrm{E}-02 & 5.3 \mathrm{E}-02 & 9.9 \mathrm{E}-02 & 1.0 \mathrm{E}-02 & 5.3 \mathrm{E}-02 & 5.5 \mathrm{E}-02 \\ \text { Teen } & 3.7 \mathrm{E}-02 & 3.7 \mathrm{E}-02 & 5.5 \mathrm{E}-02 & 7.5 \mathrm{E}-03 & 3.7 \mathrm{E}-02 & 3.9 \mathrm{E}-02 \\ \text { Adult } & 2.0 \mathrm{E}-01 & 2.1 \mathrm{E}-01 & 2.7 \mathrm{E}-01 & 4.6 \mathrm{E}-02 & 2.0 \mathrm{E}-01 & 2.1 \mathrm{E}-01 \\ \text { TOTAL } & 3.0 \mathrm{E}-01 & 3.0 \mathrm{E}-01 & 4.3 \mathrm{E}-01 & 6.4 \mathrm{E}-02 & 3.0 \mathrm{E}-01 & 3.1 \mathrm{E}-01\end{array}$

Production/Consumption factors:

Produce: $<1 \quad$ Milk: <1 $\quad$ Meat: <1

FRACTION DF PDPULATIDN RECEIVING AN AVERAGE INOIVIDUAL TOTAL-BODY DOSE COMMITMENT FROM AIRBDRNE PATHWAYS

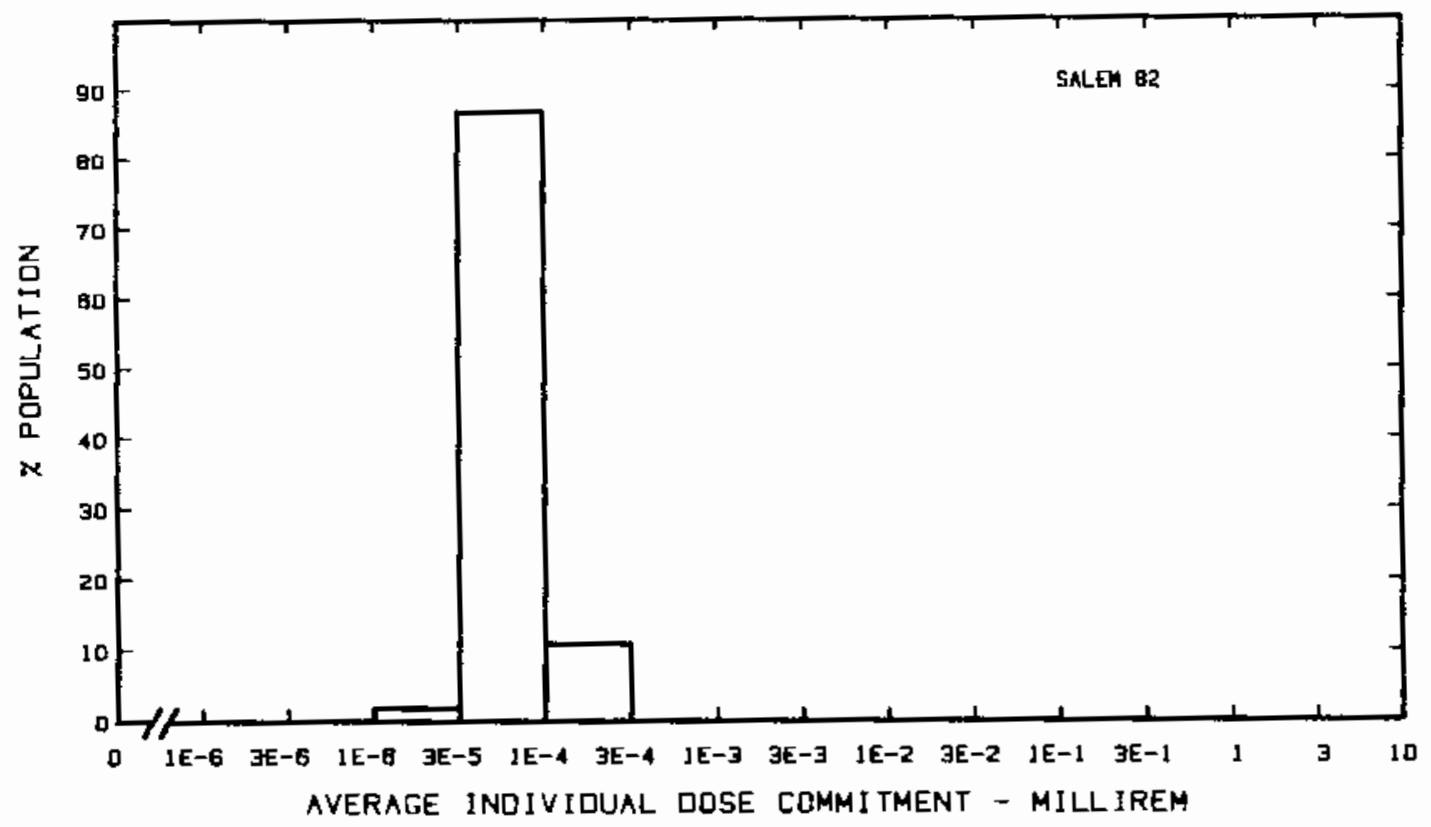


Site: SAN ONOFRE

Location:

W $\quad 117.5569^{\circ}$

POPULATION DATA

Total Population Within 2-to-80-km Region: 4.7E6

Major Metropolitan Centers Within Region:

Center Population Location

San Oiego SMSA

Anaheim-Santa Ana-Garden Grove SMSA 1,900,000 Long Beach

Huntington Beach

Riverside

Pomona

$1,900,000$

$68 \mathrm{~km} \quad \mathrm{SSE}$

$62 \mathrm{~km} \quad \mathrm{NW}$

$75 \mathrm{~km} \quad \mathrm{NW}$

$61 \mathrm{~km} \quad \mathrm{NW}$

170,000

170,000

94,000

$68 \mathrm{~km} \mathrm{~N}$

$79 \mathrm{~km} \quad \mathrm{NNW}$

SITE SPECIFIC DATA - AIRBORNE PATHWAYS

Average Annual State Production

of Crops and Animal Products

In $80-\mathrm{km}$ Radius Circle

Regional Productivity Factor:

Animal Grazing Factor:
Veg: $4.8 \mathrm{E} 7 \mathrm{ki}$ logram

Milk: 2.3E8 liter

Meat: $5.0 E 7$ kilogram

0.6

1

Meteorology Period of Record: 25 JAN $73-24$ JAN 76 Recovery: 88\%

SITE SPECIFIC OATA - WATERBORNE PATHWAYS via PACIFIC OCEAN

Average Dilution Flow

from Plant: $290 \mathrm{ft}^{3} / \mathrm{s}$

Fish:

Edible Harvest: $2.9 E 4^{(a)}$
Dilution Factor:

Invertebrates:

Edible Harvest: $2.9 g^{(a)} \mathrm{kg} / \mathrm{yr}$
Dilution Factor: 1

(a) Seafood caught in undiluted effluent (FES, 1973). 


\section{POPULATION DOSE-COMMITMENT ESTIMATES AND}

AVERAGE INDIV IDUAL DOSE-COMMITMENT HISTOGRAM FOR

SAN ONOFRE 1,2 , AND 3

Dose Commitments (person-rem) from Liquid Pathways

\begin{tabular}{|c|c|c|c|c|c|}
\hline & Total Body & GI-LII & Thyroid & Bone & Liver \\
\hline $\begin{array}{l}\text { Infant } \\
\text { Child } \\
\text { Teen } \\
\text { Adult }\end{array}$ & $\begin{array}{l}0.0 \mathrm{E}+00 \\
4.4 \mathrm{E}-02 \\
4.5 \mathrm{E}-02 \\
3.9 \mathrm{E}-01\end{array}$ & $\begin{array}{l}0.0 \mathrm{E}+00 \\
8.3 \mathrm{E}-02 \\
1.7 \mathrm{E}-01 \\
1.5 \mathrm{E}-00\end{array}$ & $\begin{array}{l}0.0 \mathrm{E}+00 \\
1.5 \mathrm{E}-03 \\
1.2 \mathrm{E}-03 \\
9.1 \mathrm{E}-03\end{array}$ & $\begin{array}{l}0.0 \mathrm{E}+00 \\
7.5 \mathrm{E}-02 \\
4.5 \mathrm{E}-02 \\
2.6 \mathrm{E}-01\end{array}$ & $\begin{array}{l}0.0 \mathrm{E}+00 \\
9.9 \mathrm{E}-02 \\
8.4 \mathrm{E}-02 \\
5.0 \mathrm{E}-01\end{array}$ \\
\hline TOTAL & $4.8 \mathrm{E}-01$ & $1.7 \mathrm{E}+00$ & $1.2 \mathrm{E}-02$ & $3.8 \mathrm{E}-01$ & $6.9 \mathrm{E}-01$ \\
\hline
\end{tabular}

Dose Commitments (person-rem) from Airborne Pathways

Total Body GI-LII Thyroid Bone Liver Lung

$\begin{array}{lllllll}\text { Infant } & 3.6 \mathrm{E}-04 & 3.6 \mathrm{E}-04 & 3.9 \mathrm{E}-04 & 5.1 \mathrm{E}-05 & 3.6 \mathrm{E}-04 & 3.7 \mathrm{E}-04 \\ \text { Child } & 5.5 \mathrm{E}-03 & 5.5 \mathrm{E}-03 & 5.6 \mathrm{E}-03 & 5.7 \mathrm{E}-04 & 5.5 \mathrm{E}-03 & 5.6 \mathrm{E}-03 \\ \text { Teen } & 4.0 \mathrm{E}-03 & 4.0 \mathrm{E}-03 & 4.0 \mathrm{E}-03 & 4.1 \mathrm{E}-04 & 4.0 \mathrm{E}-03 & 4.1 \mathrm{E}-03 \\ \text { Adult } & 2.3 \mathrm{E}-02 & 2.3 \mathrm{E}-02 & 2.3 \mathrm{E}-02 & 2.5 \mathrm{E}-03 & 2.3 \mathrm{E}-02 & 2.3 \mathrm{E}-02 \\ \text { TOTAL } & 3.3 \mathrm{E}-02 & 3.3 \mathrm{E}-02 & 3.3 \mathrm{E}-02 & 3.5 \mathrm{E}-03 & 3.3 \mathrm{E}-02 & 3.3 \mathrm{E}-02\end{array}$

Production/Consumption factors:

Produce: $<1 \quad$ Milk: $<1 \quad$ Meat: $<1$

FRACTION OF POPLLATION RECEIVING AN AVERAGE INDIVIDUAL TOTAL-BDOY DOSE CDMMITMENT FRDM AIRBORNE PATHWAYS

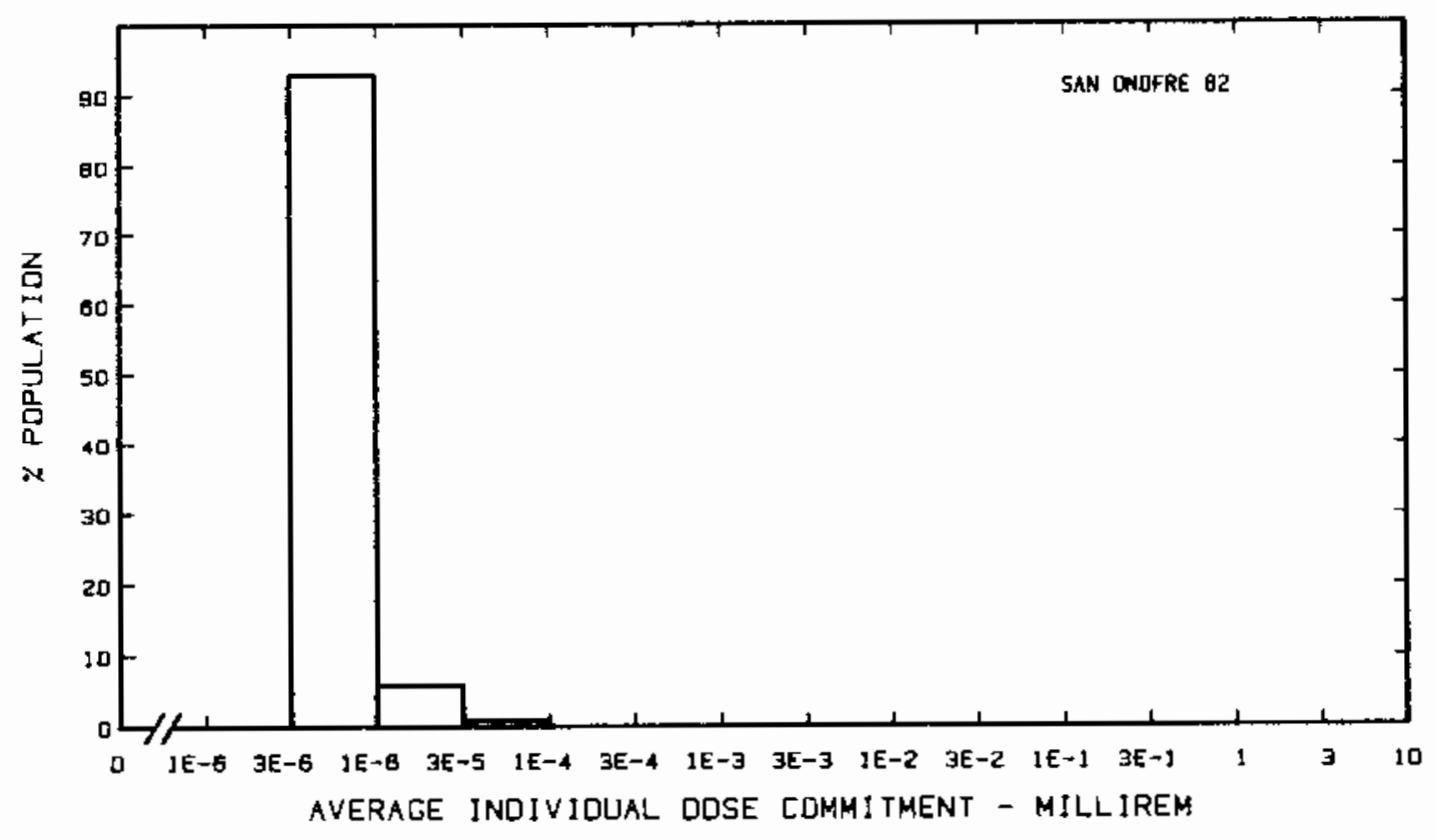


Site: SEQUOYAH

HAMILTON COUNTY, TENNESSEE

Location: $\quad \mathrm{N} 35.2233^{\circ} \quad$ W $85.0878^{\circ}$

POPULATION DATA

Total Population Within 2-to-80-km Region: 8.3E5

Major Metropolitan Centers Within Region:

Center

Chattanooga SMSA

Cleveland

East Ridge

Dalton

Athens

\begin{tabular}{c} 
Population \\
\hline 430,000 \\
26,000 \\
21,000 \\
21,000 \\
12,000
\end{tabular}

Location

$28 \mathrm{~km} \quad \mathrm{SW}$

$21 \mathrm{~km} \quad \mathrm{SE}$

$27 \mathrm{~km} \quad \mathrm{SSW}$

$50 \mathrm{~km} \mathrm{~S}$

$53 \mathrm{~km}$ ENE

SITE SPECIFIC DATA - AIRBORNE PATHWAYS

Average Annual State Production

of Crops and Animal Products

In 80-km Radius Circle

Regional Productivity Factor:

Animal Grazing Factor:
Veg: $1.1 E 7$ kilogram

Milk: 1.6E8 liter

Meat: $1.2 \mathrm{E} 8$ kilogram

0.25

0.7

Meteorology Period of Record: 1 JAN 72 - 31 DEC 75 Recovery: 93\%

SITE SPECIFIC DATA - WATERBORNE PATHWAYS via TENNESSEE RIVER

Average River Flow

at Site: $35,000 \mathrm{ft}^{3} / \mathrm{s}$

Drinking Water:

Exposed Population: 430,000

Dilution Factor: 1

Fish:

Edible Harvest: $1.7 \mathrm{E5}{ }^{(\mathrm{a})} \mathrm{kg} / \mathrm{yr}$

Dilution Factor: 1

(a) Catch data given in FES (1974). 
POPULATION OOSE-COMMITMENT ESTIMATES AND

AVERAGE INOIVIDUAL OOSE-COMMITMENT HISTOGRAM FOR

SEQUOYAH

Dose Commitments (person-rem) from Liquid Pathways

Total Body GI-LII Thyroid Bone Liver

$\begin{array}{llllll}\text { Infant } & 1.4 \mathrm{E}-02 & 1.3 \mathrm{E}-02 & 5.4 \mathrm{E}-02 & 1.3 \mathrm{E}-02 & 1.4 \mathrm{E}-02 \\ \text { Child } & 1.6 \mathrm{E}-01 & 1.8 \mathrm{E}-01 & 4.2 \mathrm{E}-01 & 1.6 \mathrm{E}-01 & 1.8 \mathrm{E}-01 \\ \text { Teen } & 6.5 \mathrm{E}-02 & 1.2 \mathrm{E}-01 & 1.4 \mathrm{E}-01 & 5.3 \mathrm{E}-02 & 7.8 \mathrm{E}-02 \\ \text { Adult } & 5.6 \mathrm{E}-01 & 1.1 \mathrm{E}+00 & 1.0 \mathrm{E}+00 & 3.3 \mathrm{E}-01 & 5.8 \mathrm{E}-01 \\ \text { TOTAL } & 8.0 \mathrm{E}-01 & 1.4 \mathrm{E}+00 & 1.6 \mathrm{E}+00 & 5.7 \mathrm{E}-01 & 8.4 \mathrm{E}-01\end{array}$

Dose Commitments (person-rem) from Airborne Pathways

Total Body GI-LII Thyroid Bone Liver Lung

$\begin{array}{lllllll}\text { Infant } & 5.9 \mathrm{E}-03 & 5.9 \mathrm{E}-03 & 6.1 \mathrm{E}-03 & 4.8 \mathrm{E}-03 & 5.9 \mathrm{E}-03 & 6.8 \mathrm{E}-03 \\ \text { Child } & 7.2 \mathrm{E}-02 & 7.3 \mathrm{E}-02 & 7.4 \mathrm{E}-02 & 5.3 \mathrm{E}-02 & 7.2 \mathrm{E}-02 & 8.6 \mathrm{E}-02 \\ \text { Teen } & 5.4 \mathrm{E}-02 & 5.4 \mathrm{E}-02 & 5.4 \mathrm{E}-02 & 3.9 \mathrm{E}-02 & 5.3 \mathrm{E}-02 & 6.8 \mathrm{E}-02 \\ \text { Adult } & 3.2 \mathrm{E}-01 & 3.3 \mathrm{E}-01 & 3.3 \mathrm{E}-01 & 2.3 \mathrm{E}-01 & 3.2 \mathrm{E}-01 & 3.7 \mathrm{E}-01 \\ \text { TOTAL } & 4.5 \mathrm{E}-01 & 4.6 \mathrm{E}-01 & 4.6 \mathrm{E}-01 & 3.3 \mathrm{E}-01 & 4.5 \mathrm{E}-01 & 5.4 \mathrm{E}-01\end{array}$

Production/Consumption factors:

Produce: $<1 \quad$ Milk: $<1 \quad$ Meat: $<1$

FRACTION DF PDPULATION RECEIVING AN AVERAGE INDIVIOUAL TOTAL-BOQY DOSE COMMITMENT FROM AIRBORNE. PATHWAYS

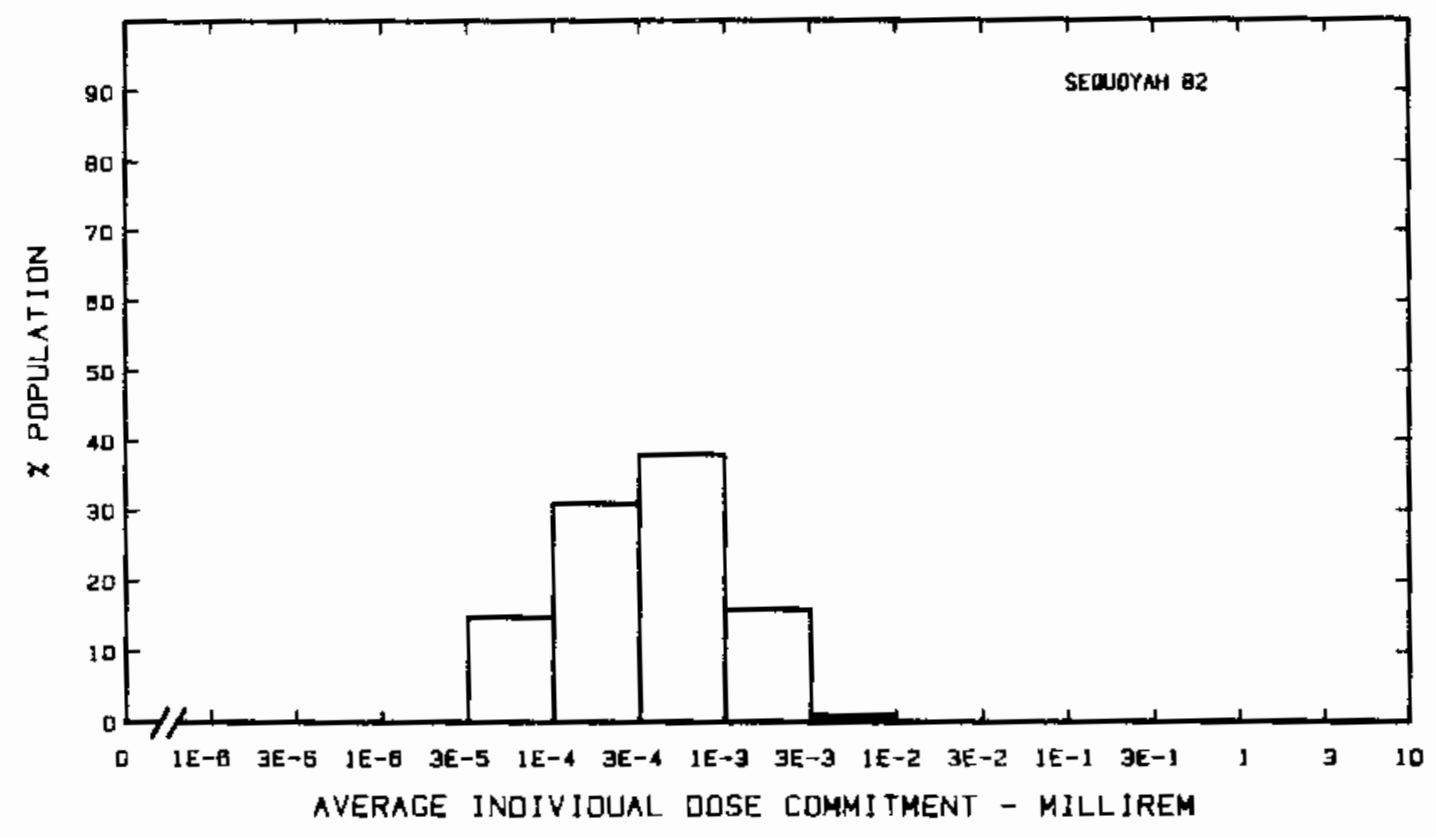


Site: SUMMER

JENKINSVILLE, SOUTH CAROLINA

Location: $\quad$ N $34.2958^{\circ} \quad$ W $81.3203^{\circ}$

POPULATION DATA

Total Population Within 2-to-80-km Region: 8.2E5

Major Metropolitan Centers Within Region:

Center

Columbia SMSA

Rock Hill

Greenwood

Union

Laurens
Population

410,000

35,000

22,000

11,000

11,000
Location

$42 \mathrm{~km} \quad \mathrm{SE}$

$75 \mathrm{~km} \quad \mathrm{NNE}$

$78 \mathrm{~km} \quad \mathrm{~W}$

$54 \mathrm{~km} \quad \mathrm{NNW}$

$68 \mathrm{~km} \quad \mathrm{WNW}$

SITE SPECIFIC OATA - AIRBORNE PATHWAYS

Average Annual State Production

of Crops and Animal Products

In 80-km Radius Circle

Regional Productivity Factor:

Animal Grazing Factor:

Meteorology Period of Record: 1 Jan 75 - 31 Dec 75
Veg: $\quad 7.4 E 6 \mathrm{kilog}$ rari

Milk: $5.7 E 7$ liter

Meat: $5.0 E 7$ kilogram

0.9

0.7

SITE SPECIFIC DATA - WATERBORNE PATHWAYS via PARR RES, and BROAO RIVER

Average River $\mathrm{Flow}$

at Site: $6,000 \mathrm{ft}^{3} / \mathrm{s}$

Drinking Water:

Exposed Population: 120,000

0ilution Factor:

Fish:

Edible Harvest: $2.2 \mathrm{~kg} / \mathrm{yr}^{(\mathrm{a})}$

0ilution Factor: 1

(a) Average individual consumption rate as given in the FES (1973) used in lieu of catch data. 
POPULATION DOSE-COMMITMENT ESTIMATES AND

AVERAGE INDIVIDUAL DOSE-COMMITMENT HISTDGRAM FOR

SUMMER

Dose Commitments (person-rem) from Liquid Pathways

\begin{tabular}{|c|c|c|c|c|c|}
\hline & Total Body & GI-LII & Thyroid & Bone & Liver \\
\hline $\begin{array}{l}\text { Infant } \\
\text { Child } \\
\text { Teen } \\
\text { Adult }\end{array}$ & $\begin{array}{l}5.5 E-06 \\
6.3 E-05 \\
2.5 E-05 \\
2.1 E-04\end{array}$ & $\begin{array}{l}5.5 \mathrm{E}-06 \\
6.4 \mathrm{E}-05 \\
2.6 \mathrm{E}-05 \\
2.2 \mathrm{E}-04\end{array}$ & $\begin{array}{l}9.8 \mathrm{E}-06 \\
9.8 \mathrm{E}-05 \\
3.7 \mathrm{E}-05 \\
3.0 \mathrm{E}-04\end{array}$ & $\begin{array}{l}3.8 \mathrm{E}-08 \\
3.6 \mathrm{E}-07 \\
1.0 \mathrm{E}-07 \\
6.4 \mathrm{E}-07\end{array}$ & $\begin{array}{l}5.5 \mathrm{E}-06 \\
6.3 \mathrm{E}-05 \\
2.5 \mathrm{E}-05 \\
2.1 \mathrm{E}-04\end{array}$ \\
\hline TOTAL & 3. DE-04 & 3. $2 \mathrm{E}-04$ & $4.4 E-04$ & I. $1 \mathrm{E}-06$ & $3.0 E-04$ \\
\hline
\end{tabular}

Dose Commitments (person-rem) from Airborne Pathways Total Body GI-LII Thyroid Bone Liver Lung

$\begin{array}{lllllll}\text { Infant } & 2.9 \mathrm{E}-06 & 2.9 \mathrm{E}-06 & 2.9 \mathrm{E}-06 & 2.9 \mathrm{E}-06 & 2.9 \mathrm{E}-06 & 7.8 \mathrm{E}-06 \\ \text { Child } & 3.2 \mathrm{E}-05 & 3.2 \mathrm{E}-05 & 3.2 \mathrm{E}-05 & 3.2 \mathrm{E}-05 & 3.2 \mathrm{E}-05 & 1.0 \mathrm{E}-04 \\ \text { Teen } & 2.4 \mathrm{E}-05 & 2.4 \mathrm{E}-05 & 2.4 \mathrm{E}-05 & 2.4 \mathrm{E}-05 & 2.4 \mathrm{E}-05 & 1.1 \mathrm{E}-04 \\ \text { Adult } & 1.4 \mathrm{E}-04 & 1.4 \mathrm{E}-04 & 1.4 \mathrm{E}-04 & 1.4 \mathrm{E}-04 & 1.4 \mathrm{E}-04 & 4.3 \mathrm{E}-04 \\ \text { TOTAL } & 2.0 \mathrm{E}-04 & 2.0 \mathrm{E}-04 & 2.0 \mathrm{E}-04 & 2.0 \mathrm{E}-04 & 2.0 \mathrm{E}-04 & 6.5 \mathrm{E}-04\end{array}$

Production/Consumption factors:

Produce: $<1 \quad$ Milk: $<1 \quad$ Meat: $<1$

FRACTION OF POPULATION RECEIVING AN AVERAGE INDIVIDUAL TOTAL-BOOY DOSE COMMITMENT FRDM AIRGORNE PATHWAYS

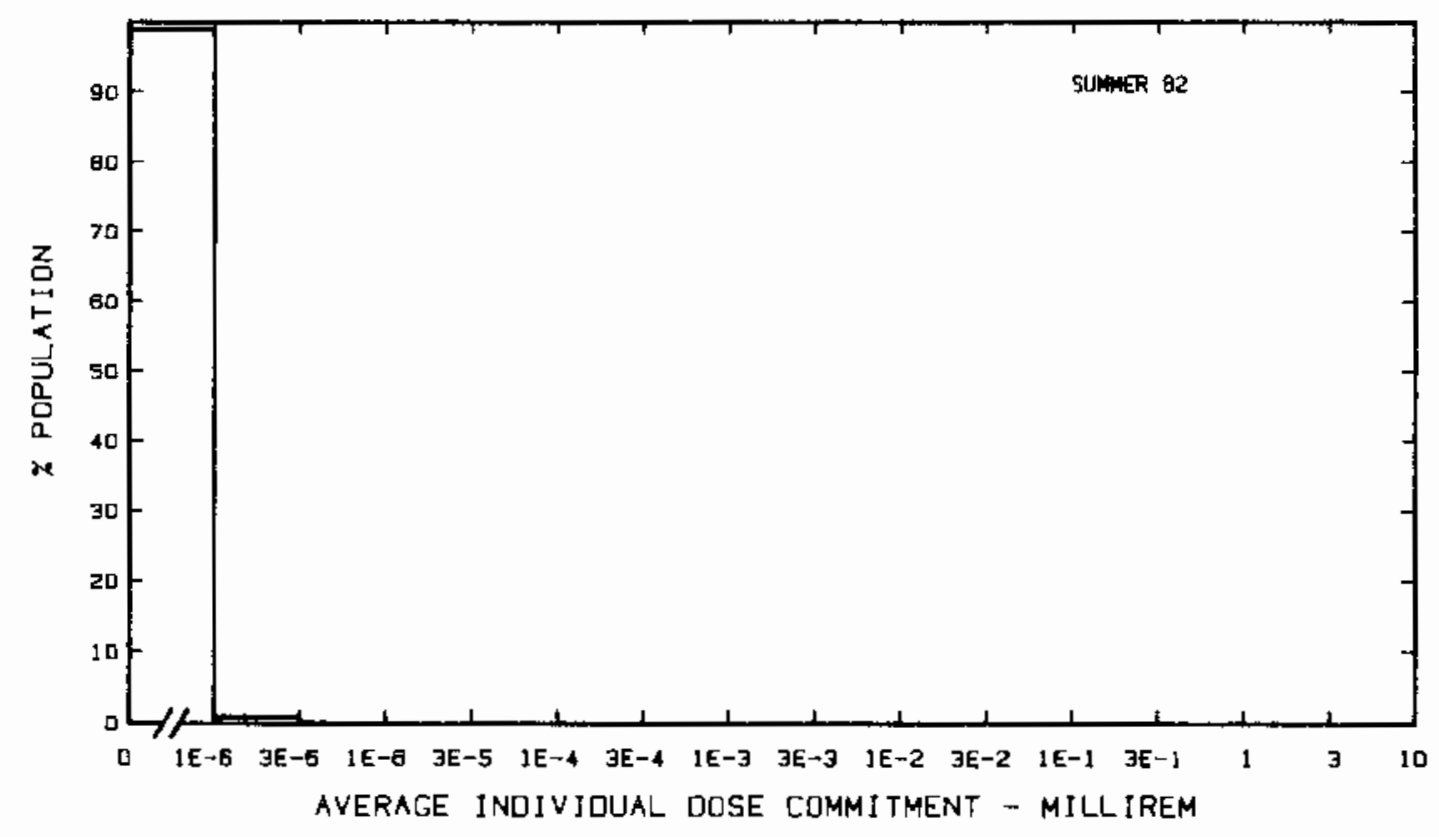


Site: SURRY

SURRY COUNTY, VIRGINIA

Location: N $37.1656^{\circ} \quad$ W $76.6983^{\circ}$

POPULATION DATA

Total Population Within 2-to-80-km Region: 1.7E6

Major Metropolitan Centers Within Region:

Center

Population

Location

Norfolk-Virginia Beach-Portsmouth SMSA Richmond SMSA

820,000

630,000

Newport News-Hampton SMSA

360,000

Petersburg-Colonial Heights-Hopewell SMSA 130,000

Williamsburg

10,000

$50 \mathrm{~km}$

$77 \mathrm{~km}$

$33 \mathrm{~km}$

$63 \mathrm{~km}$

$12 \mathrm{~km} \quad \mathrm{~N}$

SITE SPECIFIC DATA - AIRBORNE PATHWAYS

Average Annual State Production

of Crops and Animal Products

In 80-km Radius Circle

Regional Productivity Factor:

Animal Grazing Factor:

Meteorology Period of Record: 3 MAR 74 - 2 MAR 75 Recovery: 91\%

Veg: $3.5 \mathrm{E} 7 \mathrm{kilogram}$

Milk: $1.5 \mathrm{E} 8$ liter

Meat: $7.4 E 7$ kilogram

0.8

0.7

SITE SPECIFIC DATA - WATERBORNE PATHWAYS via JAMES RIVER ESTUARY

Average River Flow
at Site: $25,000 \mathrm{ft}^{3} / \mathrm{s}$ (a)

Fish:

Edible Harvest: $6.0 \mathrm{E} 5 \mathrm{~kg} / \mathrm{yr}$ Dilution Factor: 1

Invertebrates: ${ }^{(b)}$

Edible Harvest: $1.1 \mathrm{E} 6 \mathrm{~kg} / \mathrm{yr}$

Dilution Factor: 1

(a) Flow includes fresh water river flow and saline "mixing flow" of estuary (FES, 1972).

(b) Environment primarily salt water, so invertebrates considered in 1 ieu of drinking water. 
POPULATION DOSE-COMMITMENT ESTIMATES AND

AVERAGE INDIVIDUAL DOSE-COMMITMENT HISTOGRAM FOR

SURRY 1 AND 2

Dose Commitments (person-rem) from Liquid Pathways

Total Body GI-LII Thyroid Bone Liver

$\begin{array}{llllll}\text { Infant } & 0.0 \mathrm{E}+00 & 0.0 \mathrm{E}+00 & 0.0 \mathrm{E}+00 & 0.0 \mathrm{E}+00 & 0.0 \mathrm{E}+00 \\ \text { Child } & 1.1 \mathrm{E}-01 & 1.3 \mathrm{E}-01 & 1.1 \mathrm{E}+00 & 2.1 \mathrm{E}-01 & 2.7 \mathrm{E}-01 \\ \text { Teen } & 1.2 \mathrm{E}-01 & 2.7 \mathrm{E}-01 & 7.6 \mathrm{E}-01 & 1.2 \mathrm{E}-01 & 2.2 \mathrm{E}-01 \\ \text { Adult } & 1.1 \mathrm{E}+01 & 2.4 \mathrm{E}+00 & 5.0 \mathrm{E}+00 & 7.1 \mathrm{E}-01 & 1.3 \mathrm{E}+01 \\ \text { TOTAL } & 1.4 \mathrm{E}+00 & 2.8 \mathrm{E}+00 & 6.8 \mathrm{E}+00 & 1.0 \mathrm{E}+00 & 1.8 \mathrm{E}+00\end{array}$

Dose Commitments (person-rem) from Airborne Pathways

Total Body GI-LII Thyroid Bone Liver Lung

Infant $1.2 \mathrm{E}-02 \quad 1.2 \mathrm{E}-02 \quad 5.0 \mathrm{E}-02 \quad 1.2 \mathrm{E}-02 \quad 1.2 \mathrm{E}-02 \quad 1.3 \mathrm{E}-02$

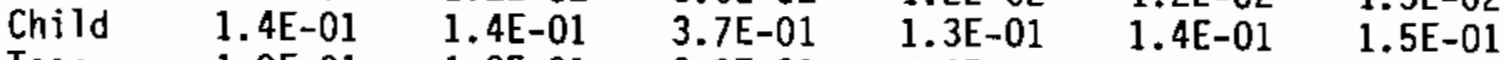

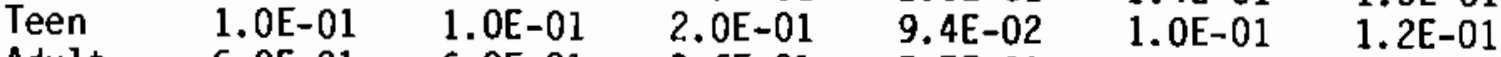

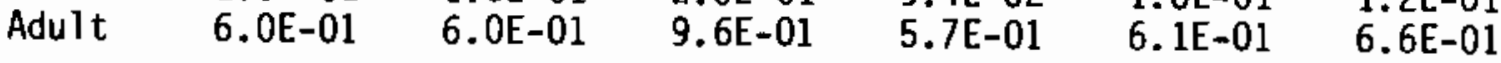

TOTAL $\quad 8.6 \mathrm{E}-01 \quad 8.5 \mathrm{E}-01 \quad 1.6 \mathrm{E}+00 \quad 8.1 \mathrm{E}-01 \quad 8.6 \mathrm{E}-01 \quad 9.5 \mathrm{E}-01$

Production/Consumption factors:

Produce: $<1 \quad$ Milk: $<1 \quad$ Meat: $<1$

FRACTIDN DF POPULATION RECEIVING AN AVERAGE INOIVIDUAL TOTAL-BODY DDSE COMMITMENT FROM AIRBDRNE PATHWAYS

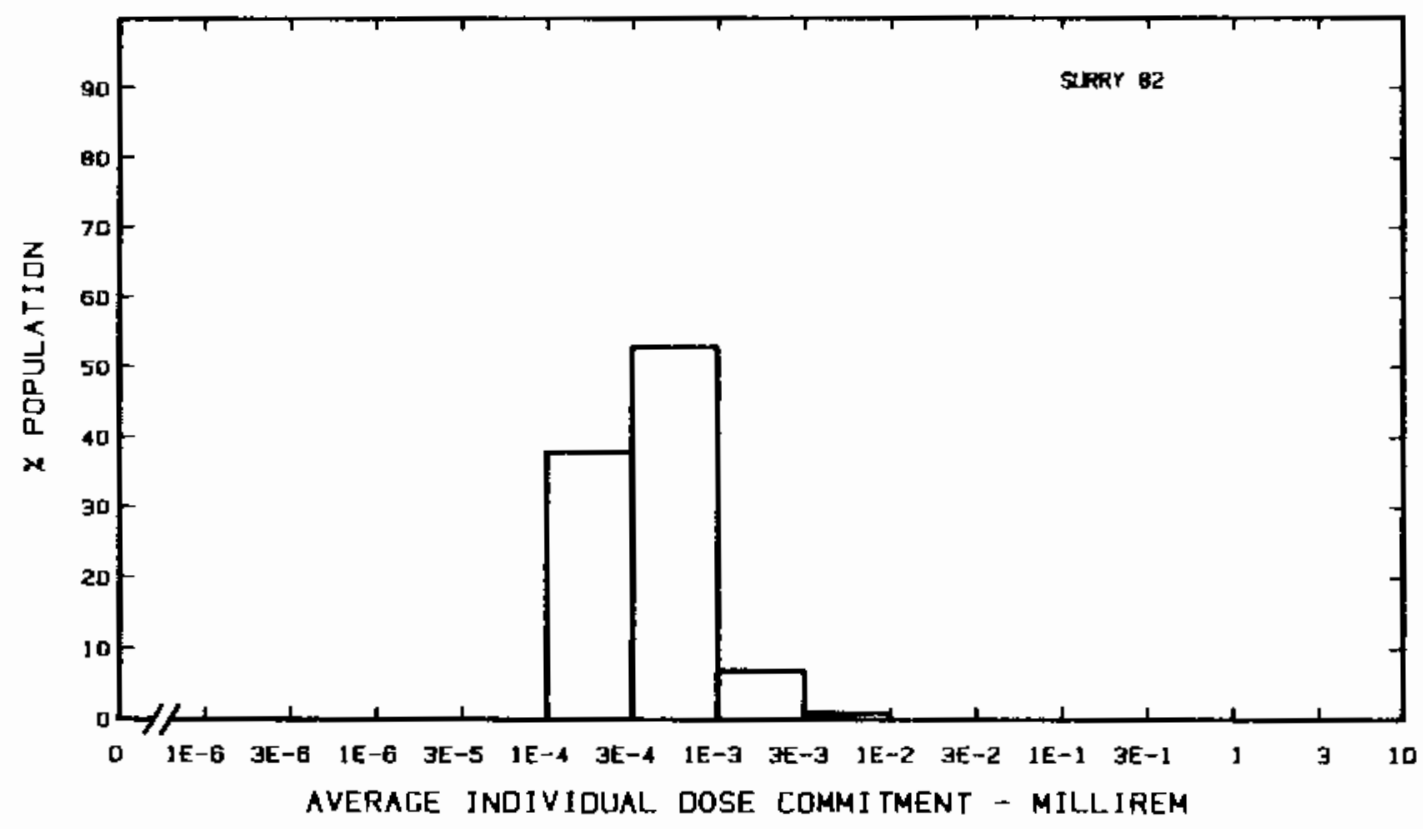


Site: SUSQUEHANNA

BERWICK, PENNSYLVANIA

Location:

N $41.10^{\circ}$

W $76.15^{\circ}$

POPULATION DATA

Total Population Within 2-to-80-km Region: 1.5 E6

Major Metropolitan Centers Within Region:

\begin{tabular}{lcccc} 
Center & Population & & \multicolumn{2}{c}{ Location } \\
\cline { 2 - 6 } Williamsport - SMSA & 118,000 & & $73 \mathrm{~km}$ & WNW \\
Allentown & 100,000 & $79 \mathrm{~km}$ & SE \\
Scranton & 88,000 & $53 \mathrm{~km}$ & $\mathrm{NE}$ \\
Wilkes-Barre & 52,000 & $28 \mathrm{~km}$ & $\mathrm{NE}$ \\
Hazleton & 27,000 & $21 \mathrm{~km}$ & SE
\end{tabular}

SITE SPECIFIC DATA - AIRBORNE PATHWAYS

Average Annual State Production

of Crops and Animal Products

In 80-km Radius Circle

Veg: $5.3 \mathrm{E} 7$ kilogram

Milk: $5.3 E 8$ liter

Meat: $5.4 \mathrm{E} 7 \mathrm{ki}$ logram

Regional Productivity Factor:

Animal Grazing Factor:

Meteorology Period of Record: 1 Jan $75-31$ Dec 75 Recovery: 98\%

SITE SPECIFIC DATA - WATERBORNE PATHWAYS via SUSQUEHANNA RIVER

Average River Flow

at Site: $13,000 \mathrm{ft}^{3} / \mathrm{s}$

Drinking Water:

Exposed Population: 100,000

Dilution Factor: 1

Fish:

Edible Harvest: (a)

Difution Factor: 0.1 (b)

(a) No fish catch data given in FES, so generic consumption rates used (Table A.1).

(b) Ten percent of population consumes fish from river. 
POPULATION DOSE-COMMITMENT ESTIMATES AND

AVERAGE INDIVIDUAL DOSE-COMMITMENT HISTOGRAM FOR

SUSQUEHANNA

Dose Commitments (person-rem) from Liquid Pathways

\begin{tabular}{|c|c|c|c|c|c|}
\hline & Total Body & GI-LII & Thyroid & Bone & Liver \\
\hline $\begin{array}{l}\text { Infant } \\
\text { Child } \\
\text { Teen } \\
\text { Adult }\end{array}$ & $\begin{array}{l}3.9 \mathrm{E}-05 \\
7.3 \mathrm{E}-03 \\
1.2 \mathrm{E}-02 \\
1.3 \mathrm{E}-01\end{array}$ & $\begin{array}{l}6.9 \mathrm{E}-05 \\
1.7 \mathrm{E}-03 \\
2.1 \mathrm{E}-03 \\
1.9 \mathrm{E}-02\end{array}$ & $\begin{array}{l}\text { 4. BE-04 } \\
3.7 \mathrm{E}-03 \\
1.3 \mathrm{E}-03 \\
9.0 \mathrm{E}-03\end{array}$ & $\begin{array}{l}1.2 \mathrm{E}-04 \\
2.2 \mathrm{E}-02 \\
1.3 \mathrm{E}-02 \\
7.8 \mathrm{E}-02\end{array}$ & $\begin{array}{l}1.5 \mathrm{E}-04 \\
3.1 \mathrm{E}-02 \\
2.7 \mathrm{E}-02 \\
1.6 \mathrm{E}-01\end{array}$ \\
\hline TOTAL & $1.5 \mathrm{E}-01$ & $2.2 E-02$ & 1. $4 \mathrm{E}-02$ & $1.1 \mathrm{E}-01$ & 2. $2 \mathrm{E}-01$ \\
\hline
\end{tabular}

Dose Commitments (person-rem) from Airborne Pathways

Total Body GI-LII Thyroid Bone Liver Lung

$\begin{array}{lllllll}\text { Infant } & 7.9 \mathrm{E}-04 & 8.0 \mathrm{E}-04 & 7.8 \mathrm{E}-04 & 5.3 \mathrm{E}-04 & 8.1 \mathrm{E}-04 & 8.1 \mathrm{E}-04 \\ \text { Child } & 9.6 \mathrm{E}-03 & 9.6 \mathrm{E}-03 & 9.5 \mathrm{E}-03 & 5.9 \mathrm{E}-03 & 9.7 \mathrm{E}-03 & 9.9 \mathrm{E}-03 \\ \text { Teen } & 6.6 \mathrm{E}-03 & 6.7 \mathrm{E}-03 & 6.6 \mathrm{E}-03 & 4.2 \mathrm{E}-03 & 6.7 \mathrm{E}-03 & 7.1 \mathrm{E}-03 \\ \text { Adult } & 3.9 \mathrm{E}-02 & 3.9 \mathrm{E}-02 & 3.8 \mathrm{E}-02 & 2.5 \mathrm{E}-02 & 3.9 \mathrm{E}-02 & 4.0 \mathrm{E}-02 \\ \text { TOTAL } & 5.6 \mathrm{E}-02 & 5.6 \mathrm{E}-02 & 5.5 \mathrm{E}-02 & 3.6 \mathrm{E}-02 & 5.6 \mathrm{E}-02 & 5.8 \mathrm{E}-02\end{array}$

Production/Consumption factors:

Produce: <1 Milk: $2.4 \quad$ Meat: <1

FRACTION OF POPULATION RECEIVING AN AVERACE INDIVIDUAL. TOTAL-BOOY DOSE COMMITMENT FROM AIRBORNE PATHWAYS

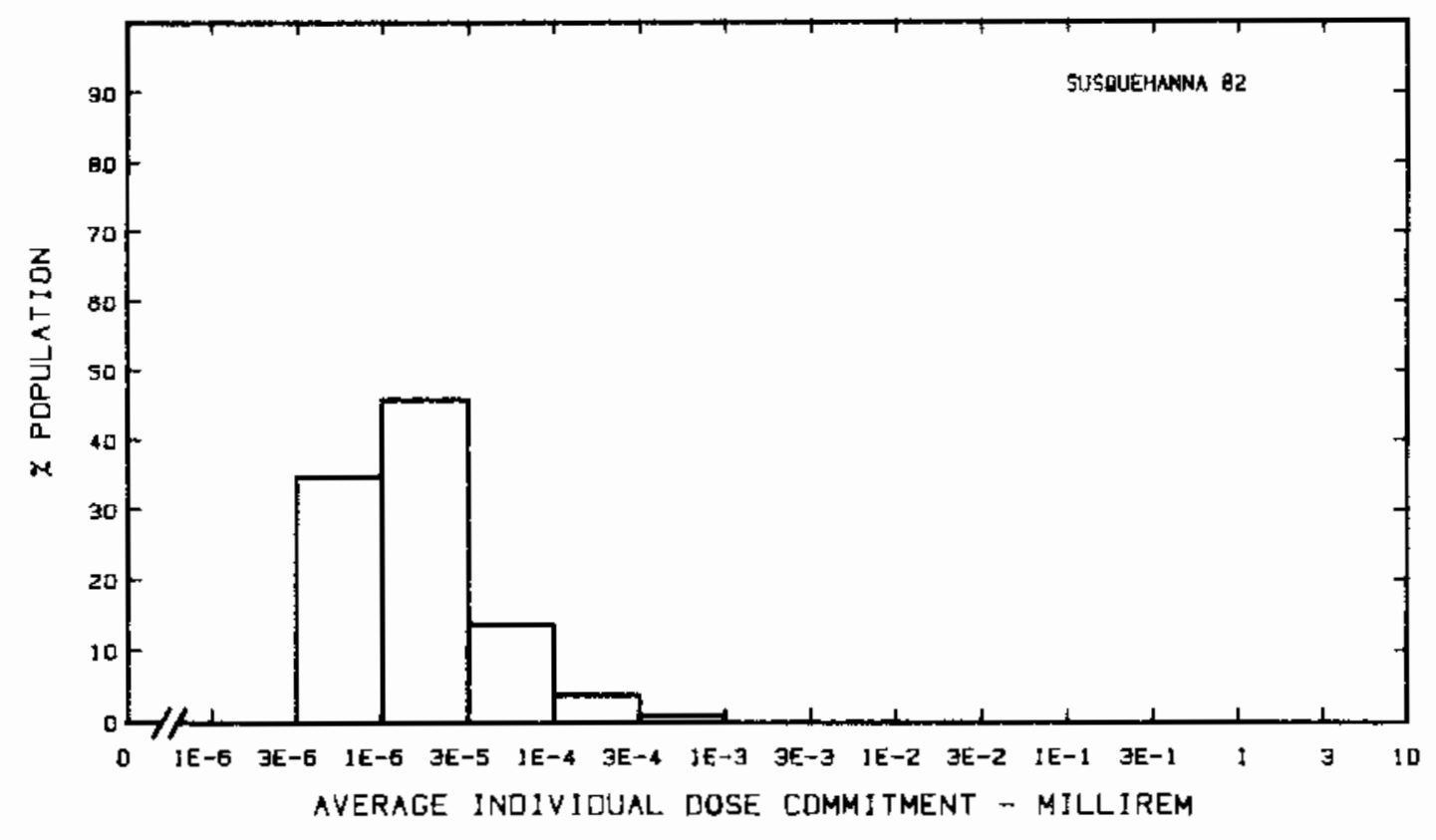


Site: THREE MILE ISLAND
Location:
N $40.1531^{\circ}$

POPULATION OATA

Total Population Within 2-to-80-km Region: 2.1E6

Major Metropolitan Centers Within Region:

\begin{tabular}{lcccc}
\multicolumn{1}{c}{ Center } & Population & \multicolumn{2}{c}{ Location } \\
\cline { 3 - 6 } Harrisburg SMSA & 450,000 & & \\
York SMSA & 380,000 & $21 \mathrm{~km}$ & $\mathrm{NW}$ \\
Lancaster SMSA & 360,000 & $38 \mathrm{~km}$ & ESE \\
Reading SMSA & 310,000 & $71 \mathrm{~km}$ & ENE \\
Lebanon & 26,000 & $33 \mathrm{~km}$ & $\mathrm{NE}$
\end{tabular}

SITE SPECIFIC DATA - AIRBORNE PATHWAYS

Average Annual State Production

of Crops and Animal Products

In 80-km Radius Circle

Regional Productivity Factor:

Animal Grazing Factor:
Veg: $5.3 \mathrm{E} 7 \mathrm{kilogram}$

Mi1k: 5.3E8 liter

Meat: $5.4 \mathrm{E} 7$ kilogram

1

0.5

Meteorology Period of Record: 1 OCT 72 - 30 SEP 73 Recovery: $80 \%$

SITE SPECIFIC DATA - WATERBORNE PATHWAYS via SUSQUEHANNA RIVER

Average River Flow

at Site: $34,000 \mathrm{ft}^{3} / \mathrm{s}$

Drinking Water: $\quad$ Exposed Population: 230,000

Dilution Factor: 1

Fish:

Edible Harvest: (a) $\mathrm{kg}(\mathrm{fr}$
Dilution Factor: 0.025

(a) No fish catch data given in FES, so generic consumption rates used (Table A-1).

(b) Ten percent of population consumes $25 \%$ of their fish from river (FES, 1972). 
POPULATION DOSE-COMMITMENT ESTIMATES AND AVERAGE INDIVIDUAL DOSE-COMMITMENT HISTOGRAM FOR

THREE MILE ISLAND 1 AND 2

Dose Commitments (person-rem) from Liquid Pathways

\begin{tabular}{|c|c|c|c|c|c|}
\hline & Total Body & GI-LII & Thyroid & Bone & Liver \\
\hline $\begin{array}{l}\text { Infant } \\
\text { Child } \\
\text { Teen } \\
\text { Adult }\end{array}$ & $\begin{array}{l}8.2 \mathrm{E}-05 \\
4.4 \mathrm{E}-03 \\
6.9 \mathrm{E}-03 \\
7.6 \mathrm{E}-\mathrm{D} 2\end{array}$ & $\begin{array}{l}2.7 \mathrm{E}-05 \\
4.5 \mathrm{E}-04 \\
3.9 \mathrm{E}-04 \\
3.3 \mathrm{E}-03\end{array}$ & $\begin{array}{l}2.3 \mathrm{E}-05 \\
2.5 \mathrm{E}-04 \\
9.7 \mathrm{E}-05 \\
8.3 \mathrm{E}-04\end{array}$ & $\begin{array}{l}5.1 \mathrm{E}-04 \\
2.4 \mathrm{E}-\mathrm{D} 2 \\
1.3 \mathrm{E}-02 \\
7.4 \mathrm{E}-02\end{array}$ & $\begin{array}{l}6.0 \mathrm{E}-04 \\
2.5 \mathrm{E}-02 \\
1.8 \mathrm{E}-02 \\
1.1 \mathrm{E}-01\end{array}$ \\
\hline TOTAL & $8.7 E-02$ & 4. $2 \mathrm{E}-03$ & $1.2 \mathrm{E}-03$ & 1. $1 E-01$ & $1.5 E-01$ \\
\hline
\end{tabular}

Dose Commitments (person-rem) from Airborne Pathways Total Body GI-LII Thyroid Bone Liver Lung

$\begin{array}{lllllll}\text { Infant } & 2.4 \mathrm{E}-03 & 2.4 \mathrm{E}-03 & 2.4 \mathrm{E}-\mathrm{D} 3 & 1.6 \mathrm{E}-04 & 2.5 \mathrm{E}-03 & 2.6 \mathrm{E}-03 \\ \text { Child } & 3.4 \mathrm{E}-02 & 3.4 \mathrm{E}-02 & 3.4 \mathrm{E}-02 & 1.8 \mathrm{E}-03 & 3.4 \mathrm{E}-02 & 3.7 \mathrm{E}-02 \\ \text { Teen } & 2.3 \mathrm{E}-02 & 2.3 \mathrm{E}-02 & 2.3 \mathrm{E}-02 & 1.2 \mathrm{E}-\mathrm{D} 3 & 2.3 \mathrm{E}-02 & 2.7 \mathrm{E}-02 \\ \text { Aduit } & 1.3 \mathrm{E}-01 & 1.3 \mathrm{E}-01 & 1.3 \mathrm{E}-01 & 7.0 \mathrm{E}-03 & 1.3 \mathrm{E}-01 & 1.4 \mathrm{E}-01 \\ \text { TOTAL } & 1.9 \mathrm{E}-01 & 1.9 \mathrm{E}-01 & 1.9 \mathrm{E}-01 & 1.0 \mathrm{E}-02 & 1.9 \mathrm{E}-01 & 2.0 \mathrm{E}-01\end{array}$

Production/Consumption factors:

Produce: <1 Milk: $2.0 \quad$ Meat: $<1$

FRACTION OF POPULATION RECEIVING AN AVERAGE INDIVIDUAL TOTAL-BODY DOSE COMMITMENT FROM AIRBDRNE PATHWAYS

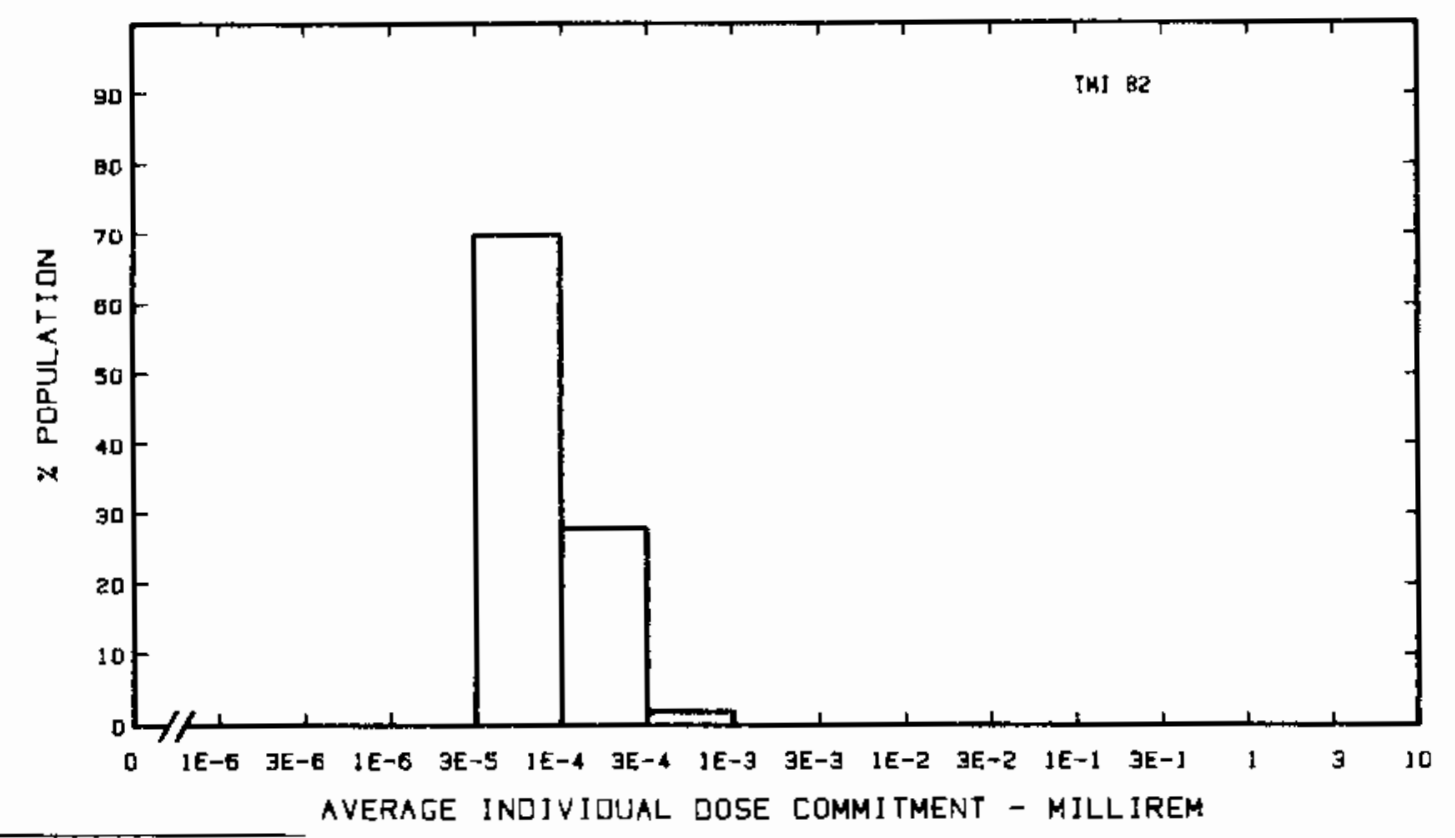

Note: Doses include releases from the TMI 2 / Epicore. 
Site: TROJAN

PRESCOTT, OREGON

Location: $\quad$ N $46.0408^{\circ} \quad$ W $122.8844^{\circ}$

POPULATION DATA

Total Population Within 2-to-80-km Region: 1.4E6

Major Metropolitan Centers Within Region:

\begin{tabular}{lcccc}
\multicolumn{1}{c}{ Center } & Population & \multicolumn{2}{c}{ Location } \\
\cline { 2 - 4 } & & & \\
Portland SMSA & $1,200,000$ & $60 \mathrm{~km}$ & SSE \\
Longview & 31,000 & $12 \mathrm{~km}$ & $\mathrm{NNW}$ \\
Astoria & 13,000 & $72 \mathrm{~km}$ & WNW \\
Forest Grove & 12,000 & $58 \mathrm{~km}$ & SSW \\
Centralia & 11,000 & $75 \mathrm{~km}$ & $\mathrm{~N}$
\end{tabular}

SITE SPECIFIC DATA - AIRBORNE PATHWAYS

Average Annual State Production

of Crops and Anjmal Products

In 80-km Radius Circle

Regional Productivity Factor:

Animal Grazing Factor:
Veg: $6.4 \mathrm{E} 7 \mathrm{kilogram}$

Milk: 3.7E7 Titer

Meat: $2.6 \mathrm{E} 7$ kilogram

0.9

0.75

Meteorology Period of Record: 1 SEP 71 - 31. AUG 74 Recovery: 90\%

SITE SPECIFIC DATA - WATERBORNE PATHWAYS via COLUMBIA RIVER

Average River Flow

at Site: $2.3 \mathrm{E} 5 \mathrm{ft}^{3} / \mathrm{s}$

Drinking Water:

Exposed Population: $530(\mathrm{a})$

Dilution Factor: 1

Fish:

Edible Harvest: 1. DE6 $\mathrm{kg} / \mathrm{yr}$

Dilution Factor: 1

(a) Population of Rainier divided by 4 , since residents only there for $25 \%$ of the year. 


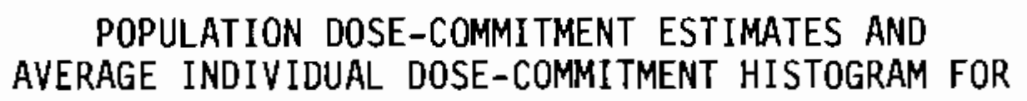

TROJAN

Dose Commitments (person-rem) from Liquid Pathways

\begin{tabular}{|c|c|c|c|c|c|}
\hline & Total Body & GI-LII & Thyroid & Bone & Liver \\
\hline $\begin{array}{l}\text { Infant } \\
\text { Child } \\
\text { Teen } \\
\text { Adult }\end{array}$ & $\begin{array}{l}1.9 \mathrm{E}-06 \\
4.3 \mathrm{E}-03 \\
6.2 \mathrm{E}-03 \\
6.1 \mathrm{E}-02\end{array}$ & $\begin{array}{l}6.0 \mathrm{E}-07 \\
1.5 \mathrm{E}-02 \\
3.2 \mathrm{E}-02 \\
2.7 \mathrm{E}-01\end{array}$ & $\begin{array}{l}3.4 \mathrm{E}-06 \\
5.0 \mathrm{E}-04 \\
3.5 \mathrm{E}-04 \\
2.3 \mathrm{E}-03\end{array}$ & $\begin{array}{l}7.0 \mathrm{E}-06 \\
2.0 \mathrm{E}-02 \\
1.4 \mathrm{E}-02 \\
8.8 \mathrm{E}-02\end{array}$ & $\begin{array}{l}6.9 \mathrm{E}-07 \\
1.4 \mathrm{E}-02 \\
1.2 \mathrm{E}-02 \\
6.9 \mathrm{E}-02\end{array}$ \\
\hline TOTAL & $7.2 \mathrm{E}-02$ & $3.2 \mathrm{E}-01$ & 3. $2 \mathrm{E}-03$ & 1.2E-01 & $9.4 \mathrm{E}-02$ \\
\hline
\end{tabular}

Dose Commitments (person-rem) from Airborne Pathways

Total Body GI-LII Thyroid Bone Liver Lung

$\begin{array}{lllllll}\text { Infant } & 9.7 \mathrm{E}-04 & 9.6 \mathrm{E}-04 & 2.2 \mathrm{E}-03 & 6.8 \mathrm{E}-04 & 9.6 \mathrm{E}-04 & 1.0 \mathrm{E}-03 \\ \text { Child } & 1.8 \mathrm{E}-02 & 1.6 \mathrm{E}-02 & 2.8 \mathrm{E}-02 & 1.7 \mathrm{E}-02 & 1.6 \mathrm{E}-02 & 1.7 \mathrm{E}-02 \\ \text { Teen } & 1.2 \mathrm{E}-02 & 1.1 \mathrm{E}-02 & 1.7 \mathrm{E}-02 & 1.0 \mathrm{E}-02 & 1.1 \mathrm{E}-02 & 1.3 \mathrm{E}-02 \\ \text { Adult } & 7.0 \mathrm{E}-02 & 6.7 \mathrm{E}-02 & 9.1 \mathrm{E}-02 & 5.5 \mathrm{E}-02 & 6.6 \mathrm{E}-02 & 7.1 \mathrm{E}-02 \\ \text { TOTAL } & 1.0 \mathrm{E}-01 & 9.5 \mathrm{E}-02 & 1.4 \mathrm{E}-01 & 8.2 \mathrm{E}-02 & 9.4 \mathrm{E}-02 & 1.0 \mathrm{E}-01\end{array}$

Production/Consumption factors:

Produce: <1 Milk: <1 Meat: <1

FRACTIDN OF POPULATION RECEIVING AN AVERAGE INDIVIDUAL TOTAL-BOOY DOSE COMMITMENT FROM AIRBORNE PATHWAYS

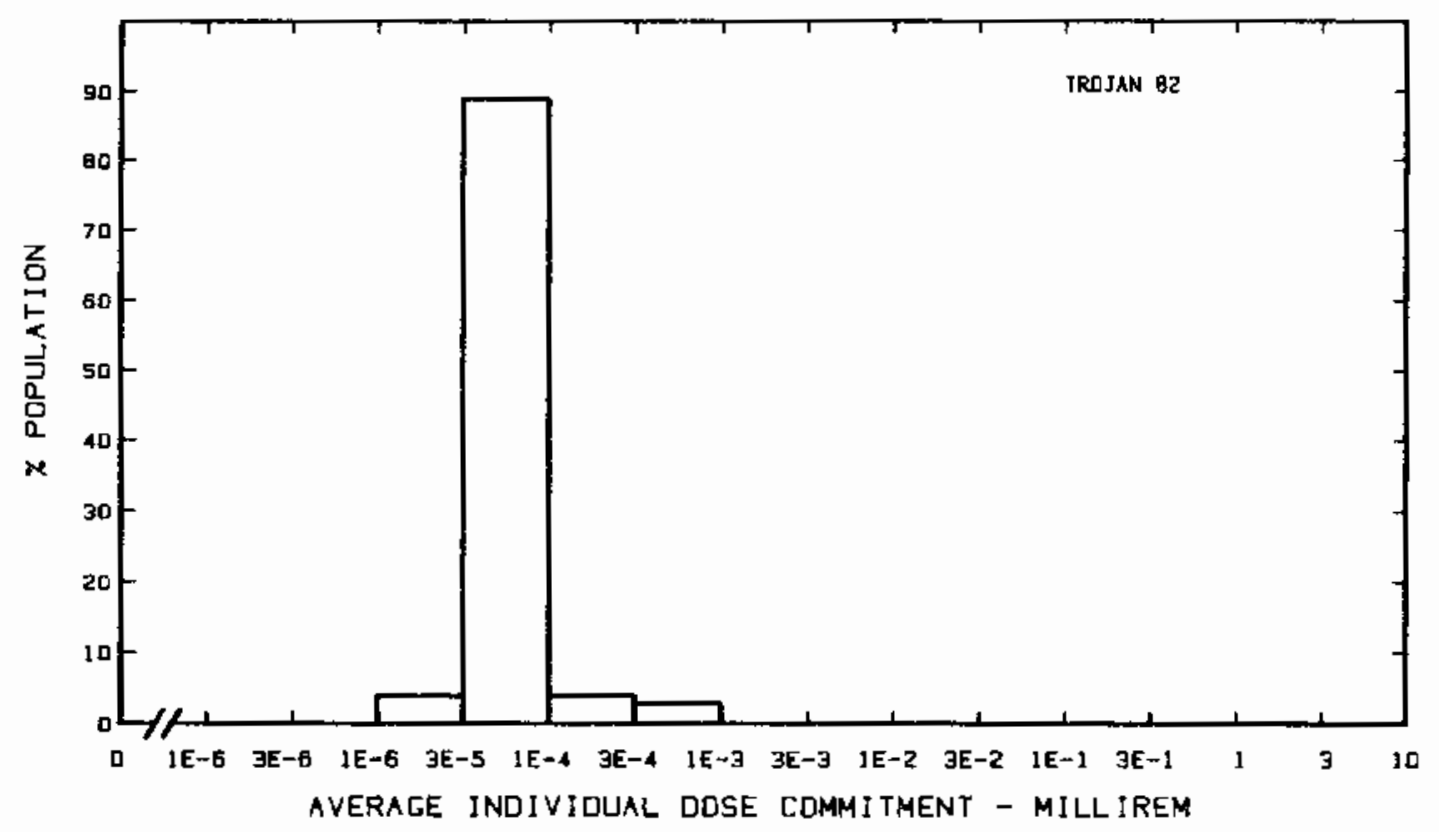


Site: TURKEY POINT

DADE COUNTY, FLORIDA

Location: N $25.4350^{\circ}$ W $80.3314^{\circ}$

POPULATION DATA

Total Population Within 2-to-80-km Region: 2.4E6

Major Metropolitan Centers Within Region:

Center Population Location

Miami SMSA

$1,600,000$

Fort Lauderdale-Hollywood SMSA $1,000,000$

Homestead

21,000

Upper Keys Division

15,000

$41 \mathrm{~km} \quad$ NNE

$79 \mathrm{~km} \quad \mathrm{NNE}$

$16 \mathrm{~km} \quad W$

$42 \mathrm{~km} \mathrm{~S}$

SITE SPECIFIC DATA - AIRBORNE PATHWAYS

Average Annual State Production

of Crops and Animal Products

In $8 D-k m$ Radius Circle

Veg: $2.8 E 7$ kilogram

Mi 1k: $1.1 \mathrm{E} 8$ 1iter

Regional Productivity Factor:

Animal Grazing Factor:

Meat: $7.2 \mathrm{E} 7 \mathrm{kilogram}$

Meteorology Period of Record: 1 JAN 73 - 31 DEC 73 Recovery: 98\%

SITE SPECIFIC DATA - WATERBORNE PATHWAYS via BISCAYNE BAY

Average Dilution Flow

from Plant: $3,100 \mathrm{ft}^{3} / \mathrm{s}$

Fish:

Edible Harvest: (a)

Dilution Factor: 0.001

Invertebrates:

Edible Harvest:

(a)

Dilution Factor: D.002

(a) No catch data given in DES (1972), so generic consumption rates used (Table A-1). 


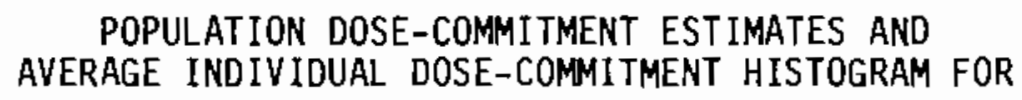

TURKEY POINT 3 AND 4

Dose Commitments (person-rem) from Liquid Pathways

Total Body GI-LII Thyrojd Bone Liver

$\begin{array}{llllll}\text { Infant } & 0.0 \mathrm{E}+00 & 0.0 \mathrm{E}+00 & 0.0 \mathrm{E}+00 & 0.0 \mathrm{E}+00 & 0.0 \mathrm{E}+00 \\ \text { Child } & 6.3 \mathrm{E}-04 & 1.9 \mathrm{E}-03 & 1.6 \mathrm{E}-02 & 5.5 \mathrm{E}-04 & 7.9 \mathrm{E}-04 \\ \text { Teen } & 5.3 \mathrm{E}-04 & 4.0 \mathrm{E}-03 & 1.1 \mathrm{E}-02 & 3.3 \mathrm{E}-04 & 6.8 \mathrm{E}-04 \\ \text { Aduit } & 3.9 \mathrm{E}-03 & 3.5 \mathrm{E}-02 & 7.3 \mathrm{E}-02 & 2.0 \mathrm{E}-03 & 4.1 \mathrm{E}-03 \\ \text { TOTAL } & 5.1 \mathrm{E}-03 & 4.1 \mathrm{E}-02 & 1.0 \mathrm{E}-01 & 2.8 \mathrm{E}-03 & 5.6 \mathrm{E}-03\end{array}$

Dose Commitments (person-rem) from Airborne Pathways

Total Body GI-LII Thyroid Bone Liver lung

$\begin{array}{lllllll}\text { Infant } & 4.4 \mathrm{E}-03 & 4.3 \mathrm{E}-03 & 1.2 \mathrm{E}-01 & 4.6 \mathrm{E}-03 & 4.6 \mathrm{E}-03 & 4.6 \mathrm{E}-03 \\ \text { Child } & 4.8 \mathrm{E}-02 & 4.7 \mathrm{E}-02 & 6.7 \mathrm{E}-01 & 4.9 \mathrm{E}-02 & 4.9 \mathrm{E}-02 & 5.3 \mathrm{E}-02 \\ \text { Teen } & 3.5 \mathrm{E}-02 & 3.5 \mathrm{E}-02 & 2.8 \mathrm{E}-01 & 3.5 \mathrm{E}-02 & 3.5 \mathrm{E}-02 & 4.1 \mathrm{E}-02 \\ \text { AduTt } & 2.1 \mathrm{E}-01 & 2.1 \mathrm{E}-01 & 1.0 \mathrm{E}-00 & 2.1 \mathrm{E}-01 & 2.1 \mathrm{E}-01 & 2.3 \mathrm{E}-01 \\ \text { TOTAL } & 3.0 \mathrm{E}-01 & 3.0 \mathrm{E}-01 & 2.1 \mathrm{E}-00 & 3.0 \mathrm{E}-01 & 3.0 \mathrm{E}-01 & 3.3 \mathrm{E}-01\end{array}$

Production/Consumption factors:

Produce: $<1 \quad$ Mi1k: <1 Meat: <1

FRACTION DF POPULATION RECEIVING AN AVERAGE INDIVIOUAL TOTAL-BODY OOSE COMMITMENT FROM AIRBORNE PATHWAYS

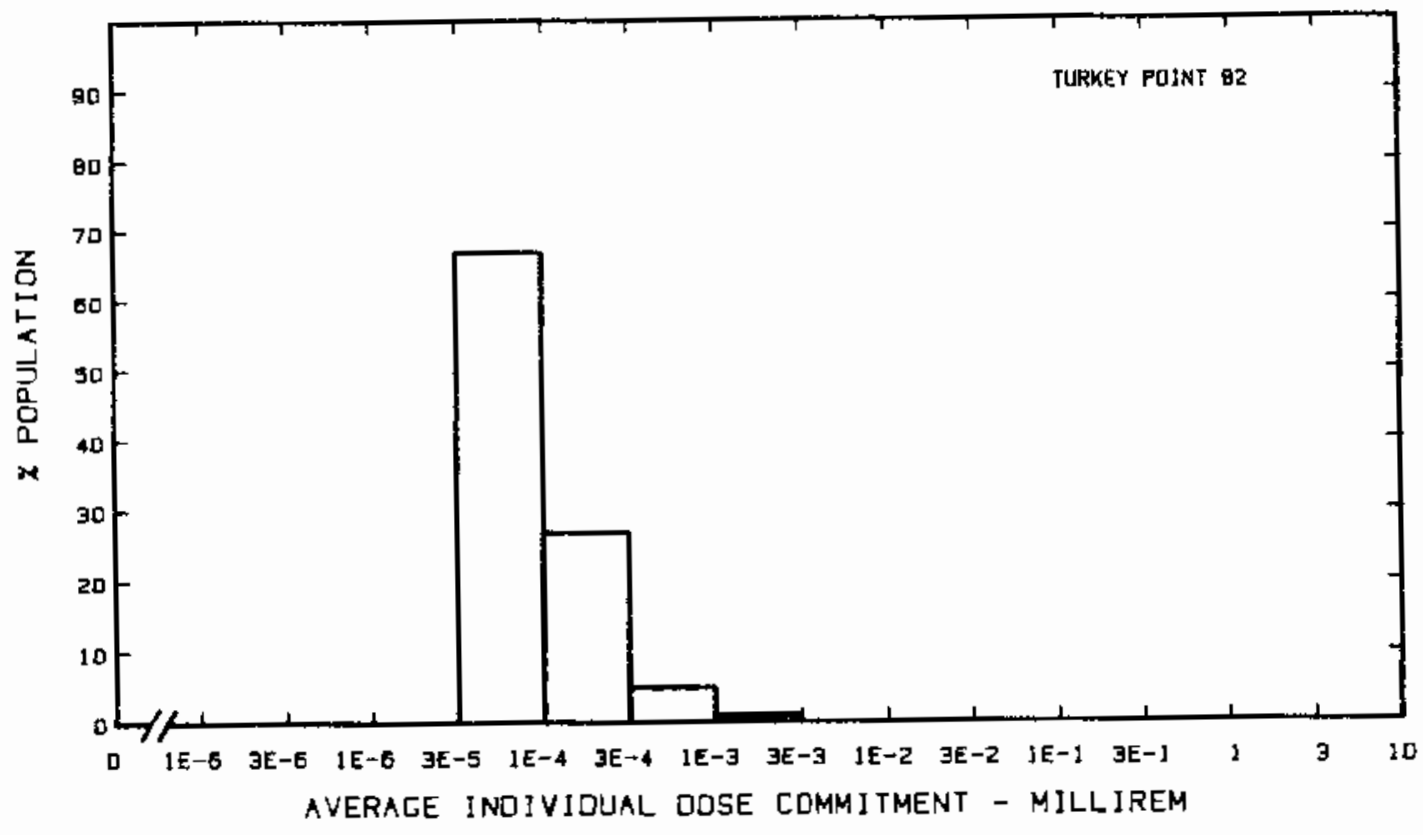


Site: VERMONT YANKEE

VERNON, VERMONT

Location:

$\mathrm{N} \quad 42.7803^{\circ}$

W $72.5158^{\circ}$

POPULATION OATA

Total Population Within 2-to-80-km Region: 1.3E6

Major Metropolitan Centers Within Region:

Center Population Location

Springfield-Chicopee-Holyoke SMSA $\quad 530,000 \quad 70 \mathrm{~km} \quad \mathrm{~S}$

Worcester SMSA (1/2) $\quad 140,000 \quad 80 \mathrm{~km}$ SE

Pittsfield

$91,000 \quad 71 \mathrm{~km} \quad \mathrm{SW}$

Fitchburg

$40,000 \quad 63 \mathrm{~km} \quad$ ESE

Leominster

35,000

Keene

21,000

$68 \mathrm{~km} \quad \mathrm{ESE}$

$26 \mathrm{~km} \quad \mathrm{NW}$

SITE SPECIFIC DATA - AIRBORNE PATHWAYS

Average Annual State Production

Of Crops and Animal Products

In 80-km Radius Circle

Veg: 4.4E6 kilogram

Milk: $7.3 \mathrm{E} 8$ liter

Regional Productivity Factor:

Animal Grazing Factor:

Meat: 2.7E7 kilogram

1

0.4

Meteorology Period of Record: 1 APR 75 - 3.. MAR 76 Recovery: 97\%

SITE SPECIFIC DATA - WATERBORNE PATHWAYS via CONNECTICUT RIVER at VERNON POND

Average River Flow

at Site: $10,000 \mathrm{ft}^{3} / \mathrm{s}$

Drinking Water:

Exposed Population: None

Fish:

Edible Harvest: (a)

Dilution Factor:

(a) No radionuclides released in liquid effluents reported (Tichler and Norden 1985). 


\section{POPULATION DOSE-COMMITMENT ESTIMATES AND \\ AVERAGE INDIVIDUAL DOSE-COMMITMENT HISTOGRAM FOR \\ VERMONT YANKEE}

Dose Commitments (person-rem) from Liquid Pathways

Total Body GI-LII $\underline{\text { Thyroid }}$ Bone

Infant

Child

Teen

(No Liquid Pathway Doses)

Adult

TOTAL

Dose Commitments (person-rem) from Airborne Pathways

Total Body GI-LII Thyroid Bone Liver Lung

Infant

8. $3 \mathrm{E}-04$

8. $3 \mathrm{E}-04$

9. 3E-D4

4. $9 \mathrm{E}-04$

8.7E-04

8. $9 \mathrm{E}-04$

Child

9. $5 \mathrm{E}-03$

9. $5 \mathrm{E}-\mathrm{D} 3$

1. 0E-02

5. $4 \mathrm{E}-03$

$9.8 \mathrm{E}-03$

1. $1 \mathrm{E}-02$

Teen

6.7E-03

$6.7 \mathrm{E}-03$

7. $1 \mathrm{E}-03$

$3.8 \mathrm{E}-03$

$6.8 \mathrm{E}-03$

7. $7 \mathrm{E}-03$

Adult

3.8E-02

3. $8 \mathrm{E}-02$

4. $0 \mathrm{E}-02$

2. $3 \mathrm{E}-02$

3. $9 \mathrm{E}-02$

4. 2E-02

TOTAL

$5.6 \mathrm{E}-02$

5. $5 \mathrm{E}-02$

5. 8E-02

3. $3 \mathrm{E}-02$

$5.6 \mathrm{E}-02$

6. $1 \mathrm{E}-02$

Production/Consumption factors:

Produce: $<1$

Milk: 4.2

Meat: <1

FRACTION OF POPULATION RECEIVING AN AVERAGE INDIVIDUAL TOTAL-BOOY OOSE COMMITMENT FROM AIRBORNE PATHWAYS

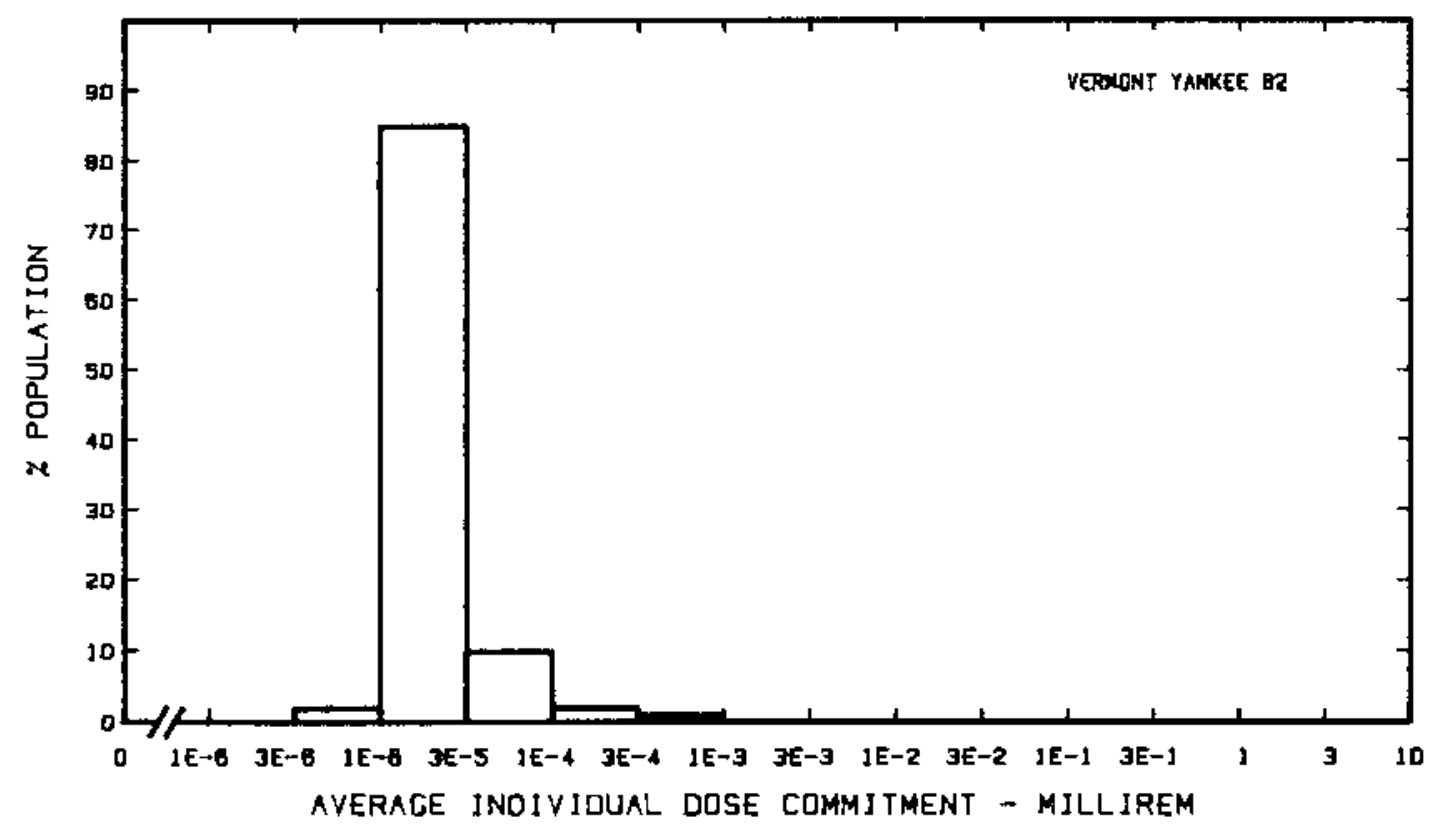


Site: YANKEE ROWE

ROWE, MASSACHUSETTS

Location: $\quad$ N $42.7281^{\circ}$ W $72.9289^{\circ}$

POPULATION DATA

Total Population Within 2-to-80-km Region: $1.6 E 6$

Major Metropolitan Centers Within Region:

Center

Population Location

Springfield-Chicopee-Holyoke SMSA $\quad 530,000$

Albany

Pittsfield SMSA

100,000

Troy

91,000

Amherst

57,000

18,000

$74 \mathrm{~km} \quad$ SSE

$68 \mathrm{~km} \quad \mathrm{~W}$

$41 \mathrm{~km} \quad \mathrm{SW}$

$62 \mathrm{~km} \quad \mathrm{~W}$

$51 \mathrm{~km} \quad \mathrm{SE}$

SITE SPECIFIC DATA - AIRBORNE PATHWAYS

Average Annual State Production

of Crops and Animal Products

In $80-\mathrm{km}$ Radius Circle

Regional Productivity Factor:

Animal Grazing Factor:

Meteorology Period of Record: 1 OCT 71 - 30 SEP 72 Recovery: 94\%
Veg: $2.0 \mathrm{E} 7$ kilogram

Mi ik: 2.6E8 liter

Meat: 1.6E7 kilogram

1

0.5

SITE SPECIFIC DATA - WATERBORNE PATHWAYS via DEERFIELO RIVER

Average River Flow at Site: $570 \mathrm{ft}^{3} / \mathrm{s}$

Drinking Water:

Exposed Population: None

Fish:

Edible Harvest: (a)

Dilution Factor: 0.025 (b)

(a) No catch data available, so generic consumption rates used (Table A-1).

(b) Ten percent of population obtain $25 \%$ of their fish from river. 
POPULATION DOSE-COMMITMENT ESTIMATES AND AVERAGE INDIVIDUAL OOSE-COMMITMENT HISTOGRAM FOR

\section{YANKEE ROWE}

Dose Commitments (person-rem) from Liquid Pathways

\begin{tabular}{llllll} 
& Total Body & GI-LII & Thyroid & Bone & Liver \\
\cline { 2 - 3 } Infant & $0.0 \mathrm{E}+00$ & $0.0 \mathrm{E}+00$ & $0.0 \mathrm{E}+00$ & $0.0 \mathrm{E}+00$ & $0.0 \mathrm{E}+00$ \\
Child & $6.6 \mathrm{E}-03$ & $2.1 \mathrm{E}-03$ & $5.3 \mathrm{E}-03$ & $2.9 \mathrm{E}-02$ & $3.0 \mathrm{E}-02$ \\
Teen & $1.1 \mathrm{E}-02$ & $2.2 \mathrm{E}-03$ & $3.9 \mathrm{E}-03$ & $1.7 \mathrm{E}-02$ & $2.5 \mathrm{E}-02$ \\
Adult & $1.1 \mathrm{E}-01$ & $1.7 \mathrm{E}-02$ & $2.6 \mathrm{E}-02$ & $1.0 \mathrm{E}-01$ & $1.5 \mathrm{E}-01$ \\
TOTAL & $1.3 \mathrm{E}-01$ & $2.1 \mathrm{E}-02$ & $3.6 \mathrm{E}-02$ & $1.5 \mathrm{E}-01$ & $2.0 \mathrm{E}-01$
\end{tabular}

Dose Commitments (person-rem) from Airborne Pathways Total Body GI-LII Thyroid Bone Liver Lung

$\begin{array}{lllllll}\text { Infant } & 3.2 \mathrm{E}-03 & 3.2 \mathrm{E}-03 & 3.5 \mathrm{E}-03 & 1.4 \mathrm{E}-02 & 3.2 \mathrm{E}-03 & 3.2 \mathrm{E}-03 \\ \text { Child } & 2.2 \mathrm{E}-02 & 2.2 \mathrm{E}-02 & 2.3 \mathrm{E}-02 & 1.0 \mathrm{E}-01 & 2.2 \mathrm{E}-02 & 2.2 \mathrm{E}-02 \\ \text { Teen } & 7.4 \mathrm{E}-03 & 7.4 \mathrm{E}-03 & 8.0 \mathrm{E}-03 & 3.1 \mathrm{E}-02 & 7.5 \mathrm{E}-03 & 7.5 \mathrm{E}-03 \\ \text { Adult } & 2.6 \mathrm{E}-02 & 2.6 \mathrm{E}-02 & 2.8 \mathrm{E}-02 & 9.1 \mathrm{E}-02 & 2.6 \mathrm{E}-02 & 2.6 \mathrm{E}-02 \\ \text { TOTAL } & 5.8 \mathrm{E}-02 & 5.8 \mathrm{E}-02 & 6.2 \mathrm{E}-02 & 2.4 \mathrm{E}-01 & 5.8 \mathrm{E}-02 & 5.8 \mathrm{E}-02\end{array}$

Production/Consumption factors:
Produce: $<1$
Milk: 1.2
Meat: $<1$

FRACTIDN OF PQPLLATION RECEIVING AN AVERAGE INDIVIDUAL TOTAL-BDOY DOSE CDMMITMENT FROM AIRBDRNE PATHWAYS

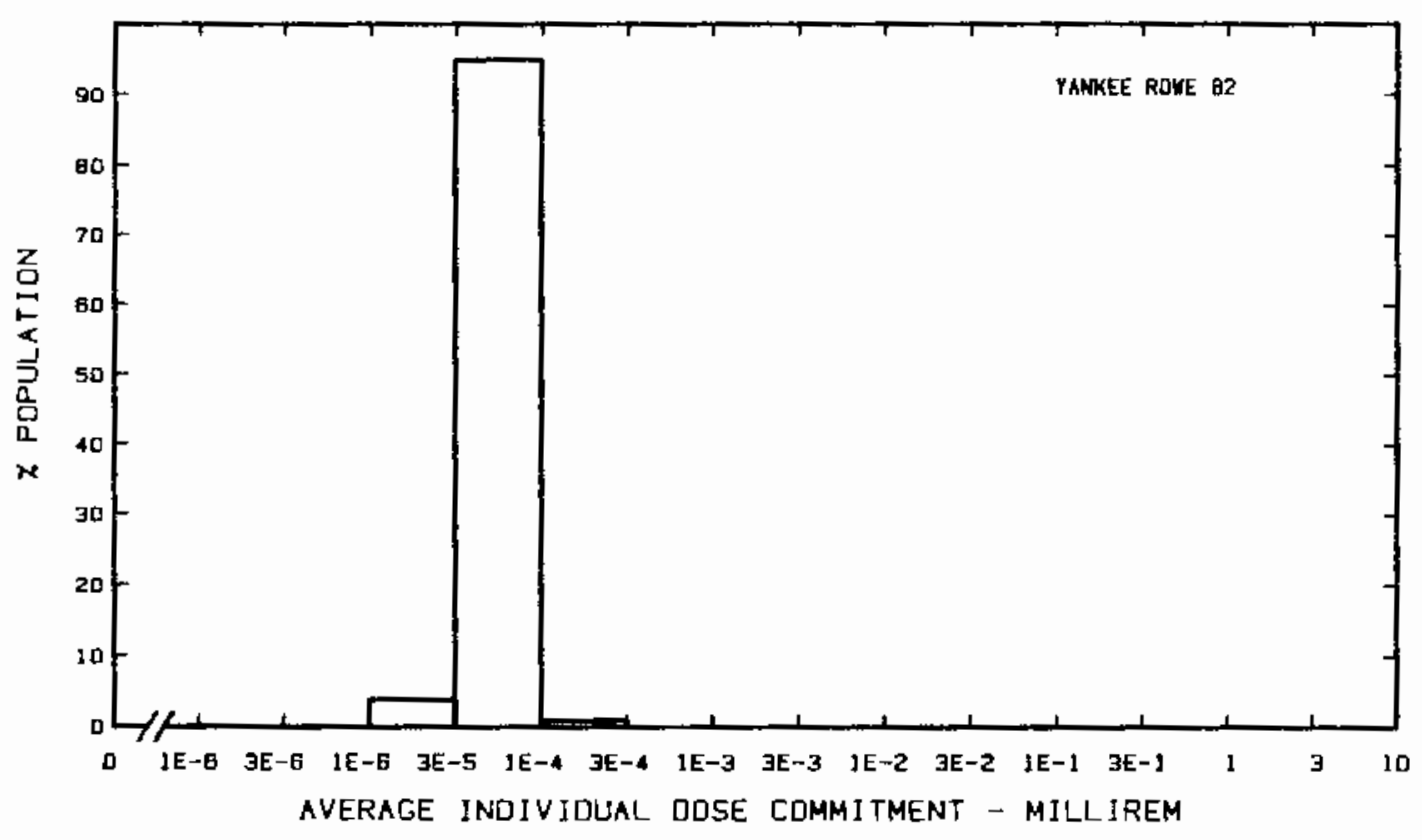


Site: ZION

ZION, ILLINOIS

Location: $\quad$ N $42.4456^{\circ} \quad$ W $87.8022^{\circ}$

POPULATION DATA

Total Population Within 2-to-80-kn Region: 7.1E6

Major Metropolitan Centers Within Region:

\begin{tabular}{lrrrr}
\multicolumn{1}{c}{ Center } & Population & \multicolumn{2}{c}{ location } \\
Chicago SMSA & $(2 / 3)$ & $4,700,000$ & & \\
Milwaukee SMSA & $1,400,000$ & $66 \mathrm{~km}$ & $\mathrm{~S}$ \\
Racine SMSA & 170,000 & $65 \mathrm{~km}$ & $\mathrm{~N}$ \\
Kenosha SMSA & 120,000 & $30 \mathrm{~km}$ & $\mathrm{~N}$ \\
Waukesha & 50,000 & $14 \mathrm{~km}$ & $\mathrm{~N}$ \\
Wand & & $71 \mathrm{~km}$ & NNW
\end{tabular}

SITE SPECIFIC DATA - AIRBORNE PATHWAYS

Average Annual State Production

Of Crops and Anjmal Products

In 80-km Radius Circle

Regional Productivity Factor:

Animal Grazing Factor:
Veg: $1.1 \mathrm{E} 8 \mathrm{ki}$ logram

Milk: $1.8 \mathrm{E} 8$ liter

Meat: $1.9 E 8$ kilogram

0.5

0.5

Meteorology Period of Record: 1 JANN 74 - 31 DEC 75 Recovery: $88 \%$

SITE SPECIFIC DATA - WATERBORNE PATHWAYS via LAKE MICHIGAN

Average Dilution Flow

from Plant: $7,600 \mathrm{ft}^{3} / \mathrm{s}$

Drinking Water:

Exposed Population: 6,809,000

Dilution Factor: 0.037

Fish:

Edible Harvest: $5.0 \mathrm{E} 6 \mathrm{(kg} / \mathrm{yr}$
Dilution Factor: 0.01

(a) Drinking-water dilution factor estimated by averaging dilution factors derived from FES (1972) suitably weighted for population.

(b) Dilution factor derived from FES (1972). 


\section{POPULATION DOSE-CONITMENT ESTIMATES AND AVERAGE INDIVIDUAL DOSE-COMMITMENT HISTOGRAY FOR}

\section{ZION 1 AND 2}

Dose Comitments (person-rem) from Liquid Pathways

Total Body GI-LII Thyroid Bone Liver

$\begin{array}{llllll}\text { Infant } & 5.6 \mathrm{E}-03 & 5.0 \mathrm{E}-03 & 1.8 \mathrm{E}-03 & 1.1 \mathrm{E}-01 & 7.9 \mathrm{E}-03 \\ \text { Child } & 6.6 \mathrm{E}-02 & 9.9 \mathrm{E}-02 & 1.2 \mathrm{E}-02 & 1.0 \mathrm{E}+00 & 1.1 \mathrm{E}-01 \\ \text { Teen } & 3.4 \mathrm{E}-02 & 9.3 \mathrm{E}-02 & 3.8 \mathrm{E}-03 & 2.6 \mathrm{E}-01 & 5.5 \mathrm{E}-02 \\ \text { Adult } & 3.1 \mathrm{E}-01 & 8.3 \mathrm{E}-01 & 2.7 \mathrm{E}-02 & 1.6 \mathrm{E}-00 & 3.4 \mathrm{E}-01 \\ \text { TOTAL } & 4.2 \mathrm{E}-01 & 1.0 \mathrm{E}+00 & 4.5 \mathrm{E}-02 & 3.0 \mathrm{E}+00 & 5.1 \mathrm{E}-01\end{array}$

Dose Commitments (person-rem) from Airborne Pathways Total Body GI-LII Thyroid Bone Liver Lung

$\begin{array}{lllllll}\text { Infant } & 3.1 \mathrm{E}-02 & 3.0 \mathrm{E}-02 & 3.3 \mathrm{E}-02 & 3.3 \mathrm{E}-02 & 3.5 \mathrm{E}-02 & 3.2 \mathrm{E}-02 \\ \text { Child } & 3.5 \mathrm{E}-01 & 3.4 \mathrm{E}-01 & 3.6 \mathrm{E}-01 & 3.7 \mathrm{E}-01 & 3.9 \mathrm{E}-01 & 3.6 \mathrm{E}-01 \\ \text { Teen } & 2.6 \mathrm{E}-01 & 2.5 \mathrm{E}-01 & 2.6 \mathrm{E}-01 & 2.6 \mathrm{E}-01 & 2.7 \mathrm{E}-01 & 2.8 \mathrm{E}-01 \\ \text { Adu1t } & 1.6 \mathrm{E}+00 & 1.5 \mathrm{E}+00 & 1.5 \mathrm{E}+00 & 1.5 \mathrm{E}+00 & 1.6 \mathrm{E}+00 & 1.6 \mathrm{E}+00 \\ \text { TOTAL } & 2.2 \mathrm{E}+00 & 2.1 \mathrm{E}+00 & 2.2 \mathrm{E}+00 & 2.2 \mathrm{E}+0 \mathrm{D} & 2.3 \mathrm{E}+00 & 2.3 \mathrm{E}+00\end{array}$

Production/Consumption factors:

Produce: <1 Milk: <1 Meat: <1

FRACTION OF POPULATION RECEIVING AN AVERAGE INDIVIDUAL TOTAL-BODY DOSE COMMITMENT FRDM AIRBORNE PATHWAYS

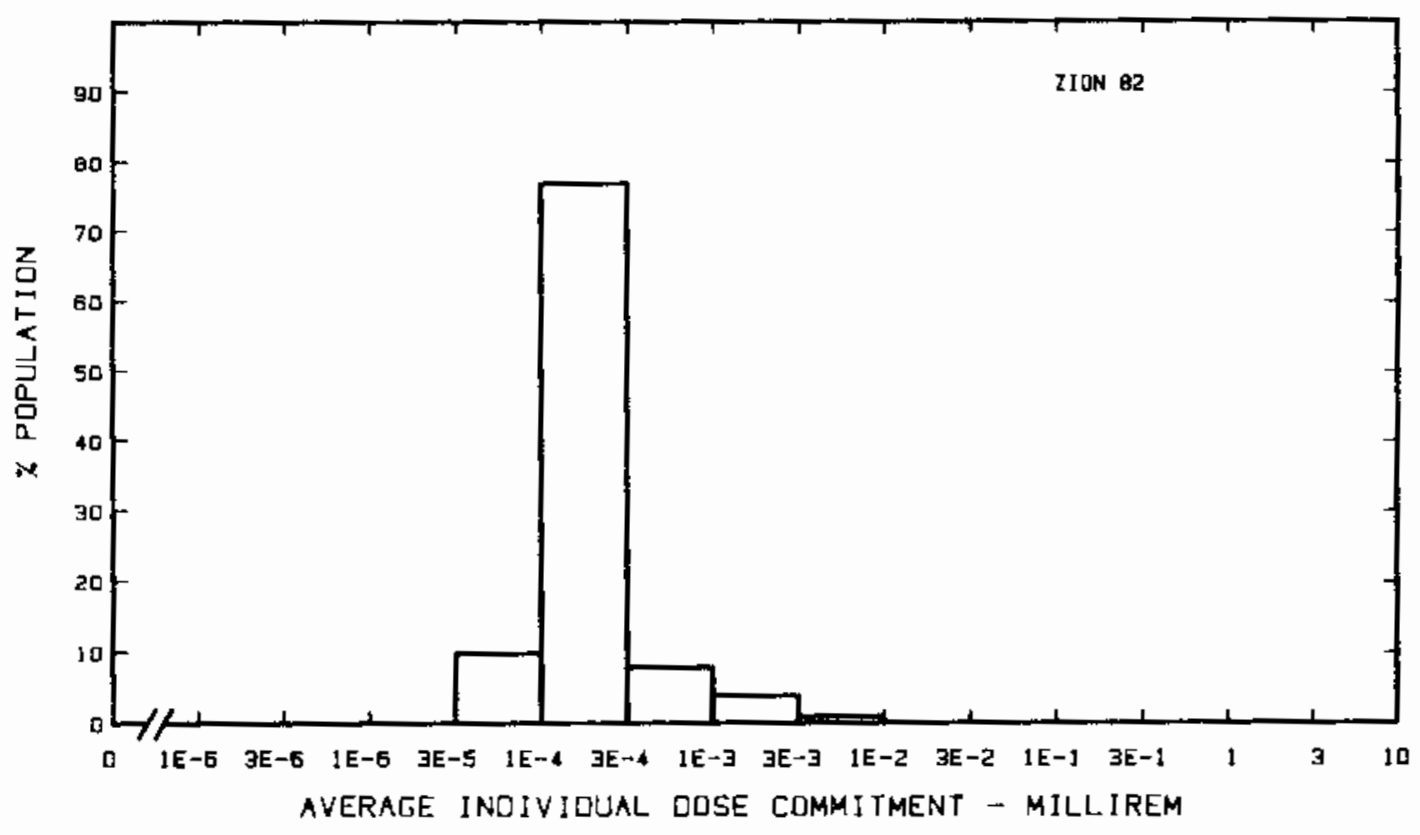





\section{REFERENCES}

Final Environmental Statement Concerning Proposed Rule-Making Action: Numerical Guides for Design objectives and Limiting Conditions for Operation to Meet the Criterion "As Low As Practicable" for Radioactive Material in Light-Water-Cooled Nuclear Power Reactor Effiuents. 1973. WASH-1258, Vol. 1, Directorate of Regulatory Standards, U.S. Atomic Energy Commission, Washington, D.C.

Tichler, J., and K. Norden. 1985. Radioactive Materials Released from Nuclear Power Plants, Annual Report 1982. NUREG/CR-2907, BNLNUREG-51581, Vo1. 3, U.S. Nuclear Regulatory Comission, Washington, D.C. *

*Available for purchase from the NRC/GPO Sales Program, U.S. Nuclear Regulatory Commission, Washington, DC 20555, and the National Technical Information Service, Springfield, VA 22161. 

APPENDIX

MOOELS AND GENERIC PARAMETERS

The calculational models used were primarily those given in the Nuclear Regulatory Commission's Regulatory Guide 1.109 (NRC 1977). Computer programs were written to use these models to generate population dose commitments for four age groups. The percentages of the population comprising the four age groups were $1.44 \%$, infant; $16.0 \%$, child; $11.7 \%$, teenager; and 70.9\%, aduit (Population Estimates and Projections 1975). Where possible, the site-dependent parameters were taken from the environmental statements issued for each reactor (Table 3). The generic parameters used for this study such as consumption rates, occupancy factors and holdup times are given in Tables $A-1$ and $A-2$ below. It should be noted that generic consumption rates for aquatic foods and inhalation rates are taken from Regulatory Guide 1.109 (NRC 1977). Bioaccumulation factors and terrestrial food transfer factors were taken from Regulatory Guide 1.109 (NRC 1977). Dose commitment factors for the four age groups were taken from Hoenes and Soldat (1977).

TABLE A-1. Generic Consumption Rates and 0ccupancy Factors Used, for the Study of Average Members of the Population

\begin{tabular}{|c|c|c|c|c|}
\hline Pathway & Infant & Child & Teenager & Adult \\
\hline $\begin{array}{l}\text { Fruits, vegetables and grain } \\
(\mathrm{kg} / \mathrm{yr})\end{array}$ & 0 & 200 & 240 & 190 \\
\hline Milk (L/yr) & 170 & 170 & 200 & 110 \\
\hline Meat and poultry $(\mathrm{kg} / \mathrm{yr})$ & 0 & 37 & $5 \mathrm{~g}$ & 95 \\
\hline Fish $(\mathrm{kg} / \mathrm{yr})^{(\mathrm{b})^{2}}$ & 0 & 2.2 & 5.2 & 6.9 \\
\hline Invertebrates $(\mathrm{kg} / \mathrm{yr})$ & $0(a)$ & 0.33 & 0.75 & 1.0 \\
\hline Drinking water (L/yr) & $170^{(c)}$ & 260 & 260 & 370 \\
\hline Inhalation $\left(\mathrm{m}^{3} / \mathrm{yr}\right)$ & $1400^{(d)}$ & 3700 & 8000 & 8000 \\
\hline $\begin{array}{l}\text { Air submersion and ground } \\
\text { irradiation occupancy } \\
\text { factor }\end{array}$ & 0.5 & 0.5 & 0.5 & 0.5 \\
\hline
\end{tabular}

(a) Regulatory Guide 1.109 (NRC 1977).

(b) Both fresh and salt water.

(c) Assumed to be equal to milk consumption.

(d) Same as for maximum individual. 
TABLE A-2. Holdup Times Between (Harvest and
Consumption of Foods

Food

Fruifs, grains and vegetables

Milk (b)

Meat

Aquatic foods (fish and invertebrates)

Drinking Water
Holdup Time (days)

14

4

20

7

1

(a) Regulatory Guide 1.109 (NRC 1977)

(b) Value given is time after milking or slaughter. For the portion of the time anirials were fed stored feed, an additional 90 days was added to the holdup time.

SOURCE TERMS

The doses were estimated using the measured releasgs as reported by the site operators for 1981 (Tichler and Norden 1985). ${ }^{\text {a) The releases }}$ include all radionuclides specified by the NRC to be measured and reported by the operators of all commercial nuclear power plants. Radionuclides given as a combination of parent-daughter isotopes such as $\mathrm{Y} / \mathrm{Sr}-90, \mathrm{Zr} / \mathrm{Nb}-95, \mathrm{Ba} / \mathrm{La}-140, \mathrm{I} / \mathrm{Xe}-133$ and $\mathrm{Pr} / \mathrm{Ce}-144$ were divided eventy between the parent and daughter.

The radionuclides used in this study, along with their half-lives, are given in Table A-3. Note that the "+D" after some of the nuclides indicates that the decay energy of the daughter is included with the parent. Thus, whenever a parent nuclide release is specified, the result of the dose calculation is as though an additional equilibrium amount of the daughter nuclide is specified. The daughter nuclide itself is included separately if it can be released independently of the parent and/or if it has a relatively long half-life.

(a) Very shortlived isotopes such as $\mathrm{Kr}-90,91,93,94, \mathrm{Xe}-139,140,141$, 143 and Rb-88M; those not 1ikely to be produced; and those that were daughters whose decay energies were accounted for in the dose factor for the parent were not included in the dose. 
IABLE A-3. Radionuclides Considered in This Study

\begin{tabular}{|c|c|c|c|c|c|}
\hline No. & Nuclide & $\begin{array}{c}\text { Decay Constant } \\
(1 / \mathrm{sec}) \\
\end{array}$ & No. & Nuclide & $\begin{array}{c}\text { Decay Constant } \\
(1 / \mathrm{sec})\end{array}$ \\
\hline 1 & $\mathrm{H}-3$ & $1.78 \mathrm{E}-09$ & 43 & $N b-97$ & $1.57 \mathrm{E}-04$ \\
\hline 2 & $8 e-10$ & $1.37 \mathrm{E}-14$ & 44 & Mo-99+D & 2. $92 \mathrm{E}-06$ \\
\hline 3 & $C-14$ & $3.83 \mathrm{E}-12$ & 45 & Tc-99M & $3.19 \mathrm{E}-05$ \\
\hline 4 & $N-13$ & 1. $16 \mathrm{E}-03$ & 46 & $R u-103+0$ & $2.02 \mathrm{E}-07$ \\
\hline 5 & $F-18$ & $1.05 \mathrm{E}-04$ & 47 & $R u-106+D$ & $2.17 \mathrm{E}-08$ \\
\hline 6 & $\mathrm{Na}-22$ & $8.44 E-09$ & 48 & $\mathrm{Ag}-110 \mathrm{M}+\mathrm{D}$ & 3. 19E-08 \\
\hline 7 & $\mathrm{Na}-24$ & $1.28 \mathrm{E}-05$ & 49 & $C d-115 M$ & $1.80 \mathrm{E}-07$ \\
\hline 8 & $\mathrm{Ar}-41$ & $1.05 \mathrm{E}-04$ & 50 & $C d-115$ & $3.60 \mathrm{E}-06$ \\
\hline 9 & Sc-46 & $9.58 \mathrm{E}-08$ & 51 & $S n-125+D$ & $8.31 \mathrm{E}-07$ \\
\hline 10 & $\mathrm{Cr}-51$ & $2.89 \mathrm{E}-07$ & 52 & Sb-124 & $1.33 \mathrm{E}-07$ \\
\hline 11 & $M n-54$ & $2.57 \mathrm{E}-08$ & 53 & $S b-125+D$ & $8.06 \mathrm{E}-09$ \\
\hline 12 & $M n-56$ & $7.47 \mathrm{E}-05$ & 54 & $\mathrm{Te}-132+\mathrm{D}$ & $2.47 \mathrm{E}-06$ \\
\hline 13 & $\mathrm{Fe}-55$ & $8.14 \mathrm{E}-09$ & 55 & $\mathrm{Te}-133 \mathrm{M}+\mathrm{D}$ & $2.09 \mathrm{E}-04$ \\
\hline 14 & $\mathrm{Fe}-59$ & $1.80 \mathrm{E}-07$ & 56 & $I-131+D$ & $9.97 \mathrm{E}-07$ \\
\hline 15 & $\mathrm{Co}-57$ & 2. $97 \mathrm{E}-08$ & 57 & I-132 & $8.42 \mathrm{E}-05$ \\
\hline 16 & Co-58 & $1.12 \mathrm{E}-07$ & 58 & $I-133+D$ & $9.25 \mathrm{E}-06$ \\
\hline 17 & Co-60 & $4.17 \mathrm{E}-09$ & 59 & I-134 & $2.20 \mathrm{E}-04$ \\
\hline 18 & $\mathrm{Ni}-57$ & $5.35 \mathrm{E}-06$ & 60 & I $-135+0$ & $2.92 E-05$ \\
\hline 19 & $\mathrm{Ni}-63$ & $2.20 \mathrm{E}-10$ & 61 & $\mathrm{Xe}-131 \mathrm{M}$ & $6.69 \mathrm{E}-07$ \\
\hline 20 & $\mathrm{Ni}-65$ & $7.64 \mathrm{E}-05$ & 62 & $\mathrm{Xe}-133 \mathrm{M}$ & $3.61 \mathrm{E}-06$ \\
\hline 21 & $\mathrm{Cu}-64$ & $1.52 \mathrm{E}-05$ & 63 & $X e-133$ & $1.52 \mathrm{E}-06$ \\
\hline 22 & $\mathrm{Zn}-65$ & 3. $31 \mathrm{E}-08$ & 64 & $X e-135 M$ & $7.56 \mathrm{E}-04$ \\
\hline 23 & $Z n-69 M+0$ & 1.39E-05 & 65 & $x_{e}-135$ & $2.10 \mathrm{E}-05$ \\
\hline 24 & As -76 & $7.32 E=06$ & 66 & Xe-137 & $3.01 \mathrm{E}-03$ \\
\hline 25 & $8 r-82$ & $5.44 \mathrm{E}-06$ & 67 & $\mathrm{Xe}-138+\mathrm{D}$ & $8.14 \mathrm{E}-04$ \\
\hline 26 & $\mathrm{Kr}-83 \mathrm{M}$ & $1.04 E-04$ & 68 & $\mathrm{Cs}-134$ & $1.07 \mathrm{E}-08$ \\
\hline 27 & $\mathrm{Kr}-85 \mathrm{M}$ & 4. $31 \mathrm{IE}-05$ & 69 & Cs -136 & $6.17 \mathrm{E}-07$ \\
\hline 28 & $\mathrm{kr}-85$ & $2.05 \mathrm{E}-09$ & 70 & Cs-137+D & $7.31 \mathrm{E}-10$ \\
\hline 29 & $\mathrm{Kr}-87$ & $1.52 \mathrm{E}-04$ & 71 & Cs -138 & $3.58 \mathrm{E}-04$ \\
\hline 30 & $\mathrm{Kr}-88+\mathrm{D}$ & $6.89 \mathrm{E}-05$ & 72 & $C s-139+D$ & $1.24 \mathrm{E}-03$ \\
\hline 31 & $\mathrm{kr}-89$ & $3.64 \mathrm{E}-03$ & 73 & Ba -139 & 1.39E-04 \\
\hline 32 & $\mathrm{Rb}-88$ & $6.53 \mathrm{E}-04$ & 74 & $\mathrm{Ba}-140+\mathrm{D}$ & $6.28 \mathrm{E}-07$ \\
\hline 33 & $\mathrm{Rb}-89+\mathrm{D}$ & $7.61 \mathrm{E}-04$ & 75 & La-140 & $4.78 E-06$ \\
\hline 34 & $S r-89+D$ & $1.59 E-07$ & 76 & La-141 & $4.97 \mathrm{E}-05$ \\
\hline 35 & Sr-90+D & $7.58 \mathrm{E}-10$ & 77 & $\mathrm{Ce}-141$ & $2.47 \mathrm{E}-07$ \\
\hline 36 & $S r-91+D$ & $2.03 \mathrm{E}-05$ & 78 & Ce-144+D & $2.83 \mathrm{E}-08$ \\
\hline 37 & $S r-92+0$ & $7.11 \mathrm{E}-05$ & 79 & Eu-152 & $1.69 \mathrm{E}-09$ \\
\hline 38 & $Y-90$ & $3.01 \mathrm{E}-06$ & 80 & Eu-154 & $2.55 \mathrm{E}-09$ \\
\hline 39 & $Y-91 M+D$ & 2. $32 \mathrm{E}-04$ & 81 & $W-187$ & $8.06 \mathrm{E}-06$ \\
\hline 40 & $2 r-95+D$ & $1.22 \mathrm{E}-07$ & 82 & Th-232+D & $1.57 \mathrm{E}-18$ \\
\hline 41 & $Z r-97+D$ & $1.14 \mathrm{E}-05$ & 83 & $\mathrm{~Np}-239$ & $3.42 \mathrm{E}-06$ \\
\hline 42 & Nb-95 & $\mathrm{E}-07$ & & & \\
\hline
\end{tabular}




\section{METEOROLOGY}

When more than one set of meteorologica: (joint frequency) data was available for a site, the one which appeared to be the most reliable was used to generate atmospheric transport factors. Factors were calculated for 16 compass points and for ten radii from 2 to $80 \mathrm{~km}$ (see Table A-4) using the NRC computer program XOQDOQ (Sagendorf 1977).

\section{TABLE A-4. Radius Intervals and Midpoints for Airborne Dose Calculations (km)}

$\begin{array}{cc}\text { Interval } & \text { Midpoint } \\ 2-3 & 2.5 \\ 3-4 & 3.5 \\ 4-6 & 5 \\ 6-9 & 7.5 \\ 9-14 & 11.5 \\ 14-20 & 17 \\ 20-30 & 25 \\ 30-40 & 35 \\ 40-60 & 50 \\ 60-80 & 70\end{array}$

The XOQDOQ program generates four sets of atmospheric transport factors:

- average annual atmospheric dilution factors, which are not corrected for cloud depletion or radioactive decay

- dilution factors that are only corrected for decay assuming a 2.26-day half-life

- dilution factors that are corrected for depletion and for decay assuming an 8-day half-life

- relative deposition per unit area.

These factors were used to estimate the dose from airborne releases using methods similar to the NRC GASPAR program (Eckerman et al. 1980). The 
transport factors used this year were the same as those used for the previous estimates. The assumptions used in the calculation of these transport factors were as follows:

- 50-m source height with no correction for plume rise or building wake effects

- semi-infinite cloud model with sector-average, Gaussian-plume dispersion

- no correction for terrain height variation.

Since information about height and locations at each site for the releases given in Tichler and Norden (1985) was unavailable, a single generic height of $50 \mathrm{~m}$ was used at each site for the release point. Because the heights and locations of releases are uncertain, estimates of dose to persons living within $2 \mathrm{~km}$ of the site could be in serious error; only persons living between 2 to $80 \mathrm{~km}$ from the site were included in the dose estimates.

POPULATION

The population distribution within 2 to $80 \mathrm{~km}$ around each site was determined from information supplied by the NRC from an updated reduction of 1980 census data (Sinisgalli 1982). Also the NRC supplied updated estimates of the number of people residing in major metropolitan centers within the $80-\mathrm{km}$ region around each site (Brauner 1982).

\section{FOOD PRODUCTION VERSUS FOOD CONSUMPTION}

The total food production for the region within $80 \mathrm{~km}$ around each site was the product of the NRC state-wide productivity figure for each state and a site productivity factor. At some sites this total production may be more or less than the total consumption; i.e., population times average individual consumption (see Table A-1 for generic consumption rates). When production was more than consumption for a site, it was assumed that all persons in the $2-$ to-80-km region ate contaminated food; when production was less than consumption, it was assumed that dilution would occur because uncontaminated food would be shipped into the area from outside. Thus, the calculated doses for a particular food type were reduced in proportion to the ratio of production divided by consumption (production/consumption < 1 ). 
The dose to persons outside the $80-\mathrm{km}$ linit from food shipped out of the region, in the case of production being greater than consumption, is not included in this report because we are concerned only with the dose within the $80-\mathrm{km}$ radius. These production/consumption factors are given for reference as footnotes to the tables showing airborne dose commitment in the Site Summaries Section.

ORINKING WATER

The population between 2 and $80 \mathrm{~km}$ distant from each plant site exposed to drinking water contaminated with released radionuclides was generally obtained from the environmental statement (ES). for the plant. For all sites located on salt water, it was assumed that no dose was received from drinking water. The consumption rates used for drinking water are given in Table A-1.

The radionuclide concentration in the drinking water consumed by a population downstream from a site was usually estimated assuming $100 \%$ mixing of the plant effluent with the river. For lakes, an overall dilution factor was estimated from dilution factors given in the ES for each population center along the shore (within $80 \mathrm{~km}$ ) that consumes the contaminated lake water. These individual factors were weighted by population and averaged to obtain an effective dilution factor for the total population exposed to contaminated drinking water.

AQUATIC FOOD

Wherever possible, the fish-catch data from the plant ES were used to estimate aquatic food consumption rates for the population living within the region. When these data were not found in the ES or were considered unrealistic, the generic values of Table A-1 were used.

The average radionuclide concentration of the waters in which this food was harvested was estimated assuming an additional dilution over the effluent flow from the reactor. For rivers, it: was assumed that the fish were caught in waters in which the plant effluent was completely diluted. For lakes, an additional factor as given in the ES was used; when none was given in the ES, a generic value of 0.01 was used. For ocean and bay sites, generic values of 0.001 and 0.002 were used for fish and invertebrates, respectively, if the ES yielded no values for these parameters. Invertebrates were not assumed to be caught in sufficient 
quantity at freshwater sites (river and lake) to affect the population dose and therefore were not inciuded in the dose calculation. Any exceptions to these general guidelines are explained in the footnotes to the individual site summaries.

\section{TECHNICAL NOTES}

The calculations leading to the dose estimates contained in this report were generated from recent versions of computer programs originally documented in Baker, et. al (1977). The revised programs were written in BASICA and run on an IBM PC microcomputer operating under the $\mathrm{PC}-\mathrm{DOS}$ operating system. The charts were all made using an HP $7470 \mathrm{~A}$ plotter. 


\section{APPENDIX REFERENCES}

Baker, D. A., J. K. Soldat and E. C. Watson. 1977. Population-Dose Commitments Due to Radioactive Releases from Nuclear Power pTant Sites in 1975. PNL-2439, Pacific Northwest Laboratory, Richland, Washington.*

Brauner, A. 1982. Population Estimates, Nuclear Power Plant Nearby Population Concentrations. U.S. Nuclear Regulatory Commission, Washington, D.C.

Eckerman, K. F., F. J. Congel, A. K. Roecklein and W. J. Pasciak. 1980. Users Guide to GASPAR Code. NUREG-0597, U.S. Nuclear Regulatory Commission, washington, D.C.

Hoenes, G. R., and J. K. Soldat. 1977. Age-Specific Radiation Dose Commitment Factors for a One-Year Chronic Intiake. NUREG-0172, U.S. Nuclear Regulatory Commission, Washington, D.C. *

Population Estimates and Projections, Current Population Reports. 1975. Series P-25, No. 541. U.S. Department of (ommerce, Social and Economic Statistics Administration, Bureau of the Census.

Sagendorf, J. F., and J. T. Goll. 1977. X00D0Q--Program for the Meteorological Evaluation of Routine Eff Tuent Releases at Nulcear Power Stations. NUREG-0324, U.S. Nuclear Regulatory Commission, Washington, D.C. ${ }^{\star \star \hbar}-$

Sinisgal1i, A. 1982. 198D Residential Population Estimates, 0-80 Kilometers for Nuclear Power Plants. 0. 5 . Nuclear Regulatory Commission, Washington, D.C.

Statistical Abstract of the United States: 1980. 101st edition. U.S. Department of Commerce, Bureau of the Census, Washington, D.C.

Tichler, J., and K. Norden. 1985. Radioactive Materials Released from Nuclear Power Plants, Annual Report 1982. NOREG-CR-2907, BNL-NUREG-51581, VOT. 3, U.S. NucTear Regulatory Conmission, Washington, D.C.*

U.S. Nuclear Regulatory Commission. 1977. Calculation of Annual Doses to Man from Routine Releases of Reactor EffTuents for the Purpose of Evaluating Compliance with 10 CFR Part 50, Appendix I, Revision 1. Regula tory Guide 1.109, Washington, D.C.

*Available for purchase from the National Technical Information Service, Springfield, VA 22161.

* Available free upon written request to the Division of Technical Information and Document Control, U.S. Nuclear Regulatory Commission, Washington, DC 20555. 
NUREG/CR-2850

PNL-4221

DISTRIBUTION

Vol. 4

No. of

Copies

OFFSITE

U.S. Nuclear Regulatory Commission

Division of Technical Information and Document Control

7920 Norfolk Avenue

Bethesda, MD 20014

MR Beebe

Office of Resource Management

Nuclear Regulatory Commission

Washington, DC 20555

ONSITE

50 Pacific Northwest Laboratory

DA Baker (35)

JB Brown

CE Elderkin

DW Dragnich

JM Hales

PC Hayes

RA Peloquin

JA Stott lemyre

RE Wildung

Publishing Coordination (2)

Technical Information (5) 



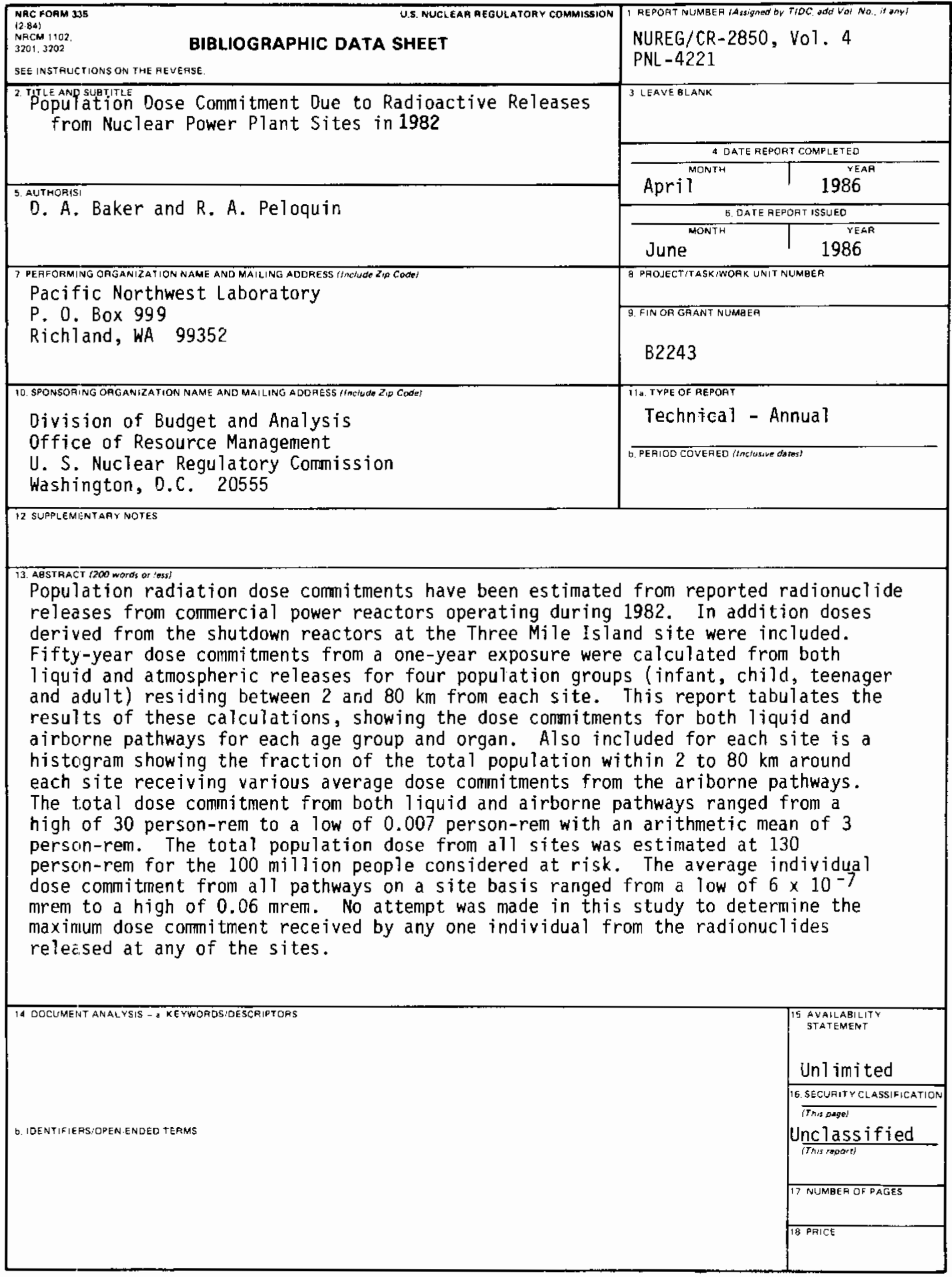


